\title{
STUDIES IN MULTIPARTICLE SCATTERING THEORY
}

\author{
Andrew W. Jackura
}

Submitted to the faculty of the University Graduate School

in partial fulfillment of the requirements

for the degree

Doctor of Philosophy

in the Department of Physics,

Indiana University

May 2019 
Accepted by the Graduate Faculty, Indiana University, in partial fulfillment of the requirements for the degree of Doctor of Philosophy.

\section{Doctoral Committee}

Adam Szczepaniak, Ph.D. (Chair)

Matthew Shepherd, Ph.D.

Jinfeng Liao, Ph.D.

Emilie Passemar, Ph.D.

April 25, 2019 
Copyright (C) 2019

Andrew W. Jackura 


\section{ACKNOWLEDGMENTS}

I must first thank my advisor, Adam Szczepaniak. I started working with Adam my second month in graduate school, where I approached him and asked to work with him on strong interactions. After our first meeting, he had me reading five or six books on strong interacting physics, beginning my journey into this fascinating world. I am sure he has had many frustrating moments as I struggled to understand some problem during my research, where he could have completed it quickly alone. However, these struggles were necessary for me to learn how to tackle problems, and become a researcher. He instilled in me the necessary skills to become a good researcher, and I hope I can continue the trend throughout my career. I cannot have reached this point without your guidance, thank you Adam.

I thank the members of JPAC who have become a big part of my life over the past few years. Special thanks to Vincent Mathieu and Alessandro Pilloni for the many hours we spent having interesting discussions, and to Mikhail Mikhasensko. Misha and I both started our studies at the same time, and being young students we often shared our personal frustrations with each other as we tried to understand our work. We have had numerous (pleasant) arguments about many topics in physics. I am happy to call you a friend.

I thank the entire faculty of the Indiana University Department of Physics for their support and instruction throughout my graduate studies. I thank the friends I have made during my years at IU, especially Blake, Braden, Matthew, Nathan, and Navin.

I could not be here without the support of my family. Thank you for the years of support and encouragement as I pursued this endeavor.

Finally, this work could not have been done without the love and support of my partner, Allison. I love you, and cannot wait for the next chapter of our lives. 


\section{PREFACE}

This work was conducted in the Nuclear Theory Center at Indiana University - Bloomington, and as part of the Joint Physics Analysis Center (JPAC). Portions of this work are largely adapted from Refs. [1-3] and [4]. Specifically, chapter 3 is adapted from Refs. [2] and [3], while and chapters 4 and

5 are taken from [4]. Chapter 5 also contains some currently unpublished work. A small portion of Chapter 3 is adapted from [5], which concerns extensions of the work presented in Ref. [2]. 
Andrew W. Jackura

\section{STUDIES IN MULTIPARTICLE SCATTERING THEORY}

Recent advances in theory and experiment have renewed efforts in the phenomenological understanding of scattering for hadronic systems. Calculations in lattice QCD have shown that resonances can be extracted from the finite volume spectrum. These observables require analytic models to continue the amplitude to the complex energy plane to determine the infinite volume hadron spectrum. The forefront of this research is determining scattering observables for three particles from the finite volume spectra. We present an analytic representation for the elastic three-body scattering amplitude, which can be effectively used in lattice QCD calculations of amplitudes. Aspects of its analytic properties are discussed, specifically the one-particle exchange effects and triangle singularities, which are possible explanations for the many observed exotic hadrons.

Adam Szczepaniak, Ph.D. (Chair)

Matthew Shepherd, Ph.D.

Jinfeng Liao, Ph.D.

Emilie Passemar, Ph.D. 


\section{CONTENTS}

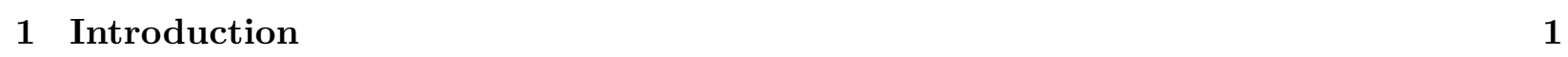

1.1 Strong Interactions and $\mathrm{QCD} \ldots \ldots \ldots \ldots \ldots \ldots$

1.2 The Quark Model . . . . . . . . . . . . . . . . . . . . . . 4

1.3 Three Particle Scattering $\ldots \ldots \ldots \ldots \ldots \ldots$

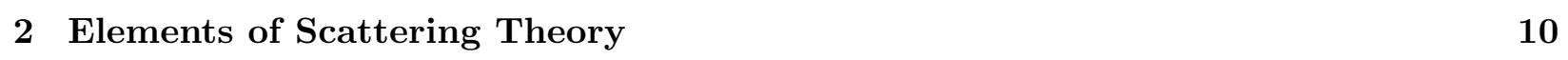

2.1 Unitarity and the S-Matrix $\ldots \ldots \ldots \ldots \ldots \ldots$

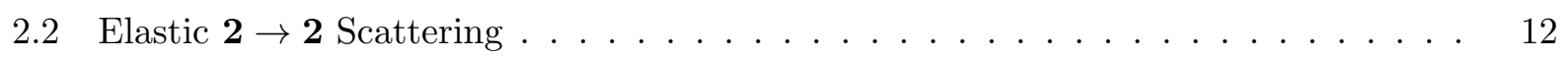

2.3 Including Analyticity $\ldots \ldots \ldots \ldots \ldots \ldots \ldots$

2.4 Resonances . . . . . . . . . . . . . . . . . . . . . . . . . . 18

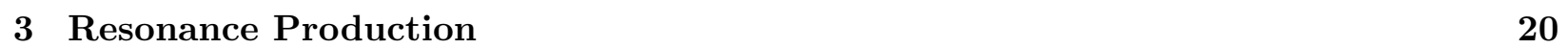

$3.1 \quad \eta \pi$ Resonance Production $\ldots \ldots \ldots \ldots \ldots \ldots \ldots \ldots$

3.1 .1 Production Model . . . . . . . . . . . . . . . . . . . . . . . . 25

3.1 .2 Final State Interactions . . . . . . . . . . . . . . . . . . . . 28

$3.1 .3 \quad$ Analysis of Tensor Resonances . . . . . . . . . . . . . . . . . . 30

3.2 Exotica in $\eta \pi-$ The $\pi_{1}$ Hybrid Candidate $\ldots \ldots \ldots \ldots$. . . . . . . . 42

3.3 Summary $\ldots \ldots \ldots \ldots \ldots \ldots \ldots \ldots$

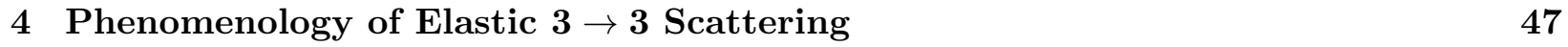

$4.1 \quad$ Kinematics, Invariants, \& Amplitudes $\ldots \ldots \ldots$. . . . . . . . . . . . . . 47

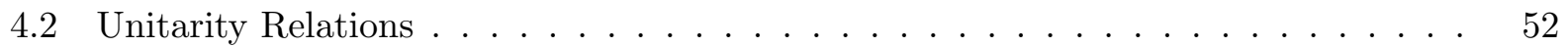

4.3 The Isobar Representation $\ldots \ldots \ldots \ldots$

$4.3 .1 \quad$ PWIS Unitarity Relations $\ldots \ldots \ldots \ldots$. . . . . . . . . . . . 59 
\begin{tabular}{|lll}
\hline 5 & The B-Matrix Representation & 63
\end{tabular}

5.1 Description of the $B$-Matrix Representation $\ldots \ldots \ldots \ldots$. . . . . . . . . 64

$5.2 \quad$ Aspects of Analytic Properties $\ldots \ldots \ldots$

$5.2 .1 \quad$ One Particle Exchange $\ldots \ldots \ldots \ldots$. . . . . . . . . . . . . . 69

5.2 .2 Triangle Diagrams $\ldots \ldots \ldots \ldots \ldots \ldots$

$5.2 .3 \quad$ Removal of Unphysical Singularities $\ldots \ldots \ldots$. . . . . . . . . . . 83

6 Equivalence of Three-Body Formalisms $\quad 85$

6.1 Connecting the $R$-Matrix to $\mathcal{K}_{\mathrm{df}} \ldots \ldots \ldots \ldots \ldots \ldots$

\begin{tabular}{llr|}
\hline 7 & Conclusion & 89
\end{tabular}

\begin{tabular}{lr}
\hline Bibliography & 90
\end{tabular}

\begin{tabular}{|ll}
\hline A Kinematics for $3 \rightarrow 3$ Reactions & 101
\end{tabular}

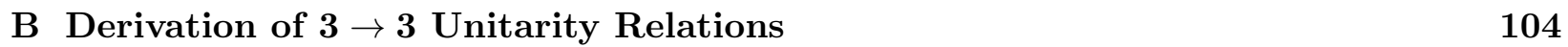

\begin{tabular}{|l|l}
\hline C Derivation of PWIS Unitarity Relations & 107
\end{tabular}

\begin{tabular}{|l|l}
\hline D The $B$-matrix and Unitarity & 115
\end{tabular}

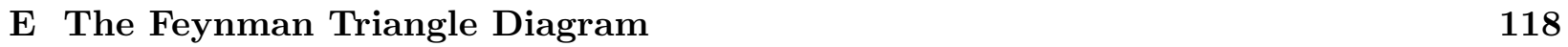

\section{Curriculum Vitae}




\section{Chapter 1}

\section{INTRODUCTION}

More than $99 \%$ of the visible mass in the universe is due to nuclei and their interactions. Unlike electromagnetic phenomena, which has long-range effects, nuclear systems are associated with short-distance physics on the scale of $\sim 10^{-15} \mathrm{~m}$. Nuclear processes are broken down into two categories, weak and strong nuclear phenomena. Weak interactions, which describes for instance the neutron $\beta$-decay $n \rightarrow p+e^{-}+\bar{\nu}_{e}$, are characterized by small cross sections $\left(\sim 10^{-35} \mathrm{~m}^{2}\right)$, and lifetimes of the order of $\gtrsim 10^{-10} \mathrm{~s}$. Conversely, strong interactions have much larger cross sections $\left(\sim 10^{-29} \mathrm{~m}^{2}\right)$ and shorter lifetimes $\left(\sim 10^{-24} \mathrm{~s}\right)$. They are responsible for the binding of nucleons inside nuclei, and for the generation of the long list of short-lived states that populate the PDG [6]. At a more fundamental level, strong interactions generate $99 \%$ of the mass of the nucleons, with the rest being due to the interaction with the electromagnetic and the Higgs fields. States subjected to strong interactions are called hadrons.

\subsection{STRONG INTERACTIONS AND QCD}

Quantum Chromodynamics (QCD) is universally acknowledged as the theory describing strong interactions, and reduces any hadron phenomena to the interactions of quarks mediated by massless gluons $[7]$. These fundamental degrees of freedom are never observed freely (confinement), but can only appear in neutral composite structures, which are identified with the hadrons. The nuclear force between nucleons is then analogous to the Van der Waals forces in molecules. Hadrons are 

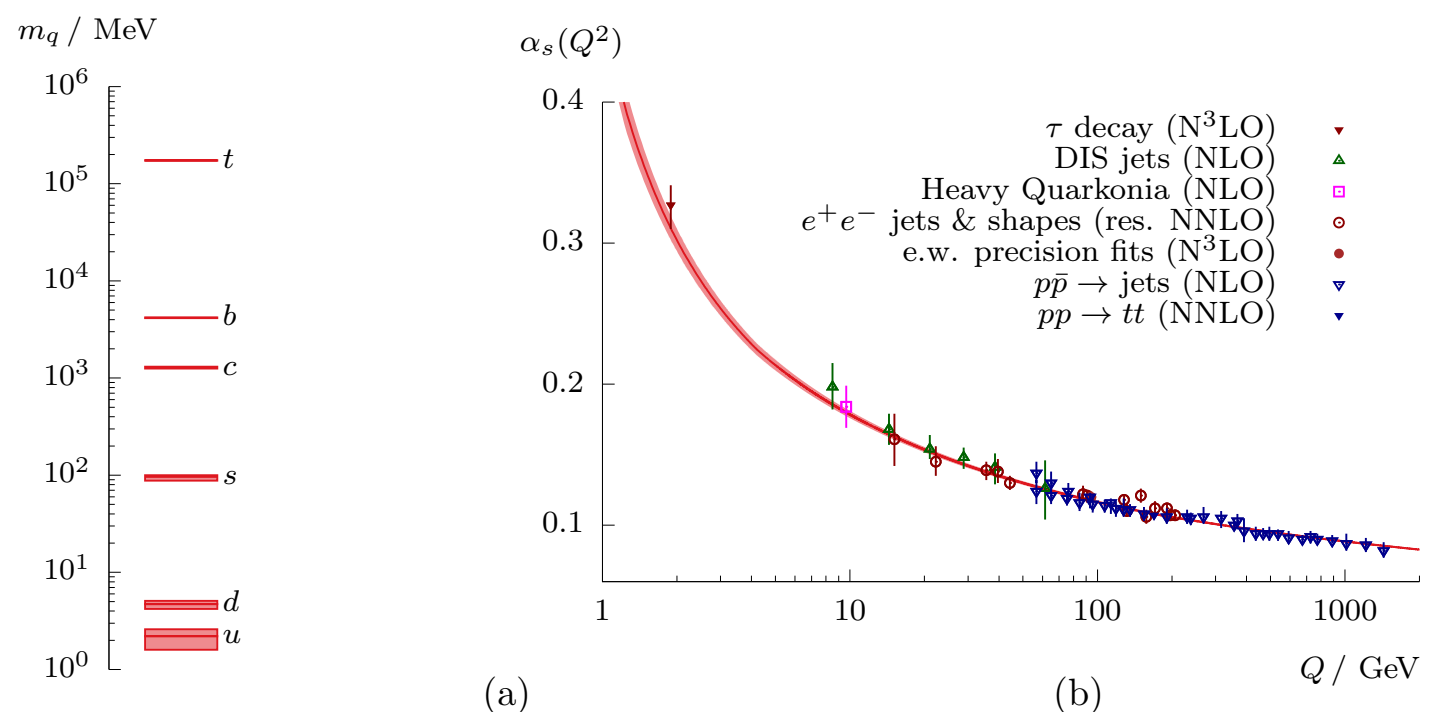

Figure 1.1: The quark mass spectrum (a) and the running of the QCD coupling (b). The quark masses and given are computed in the $\overline{\mathrm{MS}}$ scheme, as in the running coupling. The coupling is such that $\alpha_{s}\left(M_{z}^{2}\right)=0.1181 \pm 0.0011$. The coupling is computed in perturbation theory. Figure adapted from Ref. [6].

the emergent states of quark and gluon interactions, and come in two known classes: mesons and baryons. Mesons are composed of valence quark-antiquark pairs surrounded by a sea of quarks and gluons, and baryons are made up of three valence quarks in the quark-gluon sea. QCD is a relativistic quantum gauge field theory, which is invariant under the non-abelian gauge group $\mathrm{SU}(3)_{c}$, where $c$ means color. QCD can be compactly written with the Lagrangian density

$$
\mathcal{L}_{\mathrm{QCD}}=\frac{i}{2} \sum_{f} \overline{\boldsymbol{\psi}}_{f} \not \mathbf{D} \boldsymbol{\psi}_{f}+\text { h.c. }-\sum_{f} \overline{\boldsymbol{\psi}}_{f} m_{f} \boldsymbol{\psi}_{f}-\frac{1}{2} \operatorname{tr}\left[\mathbf{G}_{\mu \nu} \mathbf{G}^{\mu \nu}\right]
$$

The field $\boldsymbol{\psi}_{f}$ is the quark field for a flavor $f$, and $\mathbf{G}^{\mu \nu}$ is the gluon field strength. Hiding inside this deceptively simple theory is all the physics of nuclear phenomena. Figure 1.1 shows the quark mass spectrum and the running QCD coupling. Indeed in the infrared region, the coupling grows, and perturbative analyses become unreliable.

Recently, tremendous progress has been made in computing hadron properties directly from QCD using lattice QCD. Lattice QCD is a non-perturbative approach to compute observables 


\section{$M / \mathrm{GeV}$}

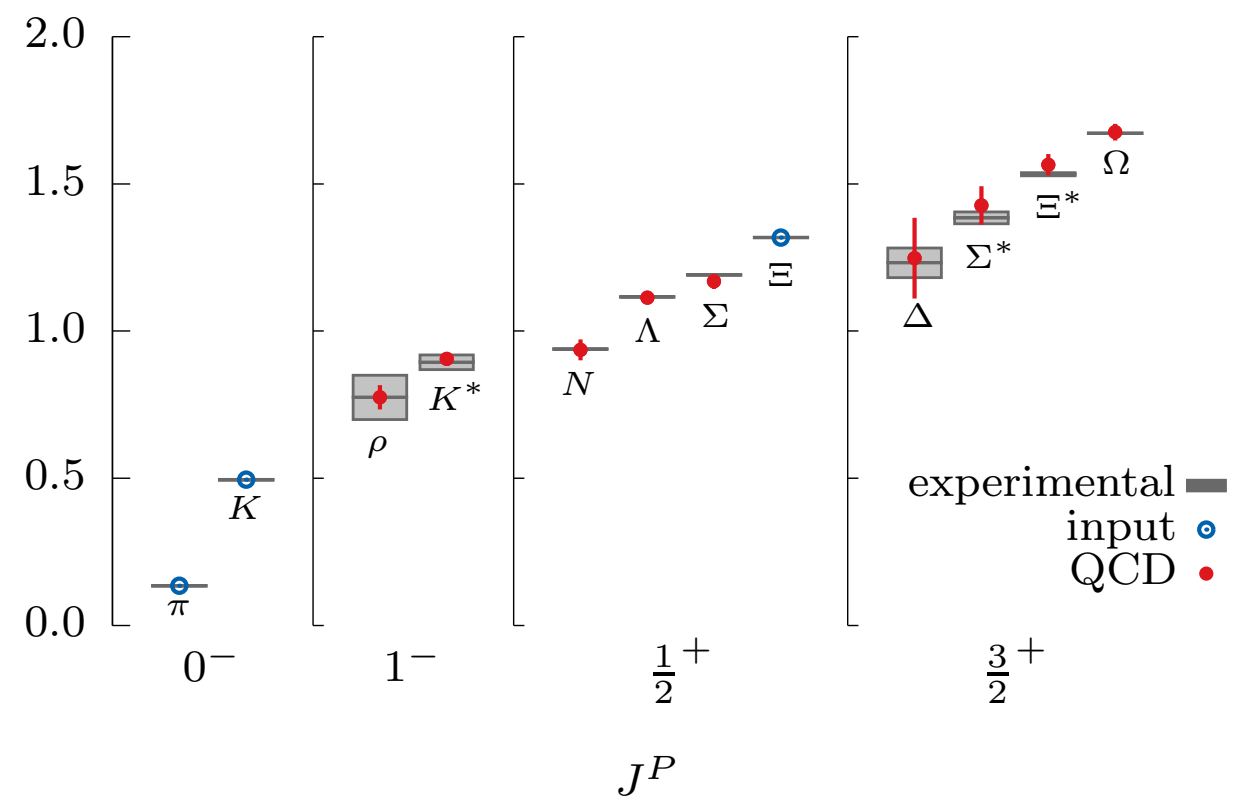

Figure 1.2: The light hadron spectrum computed from lattice QCD. Figure adapted from Ref. |8|

directly from QCD in a discretized, Euclidean spacetime in a finite volume. It is systematically improvable, since one can decrease the discretization size and increase the volume, approaching the continuum limit. Figure 1.2 shows the ground state spectrum computed in lattice QCD from Ref. 8. There exists a non-perturbative methodology that connects the finite volume spectra to infinite volume scattering observables. The essential idea is to compute finite volume corrections to QFT correlation functions, and relate these corrections to scattering quantities. For example, consider the four-point finite volume correlation function in momentum space, $C_{L}(E, \mathbf{P} ; L)$, for the creation and annihilation of two hadron interpolating operator at separated spacetime events in some cube of size $L$. The finite volume correlation function is related to the infinite volume correlation function

$$
C_{L}(E, \mathbf{P} ; L)=C_{\infty}(E, \mathbf{P})+V(E, \mathbf{P} ; L)+\mathcal{O}\left(e^{-m L}\right)
$$

where $V$ is a function containing power law, i.e. $\mathcal{O}\left(L^{n}\right)$, corrections, and $\mathcal{O}\left(e^{-m L}\right)$ are exponentially suppressed corrections, which for suitable $m L>1$ can be ignored. We make the observations that 
$C_{L}$ must contain only poles in $E$, whereas $C_{\infty}$ must contain only cuts (ignoring bound states). Thus, the finite volume correction must have poles at the finite volume energies. This leads to the colloquially called Lüscher quantization condition $[9]$.

The Lüscher quantization condition related the $\mathbf{2} \rightarrow \mathbf{2}$ scattering amplitude to the eigenenergies of the system in the box via

$$
\operatorname{det}\left[F\left(E_{n}, \mathbf{P} ; L\right)+\mathcal{M}\left(E_{n}, \mathbf{P}\right)\right]=0
$$

where $F$ is a matrix in angular momentum space, which characterizes the finite volume effects of two particle propagation, $\mathcal{M}$ the partial wave scattering amplitude, which is a diagonal matrix in angular momentum. The determinant is over all angular momenta. Since the functions $F\left(E_{n}, \mathbf{P} ; L\right)$ are known geometric functions, the condition allows one to compute the scattering amplitude given a two-particle eigenenergy, in a total momentum $\mathbf{P}$, and in a cube of size $L$. The Lüscher quantization condition can be interpreted as a statement of unitarity, as $\operatorname{Im} F\left(E_{n}, \mathbf{P} ; L\right)=-\operatorname{Im} \mathcal{M}\left(E_{n}, \mathbf{P}\right)$. Thus, the amplitude must have the correct structure required by $S$-matrix principles, which are discussed in Chapter 2.

\subsection{THE QUARK MODEL}

The constituent quark model characterizes the hadrons as bound states of constituent quarks $q$ : $q \bar{q}$ for mesons and $q q q$ for baryons. Although similarly named, constituent quarks are not the fundamental quarks of $\mathrm{QCD}$, but are the effective degrees of freedom used to classify the valence structure of the hadrons. Constituent quarks do, however, have most of the same symmetries as the fundamental quarks and come in the same six flavors. The quark model was proposed by Gell-Mann [10] and Zweig [11] in 60s to understanding the hundreds of new particles discovered during he 50s-60s. The hadrons can be classified into $\mathrm{SU}\left(N_{f}\right)_{F}$ multiplets, where $F$ indicates flavor and $N_{f}$ indicates the number of flavors.

Focussing on the mesons, we wish to construct various states arising from $q \bar{q}$ pairs. In the $q \bar{q}$ 

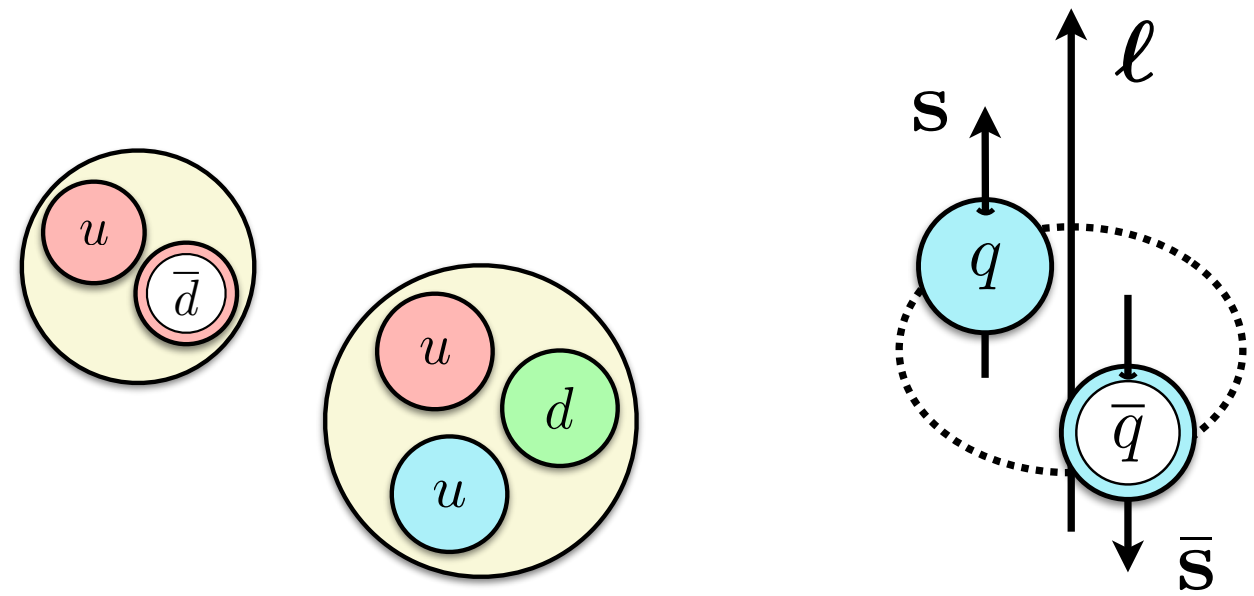

Figure 1.3: Pictoral representation of mesons and baryons in the constituent quark model. The red, green, and blue colors represent the color charge of the hadron, and the white circle with the red outline indicates anti-red to make the meson colorless.

rest frame, the system can couple to a total intrinsic spin $s=\frac{1}{2} \otimes \frac{1}{2}=0$ or 1 , and can have an orbital angular momentum $\ell=0,1,2, \ldots$ which couples to a total angular momentum $\mathbf{J}=\boldsymbol{\ell}+\mathbf{s}$. We consider an effective interaction potential between the quark-antiquark, and assume spherical symmetry with a radial confinement, e.g. the Cornell potential $V(r)=C / r+\sigma r$. This produces a series of states with increasing mass which can be labeled by a radial quantum number $n$. Therefore, we can write the $n^{\text {th }}$-radial excited meson state as

$$
\begin{aligned}
\left|n^{2 s+1} \ell_{J} m_{J}\right\rangle_{q \bar{q}}=\sum_{m_{\ell}, m_{s}}\left\langle\ell m_{\ell} ; s m_{s} \mid J m_{J}\right\rangle \sum_{\sigma, \bar{\sigma}}\left\langle\frac{1}{2} \sigma ; \frac{1}{2} \bar{\sigma} \mid s m_{s}\right\rangle \\
\quad \times \int \frac{\mathrm{d}^{3} \mathbf{p}^{\star}}{(2 \pi)^{3}} \varphi_{n, \ell}\left(p^{\star}\right) Y_{\ell m_{\ell}}\left(\hat{\mathbf{p}}^{\star}\right)\left|q_{\sigma}\left(\mathbf{p}^{\star}\right) \bar{q}_{\bar{\sigma}}\left(-\mathbf{p}^{\star}\right)\right\rangle,
\end{aligned}
$$

where $\mathbf{p}^{\star}$ is the relative momentum of the quark $q$ is the rest frame of the $q \bar{q}$ pair, with magnitude $p^{\star}=\left|\mathbf{p}^{\star}\right|, \sigma(\bar{\sigma})= \pm \frac{1}{2}$ is the intrinsic spin projections of the quark (antiquark), $m_{s}, m_{\ell}$, and $m_{J}$ are the intrinsic spin, orbital, and total angular momentum projections, respectively, $\varphi_{n, \ell}\left(p^{\star}\right)$ is the radial momentum-space wave function, and $Y_{\ell m_{\ell}}\left(\hat{\mathbf{p}}^{\star}\right)$ are the spherical harmonics characterizing the angular dependence. A meson state in-flight with a total momentum $\mathbf{P}$ can subsequently be found by boosting Eq. 1.4 with a standard Lorentz boost. 
The strong interaction preserves parity and charge conjugation symmetry, therefore we can describe our meson state by these two quantum numbers. Acting the $\mathcal{P}$ and $\mathcal{C}$ operators on the state Eq. (1.4) gives the eigenvalues

$$
P=(-1)^{\ell+1}, \quad C=(-1)^{\ell+s}
$$

Recalling that antifermions have opposite intrinsic parity of fermions, the total intrinsic parity is always -1 . Then, from interchanging $\mathbf{p}^{\star} \rightarrow-\mathbf{p}^{\star}$ on the state, we find and additional factor $(-1)^{\ell}$ from the angular dependence, $Y_{\ell m_{\ell}}\left(\hat{\mathbf{p}}^{\star}\right) \rightarrow Y_{\ell m_{\ell}}\left(-\hat{\mathbf{p}}^{\star}\right)=(-1)^{\ell} Y_{\ell m_{\ell}}\left(\hat{\mathbf{p}}^{\star}\right)$. Charge conjugation interchanges $q \leftrightarrow \bar{q}$, which gives factors -1 from fermion interchange, $(-1)^{\ell}$ from the angular component, and $(-1)^{s+1}$ from the spin wavefunction, $\left\langle\frac{1}{2} \bar{\sigma} ; \frac{1}{2} \sigma \mid s m_{s}\right\rangle=(-1)^{s+1}\left\langle\frac{1}{2} \sigma ; \frac{1}{2} \bar{\sigma} \mid s m_{s}\right\rangle$.

Table 1.1 shows the possible $J^{P C}$ and ${ }^{2 s+1} \ell_{J}$ quantum numbers for $q \bar{q}$-mesons in the constituent quark model. Notably absent are the quantum numbers $J^{P C}=0^{--}, 0^{+-}, 1^{-+}, 2^{+-}, \ldots$, which are known as spin-exotic. These spin-exotic mesons are thus forbidden in the constituent quark model, however QCD does not forbid such states as, for example, gluons can play an active role in the binding of fundamental quarks. Spin-exotic hadrons can, however, in principle exists within QCD. Other exotics exists, such as tetraquark or pentaquark states 12. This 'new' exotic hadron spectroscopy began with the discovery of the $\chi_{c 1}(3872)$, also known as the $X(3872)$, by the Belle collaboration in 2003 13. First observed in $B^{ \pm} \rightarrow K^{ \pm} \pi^{+} \pi^{-} J / \psi$ as a narrow peak in the invariant mass of the $\pi^{+} \pi^{-} J / \psi$, this state has properties different from conventional quarkonium states, and is a candidate for an exotic hadron. The $X(3872)$ was determined to have quantum numbers $I^{G}\left(J^{P C}\right)=0^{+}\left(1^{++}\right) 14$. Following the discovery of the $X(3872)$, many other exotic candidates were observed in the heavy quarkonium sector, which are collectively called $X Y Z$ states. Specific examples include the $Z_{c}(3900)$, discovered by Belle and BESII in 2013. 


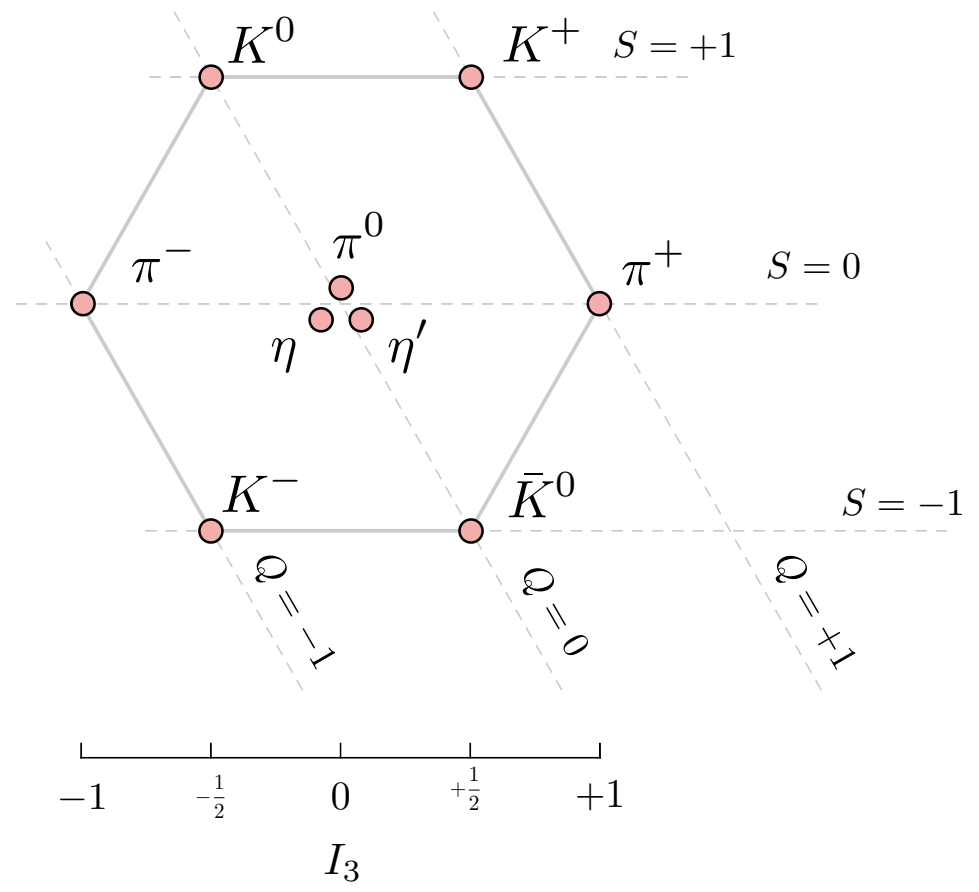

Figure 1.4: The quark model pseudoscalar $\left(J^{P C}=0^{-+}\right)$nonet. The horizontal axis shows the strong isospin projection $I_{3}$. Shown are the charge $Q$ and strangeness $S$ quantum numbers. The quark content of the mesons is $\pi^{+}=u \bar{d}, \pi^{0}=(u \bar{u}-d \bar{d}) / \sqrt{2}, \pi^{-}=d \bar{u}, K^{+}=u \bar{s}, K^{0}=d \bar{s}, \bar{K}^{0}=s \bar{u}$, $K^{-}=s \bar{d}$. The isoscalar $\eta$ and $\eta^{\prime}$ mesons are mixtures of the $\mathrm{SU}(3)_{F}$ states $\eta_{1}=(u \bar{u}+d \bar{d}+s \bar{s}) / \sqrt{3}$ and $\eta_{8}=(u \bar{u}+d \bar{d}-2 s \bar{s}) / \sqrt{6}$, with the mixing $\eta=\eta_{8} \cos \theta_{P}-\eta_{1} \sin \theta_{P}$ and $\eta^{\prime}=\eta_{8} \sin \theta_{P}+\eta_{1} \cos \theta_{P}$, with a pseudoscalar mixing angle of approximately $\theta_{P} \approx-11.5^{\circ}$. 
Table 1.1: Allowed mesonic quantum numbers in the quark model. The total intrinsic spin of the $q \bar{q}$ system is $s=0$ or 1 , and the total angular momentum is restricted by $|\ell-s| \leq J \leq|\ell+s|$, were $\ell$ is the orbital angular momentum. Shown is up through $\ell=3$ ( $F$-wave). The parity of the meson is $P=(-1)^{\ell+1}$, and the $C$-parity is $C=(-1)^{\ell+s}$. Shown are the $J^{P C}$ quantum numbers, and the spectroscopic notation ${ }^{2 s+1} \ell_{J}$.

\begin{tabular}{ccccccc}
$s$ & $\ell$ & $J$ & $P$ & $C$ & $J^{P C}$ & ${ }^{2 s+1} \ell_{J}$ \\
\hline \hline 0 & 0 & 0 & - & + & $0^{-+}$ & ${ }^{1} S_{0}$ \\
0 & 1 & 1 & + & - & $1^{+-}$ & ${ }^{1} P_{1}$ \\
0 & 2 & 2 & - & + & $2^{-+}$ & ${ }^{1} D_{2}$ \\
0 & 3 & 3 & + & - & $3^{+-}$ & ${ }^{1} F_{3}$ \\
\hline 1 & 0 & 1 & - & - & $1^{--}$ & ${ }^{3} S_{1}$ \\
1 & 1 & $0,1,2$ & + & + & $0^{++}, 1^{++}, 2^{++}$ & ${ }^{3} P_{0},{ }^{3} P_{1},{ }^{3} P_{2}$ \\
1 & 2 & $1,2,3$ & - & - & $1^{--}, 2^{--}, 3^{--}$ & ${ }^{3} D_{1},{ }^{3} D_{2},{ }^{3} D_{3}$ \\
1 & 3 & $2,3,4$ & + & + & $2^{++}, 3^{++}, 4^{++}$ & ${ }^{3} F_{2},{ }^{3} F_{3},{ }^{3} F_{4}$ \\
\hline
\end{tabular}

\subsection{THREE PARTICLE SCATTERING}

Modern high-energy experiments are accumulating high quality data on three-hadron final states, that are expected to be the main decay channels of several poorly known or missing resonances. These include, for example, the enigmatic $a_{1}, \omega_{2}$, and the exotic $\pi_{1}$ resonances that can be studied in peripheral production at COMPASS, GlueX, and CLAS12 [?,15 20]. In addition to conventional hadrons, many of the exotic $X Y Z$ and pentaquark states observed in the heavy quarkonium sector [12,21,22], are also found in three particle final states.

Many of these newly observed or anticipated states lie close to thresholds of their decay products. For example, the mass difference between the $X(3872)\left[13\right.$ and the $D^{0} \bar{D}^{0} \pi^{0}$ threshold is only $6 \mathrm{MeV}$. The proximity of the three particle threshold together with the possibility of longrange interactions mediated by a single pion exchange can significantly influence the $X(3872)$ line-shape [23] and one needs to carefully analyze the role of pion exchange and whether it is able 
to bind $D^{* 0}$ and $\bar{D}^{0} 24$ 28]. In the light meson sector, the recently observed $a_{1}(1420)$ 16 is yet another candidate for a state not expected in the quark model that can be influenced by meson exchange interactions and thresholds [29,30].

On the theory side, lattice QCD has made substantial progress in extracting the resonance spectrum from simulations of $\mathbf{2} \rightarrow \mathbf{2}$ reactions $[31,39]$, and recently, the formalism for $\mathbf{3} \rightarrow \mathbf{3}$ scattering has been developed 40 50]. Analysis and interpretation of both experimental data and lattice simulations require input in the form of reaction amplitudes that can be analytically continued into the complex energy plane. For example, in partial waves, resonances appear as pole singularities, while particle exchanges lead to logarithmic branch points. Fortunately, reaction amplitudes are constrained by unitarity, which can be used to determine the discontinuities of partial waves in the near threshold region.

The remainder of this thesis is devoted to phenomenological studies of two and three hadron reactions. We use general principals of scattering theory to restrict the form of reaction amplitudes to be used in data analysis. In Chapter 2, we review key results of scattering theory, setting the stage for analyses presented in subsequent chapters. Chapter 3 applies these results to the hadroproduction of $\eta^{(\prime)} \pi$ resonances, specifically the tensor and exotic sector. We then discuss the phenomenology of elastic $\mathbf{3} \rightarrow \mathbf{3}$ scattering in Chapter 4, and Chapter 5 present a representation for the $\mathbf{3} \rightarrow \mathbf{3}$ amplitude which satisfies unitarity, and in Chapter 6 we compare to other formalisms in the literature. Finally, we conclude in Chapter 7, where we discuss potential future studies to understand three particle systems. 


\section{Chapter 2}

\section{ELEMENTS OF SCATTERING THEORY}

Scattering theory is a framework for studying particle reaction without the need of specifying the underlying interaction between particles. The framework is based on restricting the form of scattering amplitudes by imposing fundamental principles on the system. This includes probability conservation, causality, and Poincaré invariance. These principles can be used to construct amplitude models which can be used to fit to experimental or lattice QCD data, and in turn can be analytically continued to extract physics of the reaction. In this chapter, we state some useful results which will be useful in subsequent chapters.

\subsection{UNITARITY AND THE S-MATRIX}

The probability for an initial quantum state transitioning to a final state is

$$
\operatorname{Prob}_{i \rightarrow f}=|\langle f|S| i\rangle|^{2}
$$

where $|i\rangle$ denotes the normalized initial state, $|f\rangle$ is the final state, and $S$ is the scattering matrix operator, or, the S-Matrix. For some theories, e.g. QED, one computes $S$-matrix elements via a perturbative expansion in the coupling strength, e.g. $\alpha$ for QED. The perturbation series can be represented by Feynman diagrams, which are a set of diagrammatical rules for efficient computation of $S$-matrix elements given the theory. In strongly interacting systems, it is observed that the asymptotic states of the theory, quarks, are not the observed asymptotic states, hadrons. The connection between the input and observed states is not fully understood, and ultimately arises 
from the confinement nature of QCD. In the low-energy region, the coupling is known to be relatively large, and perturbative methods breakdown. However, we can work directly with scattering amplitude of hadronic reactions and use general principles of physics to constrain the scattering amplitude.

The primary constraint is unitarity, which is a statement that the total probability for an initial state to transition to any final state must be unity according to conservation of probability,

$$
\sum_{f} \operatorname{Prob}_{i \rightarrow f}=\sum_{f}|\langle f|S| i\rangle|^{2}=1 .
$$

This statement has important consequences on scattering amplitudes. We first observe that the $S$-matrix must be a unitary operator,

$$
S^{\dagger} S=S S^{\dagger}=\mathbb{1}
$$

From the connectedness structure Eq. (2.4), We define the $T$-matrix operator as the piece of the $S$-matrix which contains interactions, and the identity represents where the particles pass through without interacting,

$$
S=\mathbb{1}+i T
$$

The $T$-matrix operator therefore satisfies the relation

$$
T-T^{\dagger}=i T^{\dagger} T=i T T^{\dagger}
$$

which is the general unitarity relation for the $T$-matrix operator. We define scattering amplitudes as matrix elements of the $T$-matrix, thus the unitarity relation an amplitude for an $\mathbf{n} \rightarrow \mathbf{n}^{\prime}$ system is

$$
2 \operatorname{Im}\left\langle\left\{\mathbf{p}^{\prime}\right\}_{\mathbf{n}^{\prime}}|T|\{\mathbf{p}\}_{\mathbf{n}}\right\rangle=\sum \int \prod_{j} \frac{\mathrm{d}^{3} \mathbf{k}_{j}}{(2 \pi)^{3} 2 \omega_{j}\left(\mathbf{k}_{j}\right)}\left\langle\left\{\mathbf{p}^{\prime}\right\}_{\mathbf{n}^{\prime}}\left|T^{\dagger}\right|\{\mathbf{k}\}_{\mathbf{m}}\right\rangle\left\langle\{\mathbf{k}\}_{\mathbf{m}}|T|\{\mathbf{p}\}_{\mathbf{n}}\right\rangle .
$$

The states describe a general multiparticle state. A single on-shell scalar particle of mass $m$ and four-momentum $p=(\omega(\mathbf{p}), \mathbf{p})$, where $\omega(\mathbf{p})=\sqrt{m^{2}+|\mathbf{p}|^{2}}$ is the energy of the particle. The particle can be represented by the state $|\mathbf{p}\rangle$, which we choose the relativistic normalization

$$
\left\langle\mathbf{p}^{\prime} \mid \mathbf{p}\right\rangle=(2 \pi)^{3} 2 \omega(\mathbf{p}) \delta^{(3)}\left(\mathbf{p}^{\prime}-\mathbf{p}\right) .
$$




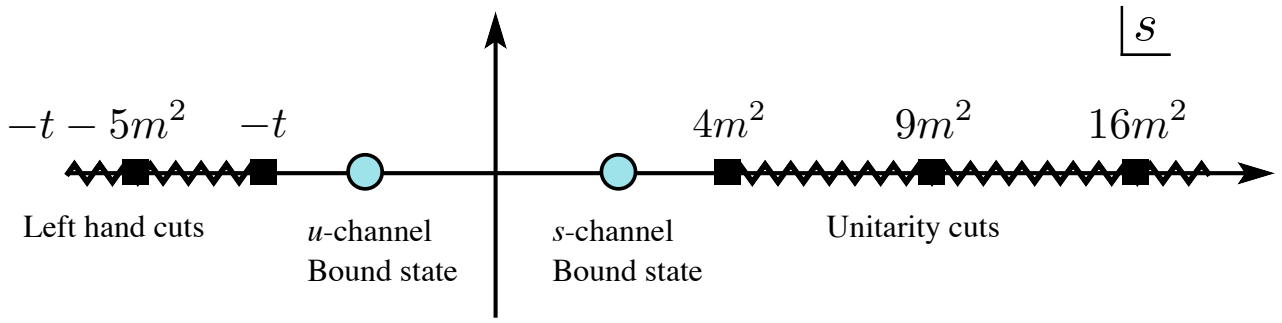

Figure 2.1: Analytic structure of a $\mathbf{2} \rightarrow \mathbf{2}$ amplitude in the complex $s$-plane at fixed $t$. From the kinematic relation $s+t+u=4 m^{2}$, the $u$-channel thresholds $(2 m)^{2},(3 m)^{2}, \ldots$ manifest themselves as $s$-channel left hand cuts.

Multiparticle states can be constructed via the tensor product, e.g. a two particle state $\left|\mathbf{p}_{1} \mathbf{p}_{2}\right\rangle=$ $\left|\mathbf{p}_{1}\right\rangle \otimes\left|\mathbf{p}_{2}\right\rangle$ for distinguishable particles and $\left|\mathbf{p}_{1} \mathbf{p}_{2}\right\rangle=\left(\left|\mathbf{p}_{1}\right\rangle \otimes\left|\mathbf{p}_{2}\right\rangle+\left|\mathbf{p}_{2}\right\rangle \otimes\left|\mathbf{p}_{1}\right\rangle\right) / \sqrt{2}$. Unitarity fixes the imaginary part of the scattering amplitude, and shows that for each new intermediate state, a branch cut occurs starting at the threshold for the state. For a two-particle scattering system, this is shown pictorially in Fig 2.1.

\subsection{ELASTIC $2 \rightarrow 2$ SCATTERING}

As an application of scattering theory, let us consider the elastic scattering of two hadrons. For simplicity, we assume that both hadrons are pseudoscalar particles with equal mass $m$, e.g. elastic $\pi \pi$ scattering. We also assume that we are interested in an energy window $2 m \leq E^{\star}<E_{\text {inel }}^{\star}$, where $E_{\text {inel }}^{\star}$ is the first inelastic threshold. The elastic $\mathbf{2} \rightarrow \mathbf{2}$ scattering amplitude is defined as

$$
\left\langle\mathbf{q}^{\prime \star}|T| \mathbf{q}^{\star}\right\rangle=(2 \pi)^{4} \delta^{(4)}\left(P^{\prime}-P\right) \mathcal{M}\left(\mathbf{q}^{\prime \star}, \mathbf{q}^{\star}\right),
$$

where we defined the amplitude in terms of its CMF variables. Here, $\mathbf{q}^{\star}$ and $\mathbf{q}^{\prime \star}$ are the initial and final CMF relative momenta, with magnitude

$$
q^{\star}=\left|\mathbf{q}^{\star}\right|=\left|\mathbf{q}^{\prime \star}\right|=\frac{1}{2} \sqrt{s-4 m^{2}} .
$$




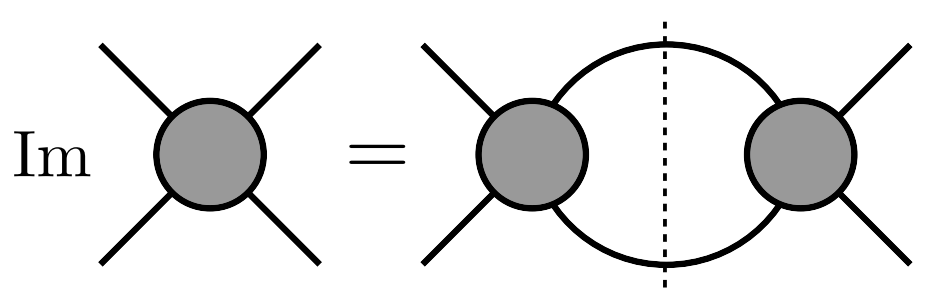

Figure 2.2: Diagrammatic representation of the elastic $\mathbf{2} \rightarrow \mathbf{2}$ unitarity condition, Eq. (2.10). The grey blob represents the $\mathbf{2} \rightarrow \mathbf{2}$ amplitude $\mathcal{M}$ while the dotted vertical line represents the cut putting the intermediate particles on-shell. Amplitudes to the left of the cut are complex conjugated.

The scattering angle between the initial and final state, $\Theta^{\star}$, is defined via $\cos \Theta^{\star}=\hat{\mathbf{q}}^{\prime \star} \cdot \hat{\mathbf{q}}^{\star}$. The elastic $\mathbf{2} \rightarrow \mathbf{2}$ unitarity relation

$$
\operatorname{Im} \mathcal{M}\left(\mathbf{q}^{\prime \star}, \mathbf{q}^{\star}\right)=\rho(s) \int \frac{\mathrm{d} \hat{\mathbf{k}}^{\star}}{4 \pi} \mathcal{M}^{*}\left(\mathbf{q}^{\prime \star}, \mathbf{k}^{\star}\right) \mathcal{M}\left(\mathbf{k}^{\star}, \mathbf{q}^{\star}\right) \Theta(\sqrt{s}-2 m)
$$

where $\rho(s)$ is defined as the two body phase space,

$$
\rho(s)=\frac{1}{16 \pi} \frac{q^{\star}}{\sqrt{s}}=\frac{1}{32 \pi} \sqrt{1-\frac{4 m^{2}}{s}} .
$$

Equation 2.10 can be represented diagrammatically, as shown in Fig. 2.2.

We can simplify the unitarity relation by introducing a basis that diagonalizes the equation. The two particle state is a reducible representation of the orthochronus Poincaré group. We can write the two particle state in an irreducible representation by projecting the orientation of the state into definite angular momentum quantum numbers,

$$
\left|q^{\star}, \hat{\mathbf{q}}^{\star}\right\rangle=\sqrt{4 \pi} \sum_{\ell=0}^{\infty} \sum_{m_{\ell}=-\ell}^{\ell} Y_{\ell m_{\ell}}^{*}\left(\hat{\mathbf{q}}^{\star}\right)\left|q^{\star}, \ell m_{\ell}\right\rangle
$$

where $\ell$ is the total angular momentum of the system, $m_{\ell}$ is the projection along some predefined axis, and $Y_{\ell m_{\ell}}\left(\hat{\mathbf{q}}^{\star}\right)=\left\langle\hat{\mathbf{q}}^{\star} \mid \ell m_{\ell}\right\rangle$ is a spherical harmonic which characterizes the rotational dependence of the state. The factor $\sqrt{4 \pi}$ is for convenience. Effectively, $\left|q^{\star}, \ell m_{\ell}\right\rangle$ represents a state of invariant mass $\sqrt{s}$ and "spin" $\ell$. Projecting the initial and final state into definite angular momenta 
results in the partial wave expansion (PWE)

$$
\mathcal{M}\left(\mathbf{q}^{\prime \star}, \mathbf{q}^{\star}\right)=4 \pi \sum_{\ell=0}^{\infty} \mathcal{M}_{\ell}(s) \sum_{m_{\ell}=-\ell}^{\ell} Y_{\ell m_{\ell}}\left(\hat{\mathbf{q}}^{\prime \star}\right) Y_{\ell m_{\ell}}^{*}\left(\hat{\mathbf{q}}^{\star}\right),
$$

where $\mathcal{M}_{\ell}$ is the partial wave amplitude, which depends only on the invariant mass of the system. Note that since the total angular momentum is conserved, the partial wave amplitude is diagonal in $\ell$, and independent of $m_{\ell}$ as is easily seen by the Wigner-Eckart theorem,

$$
\mathcal{M}_{\ell^{\prime} m_{\ell}^{\prime} ; \ell m_{\ell}}(s)=\delta_{\ell^{\prime} \ell} \delta_{m_{\ell}^{\prime} m_{\ell}} \mathcal{M}_{\ell}(s)
$$

The PWE separates the rotational dependence of the amplitude into known functions. Recall that the amplitude is crossing symmetric, i.e. the same amplitude describes the physics of the $s^{-}, t^{-}$, and $u$-channel processes. The partial wave amplitude $\mathcal{M}_{\ell}(s)$, however, depends only on $s$ and thus only describes $s$-channel singularities. The $t$-channel dependence is encoded in the angular dependence, which is described by the spherical harmonics which are regular functions. To recover singularities in the crossed channel, e.g. the $t$-channel, one must sum the infinite series. An illustration of recovering singularities from an infinite sum is the geometric series $\sum_{j=0}^{\infty} z^{j}=(1-z)^{-1}$, where a pole singularity exist at $z=1$.

Using the orthonormality properties of the spherical harmonics, the PWE diagonalizes the elastic unitarity relation

$$
\operatorname{Im} \mathcal{M}_{\ell}(s)=\rho(s)\left|\mathcal{M}_{\ell}(s)\right|^{2} \Theta(\sqrt{s}-2 m)
$$

which is an algebraic equation known as the elastic partial wave unitarity relation. The unitarity relation provides a powerful tool in restricting the forms of scattering amplitudes. We would expect to provide one complex number (two real numbers) for each value of $s$. Equation 2.15) however, fixes the imaginary part, implying one real number is needed to determine the amplitude for each $s$. We introduce the phase shift $\delta_{\ell}(s)$ via $\mathcal{M}_{\ell}(s)=\left|\mathcal{M}_{\ell}(s)\right| e^{i \delta_{\ell}(s)}$, and fix the magnitude via Eq. 2.15) to attain the phase shift formula

$$
\mathcal{M}_{\ell}(s)=\frac{1}{\rho(s)} e^{i \delta_{\ell}(s)} \sin \delta_{\ell}(s)
$$


To specify any particular scattering process, one needs to determine the scattering phase shift. An alternative representation of the on-shell partial wave amplitude is the $K$-matrix,

$$
\mathcal{M}_{\ell}(s)=\mathcal{K}_{\ell}(s) \frac{1}{1-i \rho(s) \mathcal{K}_{\ell}(s)}
$$

where $\mathcal{K}_{\ell}$ is a real function in the elastic kinematic region. The $K$-matrix representation shows some main features of the partial wave amplitude. First, the kinematics for on-shell pair production

is encoded in the two-body phase space factor. Second, the form shows that an infinite sum of rescatterings has been taken, which is known as unitarization. Finally, the $K$-matrix representation is independent of any specific process or theory. The $K$-matrix itself contains all the dynamical information for a specific process, e.g. from ChiPT, QCD, etc.. In order to ensure no poles exist on the first Riemann sheet, one can introduce the $K$-matrix parameterization

$$
\mathcal{K}_{\ell}^{-1}(s)=c_{\ell}^{(0)}-c_{\ell}^{(1)} s-\sum_{j} \frac{c_{\ell, j}^{(2)}}{c_{\ell, j}^{(3)}-s},
$$

where the $c_{\ell}$ coefficients are real parameters, all positive definite. The terms in the $j$-sum are called CDD poles [51], and the first two terms combined is called a CDD pole at infinity. Other parameterizations exist which are commonly found in the literature. The $K$-matrix representation is useful for analytical continuation, as will be discussed in Sec. 2.4.

\subsection{INCLUDING ANALYTICITY}

So far, we have only considered unitarity is constructing a representation for the scattering amplitude. We can improve the description by writing a dispersive representation for the amplitude. It is useful to rewrite the unitarity relation in terms of the inverse amplitude

$$
\operatorname{Im} \mathcal{M}_{\ell}^{-1}(s)=-\rho(s) \Theta(\sqrt{s}-2 m)
$$

We can use Cauchy's theorem [?] to then write a dispersive representation for the inverse amplitude,

$$
\mathcal{M}_{\ell}^{-1}(s)=\mathcal{C}_{\ell}^{-1}(s)-\frac{s}{\pi} \int_{4 m^{2}}^{\infty} \mathrm{d} s^{\prime} \frac{\rho\left(s^{\prime}\right)}{s^{\prime}\left(s^{\prime}-s-i \epsilon\right)}
$$


where we have explicitly included the prescription $s+i \epsilon$ to avoid the branch cut. The function $\mathcal{C}_{\ell}$ is a real function which contains the short range dynamics of the system. Note that since $\rho(s) \rightarrow$ constant as $s \rightarrow \infty$, the dispersion relation requires at least one subtraction in order to ensure a UV finite quantity. This is equivalent to choosing a renormalization scheme in perturbative QFT's. Any subtraction constant introduced can be absorbed into the real quantity that defines the specific interaction. The $K$-matrix and $\mathcal{C}$ are related via

$$
\mathcal{K}_{\ell}^{-1}(s)=\mathcal{C}_{\ell}^{-1}(s)-\frac{s}{\pi} \mathcal{P} \int_{4 m^{2}}^{\infty} \mathrm{d} s^{\prime} \frac{\rho\left(s^{\prime}\right)}{s^{\prime}\left(s^{\prime}-s\right)}
$$

We see the difference is a real function, which in undetermined by unitarity but explicitly given from considering its analyticity properties. As indicated, unitarity only determines the imaginary part of the amplitude, but $\mathcal{K}_{\ell}$ allows the flexibility to introduce expected behavior of the amplitude.

Alternatively, knowing that the first sheet amplitudes have both right and left hand cuts, we can write a dispersive representation for the full amplitude as

$$
\mathcal{M}_{\ell}(s)=\frac{1}{\pi} \int_{L} \mathrm{~d} s^{\prime} \frac{\operatorname{Im} \mathcal{M}_{\ell}\left(s^{\prime}\right)}{s^{\prime}-s}+\frac{1}{\pi} \int_{4 m^{2}}^{\infty} \mathrm{d} s^{\prime} \frac{\rho\left(s^{\prime}\right)\left|\mathcal{M}_{\ell}\left(s^{\prime}\right)\right|^{2}}{s^{\prime}-s}
$$

where subtractions can be included as needed. This nonlinear integral equation is difficult to solve, however, we may use the $N / D$ techniques of Ref. [52 to linearize the equations. We write the amplitude as the ratio

$$
\mathcal{M}_{\ell}(s)=\mathcal{N}_{\ell}(s) / \mathcal{D}_{\ell}(s)
$$

where $\mathcal{N}_{\ell}$ contains all the left hand cuts, and $\mathcal{D}_{\ell}$ contains all the right hand cuts. Then, given a specific model for $\mathcal{N}_{\ell}$, i.e. some exchange process, the $\mathcal{D}_{\ell}$ function can be reconstructed via the integral

$$
D_{\ell}(s)=D_{\ell}^{(0)}(s)-\frac{1}{\pi} \int_{s_{\mathrm{th}}}^{\infty} \mathrm{d} s^{\prime} \frac{\rho\left(s^{\prime}\right) N_{\ell}\left(s^{\prime}\right)}{s^{\prime}-s-i \epsilon},
$$

where $D_{\ell}^{(0)}(s)$ is a real function that represents the CDD poles 51 . 


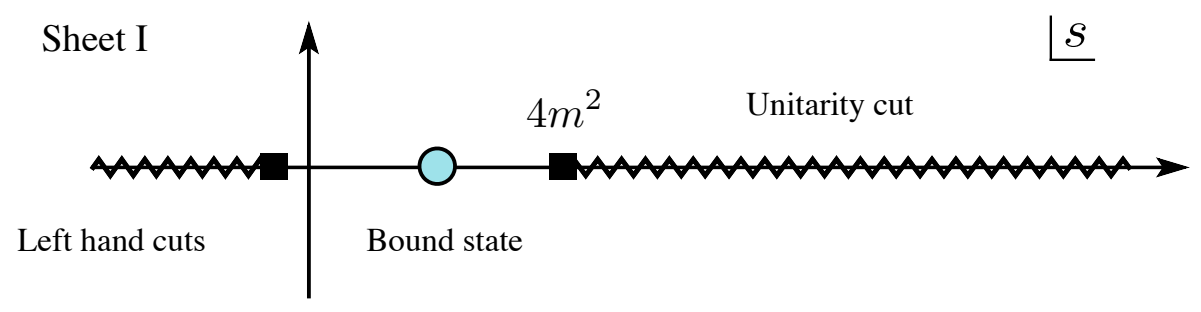

(a)

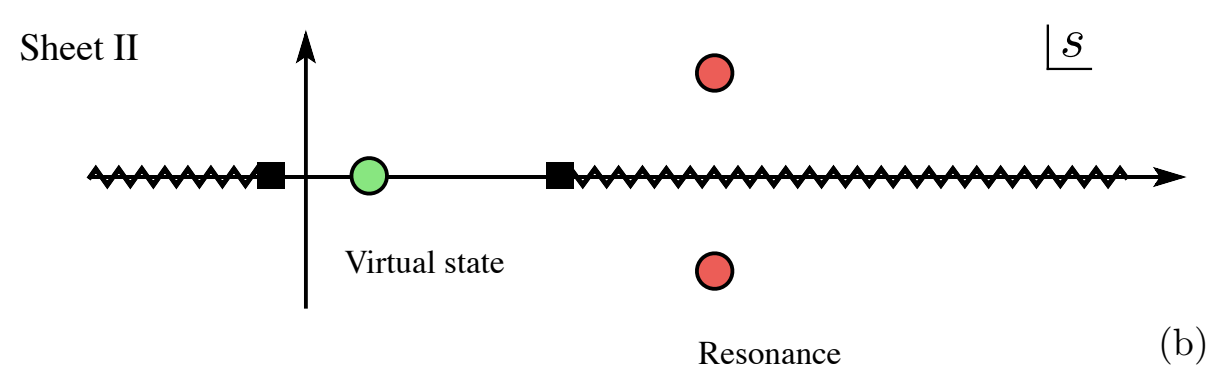

Figure 2.3: Analytic structure of partial wave amplitudes in the Complex s-plane. The first Riemann sheet (a) contains the unitarity cut beginning at the threshold $4 m^{2}$, possible bound state poles, and left hand cuts stemming from exchange processes. The second Riemann sheet (b) contains the unitarity and left hand cuts, as well as potential virtual state and resonance poles. Note that resonance poles come in pairs, however only the one nearest the physical region is contributes dominantly to the real axis lineshape. 


\subsection{RESONANCES}

The partial wave amplitude is an analytic function in the complex $s$-plane except for bound state poles on the real axis, the unitarity cut starting at the branch point $s=4 m^{2}$, and "left-hand cuts" arising from partial wave projections of crossed-channel diagrams 1 . Since resonance poles must have a finite imaginary part, their poles are expected to lie somewhere in the upper or lower complex s-plane. However, as we have seen from the principle of causality, the scattering amplitude cannot have complex poles in the $s$-plane. Thus, resonance poles must lie on the unphysical sheet, close to the physical region. The second sheet amplitude is continuously connected to the first sheet amplitude through the unitarity cut,

$$
\mathcal{M}_{\ell}(s+i \epsilon)=\mathcal{M}_{\ell}^{\mathrm{II}}(s-i \epsilon),
$$

for $s>4 m^{2}$. Using this property, and the unitarity relation can be reexpressed in terms of the difference of the first and second sheet amplitudes,

$$
\mathcal{M}_{\ell}^{\mathrm{II}}(s-i \epsilon)=\mathcal{M}_{\ell}(s-i \epsilon)+\Delta_{s} \mathcal{M}_{\ell}(s)
$$

since unitarity relates the discontinuity across the branch cut, $2 i \operatorname{Im} \mathcal{M}_{\ell}(s)=\mathcal{M}(s+i \epsilon)-\mathcal{M}(s-i \epsilon)$. This yields the continuation of the first sheet amplitude as

$$
\mathcal{M}_{\ell}^{\mathrm{II}}(s)=\mathcal{M}_{\ell}(s) \frac{1}{1+2 i \rho(s) \mathcal{M}_{\ell}(s)}
$$

Since $\mathcal{M}_{\ell}$ has no poles, the only case where a pole can develop is when

$$
1+2 i \rho\left(s_{R}\right) \mathcal{M}_{\ell}\left(s_{R}\right)=0
$$

where $s_{R}$ is the location of the resonance pole. It is convention to define the resonance mass $m_{R}$ and width $\Gamma_{R}$ as

$$
\sqrt{s_{R}}=m_{R}-\frac{i}{2} \Gamma_{R}
$$

\footnotetext{
${ }^{1}$ These cuts in general could be complicated, such as the circular cut arising from projecting the one-particle exchange diagram.
} 
The residue at the pole characterizes the coupling strength, $g_{\ell, R}$, of the resonance to the channel,

$$
\operatorname{Res}\left(\mathcal{M}_{\ell}^{\mathrm{II}} ; s \rightarrow s_{R}\right)=\frac{1}{2 \pi i} \oint_{\gamma} \mathrm{d} s^{\prime} \mathcal{M}_{\ell}^{\mathrm{II}}\left(s^{\prime}\right) \equiv g_{\ell, R}^{2},
$$

where $\gamma$ is a contour in the $s^{\prime}$-plane which encloses only the resonance pole $s_{R}$. A resonance then has the following properties: A mass $m_{R}=\operatorname{Re} \sqrt{s_{R}}$, decay width $\Gamma_{R}=-2 \operatorname{Im} \sqrt{s_{R}}$, a spin $\ell$, a coupling $g_{\ell, R}$, and parity $P=(-1)^{\ell}$. Figure 2.3 shows a cartoon of the various singularities on the first and second Riemann sheet.

We have seen that we can constrain the form of the scattering amplitude into a analytic function in the complex s-plane, which depends on a real function which contains the dynamics of the system. In practical hadronic processes, we do not know $K$, however we can parameterize the function with some simple, flexible form and fit the function to experimental data. Then, the function may be analytically continued using the unitarity relation, and resonance parameters can be determined. 


\section{Chapter 3}

\section{RESONANCE PRODUCTION}

Having discussed key results of scattering theory, we now apply these results to determine properties of hadrons from experimental data. Our focus is on $\eta^{(\prime)} \pi$ resonant hadroproduction, specifically we use the COMPASS dataset to benchmark these methods. In 2014, COMPASS published highstatistics partial wave analyses of the $\pi^{-} p \rightarrow \eta^{(\prime)} \pi^{-} p$ reaction, at $p_{\text {beam }}=191 \mathrm{GeV}[15$. The odd angular-momentum waves have exotic quantum numbers and exhibit structures that may be compatible with a hybrid meson [53]. The even waves show strong signals of non-exotic resonances. In particular, the $D$-wave of $\eta \pi$, with $I^{G}\left(J^{P C}\right)=1^{-}\left(2^{++}\right)$, is dominated by the peak of the $a_{2}(1320)$ and its Breit-Wigner parameters were extracted and presented in Ref. [15]. The $D$-wave also exhibits a hint of the first radial excitation, the $a_{2}^{\prime}(1700)[6]$.

Using the 2014 COMPASS measurement as input, the model is fitted to the results of the mass-independent analysis that was performed in $40 \mathrm{MeV}$ wide bins of the $\eta \pi$ mass. The $a_{2}$ and $a_{2}^{\prime}$ resonance parameters are extracted in the single-channel approximation and the coupled-channel effects are estimated by including the $\rho \pi$ final state. We determine the statistical uncertainties by means of the bootstrap method [?,54 57$]$, and assess the systematic uncertainties in the pole positions by varying model-dependent parameters in the reaction amplitude. We then briefly discuss a natural extension of the model to include $\eta^{\prime} \pi$ in a coupled channel analysis. The exotic $P$-wave channel was investigated, and a determination of a light hybrid meson candidate was determined [5]. 


\section{$3.1 \eta \pi$ RESONANCE PRODUCTION}

We define the $\eta \pi$ production amplitude as

$$
\begin{aligned}
\left\langle\eta\left(p_{1}\right) \pi^{-}\left(p_{2}\right) N\left(p_{d}, \mu^{\prime}\right)\right| & \left|\pi^{-}\left(p_{a}\right) N\left(p_{b}, \mu\right)\right\rangle \\
& =(2 \pi)^{4} \delta^{(4)}\left(P^{\prime}-P\right) \mathcal{A}_{\mu^{\prime} \mu}\left(s, t, m_{\eta \pi}, t_{\eta \pi}, s_{\pi N}\right),
\end{aligned}
$$

where $P=p_{a}+p_{b}$ and $P^{\prime}=p_{1}+p_{2}+p_{d}$ are the initial and final total four-momenta, and the production amplitude $\mathcal{A}_{\mu^{\prime} \mu}\left(s, t, m_{\eta \pi}, t_{\eta \pi}, s_{\pi N}\right)$ depends on the helicities of the initial and final nucleons, and five kinematic variables, which we take to be the total invariant CMF energy squared $s=\left(p_{a}+p_{b}\right)^{2}$, the total invariant momentum transfer squared $t=\left(p_{b}-p_{d}\right)^{2}$, the invariant mass of the $\eta \pi$ system $m_{\eta \pi}=\sqrt{\left(p_{1}+p_{2}\right)^{2}}$, the invariant momentum transfer between the initial $\pi$ and final $\eta, t_{\eta \pi}=\left(p_{a}-p_{1}\right)^{2}$, and the invariant mass between the final state $\pi$ and $N, s_{\pi N}=\left(p_{2}+p_{d}\right)^{2}$. We will see below that we can trade two of these invariants for the orientation of the $\eta$-meson, which will be useful for partial wave analysis.

We choose to work in the Gottfried-Jackson (GJ) frame [58], which is the rest frame of the final state $\eta \pi$ system. The nucleon helicities are defined in this frame. In this chapter, we will denote all kinematic variables in the GJ frame via a $\star$, e.g. the total $\eta \pi$ momentum is $\mathbf{P}_{\eta \pi}^{\star}=\mathbf{p}_{1}^{\star}+\mathbf{p}_{2}^{\star}=\mathbf{0}$. We define the GJ $z$-axis along the direction of the initial $\pi$-beam, $\mathbf{z} \| \mathbf{p}_{a}^{\star}$, and define GJ $x y$-plane as the plane in which the $\pi$-beam, target nucleon, and recoil nucleon all lie, $\mathbf{y} \| \mathbf{p}_{b}^{\star} \times \mathbf{p}_{d}^{\star}$. In the GJ frame, the produced $\pi$ recoils against the $\eta$ such that $\mathbf{p}_{1}^{\star}=-\mathbf{p}_{2}^{\star} \equiv \mathbf{q}^{\star}$, where the magnitude of the relative momentum $\mathbf{q}^{\star}$ is

$$
q^{\star}=\left|\mathbf{q}^{\star}\right|=\frac{1}{2 m_{\eta \pi}} \lambda^{1 / 2}\left(m_{\eta \pi}^{2}, m_{\eta}^{2}, m_{\pi}^{2}\right)
$$

where $\lambda(a, b, c)=a^{2}+b^{2}+c^{2}-2(a b+b c+c a)$ is the Källén kinematic function [59, and has an orientation $\hat{\mathbf{q}}^{\star}=\left(\theta^{\star}, \varphi^{\star}\right)$, where $\theta^{\star}$ is the polar angle with respect to the $z$-axis, and $\varphi^{\star}$ is the azimuthal angle.

The reaction occurs at a fixed beam energy of $P_{\text {lab }}=190 \mathrm{GeV} / c$, which implies that the total 
invariant CMF energy is

$$
s=m_{\pi}^{2}+m_{N}^{2}+2 m_{N} \sqrt{m_{\pi}^{2}+P_{\mathrm{lab}}^{2}} \approx 380 \mathrm{GeV}^{2} .
$$

At such energies, the $\eta \pi$ system is produced diffractively, i.e., the system is dominated by Pomeron $(\mathbb{P})$ exchange $[60]$, and no quantum numbers are exchanged with the target. The produced $\eta \pi$ system are produced nearly collinearly with the beam, and is in the resonance region, i.e. $\left(m_{\eta}+\right.$ $\left.m_{\pi}\right) \leq m_{\eta \pi} \lesssim 2 \mathrm{GeV}$. Therefore, we assume this system is in the singe Regge limit, which is where $s, s_{\pi N} \rightarrow \infty$, while $s_{\pi N} / s$ is fixed. The energies of the five particles, in terms of invariants, are

$$
\begin{aligned}
& \omega_{a}^{\star}=\frac{m_{\eta \pi}^{2}+m_{\pi}^{2}-t}{2 m_{\eta \pi}} \\
& \omega_{b}^{\star}=\frac{s-m_{\pi}^{2}-m_{N}^{2}+t}{2 m_{\eta \pi}} \longrightarrow \frac{s}{2 m_{\eta \pi}} \\
& \omega_{d}^{\star}=\frac{s-m_{\eta \pi}^{2}-m_{N}^{2}}{2 m_{\eta \pi}} \longrightarrow \frac{s}{2 m_{\eta \pi}} \\
& \omega_{1}^{\star}=\frac{m_{\eta \pi}^{2}+m_{\eta}^{2}-m_{\pi}^{2}}{2 m_{\eta \pi}} \\
& \omega_{2}^{\star}=\frac{m_{\eta \pi}^{2}+m_{\pi}^{2}-m_{\eta}^{2}}{2 m_{\eta \pi}},
\end{aligned}
$$

where the asymptotic forms in Eqs. $3.4 \mathrm{~b}$ ) and $3.4 \mathrm{c}$ reflect the single Regge limit. The invariant $t_{\eta \pi}$ is related to the polar angle $\theta^{\star}$ via

$$
\begin{aligned}
t_{\eta \pi} & =m_{\pi}^{2}+m_{\eta}^{2}-\frac{1}{2 m_{\eta \pi}^{2}}\left(m_{\eta \pi}^{2}+m_{\pi}^{2}-t\right)\left(m_{\eta \pi}^{2}+m_{\eta}^{2}-m_{\pi}^{2}\right) \\
& +\frac{1}{2 m_{\eta \pi}^{2}} \lambda^{1 / 2}\left(m_{\eta \pi}^{2}, m_{\pi}^{2}, t\right) \lambda^{1 / 2}\left(m_{\eta \pi}^{2}, m_{\eta}^{2}, m_{\pi}^{2}\right) \cos \theta^{\star}
\end{aligned}
$$

and the invariant $s_{\pi N}$ is related to the azimuthal angle, which in the single Regge limit, one finds

$$
\begin{aligned}
\frac{2 m_{\eta \pi}^{2} s_{\pi N}}{s} & =m_{\eta \pi}^{2}+m_{\eta}^{2}-m_{\eta}^{2}+\frac{\lambda^{1 / 2}\left(m_{\eta \pi}^{2}, m_{\pi}^{2}, m_{\eta}^{2}\right)}{\lambda^{1 / 2}\left(m_{\eta \pi}^{2}, m_{\pi}^{2}, t\right)}\left(m_{\pi}^{2}-m_{\eta \pi}^{2}-t\right) \cos \theta^{\star} \\
& +\frac{\lambda^{1 / 2}\left(m_{\eta \pi}^{2}, m_{\pi}^{2}, m_{\eta}^{2}\right)}{\lambda^{1 / 2}\left(m_{\eta \pi}^{2}, m_{\pi}^{2}, t\right)} \sqrt{-4 t m_{\eta \pi}^{2}} \sqrt{1-\cos ^{2} \theta^{\star}} \cos \varphi^{\star}
\end{aligned}
$$

High-energy diffractive production allows us to assume factorization the amplitude into a a nuclear vertex and a $\pi \mathbb{P} \rightarrow \eta \pi$ amplitude, which resembles an ordinary helicity amplitude 61. 
It is a function of $m_{\eta \pi}$ and $t_{\eta \pi}$, as well as the total energy and the momentum transfer between the nucleon target and recoil. In the GJ frame, the Pomeron helicity in $\pi \mathbb{P} \rightarrow \eta \pi$ equals the $\eta \pi$ total angular momentum projection $M$, and the helicity amplitudes $a_{M}\left(s, t, t_{1}\right)$ can be expanded in partial waves $a_{J M}(s, t)$ with total angular momentum $J=L$. The allowed quantum numbers of the $\eta \pi$ partial waves are $J^{P}=1^{-}, 2^{+}, 3^{-}, \ldots$ The Pomeron exchange has natural parity. Parity relates the amplitudes with opposite spin projections, e.g. $\mathcal{A}^{J M}=-\mathcal{A}^{J-M}[62$. That is, the $M=0$ amplitude is forbidden and the two $M= \pm 1$ amplitudes are given, up to a sign, by a single scalar function.

The assumption about the Pomeron dominance can be quantified by the magnitude of unnatural partial waves. In the analysis of ref. [15], the magnitude of the $L=M=0$ wave was estimated to be $<1 \%$, and it also absorbs other possible reducible backgrounds. The patterns of azimuthal dependence in the central production of mesons [63 67] indicate that at low momentum transfer, $t \sim 0$, the Pomeron behaves as a vector 68,69], which is in agreement with the strong dominance of the $|M|=1$ component in the COMPASS data. 1 We are unable to further address the nature of the exchange from the data of ref. [15] since they are integrated over the momentum transfer $t 2^{2}$. However, from this single energy analysis we cannot be sure the exchange is purely Pomeron. Analyses such as Ref. 72 suggest there could be an $f$ exchange, but for our analysis the natural parity exchanges will be similar, so we consider an effective Pomeron which may be a mixture of pure Pomeron and $f$. We note here that COMPASS has published data in the $3 \pi$ channel, which are binned both in $3 \pi$ invariant mass and momentum transfer $t[17]$.

The COMPASS mass-independent analysis [15] is restricted to partial waves with $L=1$ to 6 and $|M|=1$ (except for the $L=2$ where also the $|M|=2$ wave is taken into account). The lowest mass exchanges in the crossed channels of $\pi \mathbb{P} \rightarrow \eta \pi$ correspond to the $a$ (in the $t_{\eta \pi}$ channel) and

\footnotetext{
${ }^{1}$ At low $t$, the Pomeron trajectory passes through $J=1$, while at larger, positive $t$, the trajectory is expected to pass though $J=2$ where it would relate to the tensor glueball 70.71 .

${ }^{2}$ For example, Ref. 72 suggested a dominance of $f_{2}$ exchanges for $a_{2}(1320)$ production. To probe this, one should analyze the $t$ and total energy dependences.
} 


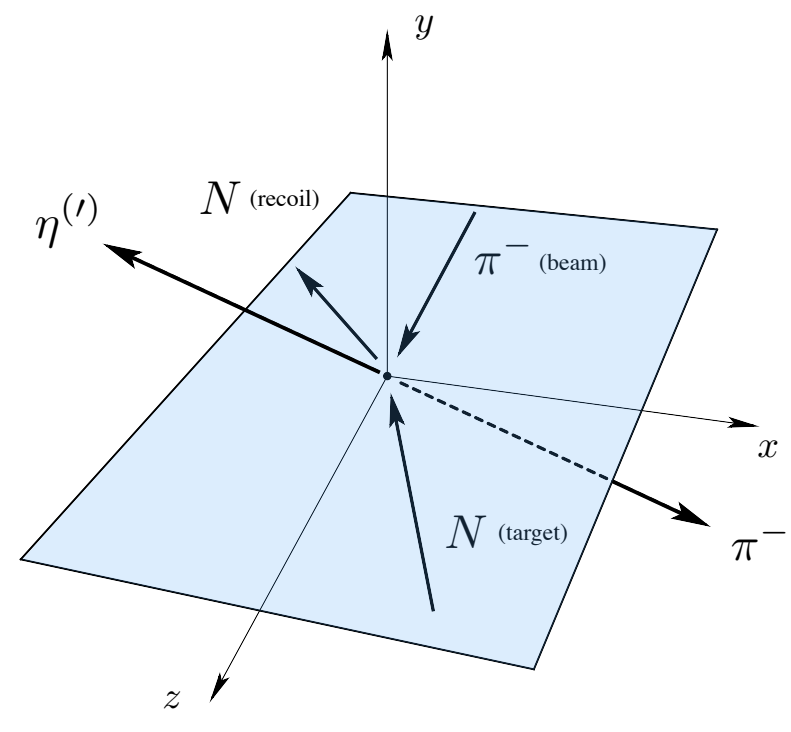

Figure 3.1: The reaction plane for $\eta^{-} N \rightarrow \eta \pi^{-} N$ in the Gottfried-Jackson (GJ) frame. The $\pi$ beam momentum is oriented alond the $z$-axis, with the nucleon target and recoil momenta lying in the $x z$-plane. The $\eta$ momentum has an orientation $\hat{\mathbf{q}}^{\star}=\left(\theta^{\star}, \varphi^{\star}\right)$ with respect to the $z$-axis.

the $f$ (in the $u_{\eta \pi}$ channel) trajectories, thus higher partial waves are not expected to be significant in the $\eta \pi$ mass region of interest, $m_{\eta \pi}<2 \mathrm{GeV}$. However, the systematic uncertainties associated with an analysis based on a truncated set of partial waves is hard to estimate.

The differential cross section in the $\eta \pi$ invariant mass is given by

$$
\begin{aligned}
\frac{\mathrm{d} \sigma}{\mathrm{d} m_{\eta \pi}} & =\frac{1}{4(4 \pi)^{4}}\left(\frac{\hbar c}{m_{N} P_{\mathrm{lab}}}\right)^{2} \sum_{J, M, \epsilon} \int_{t_{-}}^{t_{+}} \mathrm{d} t q^{\star} \sum_{\mu, \mu^{\prime}}\left|\mathcal{A}_{\mu^{\prime} \mu}^{J M \epsilon}\left(s, t, m_{\eta \pi}\right)\right|^{2} \\
& \equiv \sum_{J, M, \epsilon} \mathcal{I}^{J M \epsilon}\left(s, m_{\eta \pi}\right)
\end{aligned}
$$

where we define $\mathcal{I}^{J M \epsilon}$ as the intensity distribution, which corresponds to the quantity measured at COMPASS. The amplitude is expanded in the reflectivity basis [62],

$$
\mathcal{A}_{\mu^{\prime} \mu}\left(s, t, m_{\eta \pi}\right)=\sum_{J, M, \epsilon} \mathcal{A}_{\mu^{\prime} \mu}^{J M \epsilon}\left(s, t, m_{\eta \pi}\right) Y_{J M}^{\epsilon}\left(\theta^{\star}, \varphi^{\star}\right)
$$

where the reflectivity basis spherical harmonics are defined via the linear combination

$$
Y_{J M}^{\epsilon}\left(\theta^{\star}, \varphi^{\star}\right)=c_{M}\left[Y_{J M}\left(\theta^{\star}, \varphi^{\star}\right)-\epsilon P(-1)^{J-M} Y_{J-M}\left(\theta^{\star}, \varphi^{\star}\right)\right]
$$


where $c_{M}=1 / \sqrt{2}$ for $M=0$ and $c_{M}=1 / 2$ for $M \neq 0$, and the combination $P(-1)^{\ell}=\eta$ is the naturality of the state $c$, since recall $\ell=J$ for the two pseudoscalars in the final state. When $\eta=-\epsilon$, then $m_{\ell}=0$. The reflectivity-spherical harmonics are orthonormalized according to

$$
\int d \Omega Y_{\ell m_{\ell}}^{\epsilon}(\Omega) Y_{\ell^{\prime} m_{\ell}^{\prime}}^{\epsilon^{\prime}}(\Omega)=\delta_{\ell \ell^{\prime}} \delta_{m_{\ell} m_{\ell}^{\prime}} \delta_{\epsilon \epsilon^{\prime}}
$$

Two pseudoscalars can only form a state with positive naturality, so $\eta=P(-1)^{\ell}=+$ always. We can then write a simplified form for the reflectivity-spherical harmonics

$$
\begin{aligned}
& Y_{\ell m_{\ell}}^{+}(\Omega)=2 i c_{m} Y_{\ell m_{\ell}}(\theta, 0) \sin m_{\ell} \phi \\
& Y_{\ell m_{\ell}}^{-}(\Omega)=2 c_{m} Y_{\ell m_{\ell}}(\theta, 0) \cos m_{\ell} \phi
\end{aligned}
$$

Note that the reflectivity does not enter into the elastic partial wave amplitudes, as they are independent of the spin projection due to the Wigner-Eckart theorem. the reflectivity $\epsilon= \pm$ for bosons, and the spin projection is restricted $0 \leq m_{\ell} \leq \ell$. We choose the reflectivity $\epsilon$ to be equal to the naturality of the $t$-channel exchange for the $s$-channel $a+b \rightarrow c+d$, where the state $c$ is the (12) two-particle state with quantum numbers $J^{P C}$.

\subsubsection{PRODUCTION MODEL}

At zero momentum transfer, the Pomeron exchange has a Regge pole $\alpha_{\mathbb{P}}(0) \simeq 1$. Therefore, since we are interested in the small $t$-region, we will assume that the coupling of the effective Pomeron is that of a vector particle. This means that we will treat the spin-parity of our effective Pomeron as $J^{P}=1^{-}$. We denote the $\eta \pi$ resonance as $X$, where the resonance has spin-parity $J^{P}$, spin projection $M$, a mass $m_{\eta \pi}$, and a momentum $p_{c}=P_{\eta \pi}=p_{1}+p_{2}$. Assuming factorization of the $t$-channel Reggeon, we can write the production amplitude in terms of three vertices: The initial meson-production vertex $(\pi \mathbb{P} \rightarrow X)$, the final meson-production vertex $(X \rightarrow \eta \pi)$, and the nuclear $\operatorname{vertex}(N \rightarrow \mathbb{P} N)$. This is shown in Fig. 3.2 .

The initial meson-production vertex has the form

$$
\mathcal{V}_{\lambda_{\mathbb{P}} M}^{J}(\pi \mathbb{P} \rightarrow X)=\varepsilon^{\mu \nu \alpha \beta_{1}} p_{c}^{\mu} \epsilon_{\lambda_{\mathbb{P}}}^{\nu}\left(\mathbf{P}_{\mathbb{P}}\right) p_{a}^{\alpha} \epsilon_{M}^{\beta_{1} \cdots \beta_{J} *}\left(\mathbf{p}_{c}\right) p_{a}^{\beta_{2}} \cdots p_{a}^{\beta_{J}}
$$




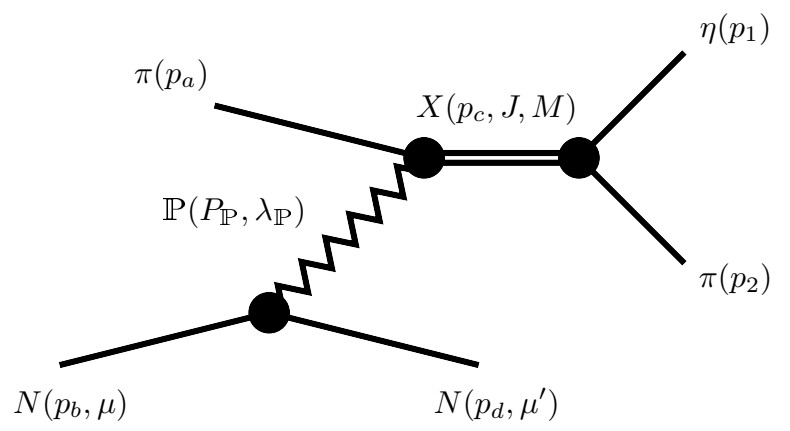

Figure 3.2: Diagram of $\eta \pi$ production where the system resonates in $J^{P} M$ quantum numbers, with parity $P=(-1)^{J}$.

where the momentum of the Pomeron is $P_{\mathbb{P}}=p_{c}-p_{a}$. The tensor $\varepsilon^{\mu \nu \alpha \beta_{1}}$ is the totally antisymmetric Levi-Civita tensor, and $\epsilon_{\lambda_{\mathbb{P}}}^{\nu}$ is the polarization tensor of the Pomeron. We can simplify the vertex by working in the GJ frame, $\mathbf{p}_{c}^{\star}=\mathbf{0}$. First note that for a polarization tensor of zero three-momentum with helicity $\lambda$ fully contracted with momenta $p$ with orientation $(\theta, \varphi)$, we can write the relation

$$
\epsilon_{\lambda}^{\mu_{1} \cdots \mu_{J}}(\mathbf{k}=\mathbf{0}) p^{\mu_{1}} \cdots p^{\mu_{J}}=\frac{1}{\sqrt{C_{J}}}\left|\mathbf{p}^{\star}\right|^{J} d_{\lambda 0}^{(J)}(\theta) e^{i \lambda \varphi},
$$

where $C_{J}=(2 J) ! 2^{-J} /(J !)^{2}[73]$. Second, a general polarization tensor for a spin $J$ can be written in terms of a Clebsch-Gordan series of two polarization tensors with spins $S$ and $J-S$ via

$$
\epsilon_{\lambda}^{\mu_{1} \cdots \mu_{J}}(\mathbf{p})=\sum_{\lambda_{1}, \lambda_{2}}\left\langle J \lambda \mid J-S \lambda_{2} ; S \lambda_{1}\right\rangle \epsilon_{\lambda_{1}}^{\mu_{1} \cdots \mu_{S}}(\mathbf{p}) \epsilon_{\lambda_{2}}^{\mu_{S+1} \cdots \mu_{J}}(\mathbf{p})
$$

Returning to the initial meson-production vertex, in the GJ frame, $p_{c}^{\star}=\left(m_{\eta \pi}, \mathbf{0}\right)$ and $\mathbf{p}_{a}=\left|\mathbf{p}_{a}^{\star}\right| \hat{\mathbf{z}}$. Therefore, using Eqs. (3.14) and (3.15), as well that $d_{\lambda 0}^{(J)}(0)=\delta_{\lambda 0}$, we arrive at the expression

$$
\mathcal{V}_{\lambda_{\mathbb{P}} M}^{J}(\pi \mathbb{P} \rightarrow X)=m_{\eta \pi} \frac{\left|\mathbf{p}_{a}^{\star}\right|^{J-1}}{\sqrt{C_{J-1}}}\langle J M \mid J-10 ; 1 M\rangle \boldsymbol{\epsilon}_{\lambda_{\mathbb{P}}}\left(\mathbf{P}_{\mathbb{P}}^{\star}\right) \cdot\left(\mathbf{p}_{a}^{\star} \times \boldsymbol{\epsilon}_{M}^{*}\left(\mathbf{p}_{c}^{\star}\right)\right),
$$

where we wrote the four-dimensional Levi-Civita as $\varepsilon^{0 \nu \alpha \beta}=\varepsilon_{j k l}$. Note that in the GJ frame, $\boldsymbol{\epsilon}_{M=0}^{*}\left(\mathbf{p}_{c}^{\star}\right)=(0,0,0,1)$, thus $\mathbf{p}_{a}^{\star} \| \boldsymbol{\epsilon}_{M=0}^{*}\left(\mathbf{p}_{c}^{\star}\right)$, the longitudinal component of the vertex is identically 
zero, i.e. $\quad M \neq 0$ for the resonance. Additionally, since $\boldsymbol{\epsilon}_{\lambda_{\mathbb{P}}}\left(\mathbf{P}_{\mathbb{P}}^{\star}\right) \cdot\left(\mathbf{p}_{a}^{\star} \times \boldsymbol{\epsilon}_{M}^{*}\left(\mathbf{p}_{c}^{\star}\right)\right)=\boldsymbol{\epsilon}_{M}^{*}\left(\mathbf{p}_{c}^{\star}\right)$. $\left(\boldsymbol{\epsilon}_{\lambda_{\mathbb{P}}}\left(\mathbf{P}_{\mathbb{P}}^{\star}\right) \times \mathbf{p}_{a}^{\star}\right)$, and $\mathbf{P}_{\mathbb{P}}^{\star}=-\mathbf{p}_{a}^{\star}$ with $\boldsymbol{\epsilon}_{\lambda_{\mathbb{P}}=0}\left(\mathbf{P}_{\mathbb{P}}^{\star}\right)=\left(\left|\mathbf{p}_{a}^{\star}\right|, 0,0, \omega_{\mathbb{P}}^{\star}\right) / M$, then the longitudinal component of the Pomeron is also zero. The final meson-production vertex contains a simpler coupling, as we consider only $J^{P} \rightarrow 0^{-} 0^{-}$, which has the form

$$
\begin{aligned}
\mathcal{V}_{M 0}^{J}(X \rightarrow \eta \pi) & =\epsilon_{M}^{\mu_{1} \cdots \mu_{J}}\left(\mathbf{p}_{c}\right) p_{1}^{\mu_{1}} \cdots p_{1}^{\mu_{J}} \\
& =\frac{1}{\sqrt{C_{J}}} q^{\star J} d_{M 0}^{(J)}\left(\theta^{\star}\right) e^{i M \varphi^{\star}}
\end{aligned}
$$

where we used Eq. 3.14 to evaluate the vertex in the GJ frame. Putting the two meson vertices together, we arrive at

$$
\begin{aligned}
\sum_{M=-J}^{J} \mathcal{V}_{\lambda_{\mathbb{P}} M}^{J}(\pi \mathbb{P} \rightarrow X) \mathcal{V}_{M 0}^{J}(X \rightarrow \eta \pi) & =\frac{m_{\eta \pi}}{\sqrt{C_{J} C_{J-1}}} q^{\star}\left|\mathbf{p}_{a}^{\star}\right|^{J-1} \sum_{j=1}^{3}\left(\boldsymbol{\epsilon}_{\lambda_{\mathbb{P}}}\left(\mathbf{P}_{\mathbb{P}}^{\star}\right) \times \mathbf{p}_{a}^{\star}\right)_{j} \\
& \times \sum_{M=-J}^{J}\langle J M \mid J-10 ; 1 M\rangle\left(\boldsymbol{\epsilon}_{M}^{*}\left(\mathbf{p}_{c}^{\star}\right)\right)_{j} d_{M 0}^{(J)}\left(\theta^{\star}\right) e^{i M \varphi^{\star}}
\end{aligned}
$$

which has the correct rotational dependence for a spin $J$ system.

The nuclear vertex has the structure

$$
\mathcal{V}_{\mu^{\prime} \mu ; \lambda_{\mathbb{P}}}(N \rightarrow \mathbb{P} N)=\epsilon_{\lambda_{\mathbb{P}}}^{\mu}\left(\mathbf{P}_{\mathbb{P}}\right) \bar{u}\left(\mathbf{p}_{d}, \mu^{\prime}\right) \gamma^{\mu} u\left(\mathbf{p}_{b}, \mu\right)
$$

and can be evaluated in a straightforward manner using properties of the Dirac spinors and Pauli matrices 74. Defining the angle of the target (and in the single Regge limit, recoil) angle with respect to the $z$-axis as $\alpha$, the result leads to

$$
\mathcal{V}_{\mu^{\prime} \mu ; \lambda_{\mathbb{P}}}(N \rightarrow \mathbb{P} N)=-\frac{s}{m_{\eta \pi}} \sum_{j=1}^{3} \boldsymbol{\epsilon}_{\lambda_{\mathbb{P}}}^{j}\left(\mathbf{P}_{\mathbb{P}}\right)\left[\delta_{j 1} \sin \alpha+\delta_{j 3} \cos \alpha\right] \Phi^{\top}\left(\mu^{\prime}\right) \Phi(\mu)
$$

where $\Phi(\mu)$ is the two component spinor, for which $\Phi^{\top}(+)=(1,0)$ and $\Phi^{\top}(-)=(0,1)$. Thus, the combination only allows for helicity non-flip systems, $\Phi^{\top}\left(\mu^{\prime}\right) \Phi(\mu)=\delta_{\mu^{\prime} \mu}$, which is consistent with Regge phenomenology in that Pomeron exchange conserves helicity. Since we showed that the Pomeron contains only a transverse component, only the $j=1$ term survives in Eq. (3.20). 
The nuclear vertex is therefore

$$
\mathcal{V}_{\mu^{\prime} \mu ; \lambda_{\mathbb{P}}}(N \rightarrow \mathbb{P} N)=-\frac{s}{m_{\eta \pi}} \boldsymbol{\epsilon}_{\lambda_{\mathbb{P}}}^{1}\left(\mathbf{P}_{\mathbb{P}}\right) \sin \alpha \delta_{\mu^{\prime} \mu}
$$

It is straightforward to show the in the Regge limit of the GJ frame,

$$
\begin{aligned}
\cos \alpha & =\longrightarrow \frac{-m_{\eta \pi}^{2}+m_{\pi}^{2}-t}{\lambda^{1 / 2}\left(m_{\eta \pi}^{2}, m_{\pi}^{2}, t\right)} \\
\sin \alpha & =\sqrt{1-\cos ^{2} \alpha} \longrightarrow \frac{2 m_{\eta \pi} \sqrt{-t}}{\lambda^{1 / 2}\left(m_{\eta \pi}^{2}, m_{\pi}^{2}, t\right)} .
\end{aligned}
$$

Putting all the tensor structures together, the production amplitude has the form

$$
\mathcal{A}_{\mu^{\prime} \mu}^{J}=-\frac{2 s}{\sqrt{2}} \frac{\left(\left|\mathbf{p}_{a}^{\star}\right|\left|\mathbf{p}_{1}^{\star}\right|\right)^{J}}{\sqrt{C_{J} C_{J-1}}}\langle J 1 \mid J-10 ; 11\rangle \sin \alpha \sin \varphi^{\star} d_{10}^{(J)}\left(\theta^{\star}\right) \delta_{\mu^{\prime} \mu} .
$$

From Eq. (3.22b), we see that $\sin \alpha$ contributes an inverse power of the momenta $\left|\mathbf{p}_{a}^{\star}\right|$. Thus, we conclude that the threshold singularity structure of the amplitude has the form

$$
\mathcal{A}_{\mu^{\prime} \mu}^{J}=\left|\mathbf{p}_{1}^{\star}\right|^{J}\left|\mathbf{p}_{a}^{\star}\right|^{J-1} \widehat{\mathcal{A}}_{\mu^{\prime} \mu}^{J},
$$

where $\widehat{\mathcal{A}}_{\mu^{\prime} \mu}^{J}$ is a regular function near thresholds. Ignoring the resonance propagator, which we will discuss in the following section, the production amplitude contains no singularities in the physical region of the invariant mass $m_{\eta \pi}$, as expected from our discussion of particle exchanges.

\subsubsection{FINAL STATE INTERACTIONS}

We now turn our attention to the final state interactions of the $\eta \pi$ system. In the simple model described above, we imagined the $\eta \pi$ resonance to be described by a relativistic Breit-Wigner amplitude. For narrow, isolated resonances, this picture may be an accurate description. We introduce a more sophisticated representation for the final state interactions of $\eta \pi$, which include a broad or interfering resonances. We begin by defining the elastic $\eta \pi$ amplitude,

$$
\left\langle\eta\left(p_{1}^{\prime}\right) \pi\left(p_{2}^{\prime}\right)|T| \eta\left(p_{1}\right) \eta\left(p_{2}\right)\right\rangle=(2 \pi)^{4} \delta\left(P_{\eta \pi}^{\prime}-P_{\eta \pi}\right) \mathcal{M}\left(m_{\eta \pi}, \cos \theta^{\star}\right)
$$

where $P_{\eta \pi}=p_{1}+p_{2}$ is the initial total $\eta \pi$ four momentum, and $P_{\eta \pi}^{\prime}=p_{1}^{\prime}+p_{2}^{\prime}$ is the final total four momentum, $m_{\eta \pi}^{2}=\left(p_{1}+p_{2}\right)^{2}$ is the $\eta \pi$ invariant mass-squared, and $\cos \theta^{\star}=\hat{\mathbf{p}}_{1}^{\star} \cdot \hat{\mathbf{p}}_{1}^{\prime \star}$ is the 
cosine of the scattering angle in the rest-frame of $\eta \pi$. The amplitude has a partial wave expansion

$$
\mathcal{M}\left(m_{\eta \pi}, \cos \theta^{\star}\right)=16 \pi \sum_{J=0}^{\infty}(2 J+1) \mathcal{M}_{J}\left(m_{\eta \pi}\right) P_{J}\left(\cos \theta^{\star}\right),
$$

where $J$ is the total angular momentum of the $\eta \pi$ system. Here, we have introduced a factor of $16 \pi$ for convenience, so that two-body phase space approaches unity as $m_{\eta \pi} \rightarrow \infty$, as opposed to the convention used in the previous chapter.

Having defined the elastic $\eta \pi$ amplitude, we can now proceed to impose the unitarity condition to the $\eta \pi$ production amplitude in the $\eta \pi$-channel. At this point, we will assume that the only intermediate state allowed is $\eta \pi$, even though other kinematically allowed channels can couple to the resonances of interest. We will address the inclusion of additional states later. In the elastic approximation, the partial wave unitarity relation is

$$
\operatorname{Im} \mathcal{A}_{\mu^{\prime} \mu}^{J M \epsilon}\left(s, t, m_{\eta \pi}\right)=\frac{2 q^{\star}}{m_{\eta \pi}} \mathcal{M}_{J}^{*}\left(m_{\eta \pi}\right) \mathcal{A}_{\mu^{\prime} \mu}^{J M \epsilon}\left(s, t, m_{\eta \pi}\right) \Theta\left(m_{\eta \pi}-\left(m_{\eta}+m_{\pi}\right)\right)
$$

where we note that no additional numerical factors are included due to our normalization in Eq. (3.26). There is an additional threshold singularity in the partial wave amplitudes, which enforces that $\mathcal{M}_{J} \sim q^{\star 2 J}$ as $m_{\eta \pi} \rightarrow\left(m_{\eta}+m_{\pi}\right)$. Therefore, we remove this singularity from the amplitudes, first defining

$$
\mathcal{M}_{J}\left(m_{\eta \pi}\right)=q^{\star 2 J} \widehat{\mathcal{M}}_{J}\left(m_{\eta \pi}\right)
$$

where $\widehat{\mathcal{M}}_{J}\left(m_{\eta \pi}\right)$ is regular near threshold. We have already seen the threshold structure of the production amplitude, Eq. (3.24), and we factor these singularities from Eq. (3.27), yielding

$$
\operatorname{Im} \widehat{\mathcal{A}}_{\mu^{\prime} \mu}^{J M \epsilon}\left(s, t, m_{\eta \pi}\right)=\rho_{J}\left(m_{\eta \pi}\right) \widehat{\mathcal{M}}_{J}^{*}\left(m_{\eta \pi}\right) \widehat{\mathcal{A}}_{\mu^{\prime} \mu}^{J M \epsilon}\left(s, t, m_{\eta \pi}\right) \Theta\left(m_{\eta \pi}-\left(m_{\eta}+m_{\pi}\right)\right)
$$

where we define

$$
\rho_{J}\left(m_{\eta \pi}\right)=\frac{2 q^{\star 2 J+1}}{m_{\eta \pi}} .
$$

From Eq. 3.29), the production amplitude must have the same phase as the elastic amplitude, which is a consequence of Watson's theorem. The elastic amplitude satisfies its own unitarity 
relation, which is simply the elastic unitarity relation

$$
\operatorname{Im} \widehat{\mathcal{M}}_{J}\left(m_{\eta \pi}\right)=\rho_{J}\left(m_{\eta \pi}\right)\left|\widehat{\mathcal{M}}_{J}\left(m_{\eta \pi}\right)\right|^{2} \Theta\left(m_{\eta \pi}-\left(m_{\eta}+m_{\pi}\right)\right) .
$$

As seen in the previous chapter, we can write the elastic $\eta \pi$ amplitude in the $N / D$ representation,

$$
\widehat{\mathcal{M}}_{J}\left(m_{\eta \pi}\right)=\mathcal{N}_{J}\left(m_{\eta \pi}\right) / \mathcal{D}_{J}\left(m_{\eta \pi}\right)
$$

where

$$
\mathcal{D}_{J}\left(m_{\eta \pi}\right)=\mathcal{D}_{J}^{(0)}\left(m_{\eta \pi}\right)-\frac{m_{\eta \pi}^{2}}{\pi} \int_{\left(m_{\eta}+m_{\pi}\right)^{2}}^{\infty} \mathrm{d} m^{2} \frac{\rho_{J}(m) \mathcal{N}_{J}(m)}{m^{2}\left(m^{2}-m_{\eta \pi}^{2}-i \epsilon\right)}
$$

where $\mathcal{D}_{J}^{(0)}$ is a real rational function in the physical region, which we represent as

$$
\mathcal{D}_{J}^{(0)}\left(m_{\eta \pi}^{2}\right)=c_{0}-c_{1} m_{\eta \pi}^{2}-\sum_{j=1} \frac{c_{2}^{(j)}}{c_{3}^{(j)}-m_{\eta \pi}^{2}}
$$

where $c_{j}$ for $j=0, \ldots 3$ are real parameters for each partial wave $J$, which are to be determined. In the following sections, we will apply our production model to investigate $\eta \pi$ resonances. Given the $\mathcal{N}_{J}$ function, which contains the particle exchange cuts, the $\mathcal{D}_{J}$ function can be solved. For simplicity, we will model $\mathcal{N}_{J}$ with physics representing the unknown exchange physics, and some unknown parameters to be determined. A simple model which we will use in our analysis of COMPASS data is

$$
\rho_{J}\left(m_{\eta \pi}\right) \mathcal{N}_{J}\left(m_{\eta \pi}\right)=\frac{\lambda^{J+1 / 2}\left(m_{\eta \pi}^{2}, m_{\eta}^{2}, m_{\pi}^{2}\right)}{\left(m_{\eta \pi}^{2}+\Lambda_{R}\right)^{2 J+1+\gamma}}
$$

where $\Lambda_{R}$ effectively describes the radius of interactions.

\subsubsection{ANALYSIS OF TENSOR RESONANCES}

As a first test of the model, we investigate tensor $(J=2)$ resonances in the $\eta \pi$ system, where the well-known $a_{2}(1320)$ meson is featured as a vivid peak in the $\eta \pi$ mass distribution. The $\eta \pi$ $D$-wave is important to understand as it is used as a reference wave for all other waves measured at COMPASS. Furthermore, the first $a_{2}$ excitation is considerably more difficult to measure compared to the ground state $a_{2}$, thus giving us the opportunity to use our model to determine the excited 
state properties. There is a noticeable, but shallow, bump in the COMPASS data occurring at $\sim 1700 \mathrm{MeV}$, which is the approximate mass for the $a_{2}^{\prime}$ as measured in other experiments.

To compare with the partial wave intensities measured in Ref. [15], which are integrated over $t$ from $t_{\min }=-1.0 \mathrm{GeV}^{2}$ to $t_{\max }=-0.1 \mathrm{GeV}^{2}$, we use an effective value for the momentum transfer $t_{\text {eff }}=-0.1 \mathrm{GeV}^{2}$ and $\mathcal{A}_{J M}\left(m_{\eta \pi}\right) \equiv \mathcal{A}_{J M}\left(m_{\eta \pi}, s, t_{\mathrm{eff}}\right)$. The effect of a possible $t_{\text {eff dependence is }}$ taken into account in the estimate of the systematic uncertainties. The natural parity exchange partial wave amplitudes $\mathcal{A}_{J M}(s)$ can be identified with the amplitudes $A_{L M}^{\epsilon=1}(s)$ as defined in Eq. (1) of Ref. [15], where $\epsilon=+1$ is the reflectivity eigenvalue that selects the natural parity exchange.

In the following we consider the single, $J=2,|M|=1$ natural parity partial wave, which we denote its intensity (acceptance corrected) [15] as

$$
\mathcal{I}\left(m_{\eta \pi}\right) \equiv N q^{\star}\left|\mathcal{A}\left(m_{\eta \pi}\right)\right|^{2}
$$

Since the physical normalization of the cross section is not determined in Ref. [15], the constant $N$ is a normalization constant, which effectively absorbs all energy independent factors of the differential cross section. The overall scale is fixed to $N=10^{6}$, so that the coefficients $\alpha_{j}$ are expected to be $\mathcal{O}(1)$.

In principle, one should consider the coupled-channel problem involving all the kinematically allowed intermediate states (see Fig. ??). The PDG reports the important final states for the $2^{++}$system are the $3 \pi\left(\rho \pi, f_{2} \pi\right)$ and $\eta \pi$ systems $[6]$. Far from thresholds, a narrow peak in the data is generated by a pole in the closest unphysical sheet, regardless of the number of open channels. The residues (related to the branching ratios) depend on the individual couplings of each channel to the resonance, and therefore their extraction requires the inclusion of all the relevant channels. However, the pole position is expected to be essentially insensitive to the inclusion of multiple channels. This is easily understood in the Breit-Wigner approximation, where the total width extracted for a given state is independent of the branchings to individual channels. Thus, when investigating the pole position, we restrict the analysis to the elastic approximation, where only $\eta \pi$ can appear in the intermediate state. We will elaborate on the effects of introducing the 
$\rho \pi$ channel, which is known to be the dominant one of the decay of $a_{2}(1320)$ [6], as part of the systematic checks.

The production model proposed does not allow for flexibility in trying to fit the line shape. So, in order to have a flexible model, we replace the production model with an expansion in the invariant mass $m_{\eta \pi}$. This substitution allows us to capture additional background effects not modeled. One requirement is that the production mechanism is still a smooth function in the physical region. This can be seen by solving the unitarity constraint Eq. (3.29),

$$
\begin{aligned}
\widehat{\mathcal{A}}_{\mu^{\prime} \mu}^{J M \epsilon}\left(s, t, m_{\eta \pi}\right) & =\mathcal{B}_{\mu^{\prime} \mu}^{J M \epsilon}\left(s, t, m_{\eta \pi}\right) \\
& +\frac{1}{\mathcal{D}_{J}\left(m_{\eta \pi}\right)} \int_{\left(m_{\eta}+m_{\pi}\right)^{2}}^{\infty} \frac{\mathrm{d} m^{2}}{\pi} \frac{\rho_{J}(m) \mathcal{N}_{J}(m)}{m^{2}-m_{\eta \pi}^{2}-i \epsilon} \mathcal{B}_{\mu^{\prime} \mu}^{J M \epsilon}(s, t, m)
\end{aligned}
$$

which is the well known Omnes-Muskhelishvili solution. The function $\mathcal{B}_{\mu^{\prime} \mu}^{J M \epsilon}\left(s, t, m_{\eta \pi}\right)$ is a driving term which contains no singularities in the $\eta \pi$ physical region and does contain exchange cuts. If $\mathcal{B}_{\mu^{\prime} \mu}^{J M \epsilon}$ is specified, along with $\mathcal{N}_{J}$, then Eq. 3.37 gives the full amplitude. We could determine $\mathcal{B}_{\mu^{\prime} \mu}^{J M \epsilon}$ from our production model, but instead we will absorb all production physics in the expansion

$$
\mathcal{A}\left(s, t_{\mathrm{eff}}, m_{\eta \pi}\right)=q^{\star J} k^{\star J-1} \mathcal{P}\left(m_{\eta \pi}\right) / \mathcal{D}_{J=2}\left(m_{\eta \pi}\right),
$$

where the production expansion $\mathcal{P}$ is

$$
\mathcal{P}\left(m_{\eta \pi}\right)=\frac{1}{c_{3}-m_{\eta \pi}^{2}} \sum_{j=0}^{n} \alpha_{j} T_{j}\left(\omega\left(m_{\eta \pi}\right)\right),
$$

where the expansion contains the CDD pole at $c_{3}$ in order to cancel the forced zero from the $\mathcal{D}_{J}$ function, $\alpha_{j}$ are expansion coefficients which are to be determined, and $T_{n}$ are Chebyshev polynomials ${ }^{3}$ of the function

$$
\omega\left(m_{\eta \pi}\right)=\frac{m_{\eta \pi}^{2}}{m_{\eta \pi}^{2}+m_{0}^{2}},
$$

\footnotetext{
${ }^{3}$ The Chebyshev polynomial are expressed by the recursion relation

$$
T_{n}(z)=2 z T_{n-1}(z)-T_{n-2}(z) \quad \text { for } n \geq 2
$$

with $T_{0}(z)=1$, and $T_{1}(z)=z$.
} 
which is a pole approximation for the left-hand cut, where we choose the pole at $m_{0}^{2}=1 \mathrm{GeV}^{2}$. We choose the expansion in Chebyshev polynomials as opposed to standard polynomials in order to break correlations, which we discuss later in this section.

We fit a set of parameters $\boldsymbol{\theta}=\left(\theta_{1}, \ldots, \theta_{n}\right)$ to the COMPASS data via a $\chi^{2}$ minimization, where the $\chi^{2}$ is given by

$$
\chi^{2}(\boldsymbol{\theta})=\sum_{j=1}^{N_{\text {data }}}\left(\frac{\mathcal{I}_{\text {model }}\left(m_{\eta \pi}^{(j)} \mid \boldsymbol{\theta}\right)-\mathcal{I}_{\text {data }}(j)}{\sigma_{\text {data }}(j)}\right)^{2}
$$

where the $\mathcal{I}_{\text {data }}(j) \pm \sigma_{\text {data }}(j)$ is the intensity measured by COMPASS in $\eta \pi$ mass bins $m_{\eta \pi}^{(j)}$. The minimization, $\boldsymbol{\nabla}_{\boldsymbol{\theta}} \chi^{2}(\boldsymbol{\theta})=0$, yields the optimal set of parameters $\boldsymbol{\theta}$ to describe the available COMPASS data. We fit our model to the intensity distribution for $\pi^{-} p \rightarrow \eta \pi^{-} p$ in the $D$-wave (56 data points) [15], as defined in Eq. 3.36), by minimizing $\chi^{2}$. We fit the coefficients $a_{j}$ (see Eq. (3.39) , which are then expected to be $\mathcal{O}(1)$, and also the parameters in the $\mathcal{D}\left(m_{\eta \pi}\right)$ function. In the first step we obtain the best fit for a given total number of parameters, and in the second step we estimate the statistical uncertainties using the bootstrap technique [?,55 57]. That is to say, we generate $10^{5}$ pseudodata sets, each data point being resampled according to a Gaussian distribution having as mean and standard deviation the original value and error, and we repeat the fit for each set. In this way, we obtain $10^{5}$ different values for the fit parameters, and we take the means and standard deviations as expected values and statistical uncertainties, respectively.

In order to assess the systematic uncertainties we study the dependence of the pole parameters on variations of the model, specifically we change $i$ ) the number of CDD poles from 1 to 3 , ii) the total number of terms $n$ in the expansion of the numerator function $\mathcal{P}(s)$ in Eq. $(3.39)$, iii) the value of $\Lambda_{R}$ in the left hand cut model, $\left.i v\right)$ the value of $t_{\text {eff }}$ of the total momentum transferred, and $v$ ) the addition of the $\rho \pi$ channel to study coupled-channel effects. The fit with $\mathrm{CDD}_{\infty}$ only, shown in Fig. 3.4 , for $\Lambda_{R}=1.5 \mathrm{GeV}^{2}$ and $n=6$ (with a total of 9 parameters), captures neither the dip at $1.5 \mathrm{GeV}$ nor the bump at $1.7 \mathrm{GeV}$. Figure 3.3 shows the $\chi^{2}$ as a function of $\Lambda_{R}$, in which the minimum corresponds to $1.5 \mathrm{GeV}^{2}$. In contrast, the fit with two CDD poles (11 parameters), shown in Fig. 3.4 , captures both features, giving a $\chi^{2} /$ dof $=86.17 /(56-11)=1.91$. The parameters 


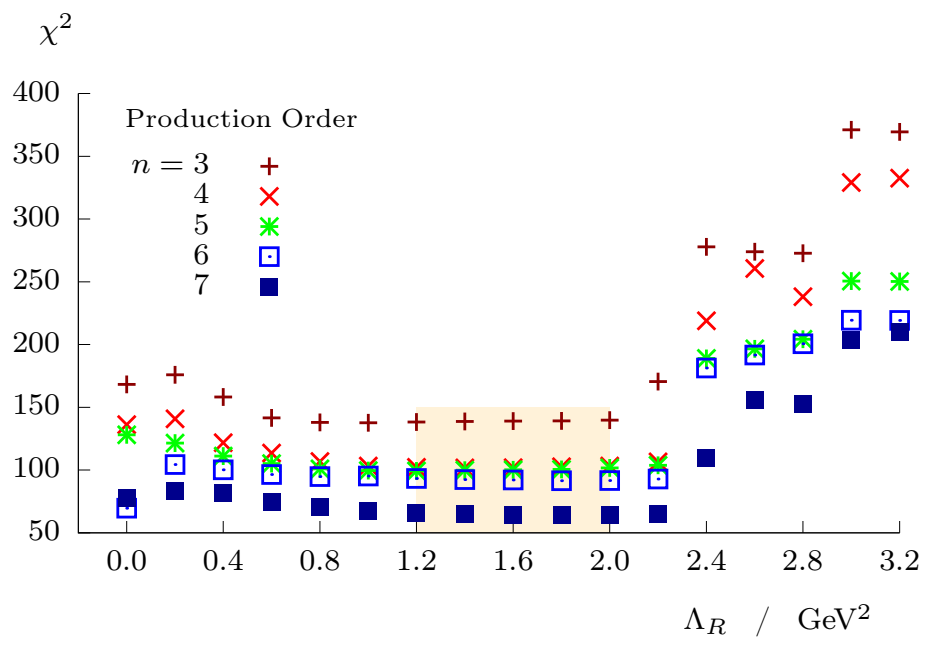

Figure 3.3: Preliminary $\chi^{2}$ fit results as a function of $\Lambda_{R}$ and the number of terms in the production expansion. These preliminary runs were used to determine the optimal value for the interaction radius $\Lambda_{R}$, which was determined to be within the range $1.2 \mathrm{GeV}^{2} \leq \Lambda_{R} \leq 2.0 \mathrm{GeV}^{2}$ (shown in the orange highlighted box). Note that for $\Lambda_{R}=0 \mathrm{GeV}^{2}$ as this yields unphysical singularities close to the physical region.

corresponding to the best fit with two CDD poles are given in Table 3.1. The addition of another CDD pole does not improve the fit, as the limited precision in the data is incapable of indicating any further resonances. Specifically the residue of the additional pole turns out to be compatible with zero, leaving the other fit parameters unchanged. We associate no systematic uncertainty to that.

As discussed earlier, an acceptable numerator function $n(s)$ should be "smooth" in the resonance region, i.e. without significant peaks or dips on the scale of the resonance widths. The parameters $c_{i}$ and $g$ of the denominator function are related to resonance parameters, while $s_{R}$ controls the distant second sheet singularities due to exchange forces. The expansion in $n(s)$, shown in for $s_{R}=1.5 \mathrm{GeV}^{2}$ and two CDD poles, has a singularity occurring at $s=-1.0 \mathrm{GeV}^{2}$ because of the definition of $\omega(s)$ and our choice of $\Lambda$. For variations in $n(s)$ between $n_{p}=3$ and $n_{p}=7$,

\footnotetext{
${ }^{4}$ Note that the production term is not well constrained below $s \sim 1 \mathrm{GeV}^{2}$, as the phase space and barrier factors highly suppress the near threshold behavior. The singularity at $s=-1 \mathrm{GeV}^{2}$ however, persist for each $n_{p}$ solution.
} 

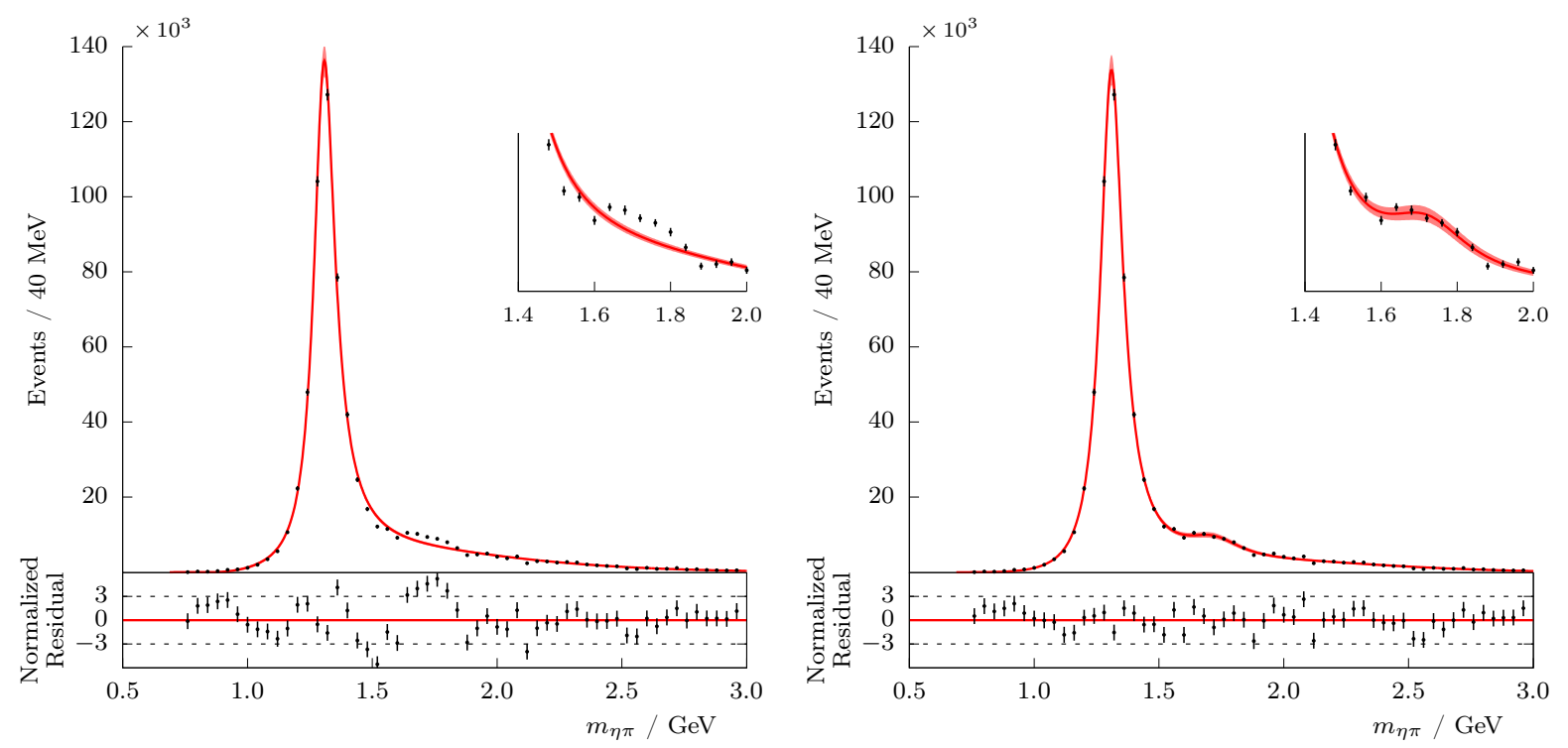

Figure 3.4: Intensity distribution and fits to the $J^{P C}=2^{++}$wave for different number of CDD poles, (a) using only $\mathrm{CDD}_{\infty}$ and (b) using $\mathrm{CDD}_{\infty}$ and the $\mathrm{CDD}$ pole at $m_{\eta \pi}^{2}=c_{3}$. Red lines are fit results with $\mathcal{I}\left(m_{\eta \pi}\right)$ given by Eq. (3.36). Data is taken from Ref. [15]. The inset shows the $a_{2}^{\prime}$ region. The error bands correspond to the $3 \sigma(99.7 \%)$ confidence level. Figure adapted from [2]. 


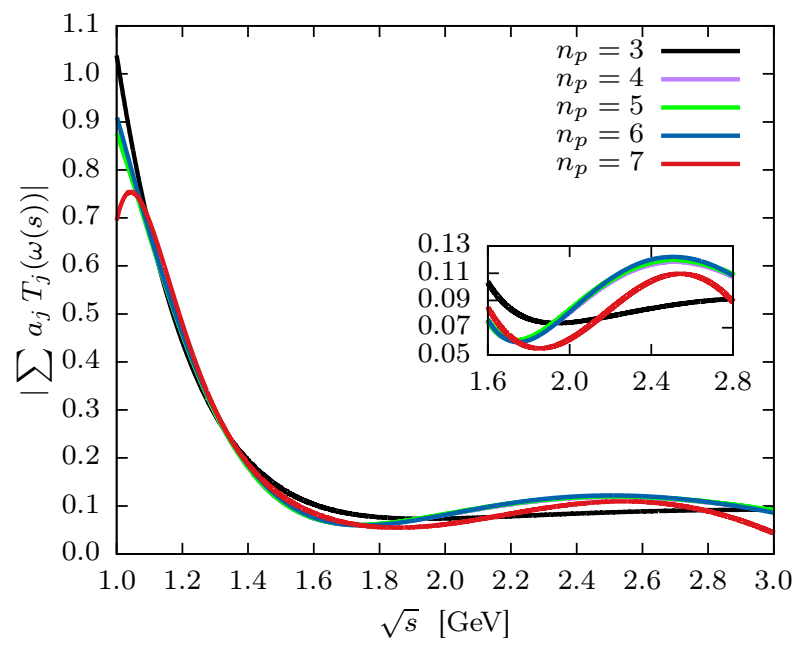

Figure 3.5: Amplitude numerator function $\left|\sum_{j}^{n} a_{j} T_{j}(\omega(s))\right|$ for different values of $n_{p}$. The absolute value is taken as there is a phase ambiguity because we fit only the intensity $\sim|\mathcal{A}(s)|^{2}$. Note that each curve is an independent fit for a specific number of terms $n$. The curves for $n=4,5$, and 6 all coincide in the resonance region, as shown in the inset.

we find the pole positions are relatively stable, which we discuss later in our systematic estimates.

The dependence on $t_{\text {eff }}$ is expected to affect mostly the overall normalization. Indeed, the variation from $t_{\mathrm{eff}}=-1.0 \mathrm{GeV}^{2}$ to $-0.1 \mathrm{GeV}^{2}$ gives less than $2 \%$ difference for the $a_{2}^{\prime}(1700)$ parameters, and $<1 \%$ for the $a_{2}(1320)$, and can be neglected compared to the other uncertainties.

We used both a polynomial expansion and a Chebyshev polynomial expansion. Both studies reach the same results regarding the $a_{2}^{(\prime)}$ poles extraction. Hence, it is clear from these studies that the resonance poles are independent of the production mechanism. In Table ?? we provide the correlation matrix for a standard polynomial, while Table ?? shows the correlation matrix for Chebyshev polynomials. If we focus on the polynomial expansion parameters (i.e. $\left.a_{0} \ldots a_{6}\right)$ we see that the correlations are essentially \pm 1 for the standard polynomials. For Chebyshev there is some improvement, although still large. For the standard polynomial expansion, the resonance parameters $\left(g, c_{1}, c_{2}, c_{3}\right)$, stay more or less independent of the production coefficients. However, 

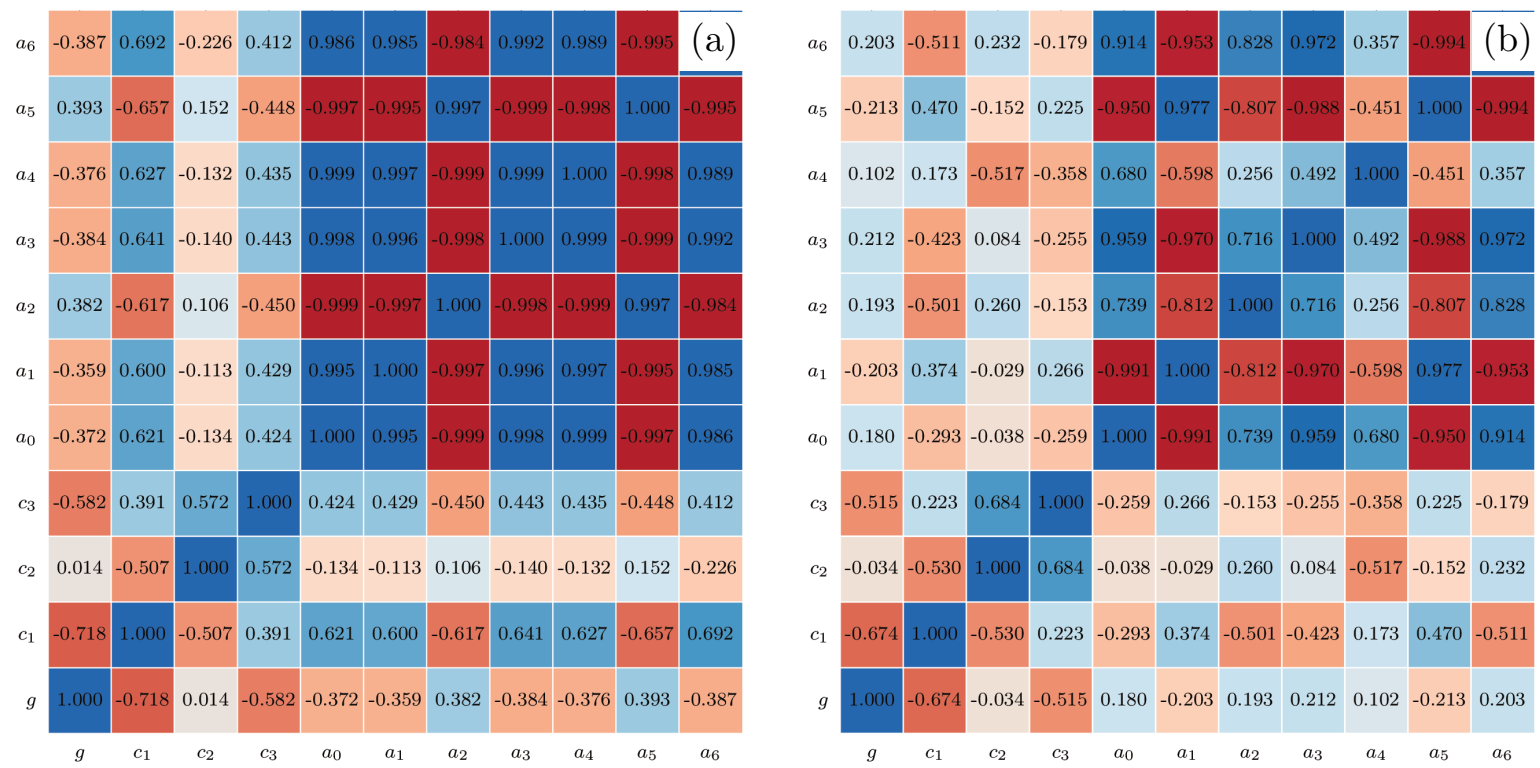

Figure 3.6: Correlation matrices for the fit with two CDD poles and $n=6$ terms in the production expansion, with a resulting $\chi^{2} /$ dof $=1.91$. The polynomial expansion (a) shows extremely large correlations in the production parameters, essentially \pm 1 , while the Chebychev expansion (b), although still large, breaks up some of the correlation in the production expansion.

the correlation between production and resonance parameters are significantly reduced when we use Chebyshev polynomials. This indicates that by using Chebyshev polynomials the resonances extraction is more robust. Figure 3.6 shows the correlation matrix for the best fit.

\section{ANALYTIC CONTINUATION AND POLE DETERMINATION}

This analysis allows us to extract the $\eta \pi \rightarrow \eta \pi$ elastic amplitude in the $D$-wave. By construction, the amplitude has a zero at $s=c_{3}$. Figure 3.7 shows the real and imaginary parts of $\hat{\mathcal{M}}(s)$, with the $3 \sigma$ error bands estimated by the bootstrap analysis. Resonance poles are extracted by analytically continuing the denominator of the $\eta \pi$ elastic amplitude to the second Riemann sheet (II) across the unitarity cut using

$$
\mathcal{D}_{J=2}^{\mathrm{II}}\left(m_{\eta \pi}\right)=\mathcal{D}_{J=2}\left(m_{\eta \pi}\right)+2 i \rho_{J=2}\left(m_{\eta \pi}\right) \mathcal{N}_{J=2}\left(m_{\eta \pi}\right)
$$




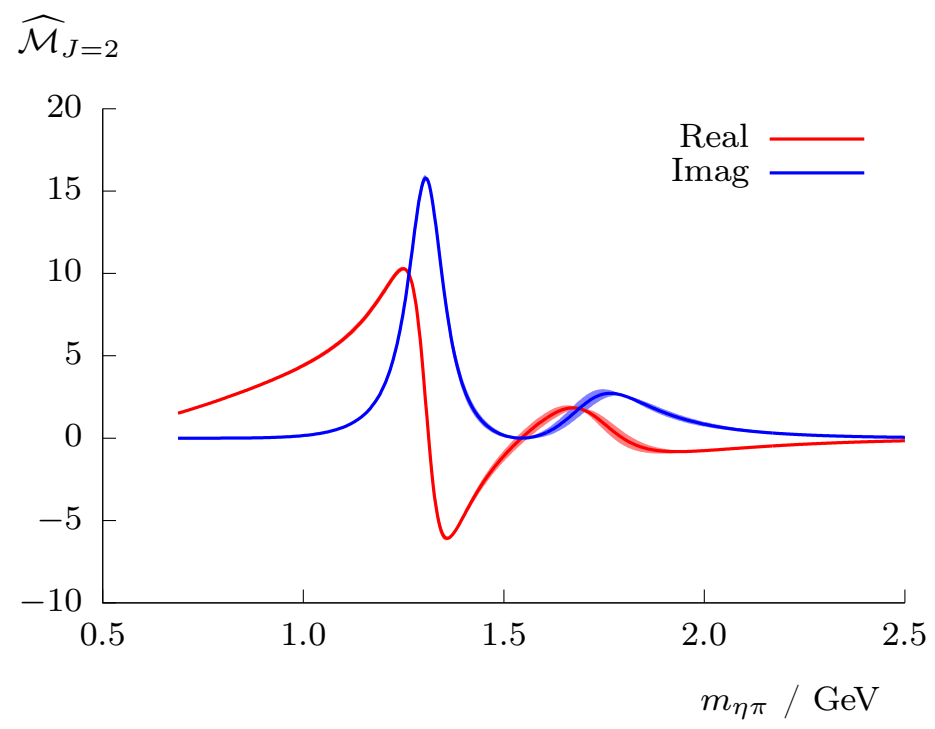

Figure 3.7: The reduced elastic $\eta \pi D$-wave amplitude, $\widehat{\mathcal{M}}_{J=2}=\mathcal{N}_{J=2} / \mathcal{D}_{J=2}$, as a function of the $\eta \pi$ invariant mass. Shown are the real (red) and imaginary (blue) parts with $3 \sigma$ a error band. The interaction range is fixed at $\Lambda_{R}=1.5 \mathrm{GeV}^{2}$. Figure adapted from $[2]$.

By construction, no first-sheet poles are present. We find three second-sheet poles in the energy range of $\left(m_{\pi}+m_{\eta}\right) \leq \sqrt{s} \leq 3 \mathrm{GeV}$, two of which can be identified as resonances, as shown in Fig. 3.8 for $n_{p}=6$ and $s_{R}=\{1.0,1.5,2.0,2.5\} \mathrm{GeV}^{2}$.

The mass and width are defined as $m=\operatorname{Re} \sqrt{s_{p}}$ and $\Gamma=-2 \operatorname{Im} \sqrt{s_{p}}$, respectively, where $s_{p}$ is the pole position in the $m_{\eta \pi}^{2}$-plane. Two of the poles found can be identified as the $a_{2}(1320)$ and $a_{2}^{\prime}(1700)$ resonances in the PDG $[6]$. The lighter of the two corresponds to the $a_{2}(1320)$. For $s_{R}=1.5 \mathrm{GeV}^{2}$, the pole has mass and width $m=(1307 \pm 1) \mathrm{MeV}$ and $\Gamma=(112 \pm 1) \mathrm{MeV}$, respectively. The nominal value is the best fit pole position, and the uncertainty is the statistical deviation determined from the bootstrap. Values of $s_{R}$ between 1.0 and $2.5 \mathrm{GeV}^{2}$ lead to pole deviations of at most $\Delta m=2 \mathrm{MeV}$ and $\Delta \Gamma=3 \mathrm{MeV}$. The heavier pole corresponds to the excited $a_{2}^{\prime}(1700)$. For $s_{R}=1.5 \mathrm{GeV}^{2}$, the resonance has mass and width $m=(1720 \pm 10) \mathrm{MeV}$ and $\Gamma=(280 \pm 10) \mathrm{MeV}$, respectively. The maximal deviations for the different $s_{R}$ values are $\Delta m=40 \mathrm{MeV}$ and $\Delta \Gamma=60 \mathrm{MeV}$. The $a_{2}(1320)$ and $a_{2}^{\prime}(1700)$ poles (see Fig. 3.8) are found to 


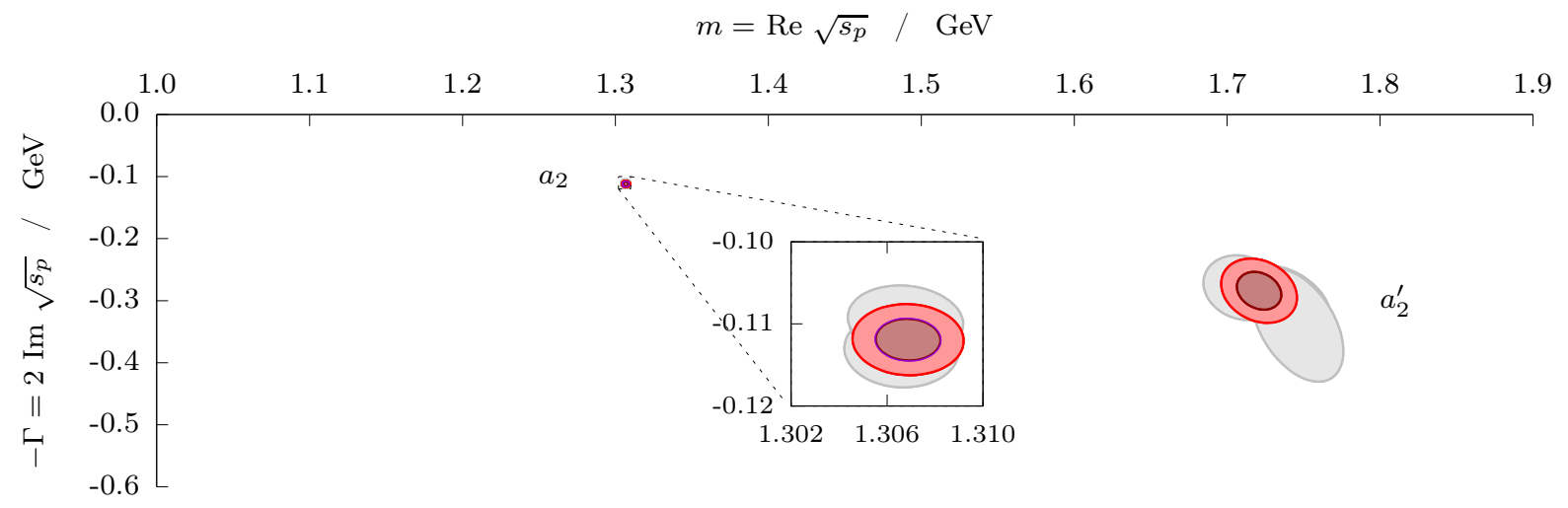

Figure 3.8: Location of second-sheet pole positions with two CDD poles and $n_{p}=6$ terms in the production expansion. Poles are shown with $1 \sigma$ (light red) and $2 \sigma$ (dark red) confidence levels from uncertainties computed using $10^{5}$ bootstrap fits. The gray ellipses show pole locations from systematic studies of varying $\Lambda_{R}$ from 1.0 to $2.5 \mathrm{GeV}^{2}$. Figure adapted from 2.

Table 3.1: Best fit denominator and production parameters for the fit with two CDD poles, $\Lambda_{R}=$ $1.5 \mathrm{GeV}^{2}, N=10^{6}, c_{0}=(1.23)^{2}$, and the number of expansion parameters $n_{p}=6$, leading to $\chi^{2} /$ dof $=1.91$. Denominator uncertainties are determined from a bootstrap analysis using $10^{5}$ random fits. We report no uncertainties on the production parameters as they are highly correlated.

\begin{tabular}{ccc|lc}
\multicolumn{2}{c|}{ Denominator parameters } & \multicolumn{2}{|l}{ Production parameters } & {$\left[\mathrm{GeV}^{-2}\right]$} \\
\hline$c_{1}$ & $0.532 \pm 0.006$ & $\mathrm{GeV}^{-2}$ & $\alpha_{0}$ & 0.471 \\
$c_{2}$ & $0.253 \pm 0.007$ & $\mathrm{GeV}^{2}$ & $\alpha_{1}$ & 0.134 \\
$c_{3}$ & $2.38 \pm 0.02$ & $\mathrm{GeV}^{2}$ & $\alpha_{2}$ & -1.484 \\
$g$ & $113 \pm 1$ & $\mathrm{GeV}^{4}$ & $a_{3}$ & 0.879 \\
& & & $\alpha_{4}$ & 2.616 \\
& & $\alpha_{5}$ & -3.652 \\
& & & $\alpha_{6}$ & 1.821
\end{tabular}

be stable under variations of $s_{R}$, which modulates the left hand cut.

As expected, there is a third pole that depends strongly on $\Lambda_{R}$ and reflects the singularity in $\mathcal{N}(s)$ modeled as a pole. Its mass ranges from 1.4 to $3.3 \mathrm{GeV}$, and its width varies between 1.3 and 
$1.8 \mathrm{GeV}$ as $\Lambda_{R}$ changes from $1 \mathrm{GeV}^{2}$ to $2.5 \mathrm{GeV}^{2}$. In the limit $g \rightarrow 0$, this pole moves to $-s_{R}$ as expected, while the other two migrate to the real axis above threshold 75. Fig. 3.9 shows many poles in the complex $s$-plane, three of which have $\operatorname{Re} s>0$, and two for $\operatorname{Re} s<0$. We want to understand the nature of all these singularities, primarily in identifying if these poles correspond to QCD resonances. We see two poles (corresponding to the $a_{2}$ and $a_{2}^{\prime}$ resonances) migrate to the real axis as the coupling is turned off, which is expected for the two CDD poles we chose. The other poles all migrate to $s=-1.5 \mathrm{GeV}^{2}$, which is the location of the left-hand singularity in our model. This shows clearly the origin of the poles. Fig. 3.9 shows snapshots at different values of $g$ as $g \rightarrow 0$. The poles that tend to $s=-1.5 \mathrm{GeV}^{2}$ are purely model effects, as dynamically generated poles are expected to migrate off to infinity as the coupling vanishes.
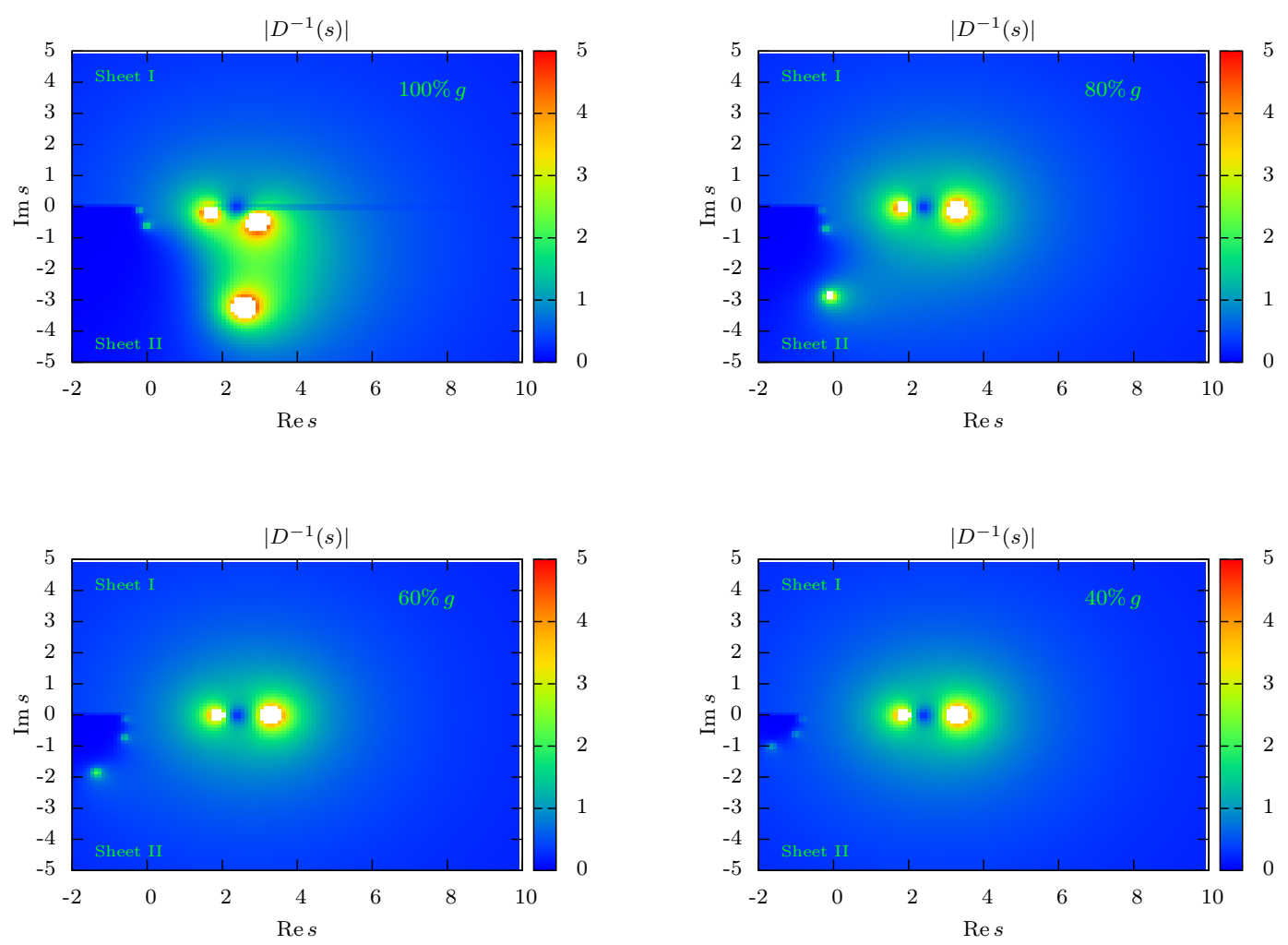

Figure 3.9: The migration of poles as a function of $g$ as $g \rightarrow 0$ in the complex $s^{2}$-plane.

Changing the number of expansion terms between $n_{p}=3$ and $n_{p}=7$ does not in any significant 
way affect the $a_{2}(1320)$ or $a_{2}^{\prime}(1700)$ pole positions. The maximal deviations are $\Delta m\left(a_{2}\right)=5 \mathrm{MeV}$, $\Delta \Gamma\left(a_{2}\right)=7 \mathrm{MeV}$ and $\Delta m\left(a_{2}^{\prime}\right)=40 \mathrm{MeV}, \Delta \Gamma\left(a_{2}^{\prime}\right)=30 \mathrm{MeV}$ between three and seven terms in the $n(s)$ expansion.

In order to demonstrate that coupled-channel effects do not influence the pole positions, we consider an extension of the model to include a second channel also measured by COMPASS, $\rho \pi$ [17, and simultaneously fit the $\eta \pi$ 15 and the $\rho \pi$ [17 final states. The branching ratio of the $a_{2}(1320)$ is saturated at the level of $\sim 85 \%$ by the $\eta \pi$ and $3 \pi$ channels [6], with the $\rho \pi$-wave having the dominant contribution. For simplicity we consider the $\rho$ to be a stable particle with mass $775 \mathrm{MeV}$, the finite width of the $\rho$ being relevant only for $\sqrt{s}<1 \mathrm{GeV}$. The amplitude is then $\hat{\mathcal{A}}_{j}(s)=\sum_{k}\left[\mathcal{D}\left(m_{\eta \pi}\right)\right]_{j k}^{-1}\left(m_{\eta \pi}\right) \mathcal{P}_{k}\left(m_{\eta \pi}\right)$. The denominator is now a $2 \times 2$ matrix, whose diagonal elements are of the form given by Eq. (??), with the appropriate phase space for each channel. The off-diagonal term is parameterized as a single real constant. The production elements $\mathcal{P}_{k}(s)$ are as in Eq. (??), with independent coefficients for each channel. We also performed a $K$-matrix coupled-channel fit and obtained very similar results that are shown in Figure 3.10. The coupledchannel effects produce a competition between the parameters in the numerators to fit the bump at $1.6 \mathrm{GeV}$ in $\eta \pi$ and the dip at $1.8 \mathrm{GeV}$ in $\rho \pi$ at the same time. The $\rho \pi$ data prefers not to have any excited $a_{2}^{\prime}(1700)$, which conversely is evident in the $\eta \pi$ data. Therefore, the uncertainty in the $a_{2}^{\prime}(1700)$ pole position increases, as it is practically unconstrained by the $\rho \pi$ data. Note, however, that in Ref. [17] the dip at $m_{\eta \pi} \sim 1.8 \mathrm{GeV}$ in the $\rho \pi$ data is $t$-dependent, while we use the $t$-integrated intensity, so it may be expected that the effects of the $a_{2}^{\prime}$ are suppressed in our combined fit. We find the following deviations in the pole positions relative to the single-channel fit: $\Delta m\left(a_{2}\right)=2 \mathrm{MeV}, \Delta \Gamma\left(a_{2}\right)=3 \mathrm{MeV}, \Delta m\left(a_{2}^{\prime}\right)=20 \mathrm{MeV}$ and $\Delta \Gamma\left(a_{2}^{\prime}\right)=10 \mathrm{MeV}$. These deviations are rather small and we quote them within our systematic uncertainties. 


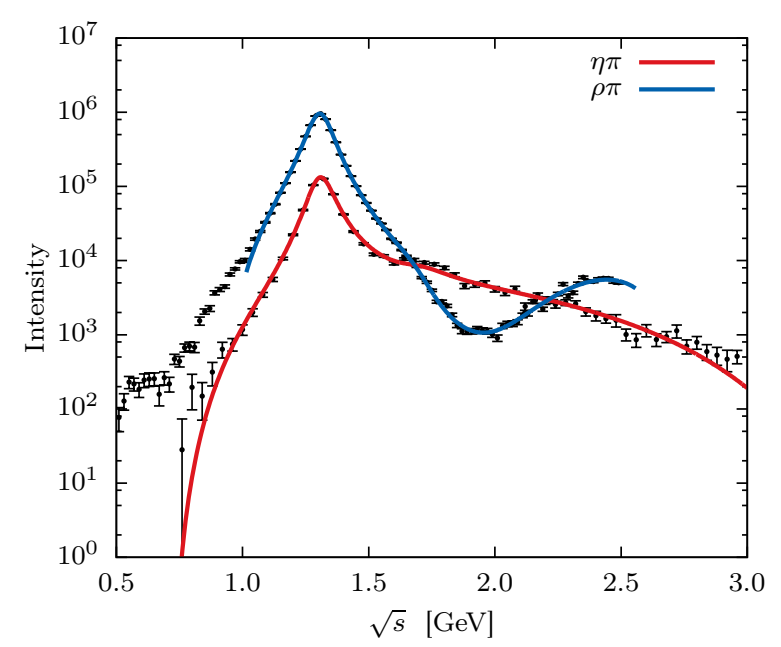

(a) Coupled CDD parameterization

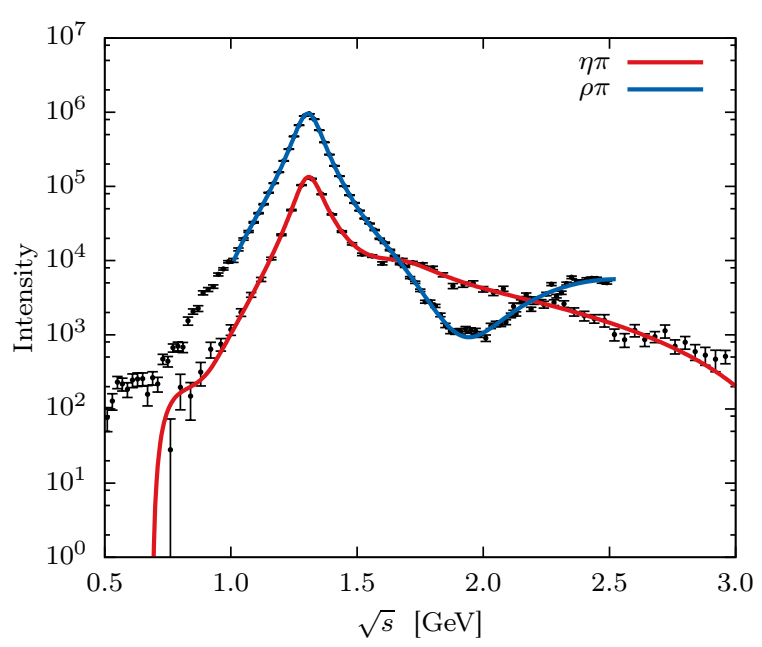

(b) $K$-matrix

Figure 3.10: Coupled-channel $D$-wave fit, (a) using a model based on CDD poles, (b) using the standard $K$-matrix parameterization. Both parameterizations give pole positions consistent with the single-channel analysis. The $\eta \pi$ data is taken from Ref. [15] and the $\rho \pi$ data from Ref. [17].

\subsection{EXOTICA IN $\eta \pi-$ THE $\pi_{1}$ HYBRID CANDIDATE}

Following the successful determination of the $\eta \pi$ tensor resonances, the model was extended to study the coupled channel $\eta \pi$ and $\eta^{\prime} \pi$ system. We focus on applying the coupled channel model on the $P$ - and $D$-waves, to investigate the pole properties of the lightest hybrid candidate, the $\pi_{1}$. The $\eta \pi$ system is a 'Golden mode' for studying hybrid candidates as the spin-exotic $J^{P C}=1^{-+}$ can be directly accessed.

Table 3.2: Resonance parameters. The first error is statistical, the second systematic.

\begin{tabular}{ccc} 
Poles & Mass $(\mathrm{MeV})$ & Width $(\mathrm{MeV})$ \\
\hline$a_{2}(1320)$ & $1306.0 \pm 0.8 \pm 1.3$ & $114.4 \pm 1.6 \pm 0.0$ \\
$a_{2}^{\prime}(1700)$ & $1722 \pm 15 \pm 67$ & $247 \pm 17 \pm 63$ \\
$\pi_{1}$ & $1564 \pm 24 \pm 86$ & $492 \pm 54 \pm 102$
\end{tabular}



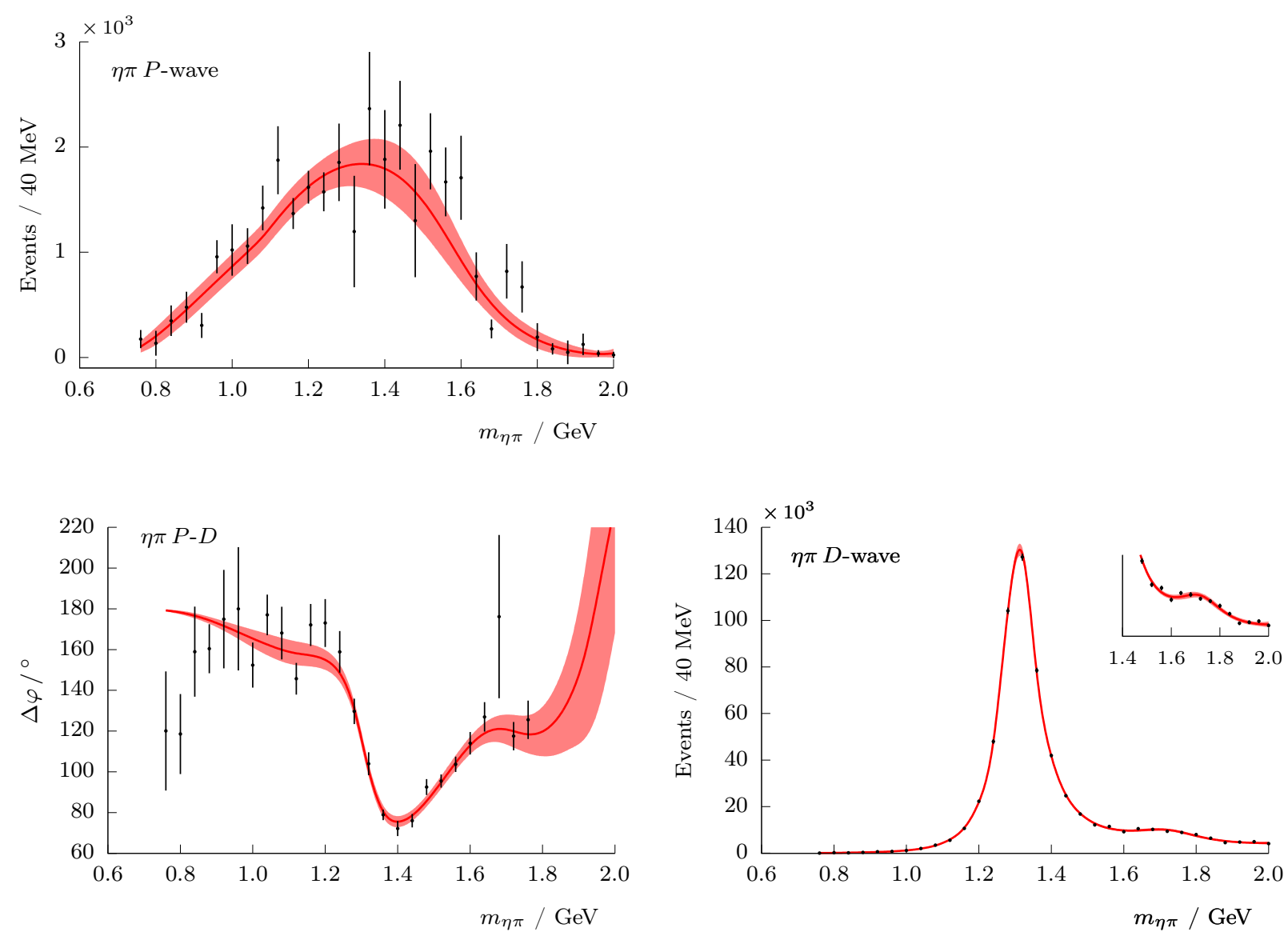

Figure 3.11: Results of the $\eta^{(\prime)} \pi$ coupled-channel fit. Shown are the $\eta \pi P$ - and $D$-wave intensities and their relative phase compared with data from COMPASS [15]. The inset zooms into the region of the $a_{2}^{\prime}(1700)$. The solid line and red band shows the result of the fit and the $2 \sigma$ confidence level provided by the bootstrap analysis, respectively. The initialization of the fit is chosen by randomly generating $O\left(10^{5}\right)$ different sets of values for the parameters. The best fit has $\chi^{2} /$ dof $=162 / 122=$ 1.3. The errors shown are statistical only. Figure adapted from [5]. 

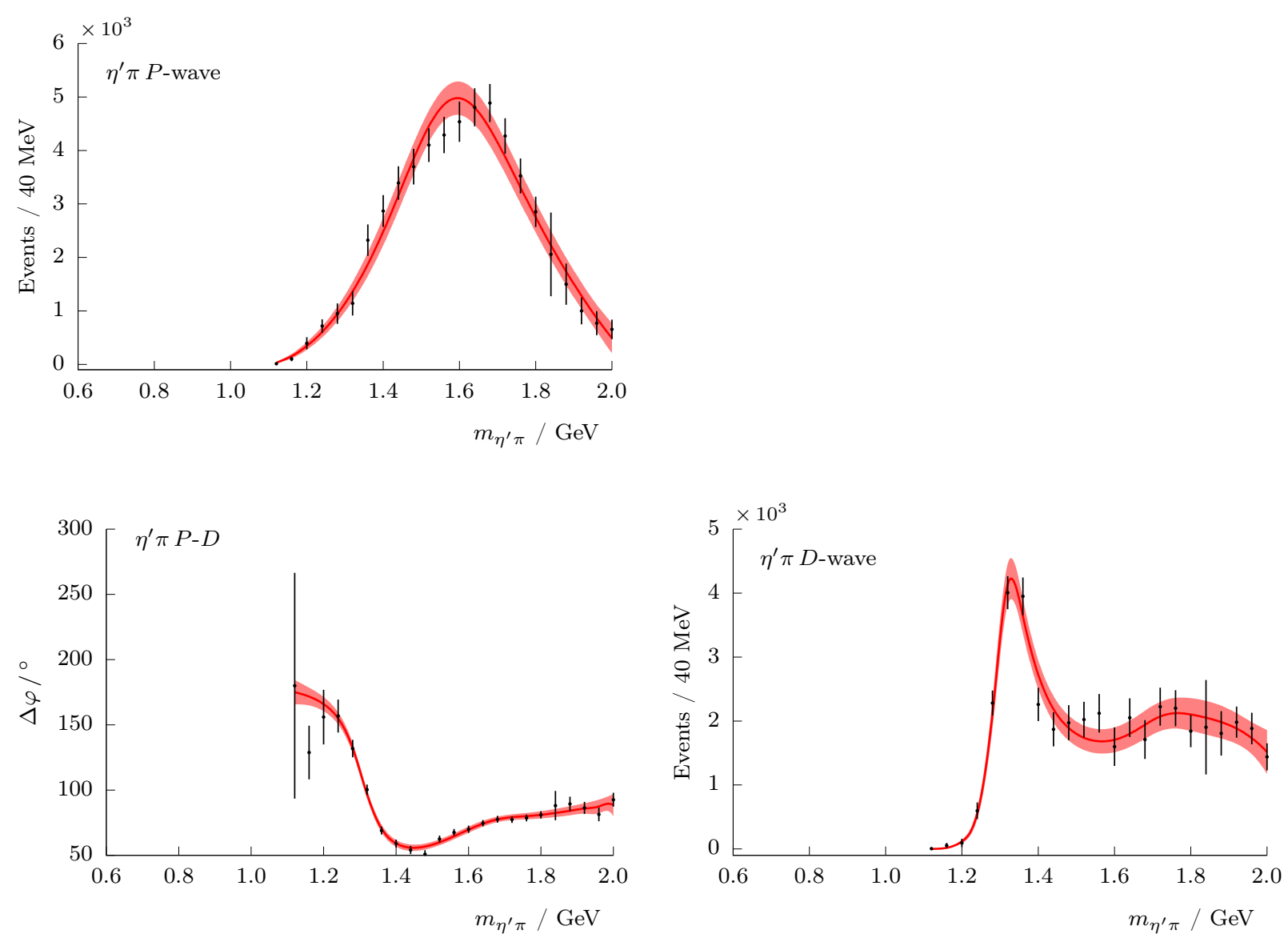

Figure 3.12: Results of the $\eta^{(\prime)} \pi$ coupled-channel fit. Shown are the $\eta^{(\prime)} \pi P$ - and $D$-wave intensities and their relative phase compared with data from COMPASS [15]. The solid line and red band shows the result of the fit and the $2 \sigma$ confidence level provided by the bootstrap analysis, respectively. The initialization of the fit is chosen by randomly generating $O\left(10^{5}\right)$ different sets of values for the parameters. The best fit has $\chi^{2} /$ dof $=162 / 122=1.3$. The errors shown are statistical only. Figure adapted from 5 . 


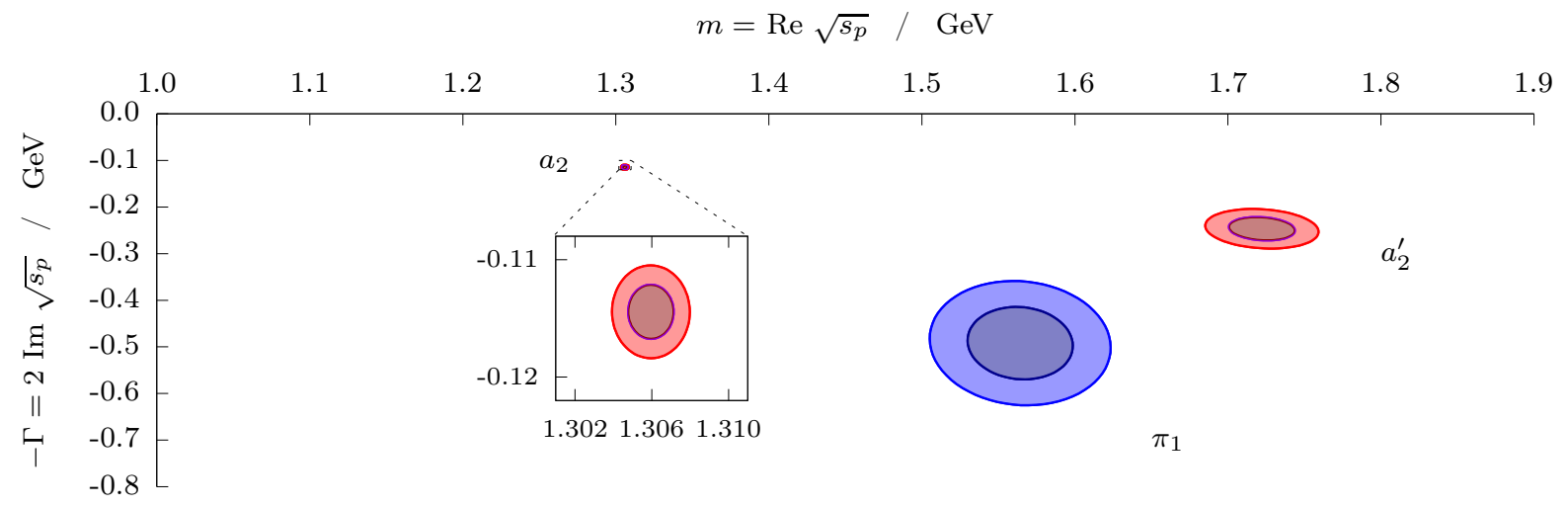

Figure 3.13: Positions of the poles identified as the $a_{2}(1320)$ and $a_{2}^{\prime}(1700)$ (in red) and the $\pi_{1}$ (in blue). The inset shows the position of the $a_{2}(1320)$. The light and dark ellipses show the $1 \sigma$ and $2 \sigma$ confidence levels, respectively. Figure adapted from [5].

All $\eta^{(\prime)} \pi$ waves are measured relative to the $D$-wave, so that we have additional phase information in the form of the phase difference between each wave and the reference $\eta \pi D$-wave. The amplitudes are now expressed via a coupled-channel unitary model

$$
\mathcal{A}_{a}^{J M \epsilon}\left(s, t, E^{\star}\right)=q_{a}^{\star} k^{\star J-1} \sum_{b=1}^{N_{c}} \mathcal{P}_{a}^{J}\left(E^{\star}\right)\left[\mathcal{D}_{J}\left(E^{\star}\right)\right]_{b a}^{-1}
$$

where $E^{\star}$ is the invariant mass of the $\eta^{(\prime)} \pi$ system. The denominator function is the matrix

$$
\left[\mathcal{D}_{J}\left(E^{\star}\right)\right]_{b a}^{-1}=\left[\mathcal{D}_{J}^{(0)}\left(E^{\star}\right)\right]_{b a}^{-1}-\frac{E^{\star 2}}{\pi} \int_{E_{a}^{\star 2}}^{\infty} \mathrm{d} E^{\star / 2} \frac{\rho_{a}^{J}\left(E^{\star \prime}\right) \mathcal{N}_{a b}^{J}\left(E^{\star \prime}\right)}{E^{\star \prime 2}\left(E^{\star / 2}-E^{\star 2}-i \epsilon\right)},
$$

and the production model is the same as the single channel case. The real part is given by the $K$-matrix function

$$
\left[\mathcal{D}_{J}^{(0)}\left(E^{\star}\right)\right]_{b a}^{-1}=\sum_{r} \frac{g_{a}^{J}(r) g_{b}^{J}(r)}{m_{r}^{2}-E^{\star 2}}+c_{a b}^{J}+d_{a b}^{J} E^{\star 2},
$$

where $g_{a}^{J}(r), c_{a b}^{J}=c_{b a}^{J}$, and $d_{a b}^{J}=d_{b a}^{J}$ are real parameters to be determined. Equation (3.45) is a standard parameterization for the $K$-matrix found in literature.

The analysis performed in Ref. [5] showed three resonance poles: two $D$-wave resonances $a_{2}(1320)$ and $a_{2}(1700)$ and single $P$-wave $\pi_{1}$ resonance. Table 3.2 shows the resonance parameters as a result of the coupled channel analysis. Figures 3.11 and 3.12 show the intensity distributions 
with the best fit. Plotted in Fig. 3.13 are the pole positions in the complex $E^{\star 2}$-plane, where the $D$-wave resonances are shown in red and the $P$-wave resonance in blue. The dark and light ellipses are the $1 \sigma$ and $2 \sigma$ error bands, respectively. The $D$-wave parameters are consistent with the single channel analysis, which is consistent with our expectation that poles away from thresholds should be relatively at the same location regardless of the Riemann sheet. The $P$-wave determination is consistent with a single isovector hybrid meson with quantum numbers $J^{P C}=1^{-+}$, as suggested by lattice QCD results 76 78. Its mass and width are determined to be $1564 \pm 24 \pm 86$ and $492 \pm 54 \pm 102 \mathrm{MeV}$, respectively. The statistical uncertainties are estimated via the bootstrap technique, while the systematics due to model dependence are assessed by varying parameters and functional forms that are not directly constrained by unitarity. Solutions with a second exotic $\pi_{1}$ pole were found, however the fit quality was not improved and was found unstable. Therefore, given the COMPASS data, there is only evidence for a single hybrid candidate in $J^{P C}=1^{-+}$.

\subsection{SUMMARY}

Scattering theory provides useful constraints for analysis of hadronic reactions. We applied general unitary models to extract resonance parameters from the $\eta^{(/)} \pi$ system using data from the COMPASS experiment. 


\section{Chapter 4}

\section{PHENOMENOLOGY OF ELASTIC $3 \rightarrow 3$ SCATTERING}

Many of the excited hadrons decay into three particles. Since resonances are poles of scattering amplitudes, hadrons such as the $\omega$ or $a_{1}(1260)$ require the same understanding of $\mathbf{3} \rightarrow \mathbf{3}$ amplitudes as we discussed for the $\mathbf{2} \rightarrow \mathbf{2}$ case. The problem of constraining $\mathbf{3} \rightarrow \mathbf{3}$ reactions from the $S$-matrix principles of unitarity and analyticity has been studied previously in Refs. 79 84. However, with little data, the program ceased progress in understanding the hadron spectrum. In this chapter, we return to the problem of constructing on-shell representations for the elastic scattering of three particles. Our description relies on the isobar approximation, where the amplitude is constructed as a sum of truncated partial wave expansions. This provides a good description of three-particle final states in the resonance region, where analyses of Dalitz plots indicate that they are dominated by intermediate two-body resonances. For example, the decay of the $a_{1}(1260)$ resonance into three pions occurs primarily via a decay to the $\rho \pi$ intermediate state with the subsequent decay of $\rho$ to two pions 17, 18. The isobar approximation can be regarded as an effective way to incorporate the relevant singularities in all Mandelstam variables, and will be discussed in detail later.

\subsection{KINEMATICS, INVARIANTS, \& AMPLITUDES}

We consider elastic scattering of three distinguishable, spinless particle, e.g. $D \pi \bar{D}, K \pi \bar{K}$, or $\pi^{+} \pi^{-} \pi^{0}$. The particles have mass $m_{j}$, where $j=1,2$, or 3 labels the individual particles. A single particle state, with four-momentum $p_{j}=\left(\omega_{j}, \mathbf{p}_{j}\right)$, where $\omega_{j}=\sqrt{m_{j}^{2}+\left|\mathbf{p}_{j}\right|^{2}}$ is the en- 
ergy and $\mathbf{p}_{j}$ is the three-momentum, is denoted $\left|\mathbf{p}_{j}\right\rangle$ and has relativistic normalization $\left\langle\mathbf{p}_{k}^{\prime} \mid \mathbf{p}_{j}\right\rangle=$ $(2 \pi)^{3} 2 \omega_{j} \delta^{(3)}\left(\mathbf{p}_{k}^{\prime}-\mathbf{p}_{j}\right) \delta_{k j}$. We are interested in the $S$-matrix element of the elastic $\mathbf{3} \rightarrow \mathbf{3}$ scattering process. We can decompose the $S$-matrix as $S=\mathbf{1}+i T$. The $T$-matrix contains two terms, $T=T_{d}+T_{c}$, where the disconnected part, $T_{d}$, involves interactions of two particles at the time with the third one being a spectator, while the connected part, $T_{c}$, contains interactions of all three particles. The disconnected part can always be identified kinematically by the spectator momentum conserving delta function 74 . The disconnected part is written as $T_{d}=\sum_{j} \mathbf{1}_{j} \otimes T^{(j)}$, where $\mathbf{1}_{j}$ is the identity operator in the single particle space of the spectator, $j$ and $T^{(j)}$ describes $\mathbf{2} \rightarrow \mathbf{2}$ scattering between the other two particles. The amplitudes associated with the matrix elements of scattering operators $T_{d}$ and $T_{c}$ are defined as $\mathcal{F}$ and $\mathcal{A}$, respectively. Specifically, the connected amplitude $\mathcal{A}$ is given by

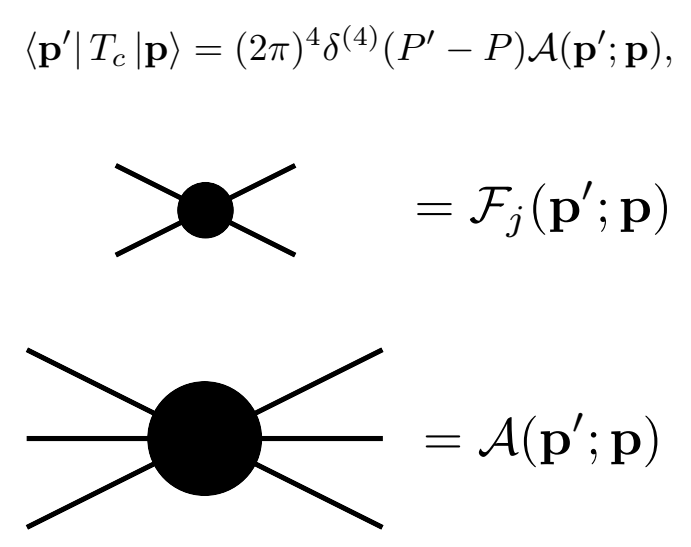

Figure 4.1: Diagrammatic representation of the disconnected $\mathbf{2} \rightarrow \mathbf{2}$ amplitude of Eq. 4.2) (black disk with four external legs) and connected $\mathbf{3} \rightarrow \mathbf{3}$ amplitude of Eq. (4.1) (black disk with six external legs). Figure adapted from Ref. [4].

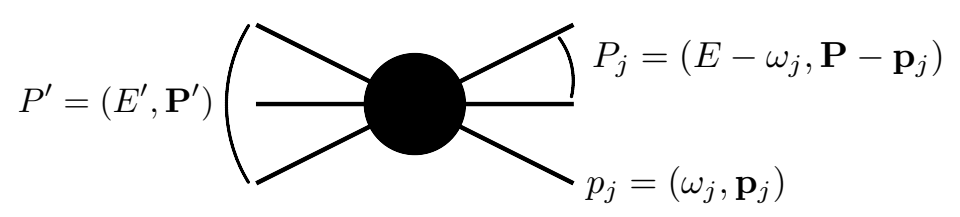

Figure 4.2: Momenta labels for three particles. The left side denotes the final state particles, while the right is the initial state. Figure adapted from Ref. [4]. 
where $|\mathbf{p}\rangle \equiv\left|\mathbf{p}_{1} \mathbf{p}_{2} \mathbf{p}_{3}\right\rangle$ and $\left|\mathbf{p}^{\prime}\right\rangle \equiv\left|\mathbf{p}_{1}^{\prime} \mathbf{p}_{2}^{\prime} \mathbf{p}_{3}^{\prime}\right\rangle$ denote the initial and final states of the three particles, and $P=p_{1}+p_{2}+p_{3}$ and $P^{\prime}=p_{1}^{\prime}+p_{2}^{\prime}+p_{3}^{\prime}$ are the initial and final total four-momenta, respectively, as illustrated in Fig. 4.1. Time-Reversal symmetry implies that the amplitude is symmetric in the initial-final state variables, $\mathcal{A}\left(\mathbf{p}^{\prime} ; \mathbf{p}\right)=\mathcal{A}\left(\mathbf{p} ; \mathbf{p}^{\prime}\right)$. The chosen normalization implies that the amplitude $\mathcal{A}\left(\mathbf{p}^{\prime} ; \mathbf{p}\right)$ has mass dimension -2 . The disconnected amplitudes $\mathcal{F}_{j}$ are defined by

$$
\left\langle\mathbf{p}^{\prime}\left|T_{d}\right| \mathbf{p}\right\rangle=(2 \pi)^{4} \delta^{(4)}\left(P^{\prime}-P\right) \sum_{j=1}^{3}(2 \pi)^{3} 2 \omega_{j} \delta^{(3)}\left(\mathbf{p}_{j}^{\prime}-\mathbf{p}_{j}\right) \mathcal{F}_{j}\left(\mathbf{p}^{\prime} ; \mathbf{p}\right),
$$

where the delta function enforces that the spectator $j$ does not interact. We remark that the $\mathcal{F}_{j}$ is the genuine $\mathbf{2} \rightarrow \mathbf{2}$ scattering amplitude, as required by the LSZ construction [74. We also define $P_{j} \equiv P-p_{j}$ and $P_{j}^{\prime} \equiv P^{\prime}-p_{j}^{\prime}$ as the initial and final total four-momenta of the interacting pair recoiling against spectator $j$, cf. Fig. 4.2 .

We adopt the so-called spectator notation or odd-one-out notation [85], where the $\mathbf{2} \rightarrow \mathbf{2}$ amplitudes associated with the spectator $j$ are labeled by the spectator index. The spectator notation requires additional information specifying the first particle in the two-particle system. There are two conventions which are useful for our discussions: the two-pair convention, and the cyclic convention. The two-pair convention is more practical when interaction in one of the three pairs is negligible. An example of such a system is $\pi^{+} \pi^{+} \pi^{-}$, where the $\pi^{+} \pi^{+}$system interacts weakly. In this case it is convenient to choose the noninteracting system as, say, particles (13) and designate particle 2 as the second particle for both the interacting sub-systems. Therefore, the spectator index $j=1$ and $j=3$ uniquely identifies the two orderings in the pairs to be (32) and (12), respectively. If the interactions in all three subchannels are important, one can define the ordering through cyclical permutation, i.e. the spectator label $j=1,2,3$ corresponds to ordering of the two particles subsystems as (23), (31), and (12), respectively. For simplicity, in the following we assume only two relevant subchannels, and use the former convention. Generalization to the latter case is straightforward. The type of applications we have in mind are systems like $M \bar{M} \pi$ elastic scattering, where $M$ is an open-flavor meson, such as $K, D$, and $B$. The interacting pairs 
will be assumed in the $M \pi$ and $\bar{M} \pi$ channels only, and pion being designated as particle $j=2$.

The $\mathbf{3} \rightarrow \mathbf{3}$ amplitude depends on eight independent kinematic variables. The choice of variables largely depends on the kinematical range of interest, e.g. the low vs high total energy region. Here we are interested in the low-energy region and use the following redundant set of Mandelstam variables,

$$
\begin{aligned}
s & =\left(p_{1}+p_{2}+p_{3}\right)^{2}=\left(p_{1}^{\prime}+p_{2}^{\prime}+p_{3}^{\prime}\right)^{2} \\
t_{j k} & =\left(p_{j}-p_{k}^{\prime}\right)^{2}=\left(P_{j}-P_{k}^{\prime}\right)^{2} \\
u_{j k} & =\left(P_{j}-p_{k}^{\prime}\right)^{2}=\left(\left(P-p_{j}\right)-p_{k}^{\prime}\right)^{2} \\
\sigma_{j} & =P_{j}^{2}=\left(P-p_{j}\right)^{2} \\
\sigma_{k}^{\prime} & =P_{k}^{\prime 2}=\left(P-p_{k}^{\prime}\right)^{2}
\end{aligned}
$$

where $s, \sigma_{j}$, and $\sigma_{k}^{\prime}$ are the invariant mass squares of the total three particle system, the initial pair, and the final pair, respectively. The transferred momenta, $t_{j k}$ and $u_{j k}$, are between the initial and final spectators and the initial pair and final spectator, respectively. The Mandelstam invariants are related by energy-momentum conservation,

$$
\begin{aligned}
s+t_{j k}+u_{j k} & =\sigma_{j}+\sigma_{k}^{\prime}+m_{j}^{2}+m_{k}^{2}, \\
\sum_{j=1}^{3} \sigma_{j} & =s+\sum_{j=1}^{3} m_{j}^{2} \\
\sum_{k=1}^{3} \sigma_{k}^{\prime} & =s+\sum_{k=1}^{3} m_{k}^{2} .
\end{aligned}
$$

In the physical region of the $\mathbf{3} \rightarrow \mathbf{3}$ reaction, $s$ can take any value above the three particle threshold, $s \geq s_{\mathrm{th}}=\left(m_{1}+m_{2}+m_{3}\right)^{2}$, while the subchannel invariant masses $\sigma_{j}$ and $\sigma_{k}^{\prime}$ are bounded by $\sigma_{j}^{(\mathrm{th})} \leq \sigma_{j} \leq\left(\sqrt{s}-m_{j}\right)^{2}$ and $\sigma_{k}^{(\mathrm{th})} \leq \sigma_{k}^{\prime} \leq\left(\sqrt{s}-m_{k}\right)^{2}$, where $\sigma_{j}^{(\mathrm{th})}$ are the sub-energy thresholds, e.g. $\sigma_{1}^{(\mathrm{th})}=\left(m_{2}+m_{3}\right)^{2}$. We will need the relations between the invariants and energies and scattering angles, in three reference frames. The frames of interest will be the overall center-ofmomentum frame $(\mathrm{CMF})$ and the isobar rest frame (IRF). There are two IRFs corresponding to 


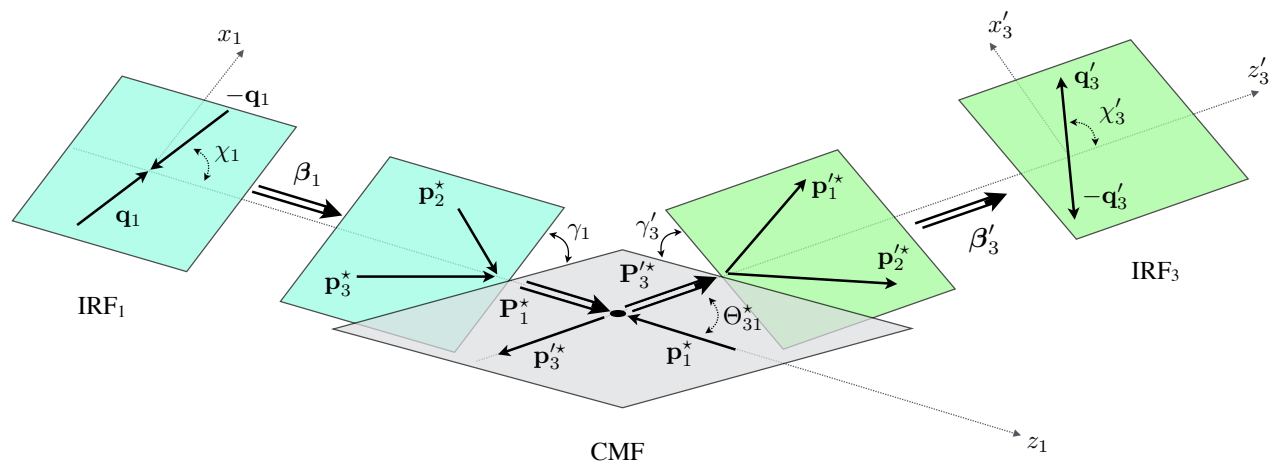

Figure 4.3: Connection between the three reference frames for the $(32) 1 \rightarrow(12) 3$ system. The total reaction plane in the CMF is shown in gray, and the initial and final IRFs are shown in blue and green, respectively. The Lorentz boosts $\boldsymbol{\beta}_{1}$ and $\boldsymbol{\beta}_{3}^{\prime}$ indicate the transformations between the three frames. Figure adapted from Ref. 4].

the initial and final states: the initial $\mathrm{IRF}_{j}$, labeled by the spectator $j$, and the final $\operatorname{IRF}_{k}^{\prime}$, labeled with spectator $k$ and a prime.

To distinguish momenta in the CMF we denote them by a $\star$, i.e. $\mathbf{P}^{\star}=\mathbf{P}^{\star \star}=\mathbf{0}$. In the CMF the scattering angle, $\Theta_{k j}^{\star}$, is defined as the angle between the initial and final state spectator momenta, $\cos \Theta_{k j}^{\star} \equiv \widehat{\mathbf{p}}_{k}^{\prime \star} \cdot \widehat{\mathbf{p}}_{j}^{\star}$, where $\widehat{\mathbf{p}}_{j}^{\star}$ and $\widehat{\mathbf{p}}_{k}^{\prime \star}$ denote the CMF orientations of the initial and final spectators, respectively. The kinematic variables in the other frames, $\operatorname{IRF}_{j}\left(\mathbf{P}_{j}=\mathbf{0}\right)$ and $\operatorname{IRF}_{k}^{\prime}$ $\left(\mathbf{P}_{k}^{\prime}=\mathbf{0}\right)$ are obtained from the CMF by a Lorentz boost in the direction opposite to momentum of the corresponding spectator. The momentum of the first particle in the pair is denoted by $\mathbf{q}_{j}$, and $\mathbf{q}_{k}^{\prime}$ in $\mathrm{IRF}_{j}$ and $\mathrm{IRF}_{k}^{\prime}$, respectively. Orientation of these momenta are given by solid angles, $\widehat{\mathbf{q}}_{j}=\left(\gamma_{j}, \chi_{j}\right)$ and $\widehat{\mathbf{q}}_{k}^{\prime}=\left(\gamma_{k}^{\prime}, \chi_{k}^{\prime}\right)$, respectively. Here, $\gamma_{j}$ and $\gamma_{k}^{\prime}$ are the azimuthal angles between the decay plane of the isobar and the isobar-spectator scattering plane and $\chi_{j}$ and $\chi_{k}^{\prime}$ are helicity angles, cf. Fig. 4.3 for the specific scheme $(23) 1 \rightarrow(12) 3$. The relations between all relevant kinematical variables and the Mandelstam invariants are given in Appendix A. In the following, we will use the set $\left(\widehat{\mathbf{q}}_{k}^{\prime}, \sigma_{k}, s, t_{j k}, \sigma_{j}, \widehat{\mathbf{q}}_{j}\right)$ to describe the isobar-spectator amplitude. 


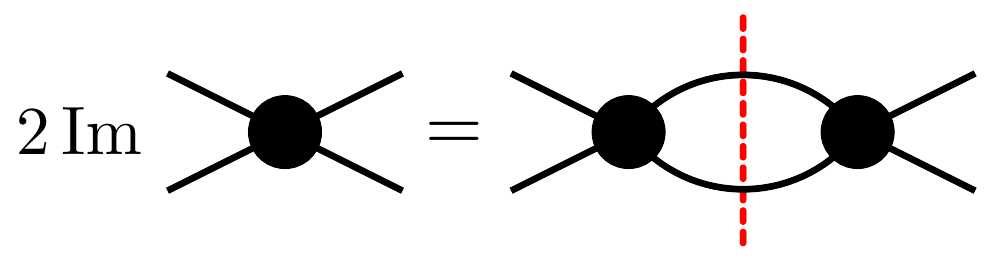

Figure 4.4: Diagrammatic representation for the $\mathbf{2} \rightarrow \mathbf{2}$ disconnected amplitude unitarity relation in Eq. 4.5). The red vertical dashed line indicates the intermediate particles are put on-shell. Figure adapted from Ref. [4].

\subsection{UNITARITY RELATIONS}

We consider elastic unitarity in the physical region of the $\mathbf{3} \rightarrow \mathbf{3}$ reaction below inelastic thresholds. It yields two relations [86], one for the disconnected $\mathbf{2} \rightarrow \mathbf{2}$ amplitude $\mathcal{F}_{j}$ and one for the connected $\mathbf{3} \rightarrow \mathbf{3}$ amplitude $\mathcal{A}$. For $\mathcal{F}_{j}$, one finds

$$
\operatorname{Im} \mathcal{F}_{j}\left(\mathbf{p}^{\prime} ; \mathbf{p}\right)=\rho_{2}\left(\sigma_{j}\right) \int d \widehat{\mathbf{q}}_{j}^{\prime \prime} \mathcal{F}_{j}^{*}\left(\mathbf{p}^{\prime \prime} ; \mathbf{p}^{\prime}\right) \mathcal{F}_{j}\left(\mathbf{p}^{\prime \prime} ; \mathbf{p}\right)
$$

where

$$
\rho_{2}\left(\sigma_{j}\right)=\frac{1}{64 \pi^{2}} \frac{2\left|\mathbf{q}_{j}\right|}{\sqrt{\sigma_{j}}}
$$

is the phase space for the two particle system, and $\mathbf{q}_{j}^{\prime \prime}$ is the intermediate state relative momentum. The IRFs are defined with their $z$-axes defined along the opposite direction of the spectator and their $x$-axes defined by their azimuthal angles w.r.t. the total CMF plane spanned by the initial and final spectator momenta, cf. Fig. 4.3. Note that from energy-momentum conservation, $\left|\mathbf{q}_{j}\right|=$ $\left|\mathbf{q}_{j}^{\prime}\right|=\left|\mathbf{q}_{j}^{\prime \prime}\right|$. Figure 4.4 is a diagrammatic representations of Eq. 4.5. Elastic unitarity yields the following condition for the connected $\mathbf{3} \rightarrow \mathbf{3}$ amplitude, 


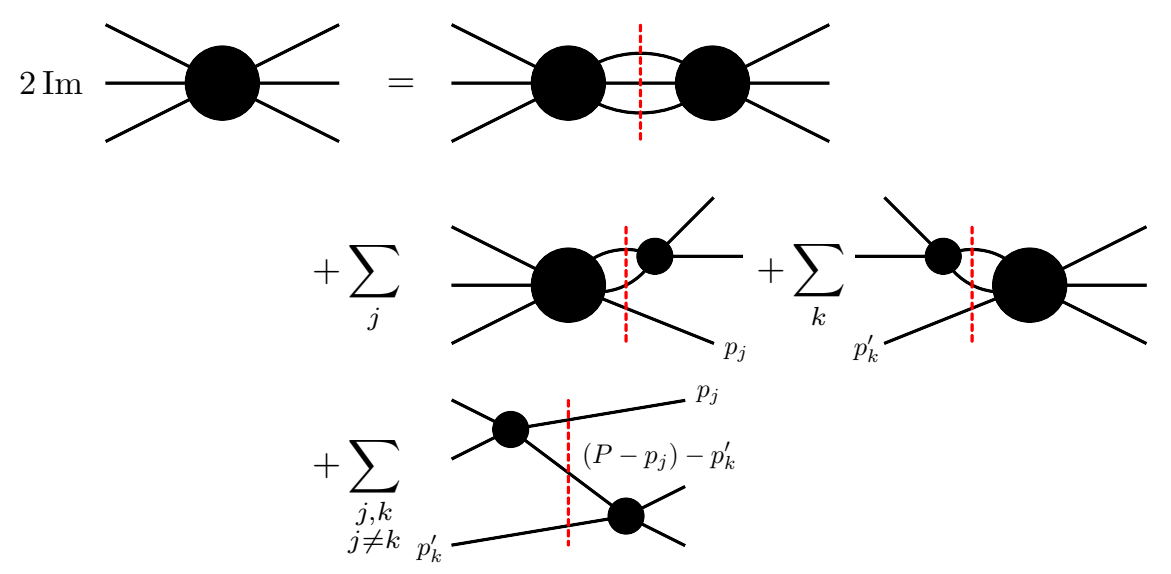

Figure 4.5: Diagrammatic representation for the $\mathbf{3} \rightarrow \mathbf{3}$ connected amplitude unitarity relation in Eq. 4.7). The red vertical dashed line indicates the intermediate particles are put on-shell. Figure adapted from Ref. [4].

$$
\begin{aligned}
\operatorname{Im} \mathcal{A}\left(\mathbf{p}^{\prime} ; \mathbf{p}\right) & =\frac{1}{2(2 \pi)^{5}} \int \frac{d^{3} \mathbf{p}_{1}^{\prime \prime}}{2 \omega_{1}^{\prime \prime}} \frac{d^{3} \mathbf{p}_{2}^{\prime \prime}}{2 \omega_{2}^{\prime \prime}} \frac{d^{3} \mathbf{p}_{3}^{\prime \prime}}{2 \omega_{3}^{\prime \prime}} \delta^{(4)}\left(P^{\prime \prime}-P\right) \mathcal{A}^{*}\left(\mathbf{p}^{\prime \prime} ; \mathbf{p}^{\prime}\right) \mathcal{A}\left(\mathbf{p}^{\prime \prime} ; \mathbf{p}\right) \\
& +\left.\sum_{k} \rho_{2}\left(\sigma_{k}^{\prime}\right) \int d \widehat{\mathbf{q}}_{k}^{\prime \prime} \mathcal{F}_{k}^{*}\left(\mathbf{p}^{\prime \prime} ; \mathbf{p}^{\prime}\right) \mathcal{A}\left(\mathbf{p}^{\prime \prime} ; \mathbf{p}\right)\right|_{\mathbf{p}_{k}^{\prime \prime}=\mathbf{p}_{k}^{\prime}} \Theta\left(\sigma_{k}^{\prime}-\sigma_{k}^{(\mathrm{th})}\right) \\
& +\left.\sum_{j} \rho_{2}\left(\sigma_{j}\right) \int d \widehat{\mathbf{q}}_{j}^{\prime \prime} \mathcal{A}^{*}\left(\mathbf{p}^{\prime \prime} ; \mathbf{p}^{\prime}\right)\right|_{\mathbf{p}_{j}^{\prime \prime}=\mathbf{p}_{j}} \mathcal{F}_{j}\left(\mathbf{p}^{\prime \prime} ; \mathbf{p}\right) \Theta\left(\sigma_{j}-\sigma_{j}^{(\mathrm{th})}\right) \\
& +\left.\left.\sum_{\substack{j, k \\
j \neq k}} \pi \delta\left(u_{j k}-\mu_{j k}^{2}\right) \mathcal{F}_{k}^{*}\left(\mathbf{p}^{\prime \prime} ; \mathbf{p}^{\prime}\right)\right|_{\mathbf{p}_{j}^{\prime \prime}=\mathbf{p}_{j}} \mathcal{F}_{j}\left(\mathbf{p}^{\prime \prime} ; \mathbf{p}\right)\right|_{\mathbf{p}_{k}^{\prime \prime}=\mathbf{p}_{k}^{\prime}},
\end{aligned}
$$

where $\mu_{j k}$ is the mass of the exchanged particle that is neither $j$ nor $k$, e.g. if $j=1$, and $k=3$, then the exchanged mass is $\mu_{13}=m_{2}$. Note that the evaluations $\mathbf{p}_{k}^{\prime \prime}=\mathbf{p}_{k}^{\prime}$ in the second and fourth lines enforce that $\sigma_{k}^{\prime}=\sigma_{k}^{\prime \prime}$, and similarly in lines three and four, $\mathbf{p}_{j}^{\prime \prime}=\mathbf{p}_{j}$ implies that $\sigma_{j}^{\prime \prime}=\sigma_{j}$. Figure 4.5 is a diagrammatic representation of Eq. 4.7) and its derivation is given in Appendix B.

The implications of unitarity for the $\mathcal{F}_{j}$ are summarized below. The unitarity relation for the connected, $\mathcal{A}$ amplitude is more complicated. The first term in Eq. (4.7) is analogous to the $\mathbf{2} \rightarrow \mathbf{2}$ case, in the sense that it is given by the product of the same connected amplitude $\mathcal{A}$. The next two terms originate from the contribution to $S^{\dagger} S$ given by the product of $T_{c}$ and $T_{d}$, and represents the situation when only two of the three particles rescatter. The last term is the contribution to 
the imaginary part of the connected amplitude from the product of two disconnect amplitudes and reflects the real one particle exchange process. Since the unitarity relation deals with physical, on-shell amplitudes, this last contribution is non-vanishing only when the exchanged particle is on-shell, where it is singular and proportional to $\delta\left(u_{j k}-\mu_{j k}^{2}\right)$.

The implications of unitarity for the analytic properties of the $\mathbf{2} \rightarrow \mathbf{2}$ amplitude are well known, and have been discussed in Chapter 2. We review the result here in this notation. In the physical region the partial wave expansion

$$
\mathcal{F}_{j}\left(\mathbf{p}^{\prime} ; \mathbf{p}\right)=\sum_{s_{j}=0}^{\infty} \mathcal{N}_{s_{j}}^{2} f_{s_{j}}\left(\sigma_{j}\right) P_{s_{j}}\left(\widehat{\mathbf{q}}_{j}^{\prime} \cdot \widehat{\mathbf{q}}_{j}\right),
$$

converges and reduces the integral relation given by Eq. (4.5) to a countable set of algebraic ones. Here $s_{j}$ is the angular momentum of the two-particle system $j$ defined in the $\operatorname{IRF}_{j}$-frame, $\mathcal{N}_{s_{j}}^{2}=$ $\left(2 s_{j}+1\right) / 4 \pi$ is a normalization constant, $f_{s_{j}}\left(\sigma_{j}\right)$ is the partial wave amplitude, and $P_{s_{j}}\left(\widehat{\mathbf{q}}_{j}^{\prime} \cdot \widehat{\mathbf{q}}_{j}\right)$ is the Legendre polynomial describing the rotation dependence in terms of the cosine of the $\mathbf{2} \rightarrow \mathbf{2}$ scattering angle. The unitarity relation is diagonalized to the partial wave unitarity relation,

$$
\operatorname{Im} f_{s_{j}}\left(\sigma_{j}\right)=\rho_{2}\left(\sigma_{j}\right)\left|f_{s_{j}}\left(\sigma_{j}\right)\right|^{2} \Theta\left(\sigma_{j}-\sigma_{j}^{\text {(th) }}\right) .
$$

This equation is automatically satisfied by

$$
f_{s_{j}}^{-1}\left(\sigma_{j}\right)=K_{s_{j}}^{-1}\left(\sigma_{j}\right)-\frac{1}{\pi} \int_{\sigma_{j}^{\text {(th) }}}^{\infty} d \widehat{\sigma} \frac{\rho_{2}(\widehat{\sigma})}{\widehat{\sigma}-\sigma_{j}}
$$

where the $K$-matrix is a real function along the unitarity cut.

The $\mathbf{3} \rightarrow \mathbf{3}$ amplitude in the physical region can be expanded in partial waves in any of the (12), (13), (23) subsystems. We refer to a subchannel of choice, e.g. (12) as the direct channel and to the others as the cross channels. Since each term in the partial wave expansion is analytic in the angular variables, and therefore in the (13) and (23) invariant masses, singularities in the latter variables can happen only when the series diverges. In contrast to the $\mathbf{2} \rightarrow \mathbf{2}$ case, the unitarity equations for each partial wave would not decouple, and would contain an infinite number of terms. Since in practice one must truncate the series, the amplitude would be regular in the (13) and (23) 
invariant masses, and the information about the cross channels dynamics would be lost. Instead, we will represent $\mathbf{3} \rightarrow \mathbf{3}$ amplitude in an isobar approximation, where only a finite number of terms in the direct and cross channels are included.

\subsection{THE ISOBAR REPRESENTATION}

To be concrete, the partial wave expansion of the connected $\mathbf{3} \rightarrow \mathbf{3}$ amplitude reads

$$
\mathcal{A}\left(\mathbf{p}^{\prime} ; \mathbf{p}\right)=\sum_{J} \sum_{\ell_{k}^{\prime}, s_{k}^{\prime}} \sum_{\ell_{j}, s_{j}} \mathcal{M}_{\ell_{k}^{\prime} s_{k}^{\prime} ; \ell_{j} s_{j}}^{J}\left(\sigma_{k}^{\prime}, s, \sigma_{j}\right) \sum_{M} Z_{\ell_{k}^{\prime} s_{k}^{\prime}}^{J M} *\left(\widehat{\mathbf{P}}_{k}^{\prime \star}, \widehat{\mathbf{q}}_{k}^{\prime}\right) Z_{\ell_{j} s_{j}}^{J M}\left(\widehat{\mathbf{P}}_{j}^{\star}, \widehat{\mathbf{q}}_{j}\right),
$$

where we project the amplitude onto the chosen $j$ and $k$ initial and final channels. Here $s_{j}\left(s_{k}^{\prime}\right)$ is the angular momentum of the initial (final) pair, $\ell_{j}\left(\ell_{k}^{\prime}\right)$ is the angular momentum between the pair and the spectator, $J$ and $M$ are the total angular momentum of the three particles and its projection, and $\mathcal{M}_{\ell_{k}^{\prime} s_{k}^{\prime} ; \ell_{j} s_{j}}^{J}$ is the partial wave amplitude. The angles $\widehat{\mathbf{P}}_{j}^{\star}$ and $\widehat{\mathbf{P}}_{k}^{\prime \star}$ are the orientations of the initial and final pair, which are related to the CMF scattering angle via $\cos \Theta_{k j}^{\star}=\widehat{\mathbf{P}}_{k}^{\prime \star} \cdot \widehat{\mathbf{P}}_{j}^{\star}$. The functions $Z_{\ell s}^{J M}$ contain the rotational dependence of the amplitude $\mathcal{A}$, which are defined as

$$
Z_{\ell s}^{J M}(\widehat{\mathbf{P}}, \widehat{\mathbf{q}})=\mathcal{N}_{\ell} \mathcal{N}_{s} \sum_{\lambda=-s}^{s}\langle J \lambda \mid \ell 0 s \lambda\rangle \mathcal{D}_{M \lambda}^{(J)}(\widehat{\mathbf{P}}) \mathcal{D}_{\lambda 0}^{(s)}(\widehat{\mathbf{q}})
$$

The $Z$-functions contain all the angular dependence, and they fulfill the orthonormality condition

$$
\int d \widehat{\mathbf{P}} \int d \widehat{\mathbf{q}} Z_{\ell^{\prime} s^{\prime}}^{J^{\prime} M^{\prime} *}(\widehat{\mathbf{P}}, \widehat{\mathbf{q}}) Z_{\ell s}^{J M}(\widehat{\mathbf{P}}, \widehat{\mathbf{q}})=\delta_{J J^{\prime}} \delta_{M M^{\prime}} \delta_{\ell \ell^{\prime}} \delta_{s s^{\prime}}
$$

More details are in Appendix C.

We next discuss the relation between partial wave expansion, isobar representation, and finally the isobar approximation. The partial wave expansion given by Eq. 4.11) is in principle an exact representation of the amplitude in the physical region of $\mathbf{3} \rightarrow \mathbf{3}$ scattering. However, unlike the analogous expansion in $\mathbf{2} \rightarrow \mathbf{2}$ scattering, the partial wave expansion cannot be used in practice in the $\mathbf{3} \rightarrow \mathbf{3}$ case.

In practice, one needs to restrict the series to a finite number of partial waves. In the physical region of $\mathbf{2} \rightarrow \mathbf{2}$ scattering, the low-energy behavior of the partial waves is determined by barrier 
factors due to the finite range of interactions. This suppresses the strength of higher partial waves at threshold, provided the latter are regular in the cross channel Mandelstam variables. Cross channel exchanges generate singularities that spoil the convergence of the partial wave series. However, in the $\mathbf{2} \rightarrow \mathbf{2}$ kinematics, these singularities do not overlap with the direct channel physical region. Therefore, the partial wave series can be safely truncated in a finite domain of CMF energies above the two particle threshold.

This is not the case, for example, when one of the particles can decay to the other three, and similarly it is never the case for $\mathbf{3} \rightarrow \mathbf{3}$ scattering. If we consider indistinguishable particles, explicit Bose symmetry is lost for the $\mathcal{M}_{\ell_{k}^{\prime} s_{k}^{\prime} ; \ell_{j} s_{j}}^{J}$ partial waves, since the partial wave expansion in the initial and final states singles out specific two-body channels. The symmetry is only recovered upon resummation. The isobar representation, in principle, takes care of this problem. One writes the connected $\mathbf{3} \rightarrow \mathbf{3}$ amplitude as a redundant sum of expansions in all the initial and final pairs to make the symmetry explicit. Bose symmetry is thus preserved upon truncation.

As discussed above, one can manage only a finite number of terms in the sums over the subchannel spins. Therefore one reduces the isobar representation

$$
\mathcal{A}\left(\mathbf{p}^{\prime} ; \mathbf{p}\right)=\sum_{j, k} \mathcal{A}_{k j}\left(\mathbf{p}^{\prime} ; \mathbf{p}\right),
$$

to the isobar approximation, by representing the connected $\mathbf{3} \rightarrow \mathbf{3}$ amplitude as a sum over a finite number of isobar-spectator amplitudes,

$$
\mathcal{A}_{k j}\left(\mathbf{p}^{\prime} ; \mathbf{p}\right)=\sum_{J} \sum_{\ell_{k}^{\prime}, s_{k}^{\prime}}^{\max ^{\prime}} \sum_{\ell_{j}, s_{j}}^{\max } \mathcal{A}_{\ell_{k}^{\prime} s_{k}^{\prime} ; \ell_{j} s_{j}}^{J}\left(\sigma_{k}^{\prime}, s, \sigma_{j}\right) \sum_{M} Z_{\ell_{k}^{\prime} s_{k}^{\prime}}^{J M} *\left(\widehat{\mathbf{P}}_{k}^{\prime \star}, \widehat{\mathbf{q}}_{k}^{\prime}\right) Z_{\ell_{j} s_{j}}^{J M}\left(\widehat{\mathbf{P}}_{j}^{\star}, \widehat{\mathbf{q}}_{j}\right),
$$

as shown in Fig. 4.6. The truncation is reflected by "max" in the sums. We projected the isobarspectator amplitudes onto the total angular momentum $J$ of the three particle system. In the following, we refer to $\mathcal{A}_{\ell_{k}^{\prime} s_{k}^{\prime} ; \ell_{j} s_{j}}^{J}\left(\sigma_{k}^{\prime}, s, \sigma_{j}\right)$ as the partial wave isobar spectator (PWIS) amplitudes. We emphasize that, while truncation in $s_{k}^{\prime}$ and $s_{j}$ cannot be avoided in practice, unitarity is diagonal in the total angular momentum. Amplitudes for each $J$ are thus independent and can in principle be resummed. 


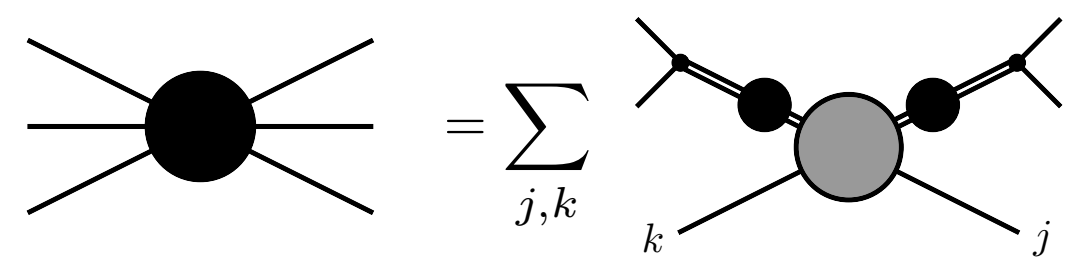

Figure 4.6: Diagrammatic representation of the isobar approximation amplitude in Eq. (4.14). The double lines with the black disk represents the isobar amplitude $f_{s_{j}}\left(\sigma_{j}\right)$, while the gray disk represents the isobar-spectator amplitude $\mathcal{A}_{k j}\left(\mathbf{p}^{\prime} ; \mathbf{p}\right)$. Figure adapted from Ref. [4.

We also stress that the PWIS amplitudes $\mathcal{A}_{\ell_{k}^{\prime} s_{k}^{\prime} ; \ell_{j} s_{j}}^{J}$ are not the genuine $\mathbf{3} \rightarrow \mathbf{3}$ partial wave amplitudes $\mathcal{M}_{\ell_{k}^{\prime} s_{k}^{\prime} ; \ell_{j} s_{j}}^{J}$ in Eq. 4.11:

$$
\mathcal{M}_{\ell_{k}^{\prime} s_{k}^{\prime} ; \ell_{j} s_{j}}^{J}\left(\sigma_{k}^{\prime}, s, \sigma_{j}\right)=\mathcal{A}_{\ell_{k}^{\prime} s_{k}^{\prime} ; \ell_{j} s_{j}}^{J}\left(\sigma_{k}^{\prime}, s, \sigma_{j}\right)+X_{\ell_{k}^{\prime} s_{k}^{\prime} ; \ell_{j} s_{j}}^{J}\left(\sigma_{k}^{\prime}, s, \sigma_{j}\right)
$$

where $X_{\ell_{k}^{\prime} s_{k}^{\prime} ; \ell_{j} s_{j}}^{J}$ contains all the cross channel terms which recouple to the direct channel amplitude,

$$
\begin{aligned}
X_{\ell_{k}^{\prime} s_{k}^{\prime} ; \ell_{j} s_{j}}^{J}\left(\sigma_{k}^{\prime}, s, \sigma_{j}\right) & =\sum_{\substack{a \neq j, b \neq k}} \sum_{\ell_{b}^{\prime}, s_{b}^{\prime}} \sum_{\ell_{a}, s_{a}} \int d \widehat{\mathbf{P}}_{k}^{\prime \star} \int d \widehat{\mathbf{q}}_{k}^{\prime} \int d \widehat{\mathbf{P}}_{j}^{\star} \int d \widehat{\mathbf{q}}_{j} \mathcal{A}_{\ell_{b}^{\prime} s_{b}^{\prime} ; \ell_{a} s_{a}}^{J}\left(\sigma_{b}^{\prime}, s, \sigma_{a}\right) \\
& \times Z_{\ell_{k}^{\prime} s_{k}^{\prime}}^{J M}\left(\widehat{\mathbf{P}}_{k}^{\prime \star}, \widehat{\mathbf{q}}_{k}^{\prime}\right) Z_{\ell_{b}^{\prime} s_{b}^{\prime}}^{J M}\left(\widehat{\mathbf{P}}_{b}^{\prime \star}, \widehat{\mathbf{q}}_{b}^{\prime}\right) Z_{\ell_{j} s_{j}}^{J M *}\left(\widehat{\mathbf{P}}_{j}^{\star}, \widehat{\mathbf{q}}_{j}\right) Z_{\ell_{a} s_{a}}^{J M}\left(\widehat{\mathbf{P}}_{a}^{\star}, \widehat{\mathbf{q}}_{a}\right) .
\end{aligned}
$$

The kinematic relations given in Appendix A can be used to write the cross channel variables in terms of the direct channel variables.

Often in the literature, Bose symmetry is considered as a motivation for Eq. 4.14. However, this is completely independent: the representation can be applied to the distinguishable particle case (in this case the various $\mathcal{A}_{k j}\left(\mathbf{p}^{\prime} ; \mathbf{p}\right)$ contain different physics and have different functional forms), and Bose symmetry can be imposed to the expansion in Eq. 4.11 without requiring an explicit sum over channels. For example we consider the $\pi^{+} \pi^{-} \pi^{0} \rightarrow \pi^{+} \pi^{-} \pi^{0}$ process in the isoscalar vector channel, where the $\omega$ is observed. Thinking in isospin basis, where the three pions are indistinguishable, and in the charge basis, where they are distinguishable, leads to the same form of the amplitude, showing that Bose symmetry plays no role in defining the representation.

Isobars parameterize the $\mathbf{2} \rightarrow \mathbf{2}$ dynamics in a given subchannel and angular momentum 
state. Contrary to the $\mathbf{2} \rightarrow \mathbf{2}$ partial waves, they have only right hand singularities constrained by unitarity. The isobars can have resonant or bound state poles. In the $N / D$ formalism, the isobars can be identified with the denominator function, where the left hand cuts are removed via a dispersive integral [52]. In the following, we will ignore all left hand singularities of the $\mathbf{2} \rightarrow \mathbf{2}$ amplitudes, and identify their partial waves with the isobars. Although we do not need to assume any resonant content for the isobars (e.g. we could use an isobar to describe the $\pi^{+} \pi^{+}$dynamics), it is a popular picture to think of them as a quasi-particle, and to identify the invariant mass and angular momentum of the pair with the isobar mass and spin. Isobars are customarily labeled with the name of the dominant resonance, if any. Isobars can be parameterized as in Eq. 4.10). For example, the $a_{1}(1260)$ decays into three pions dominantly in the $\rho \pi$ and $\sigma \pi$ channels 87 . If one chooses to perform a truncated partial wave expansion of the $\mathbf{3} \rightarrow \mathbf{3}$ amplitude in only the $\rho \pi \rightarrow a_{1}(1260) \rightarrow \rho \pi$ channel, rescattering effects between the $\rho \pi \rightarrow \sigma \pi$ isobars are ignored. The isobar approximation corrects this by including amplitudes for $\sigma \pi \rightarrow a_{1}(1260) \rightarrow \rho \pi$, $\rho \pi \rightarrow a_{1}(1260) \rightarrow \sigma \pi$, and $\sigma \pi \rightarrow a_{1}(1260) \rightarrow \sigma \pi$.

The approximation is expected to be valid at low values of energy, where a finite number of singularities dominate the amplitude. Moving to higher energies, the left hand cuts controlling the crossed $\mathbf{2} \rightarrow \mathbf{4}$ processes will become relevant, and the behavior of the amplitude will be controlled by analyticity in angular momentum, rather than direct-channel unitarity.

Since the isobar approximation includes the cross channel effects in the summation, the isobarspectator amplitudes contain only normal threshold singularities determined by unitarity. Therefore, the analytic structure of each isobar-spectator amplitude in the energy variables, $s, \sigma_{j}$, and $\sigma_{k}^{\prime}$, are determined by unitarity.

The problem of convergence in $J$ is more severe. The $\mathbf{3} \rightarrow \mathbf{3}$ amplitude contains an OPE process (see the last diagram in Fig. 4.5, which can go on-shell in the direct channel, and results in an interaction of infinite range. In this case the cross channel singularities overlap with the physical region and project onto an infinite number of partial waves. The analytic properties of 
the projected amplitude are highly nontrivial. We discuss them in detail in Section 5.2 . However, since the main goal in this and similar studies of three particle scattering is to identify the spectrum, ultimately one needs to deal with amplitudes of well defined total angular momentum $J$. In other words, these amplitudes diagonalize unitarity, which is the basis for analytic continuation and identification of complex singularities as resonance poles. For this reason, in the following we will not address the problem of convergence in $J$.

\subsubsection{PWIS UNITARITY RELATIONS}

It is advantageous to introduce an amputated PWIS amplitude $\widetilde{\mathcal{A}}_{\ell_{k}^{\prime} s_{k}^{\prime} ; \ell_{j} s_{j}}^{J}$, in which the isobar amplitudes are factorized,

$$
\mathcal{A}_{\ell_{k}^{\prime} s_{k}^{\prime} ; \ell_{j} s_{j}}^{J}=f_{s_{k}^{\prime}}\left(\sigma_{k}^{\prime}\right) \widetilde{\mathcal{A}}_{\ell_{k}^{\prime} s_{k}^{\prime} ; \ell_{j} s_{j}}^{J}\left(\sigma_{k}^{\prime}, s, \sigma_{j}\right) f_{s_{j}}\left(\sigma_{j}\right)
$$

The amputation reduces the number of terms in the isobar-spectator unitarity relation by making use of subchannel unitarity in Eq. 4.9. However, the amputated PWIS amplitudes still have a non-trivial dependence on the subchannel energies due to rescattering effects. As shown in detail in Appendix C, combining Eqs. 4.7), 4.9), 4.12, 4.13, 4.15), and 4.18 results in the amputated PWIS unitarity relation 


$$
\begin{aligned}
& \operatorname{Im} \widetilde{\mathcal{A}}_{\ell_{k}^{\prime} s_{k}^{\prime} ; \ell_{j} s_{j}}^{J}\left(\sigma_{k}^{\prime}, s, \sigma_{j}\right) \\
& =\frac{1}{\pi\left(32 \pi^{2}\right)^{2}} \sum_{n} \sum_{\ell_{n}^{\prime \prime}, s_{n}^{\prime \prime}} \int_{\sigma_{n}^{(\mathrm{th})}}^{\left(\sqrt{s}-m_{n}\right)^{2}} d \sigma_{n}^{\prime \prime} \frac{\left|\mathbf{q}_{n}^{\prime \prime}\right|\left|\mathbf{p}_{n}^{\prime \prime \star}\right|}{\sqrt{\sigma_{n}^{\prime \prime} \sqrt{s}}}\left|f_{s_{n}^{\prime \prime}}\left(\sigma_{n}^{\prime \prime}\right)\right|^{2} \\
& \times \widetilde{\mathcal{A}}_{\ell_{n}^{\prime \prime} s_{n}^{\prime \prime} ; \ell_{k}^{\prime} s_{k}^{\prime}}^{*}\left(\sigma_{n}^{\prime \prime}, s, \sigma_{k}^{\prime}\right) \widetilde{\mathcal{A}}_{\ell_{n}^{\prime \prime} s_{n}^{\prime \prime} ; \ell_{j} s_{j}}^{J}\left(\sigma_{n}^{\prime \prime}, s, \sigma_{j}\right) \Theta\left(s-s_{\mathrm{th}}\right) \\
& +\frac{1}{2 \pi s\left(32 \pi^{2}\right)^{2}} \sum_{\substack{n, r \\
n \neq r}} \sum_{\ell_{n}^{\prime \prime}, s_{n}^{\prime \prime}} \sum_{\ell_{r}^{\prime \prime}, s_{r}^{\prime \prime}} \int_{\sigma_{n}^{(\mathrm{th})}}^{\left(\sqrt{s}-m_{n}\right)^{2}} d \sigma_{n}^{\prime \prime} \int_{\sigma_{r}^{(-)}\left(\sigma_{n}^{\prime \prime}\right)}^{\sigma_{r}^{(+)}\left(\sigma_{n}^{\prime \prime}\right)} d \sigma_{r}^{\prime \prime} f_{s_{r}^{\prime \prime}}^{*}\left(\sigma_{r}^{\prime \prime}\right) f_{s_{n}^{\prime \prime}}\left(\sigma_{n}^{\prime \prime}\right) \\
& \times \mathcal{C}_{\ell_{n}^{\prime \prime} s_{n}^{\prime \prime} ; \ell_{r}^{\prime \prime} s_{r}^{\prime \prime}}^{J}\left(\sigma_{n}^{\prime \prime}, s, \sigma_{r}^{\prime \prime}\right) \widetilde{\mathcal{A}}_{\ell_{r}^{\prime \prime} s_{r}^{\prime \prime} ; \ell_{k}^{\prime} s_{k}^{\prime}}^{J *}\left(\sigma_{r}^{\prime \prime}, s, \sigma_{k}^{\prime}\right) \widetilde{\mathcal{A}}_{\ell_{n}^{\prime \prime} s_{n}^{\prime \prime} ; \ell_{j} s_{j}}^{J}\left(\sigma_{n}^{\prime \prime}, s, \sigma_{j}\right) \Theta\left(s-s_{\mathrm{th}}\right) \\
& +\frac{1}{64 \pi^{2} \sqrt{s}} \frac{1}{\left|\mathbf{p}_{k}^{\prime \star}\right|} \sum_{r \neq k} \sum_{\ell_{r}^{\prime \prime}, s_{r}^{\prime \prime}} \int_{\sigma_{r}^{(\mathrm{th})}}^{\left(\sqrt{s}-m_{r}\right)^{2}} d \sigma_{r}^{\prime \prime} \mathcal{C}_{\ell_{k}^{\prime} s_{k}^{\prime} ; \ell_{r}^{\prime \prime} s_{r}^{\prime \prime}}^{J}\left(\sigma_{k}^{\prime}, s, \sigma_{r}^{\prime \prime}\right) \\
& \times f_{s_{r}^{\prime \prime}}\left(\sigma_{r}^{\prime \prime}\right) \widetilde{\mathcal{A}}_{\ell_{r}^{\prime \prime} s_{r}^{\prime \prime} ; \ell_{j} s_{j}}^{J}\left(\sigma_{r}^{\prime \prime}, s, \sigma_{j}\right) \Theta\left(\sigma_{k}^{\prime}-\sigma_{k}^{(\mathrm{th})}\right) \\
& +\frac{1}{64 \pi^{2} \sqrt{s}} \frac{1}{\left|\mathbf{p}_{j}^{\star}\right|} \sum_{n \neq j} \sum_{\ell_{n}^{\prime \prime}, s_{n}^{\prime \prime}} \int_{\sigma_{n}^{(\text {th })}}^{\left(\sqrt{s}-m_{n}\right)^{2}} d \sigma_{n}^{\prime \prime} \mathcal{C}_{\ell_{n}^{\prime \prime} s_{n}^{\prime \prime} ; \ell_{j} s_{j}}^{J}\left(\sigma_{n}^{\prime \prime}, s, \sigma_{j}\right) \\
& \times f_{s_{n}^{\prime \prime}}^{*}\left(\sigma_{n}^{\prime \prime}\right) \widetilde{\mathcal{A}}_{\ell_{k}^{\prime} s_{k}^{\prime} ; \ell_{n}^{\prime \prime} s_{n}^{\prime \prime}}^{J *}\left(\sigma_{k}^{\prime}, s, \sigma_{n}^{\prime \prime}\right) \Theta\left(\sigma_{j}-\sigma_{j}^{(\mathrm{th})}\right) \\
& +\frac{\pi}{2\left|\mathbf{p}_{j}^{\star}\right|\left|\mathbf{p}_{k}^{\prime \star}\right|} \mathcal{C}_{\ell_{k}^{\prime} s_{k}^{\prime} ; \ell_{j} s_{j}}^{J}\left(\sigma_{k}^{\prime}, s, \sigma_{j}\right)\left(1-\delta_{j k}\right) \Theta\left(1-\cos ^{2} \theta_{k j}^{\star}\right),
\end{aligned}
$$

where $\mathcal{C}_{\ell_{n}^{\prime \prime} s_{n}^{\prime \prime} ; \ell_{r}^{\prime \prime} s_{r}^{\prime \prime}}^{J}\left(\sigma_{n}^{\prime \prime}, s, \sigma_{r}^{\prime \prime}\right)$ is a purely kinematical recoupling coefficient between different intermediate state isobars,

$$
\begin{aligned}
\mathcal{C}_{\ell_{k} s_{k} ; \ell_{j} s_{j}}^{J}\left(\sigma_{k}, s, \sigma_{j}\right) & =2 \pi \mathcal{N}_{s_{j}} \mathcal{N}_{s_{k}} \mathcal{N}_{\ell_{j}} \mathcal{N}_{\ell_{k}} \mathcal{N}_{J}^{-2} \sum_{\lambda_{k}, \lambda_{k}}\left\langle J \lambda_{k} \mid \ell_{k} 0 s_{k} \lambda_{k}\right\rangle\left\langle J \lambda_{j} \mid \ell_{j} 0 s_{j} \lambda_{j}\right\rangle \\
& \times d_{\lambda_{k} 0}^{\left(s_{k}\right)}\left(\cos \chi_{k}\right) d_{\lambda_{k} \lambda_{j}}^{(J)}\left(\cos \theta_{k j}^{\star}\right) d_{\lambda_{j} 0}^{\left(s_{j}\right)}\left(\cos \chi_{j}\right) .
\end{aligned}
$$

The recoupling coefficients relate two different orientations of three particles in the same frame 83, 85, 88. Appendix $\mathrm{C}$ contains details on the derivation of the recoupling coefficients from the rotational matrices in Eq. 4.12). The helicity angles and the CMF angle between particles $j$ and $k, \theta_{k j}^{\star}$, are functions of the invariants (cf. Appendix A).

The second term contains two integrals over the Dalitz region of the three-particles in the intermediate state, where the physical region is bounded by $\sigma_{n}^{(\mathrm{th})} \leq \sigma_{n}^{\prime \prime} \leq\left(\sqrt{s}-m_{n}\right)^{2}$ and $\sigma_{r}^{(-)} \leq$ $\sigma_{r}^{\prime \prime} \leq \sigma_{r}^{(+)}$, where $\sigma_{r}^{( \pm)}$is a function of $\sigma_{n}^{\prime \prime}$ and gives the physical boundary $\cos \chi_{n}^{\prime \prime}= \pm 1$, e.g. for 


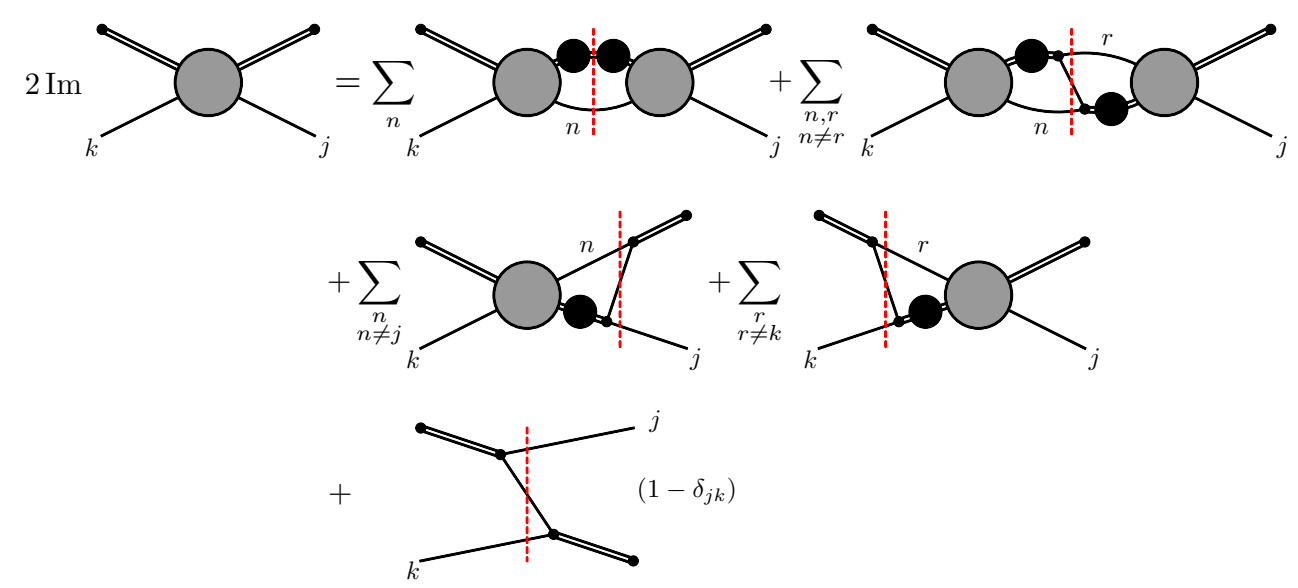

Figure 4.7: Diagrammatic representation for the amputated PWIS unitarity relation in Eq. 4.19. The black disks in the internal legs represent the isobars, which are amputated from the external legs, see Eq. 4.18). The cuts across the OPE in the intermediate states yield recoupling coefficients. Figure adapted from Ref. [4].

$n=1$ and $r=3$,

$$
\begin{aligned}
\sigma_{3}^{( \pm)}\left(\sigma_{1}^{\prime \prime}\right) & =m_{1}^{2}+m_{2}^{2}-\frac{1}{2 \sigma_{1}^{\prime \prime}}\left(\sigma_{1}^{\prime \prime}-s+m_{1}^{2}\right)\left(\sigma_{1}^{\prime \prime}+m_{2}^{2}-m_{3}^{2}\right) \\
& \pm \frac{1}{2 \sigma_{1}^{\prime \prime}} \lambda^{1 / 2}\left(s, \sigma_{1}^{\prime \prime}, m_{1}^{2}\right) \lambda^{1 / 2}\left(\sigma_{1}^{\prime \prime}, m_{2}^{2}, m_{3}^{2}\right)
\end{aligned}
$$

Eq. 4.19 is illustrated in Fig. 4.7. Appendix C contains a sketch of the derivation of the amputated PWIS unitarity relations. The first term of Eq. 4.19 involves the direct propagation of an isobar in the intermediate state, whereas the second, third, and fourth term involve the exchange of a particle between isobars. The rescattering between isobars modifies the line shape of the isobar amplitudes 89,90 . The final term is the contribution from the OPE process, which gives and additional imaginary part to the amplitude in the physical region. At this stage we have not factored out the threshold factors from partial waves. This is straightforward to implement, however we do not do it here as we consider angular momenta in $S$-wave in the next chapter.

We have used $S$-matrix theory to construct the unitarity relations for the $\mathbf{3} \rightarrow \mathbf{3}$ scattering amplitude. The isobar model was used to aid in the construct and provide a systematic method to truncate higher partial waves. In the following chapter, we introduce an on-shell representation for 
the PWIS amplitude which satisfy the unitarity relations in Eq. 4.19). We call this the $B$-matrix representation, and we will investigate some of its analytic properties. 


\section{Chapter 5}

\section{THE B-MATRIX REPRESENTATION}

In the previous chapter, we established the unitarity constraints and isobar representation for elastic $\mathbf{3} \rightarrow \mathbf{3}$ scattering. We use the isobar representation to describe the $\mathbf{3} \rightarrow \mathbf{3}$ amplitude, $\mathcal{A}=\sum \mathcal{A}_{k j}$, where the indices $k$ and $j$ label the spectator particle in the final and initial state, respectively. We refer to the $\mathcal{A}_{k j}$ 's as isobar-spectator amplitudes, since they can be pictured as scattering of a quasi-particle, the isobar, and a stable spectator. The latter are expanded in partial waves of the three-particle system. Unitarity constrains the $\mathbf{3} \rightarrow \mathbf{3}$ amplitudes on the real energy axis, which results in specific relations involving the imaginary parts of the partial-wave-projected isobar-spectator amplitudes. Unitarity alone does not uniquely specify partial wave amplitudes, as evident, for example, in the $K$-matrix parametrization of $\mathbf{2} \rightarrow \mathbf{2}$ scattering amplitudes [51, 60]. We now discuss a specific parameterization for the isobar-spectator amplitudes which satisfies the three-body and two-body unitarity. It is given as a solution of a set of linear integral equations that involve, among others, the one particle exchange (OPE) as a driving term. We call this the $B$-matrix parameterization and it satisfies,

$$
\mathcal{A}_{k j}=\mathcal{B}_{k j}+\mathcal{B}_{k n} \tau_{n} \mathcal{A}_{n j}
$$

where $\mathcal{B}$ is the driving term that contains the OPE, $\tau$ is a known function of the phase space and of the $\mathbf{2} \rightarrow \mathbf{2}$ amplitudes. The product formally represents an integration over the intermediate isobar mass. In contrast to Ref. [84], we restrict the domain of the integrals to physical values of energies. This enables us to use the experimentally accessible subchannel amplitudes and we also 
discuss the consequences of this restriction. We derive Eq. 5.1 for isobars with arbitrary spin $s$, and for any value of the isobar-spectator orbital angular momentum $\ell$.

The $B$-matrix parameterization can be analytically continued to the complex energy plane and in Sec. 5.2 we discuss aspects of its analytic properties. Specifically, the one particle exchange process has some unique features, as it contains a kinematic singularity due to the exchange of a real particle, which can be isolated from the full $\mathbf{3} \rightarrow \mathbf{3}$ scattering amplitude. In addition, we also study the triangle amplitude that emerges from the $B$-matrix parameterization, and the relation to the Bethe-Salpeter solution.

\subsection{DESCRIPTION OF THE $B$-MATRIX REPRESENTATION}

Motivated by $S$-matrix theory, we present a parameterization for the PWIS amplitudes that satisfies real axis unitarity given by Eq. (4.19). In the $\mathbf{2} \rightarrow \mathbf{2}$ case, the $K$-matrix, $f^{-1}=K^{-1}-i \rho_{2}$, is an example of a parameterization satisfying unitarity. For the $\mathbf{3} \rightarrow \mathbf{3}$ case, we present the $B$-matrix parameterization for the PWIS amplitudes. The $B$-matrix parameterization is a linear integral equation for the amputated PWIS amplitudes that satisfy the unitarity relations Eq. (4.19):

$$
\begin{aligned}
\widetilde{\mathcal{A}}_{\ell_{k}^{\prime} s_{k}^{\prime} ; \ell_{j} s_{j}}^{J} & \left(\sigma_{k}^{\prime}, s, \sigma_{j}\right)=\widetilde{\mathcal{B}}_{\ell_{k}^{\prime} s_{k}^{\prime} ; \ell_{j} s_{j}}^{J}\left(\sigma_{k}^{\prime}, s, \sigma_{j}\right) \\
& +\sum_{n} \sum_{\ell_{n}^{\prime \prime}, s_{n}^{\prime \prime}} \int_{\sigma_{n}^{(\mathrm{th})}}^{\left(\sqrt{s}-m_{n}\right)^{2}} d \sigma_{n}^{\prime \prime} \widetilde{\mathcal{B}}_{\ell_{k}^{\prime} s_{k}^{\prime} ; \ell_{n}^{\prime \prime} s_{n}^{\prime \prime}}^{J}\left(\sigma_{k}^{\prime}, s, \sigma_{n}^{\prime \prime}\right) \tau_{n}\left(s, \sigma_{n}^{\prime \prime}\right) \widetilde{\mathcal{A}}_{\ell_{n}^{\prime \prime} s_{n}^{\prime \prime} ; \ell_{j} s_{j}}^{J}\left(\sigma_{n}^{\prime \prime}, s, \sigma_{j}\right),
\end{aligned}
$$

where the $B$-matrix $\widetilde{\mathcal{B}}_{\ell_{k}^{\prime} s_{k}^{\prime} ; \ell_{j} s_{j}}^{J}$ contains two terms,

$$
\widetilde{\mathcal{B}}_{\ell_{k}^{\prime} s_{k}^{\prime} ; \ell_{j} s_{j}}^{J}=\widetilde{\mathcal{R}}_{\ell_{k}^{\prime} s_{k}^{\prime} ; \ell_{j} s_{j}}^{J}+\widetilde{\mathcal{E}}_{\ell_{k}^{\prime} s_{k}^{\prime} ; \ell_{j} s_{j}}^{J}
$$

The function $\widetilde{\mathcal{E}}_{\ell_{k}^{\prime} s_{k}^{\prime} ; \ell_{j} s_{j}}^{J}$ is the amputated partial wave OPE amplitude, $\widetilde{\mathcal{R}}_{\ell_{k}^{\prime} s_{k}^{\prime} ; \ell_{j} s_{j}}^{J}$ is a real function that represents the short-distance three-body interactions unconstrained by unitarity, and $\tau_{n}$ is the product of the isobar-spectator phase space between and of the isobar amplitude

$$
\tau_{n}\left(s, \sigma_{n}\right)=\rho_{3}\left(s, \sigma_{n}\right) f_{s_{n}}\left(\sigma_{n}\right),
$$


with

$$
\rho_{3}\left(s, \sigma_{n}\right)=\frac{1}{64 \pi^{3}} \frac{2\left|\mathbf{p}_{n}^{\star}\right|}{\sqrt{s}} .
$$

The parameterization is diagrammatically represented in Fig. 5.1. The OPE amplitude is defined as

$$
\mathcal{E}_{k j}\left(\mathbf{p}^{\prime} ; \mathbf{p}\right)=\mathcal{F}_{k}\left(\mathbf{p}^{\prime} ; \mathbf{p}\right) \frac{1}{\mu_{j k}^{2}-u_{j k}-i \epsilon} \mathcal{F}_{j}\left(\mathbf{p}^{\prime} ; \mathbf{p}\right)
$$

where we note that the OPE only contributes to off-diagonal amplitudes, i.e. $j \neq k$. In principle, the OPE could contain a regular function of the energy in addition to the pole term, however unitarity only constrains the pole, and we assume all other real functions to be absorbed by $\mathcal{R}$. The amputated partial wave projected OPE amplitude $\widetilde{\mathcal{E}}_{\ell_{k}^{\prime} s_{k}^{\prime} ; \ell_{j} s_{j}}^{J}$ can be constructed using Eqs. 4.15 and (4.18). By construction, $\mathcal{R}$ is defined to have no threshold singularities in the three-particle physical region. The $\mathcal{R}$ represents the freedom of short-distance physics for the scattering of three particles, and can be any real function. The function $\mathcal{R}$ contains information on three body resonances, and can be modeled with pole terms similar to the $K$-matrix in the $\mathbf{2} \rightarrow \mathbf{2}$ case, so long as there are no singularities in the physical region of the amplitude. In an effective field theory approach, it represents a low order polynomial of contact interactions. For simplicity, in the following we assume the latter for $\mathcal{R}$. Appendix $\mathrm{D}$ illustrates how the $B$-matrix parameterizations satisfies the amputated PWIS unitarity relations. Aspects of its analytical properties are examined in Sec. 5.2 .

The $B$-matrix parameterization in Eq. (5.2) differs from Mai et al. [84] in the lower limit of the integral: the latter is derived using Lippman-Schwinger equations with a relativistic potential model, and includes contributions from the unphysical subthreshold region, $\sigma_{n}<\sigma_{n}^{(\text {th })}$. Obviously, both parameterization have the same imaginary part in the physical region, since both satisfy unitarity.

For notational simplicity, let $\widetilde{\mathcal{A}}_{k j}(s) \equiv \widetilde{\mathcal{A}}_{\ell_{k}^{\prime}, s_{k}^{\prime} ; \ell_{j}, s_{j}}^{J}\left(\sigma_{k}^{\prime}, s, \sigma_{j}\right)$, so that the amplitudes are matrices in the isobar sub-energies and angular momenta, which are indicated by the spectator indices. 

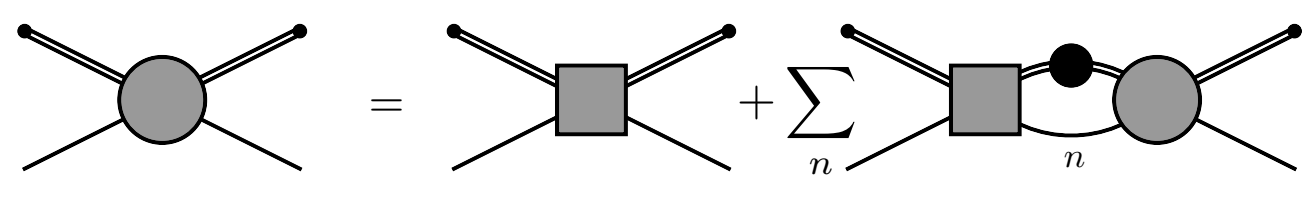

(a)

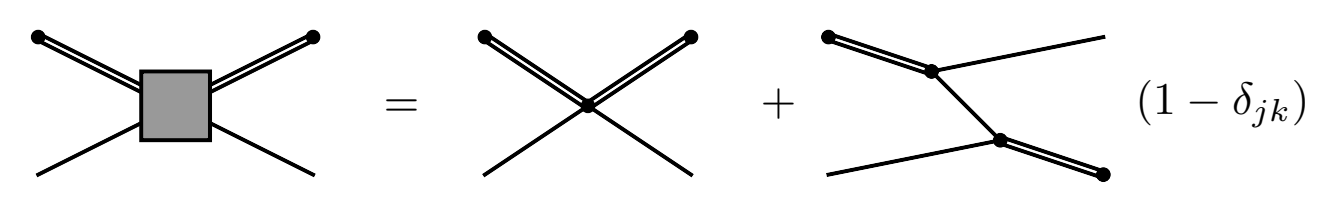

(b)

Figure 5.1: (a) Diagrammatic representation of the $B$-matrix parameterization in Eq. (5.2). The gray disk represent the amputated PWIS, and the gray box the $B$-matrix. (b) The $B$-matrix is composed of a short-range real $\mathcal{R}$ amplitude, and the OPE $\mathcal{E}$, see Eq. (5.3). Figure adapted from Ref. 4.

Equation $(5.2)$ is then a matrix relation with the integrations over intermediate isobars formally represented as matrix multiplications. Recalling that we work with the convention that isobars exists only in the (12) and (32) channels, we write the $B$-matrix parameterization as the set of coupled equations

$$
\begin{aligned}
& \widetilde{\mathcal{A}}_{13}(s)=\widetilde{\mathcal{B}}_{13}(s)+\widetilde{\mathcal{B}}_{13}(s) \tau_{3}(s) \widetilde{\mathcal{A}}_{33}(s) \\
& \widetilde{\mathcal{A}}_{33}(s)=\widetilde{\mathcal{B}}_{31}(s) \tau_{1}(s) \widetilde{\mathcal{A}}_{13}(s)
\end{aligned}
$$

with the other two amplitudes given by a similar set of equations,

$$
\begin{aligned}
& \widetilde{\mathcal{A}}_{31}(s)=\widetilde{\mathcal{B}}_{31}(s)+\widetilde{\mathcal{B}}_{31}(s) \tau_{1}(s) \widetilde{\mathcal{A}}_{11}(s) \\
& \widetilde{\mathcal{A}}_{11}(s)=\widetilde{\mathcal{B}}_{13}(s) \tau_{3}(s) \widetilde{\mathcal{A}}_{31}(s)
\end{aligned}
$$

The Eqs. 5.7) can be combined into one integral equation for $\widetilde{\mathcal{A}}_{13}$,

$$
\widetilde{\mathcal{A}}_{13}(s)=\widetilde{\mathcal{B}}_{13}(s)+\mathcal{K}_{11}(s) \tau_{1}(s) \widetilde{\mathcal{A}}_{13}(s)
$$

where the kernel $\mathcal{K}_{11}$ is

$$
\mathcal{K}_{11}(s)=\widetilde{\mathcal{B}}_{13}(s) \tau_{3}(s) \widetilde{\mathcal{B}}_{31}(s)
$$


Similarly, Eqs. (5.8) give

$$
\widetilde{\mathcal{A}}_{31}(s)=\widetilde{\mathcal{B}}_{31}(s)+\mathcal{K}_{33}(s) \tau_{3}(s) \widetilde{\mathcal{A}}_{31}(s)
$$

where the kernel $\mathcal{K}_{33}$ is given by exchanging the $1 \leftrightarrow 3$ indices in Eq. (5.10). Eqs. (5.9) and (5.11) can be formally inverted to yield the solutions,

$$
\begin{aligned}
& \widetilde{\mathcal{A}}_{13}(s)=\left[\mathbb{1}-\mathcal{K}_{11}(s) \tau_{1}(s)\right]^{-1} \widetilde{\mathcal{B}}_{13}(s), \\
& \widetilde{\mathcal{A}}_{33}(s)=\left[\mathbb{1}-\mathcal{K}_{33}(s) \tau_{3}(s)\right]^{-1} \mathcal{K}_{33}(s), \\
& \widetilde{\mathcal{A}}_{31}(s)=\left[\mathbb{1}-\mathcal{K}_{33}(s) \tau_{3}(s)\right]^{-1} \widetilde{\mathcal{B}}_{31}(s), \\
& \widetilde{\mathcal{A}}_{11}(s)=\left[\mathbb{1}-\mathcal{K}_{11}(s) \tau_{1}(s)\right]^{-1} \mathcal{K}_{11}(s),
\end{aligned}
$$

Several terms can be identified in the kernels, $\mathcal{K}_{k j}(s)=\mathcal{G}_{k j}(s)+\mathcal{H}_{k j}(s)+\mathcal{T}_{k j}^{(1)}(s)+\mathcal{T}_{k j}^{(2)}(s)$, where $\mathcal{G}$ is a bubble diagram, $\mathcal{H}$ is a box diagram, and the $\mathcal{T}$ 's are triangle diagrams, generated by integrals over OPE and contact terms in Eq. (5.3). Explicitly,

$$
\begin{aligned}
\mathcal{G}_{k j}(s) & =\sum_{n} \widetilde{\mathcal{R}}_{k n}(s) \tau_{n}(s) \widetilde{\mathcal{R}}_{n j}(s), \\
\mathcal{T}_{k j}^{(1)}(s) & =\sum_{n} \widetilde{\mathcal{E}}_{k n}(s) \tau_{n}(s) \widetilde{\mathcal{R}}_{n j}(s), \\
\mathcal{T}_{k j}^{(2)}(s) & =\sum_{n} \widetilde{\mathcal{R}}_{k n}(s) \tau_{n}(s) \widetilde{\mathcal{E}}_{n j}(s), \\
\mathcal{H}_{k j}(s) & =\sum_{n} \widetilde{\mathcal{E}}_{k n}(s) \tau_{n}(s) \widetilde{\mathcal{E}}_{n j}(s)
\end{aligned}
$$

These diagrams occur in the denominators of the amplitudes in Eqs. (5.12), cf. Fig. 5.2, They differ to the Feynman diagrams obtained in a perturbative QFT since the integrations are only over the physical region, changing the analytic structure below threshold (see Sec. 5.2).

The solutions can be interpreted as an infinite series of exchange and bubble diagrams. For example, expanding the solution for $\widetilde{\mathcal{A}}_{13}$,

$$
\begin{aligned}
\widetilde{\mathcal{A}}_{13}(s) & =\widetilde{\mathcal{B}}_{13}(s)+\mathcal{K}_{11}(s) \tau_{1}(s) \widetilde{\mathcal{B}}_{13}(s) \\
& +\mathcal{K}_{11}(s) \tau_{1}(s) \mathcal{K}_{11}(s) \tau_{1}(s) \widetilde{\mathcal{B}}_{13}(s)+\cdots
\end{aligned}
$$




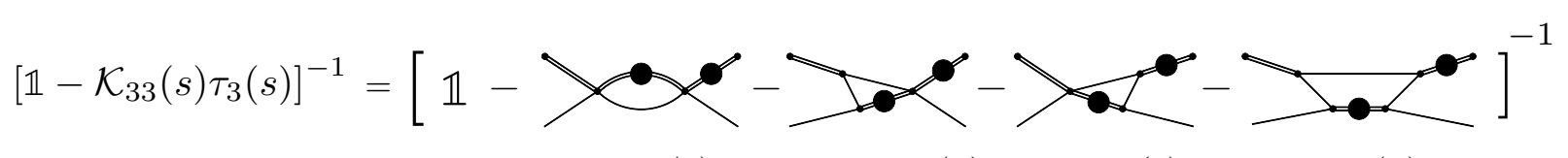

(a)

(b)

(c)

(d)

Figure 5.2: The denominator of the $B$-matrix parameterization contains four primitive diagrams associated with the rescattering of the $B$-matrix: (a) bubble diagram, (b) and (c) triangle diagrams, and (d) box diagram. Figure adapted from Ref. 4].

The first term is the OPE and contact interaction, the second term is a ladder diagram with three exchanges, and various combinations of bubbles and OPE, and so on. The unitarization of bubble diagrams has been considered in quasi-two-body models $[1,91,93]$. In these models it is easy to show how additional cuts appear in the unphysical sheets due to the isobar decay.

Three-body resonances manifest as poles in the complex $s$-plane of the scattering amplitude. Rearranging the constituents of the kernel relates the two denominators

$$
\begin{aligned}
\widetilde{\mathcal{B}}_{13}(s) \tau_{3}(s) & {\left[\mathbb{1}-\mathcal{K}_{33}(s) \tau_{3}(s)\right]^{-1} } \\
& =\left[\mathbb{1}-\mathcal{K}_{11}(s) \tau_{1}(s)\right]^{-1} \widetilde{\mathcal{B}}_{13}(s) \tau_{3}(s) .
\end{aligned}
$$

Thus, we can write the full $\mathbf{3} \rightarrow \mathbf{3}$ amplitude in terms of a single Fredholm determinant. The determinants are independent of the external isobar energies, and the intermediate integrations will modify the phase space factors to incorporate rescattering effects. Resonance poles can be determined by solving

$$
\operatorname{det}\left[\mathbb{1}-\mathcal{K}_{11}(s) \tau_{1}(s)\right]=0
$$

The $B$-matrix solutions are real-boundary values of analytic functions in the complex $s$-plane. The physical amplitudes are defined by $s \rightarrow s+i \epsilon, \sigma_{j} \rightarrow \sigma_{j}+i \epsilon$, and $\sigma_{k}^{\prime} \rightarrow \sigma_{k}^{\prime}+i \epsilon$. Aspects of its analytic properties are discussed in the following section. 


\subsection{ASPECTS OF ANALYTIC PROPERTIES}

In this section, we examine the singularities of the OPE amplitude and the triangle diagram from the $B$-matrix parameterization. We numerically evaluate an amplitude where all external particles have unit mass $\left(m_{1}=m_{2}=m_{3}=1\right)$ and coupling. In these studies, the units are arbitrary. For simplicity, we consider $S$-waves only, i.e. $J\left(\ell^{\prime} s^{\prime}\right)_{k}(\ell s)_{j}=0(00)_{k}(00)_{j}$. Generalizing to nonzero angular momenta does not change the analytic properties.

\subsubsection{ONE PARTICLE EXCHANGE}

As seen in Eqs. (5.13), the building block for the $B$-matrix kernels is the OPE amplitude. Projecting Eq. (5.6) using Eq. 4.15 gives the $S$-wave OPE amplitude,

$$
\widetilde{\mathcal{E}}_{k j}^{S}\left(\sigma_{k}^{\prime}, s, \sigma_{j}\right)=\frac{1}{4\left|\mathbf{p}_{k}^{\prime \star}\right|\left|\mathbf{p}_{j}^{\star}\right|} \log \left(\frac{z_{k j}-1}{z_{k j}+1}\right)
$$

where $z_{k j}$ is given as

$$
z_{k j}=\frac{2 s\left(\sigma_{j}+m_{k}^{2}-\mu_{j k}^{2}\right)-\left(s+\sigma_{j}-m_{j}^{2}\right)\left(s+m_{k}^{2}-\sigma_{k}^{\prime}\right)}{\lambda^{1 / 2}\left(s, \sigma_{j}, m_{j}^{2}\right) \lambda^{1 / 2}\left(s, \sigma_{k}^{\prime}, m_{k}^{2}\right)},
$$

where $\lambda(a, b, c)=a^{2}+b^{2}+c^{2}-2(a b+b c+c a)$ is the Källén triangle function. Eq. (5.18). We investigate the OPE as a function of $s$ for fixed real $\sigma_{j}$ and $\sigma_{k}^{\prime}$. The imaginary part of the OPE is

$$
\operatorname{Im} \widetilde{\mathcal{E}}_{k j}^{S}\left(\sigma_{k}^{\prime}, s, \sigma_{j}\right)=\frac{\pi}{4\left|\mathbf{p}_{j}^{\star}\right|\left|\mathbf{p}_{k}^{\prime \star}\right|} \Theta\left(1-\left|z_{k j}\right|^{2}\right),
$$

which is given by the unitarity relations in Eq. 4.19). The OPE has four branch points in $s$, one at zero, one at infinity, and two which we label $s_{k j}^{( \pm)}$,

$$
\begin{aligned}
s_{k j}^{( \pm)} & =\frac{1}{2 \mu_{j k}^{2}}\left[\left(m_{k}^{2}-\sigma_{j}\right)\left(m_{j}^{2}-\sigma_{k}^{\prime}\right)-\mu_{j k}^{4}\right. \\
& +\mu_{j k}^{2}\left(m_{k}^{2}+m_{j}^{2}+\sigma_{j}+\sigma_{k}^{\prime}\right) \\
& \left. \pm \lambda^{1 / 2}\left(\mu_{j k}^{2}, m_{k}^{2}, \sigma_{j}\right) \lambda^{1 / 2}\left(\mu_{j k}^{2}, m_{j}^{2}, \sigma_{k}^{\prime}\right)\right],
\end{aligned}
$$

which depend on the isobar invariant masses. The momenta in the denominator do not contribute additional branch points, because the logarithm vanishes and cancel the singularity, as expected 
from the $S$-wave threshold behavior. The $s_{k j}^{( \pm)}$branch points are in general complex. There are then two branch cuts: one where $s \in(-\infty, 0]$, called the virtual particle exchange (VPE) cut, and one connecting $s_{k j}^{(-)}$to $s_{k j}^{(+)}$, called the real particle exchange (RPE) cut. The VPE cut is associated with the exchange of a virtual particle, generating long-range forces. Historically, the RPE cut is associated with the exchange of a real particle between isobars, i.e. when it is kinematically allowed for an isobar to decay. This corresponds to when the RPE branch points lie on the real axis above the isobar-spectator threshold. If the isobar invariant masses are below the decay threshold, then the RPE branch points move in the complex plane below the isobar-spectator threshold. For convenience, however, we will always call this the RPE cut, and emphasize that a real particle exchange occurs only if it is kinematically accessible. Note that although the value of the isobar mass dictates the physics of the OPE, the OPE is blind to the decay products of the isobar and the physical threshold in $s$ is $\max \left\{\left(\sqrt{\sigma_{j}}+m_{j}\right)^{2},\left(\sqrt{\sigma_{k}^{\prime}}+m_{k}\right)^{2}\right\}$.

We can understand the analytic structure of the OPE by writing a dispersive representation in s. Eq. (5.19) is nonzero in two regions, leading to the relation

$$
\begin{aligned}
\widetilde{\mathcal{E}}_{k j}^{S}\left(\sigma_{k}^{\prime}, s, \sigma_{j}\right) & =\int_{\Gamma_{V}} d s^{\prime} \frac{1}{s^{\prime}-s-i \epsilon} \frac{1}{4\left|\mathbf{p}_{k}^{\prime \star}\right|\left|\mathbf{p}_{j}^{\star}\right|} \\
& +\int_{\Gamma_{R}} d s^{\prime} \frac{1}{s^{\prime}-s-i \epsilon} \frac{1}{4\left|\mathbf{p}_{k}^{\prime \star}\right|\left|\mathbf{p}_{j}^{\star}\right|},
\end{aligned}
$$

where $\Gamma_{V}$ is the contour over the VPE cut and $\Gamma_{R}$ is the contour over the RPE cut. The integrand has four branch points associated with the thresholds and pseudo-thresholds of the initial and final momenta. We choose to orient the branch cuts such that the lowest branch point $\left(\min \left\{\left(\sqrt{\sigma_{k}^{\prime}}-\right.\right.\right.$ $\left.\left.\left.m_{k}\right)^{2},\left(\sqrt{\sigma_{j}}-m_{j}\right)^{2}\right\}\right)$ has a cut running to $-\infty$, the highest branch point $\left(\max \left\{\left(\sqrt{\sigma_{k}^{\prime}}+m_{k}\right)^{2},\left(\sqrt{\sigma_{j}}+\right.\right.\right.$ $\left.\left.m_{j}\right)^{2}\right\}$ ) has a cut running to $+\infty$, and the other two branch points have a branch cut joining them. The contour $\Gamma_{V}$ is always taken above the real axis, whereas the contour $\Gamma_{R}$ depends on the external masses. The physical amplitude is defined as the boundary value when $s \rightarrow s+i \epsilon$, below the RPE cut.

For fixed $\sigma_{j}>\sigma_{j}^{(\mathrm{th})}$, the RPE cut can be categorized by different regions in $\sigma_{k}^{\prime}$. In Fig. 5.3 . we illustrate how the analytic structure of the integrand and the integration contours change in 


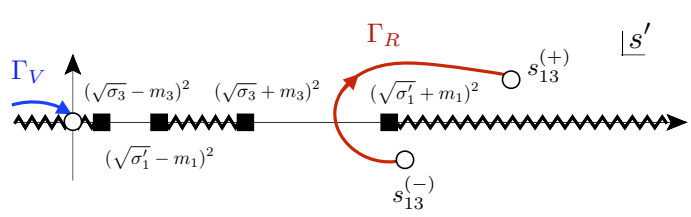

(a)

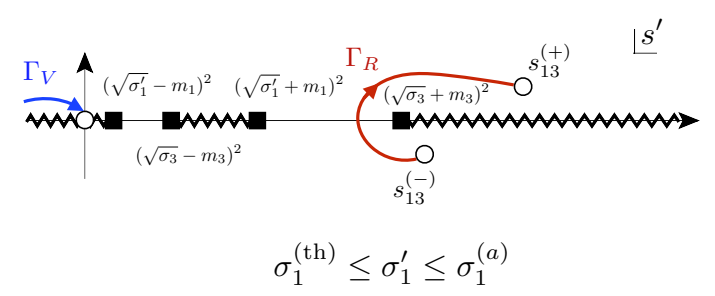

(c)

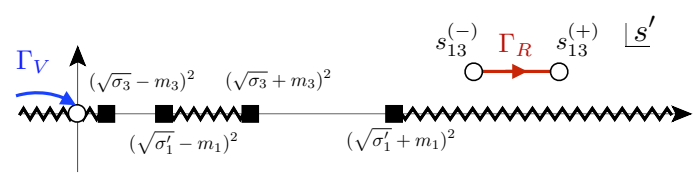

(b)

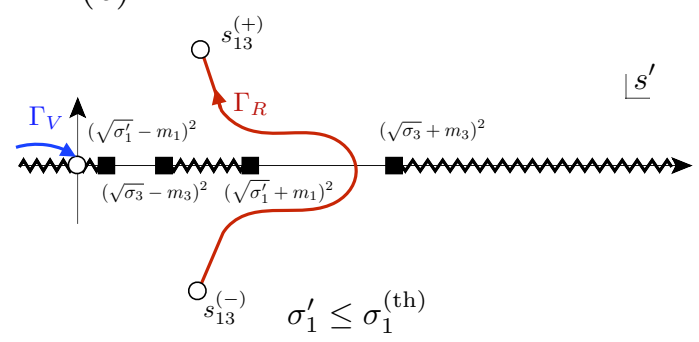

(d)

Figure 5.3: Cut structure of the OPE integrand Eq. (5.19) in the $s^{\prime}$-plane, and the OPE integration paths for the RPE contour ( $\Gamma_{R}$ in red) and the VPE contour ( $\Gamma_{V}$ in blue) for the dispersive integral Eq. (5.21). The four cases as a function of $\sigma_{1}^{\prime}$ are: (a) $\sigma_{1}^{\prime} \geq \sigma_{1}^{(b)}$, (b) $\sigma_{1}^{(a)} \leq \sigma_{1}^{\prime} \leq \sigma_{1}^{(b)}$, (c) $\sigma_{1}^{(\text {th })} \leq \sigma_{1}^{\prime} \leq \sigma_{1}^{(a)}$, and (d) $\sigma_{1}^{\prime} \leq \sigma_{1}^{(\text {th })}$. Real particle exchange cannot occur in case $(\mathrm{d})$. In the logarithmic representation Eq. (5.17), the RPE cut is circular. Figure adapted from Ref. [4].

these regions. Assuming a small imaginary part $\mu_{j k}^{2} \rightarrow \mu_{j k}^{2}-i \epsilon$, the RPE branch points have a finite imaginary part for $\sigma_{k}^{\prime}<\sigma_{k}^{(\text {th })}$, with opposite signs. When $\sigma_{k}^{\prime}>\sigma_{k}^{(\text {th })}$, the branch points are infinitesimally close to the real axis. In the physical region, $s_{k j}^{(-)}$has inflection points at two locations of $\sigma_{k}^{\prime}$ :

$$
\begin{aligned}
\sigma_{k}^{(a)} & =m_{j}^{2}+\mu_{j k}^{2}+\frac{m_{j}\left(\sigma_{j}+\mu_{j k}^{2}-m_{k}^{2}\right)}{\sqrt{\sigma_{j}}}, \\
\sigma_{k}^{(b)} & =-\frac{1}{2 m_{k}^{2}}\left[2 m_{k}^{2}\left(\sigma_{j}-m_{j}^{2}\right)-m_{k}^{4}\right. \\
& -\left(\sigma_{j}-\mu_{j k}^{2}\right)^{2}+\left(m_{k}^{2}+\mu_{j k}^{2}-\sigma_{j}\right) \\
& \left.\times \sqrt{4 m_{j}^{2} m_{k}^{2}+\lambda\left(\sigma_{j}, \mu_{j k}^{2}, m_{k}^{2}\right)}\right]
\end{aligned}
$$

which follow from $d s_{k j}^{(-)} / d \sigma_{k}^{\prime}=0$ corresponding to $\operatorname{Im} s_{k j}^{(-)}=0$. Both $\sigma_{k}^{(a)}$ and $\sigma_{k}^{(b)}$ correspond to when $s_{k j}^{(-)}$crosses the real $s$-axis. The $s_{k j}^{(+)}$branch point always lies in the upper-half plane. 


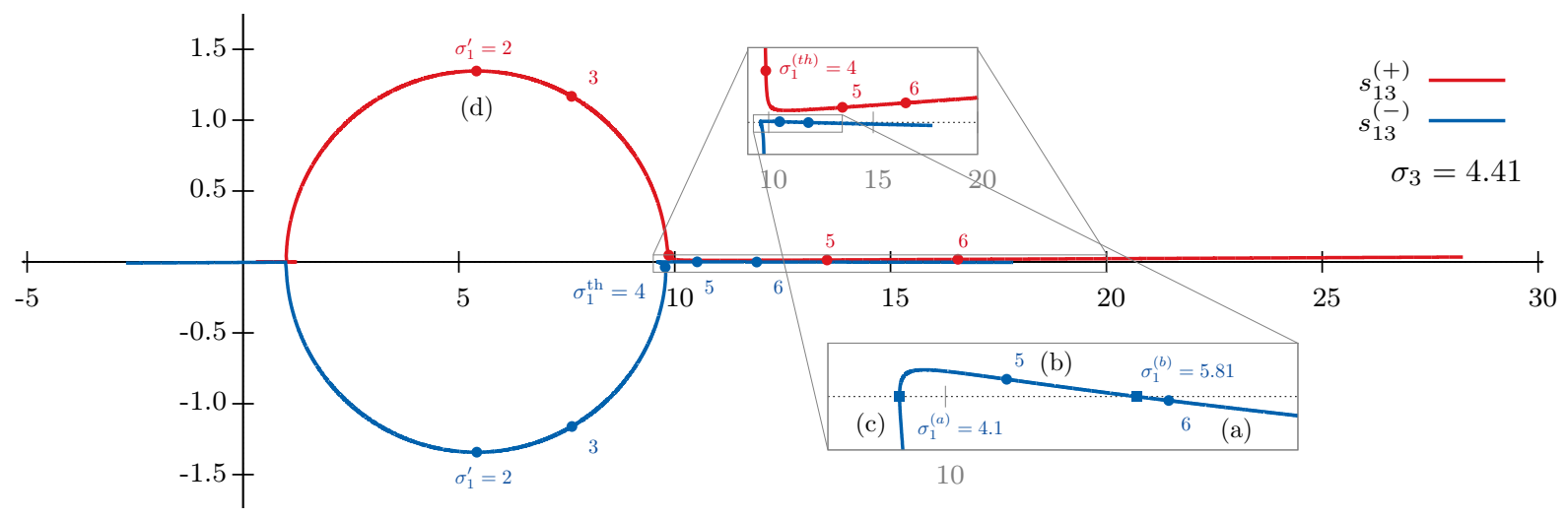

Figure 5.4: Motion of $s_{13}^{( \pm)}$in the $s$-plane as a function of $\sigma_{1}^{\prime}$ for fixed $\sigma_{3}=4.41$, and unit external masses, $m_{1}=m_{2}=m_{3}=1$. Shown in red is $s_{13}^{(+)}$and in blue $s_{13}^{(-)}$. The points indicate various $\sigma_{1}^{\prime}$ values. Note that the physical region begins at $\sigma_{1}^{\prime}=4$. The inset shows the infinitesimal region where $s_{13}^{(-)}$curve crosses the real axis at the points $\sigma_{1}^{(a)}=4.1$ and $\sigma_{1}^{(b)}=5.81$. The labels (a)-(d) indicate the regions described in the text and depicted in Fig. 5.3. Figure adapted from Ref. [4].

We can therefore classify the regions according to when the RPE branch points are both in the upper-half plane or when they approach the real axis.

(a) $\sigma_{k}^{\prime} \geq \sigma_{k}^{(b)}$, see region (a) in Fig. 5.4. Here $s_{k j}^{(-)}$is below the real axis, and $s_{k j}^{(+)}$is above the real axis. The RPE cut connects these two points by crossing the real axis below the threshold $\left(\sqrt{\sigma_{k}^{\prime}}+m_{k}\right)^{2}$. Real particle exchange in this case has consequences when considering the OPE processes embedded in the triangle diagram, which is discussed in the next section. For $k=1$ and $j=3$, Fig. 5.3(a) shows the RPE and VPE contours, $\Gamma_{R}$ and $\Gamma_{V}$, respectively. Note that in the logarithmic representation, the RPE cut is circular, whereas in the dispersive representation, one can define the cut in any chosen manner as long as singularities are not crossed.

(b) $\sigma_{k}^{(a)} \leq \sigma_{k}^{\prime} \leq \sigma_{k}^{(b)}$, see region (b) in Fig. 5.4. When $\sigma_{k}^{\prime}$ decreases below the inversion point $\sigma_{k}^{(b)}, s_{k j}^{(-)}$wanders above the real axis. The RPE cut directly connects $s_{k j}^{(-)}$to $s_{k j}^{(+)}$without crossing the real axis. This is the typical case when considering the exchange of a real particle, 
illustrated in Fig. 5.3(b). Note that when $\sigma_{k}^{\prime}=\sigma_{j}$ for equal masses $m_{j}=m_{k}$, then the integrand branch points merge into pole singularities.

(c) $\sigma_{k}^{(\mathrm{th})} \leq \sigma_{k}^{\prime} \leq \sigma_{k}^{(a)}$, see region (c) in Fig. 5.4. The RPE branch points again wrap around the real axis, cf. Fig. 5.3(c), with the cut crossing the real axis below the threshold $\left(\sqrt{\sigma_{j}}+m_{j}\right)^{2}$.

(d) $\sigma_{k}^{\prime} \leq \sigma_{k}^{(\text {th })}$, see region (d) in Fig. 5.4. The branch points $s_{k j}^{( \pm)}$move deep into the complex plane, as shown in Fig. 5.3(d). In this region, the isobar cannot decay, and therefore it is unphysical for the $\mathbf{3} \rightarrow \mathbf{3}$ elastic scattering. Real particle exchange cannot occur, leaving only the virtual contributions.

If we evaluate the OPE along the real $s$-axis in regions (a) or (c), we find that the real part of the OPE has a jump due to crossing the RPE cut. In the logarithmic representation, this crossing occurs when $z_{k j}=0$, that is, when

$$
\begin{aligned}
s_{k j}^{(0)} & =\frac{1}{2}\left[m_{j}^{2}+m_{k}^{2}-2 \mu_{j k}^{2}+\sigma_{j}+\sigma_{k}^{\prime}\right. \\
& +\left(4\left(m_{j}^{2}-\sigma_{j}\right)\left(m_{k}^{2}-\sigma_{k}^{\prime}\right)\right. \\
& \left.\left.+\left(m_{j}^{2}+m_{k}^{2}-2 \mu_{j k}^{2}+\sigma_{j}+\sigma_{k}^{\prime}\right)^{2}\right)^{1 / 2}\right] .
\end{aligned}
$$

When choosing a different contour for $\Gamma_{R}$ in the dispersive representation, the location of this crossing depends on where real axis crosses the chosen contour.

These cases are illustrated in Fig. 5.3 for spectators $k=1$ and $j=3$. We plot the OPE amplitudes, Eq. (5.17), as a function of $s$ for fixed $\sigma_{3}$ and $\sigma_{1}^{\prime}$ in Fig. 5.5. Figure 5.5(a) shows the OPE computed at $\sigma_{1}^{\prime}$ in region (a). At this energy, the $s_{k j}^{(-)}$is below the real axis, and the RPE cut wraps around the real axis, passing below the threshold $\left(\sqrt{\sigma_{1}^{\prime}}+m_{1}\right)^{2}$. The jump in the real part at $s_{13}^{(0)}$ is due to crossing the RPE cut. Figure 5.5(b), is evaluated at $\sigma_{1}^{\prime}$ in region (b), where both branch points are above the real axis. Here, we illustrate that as $\sigma_{1}^{\prime}$ decreases, the width of the imaginary part decreases and the peak increases. The narrowing imaginary region physically represents that less phase space is available for real particle propagation in the intermediate state. Figure $5.5(\mathrm{c})$ is computed for $\sigma_{1}^{\prime}$ in region (c), right above the two-particle threshold. There is a 

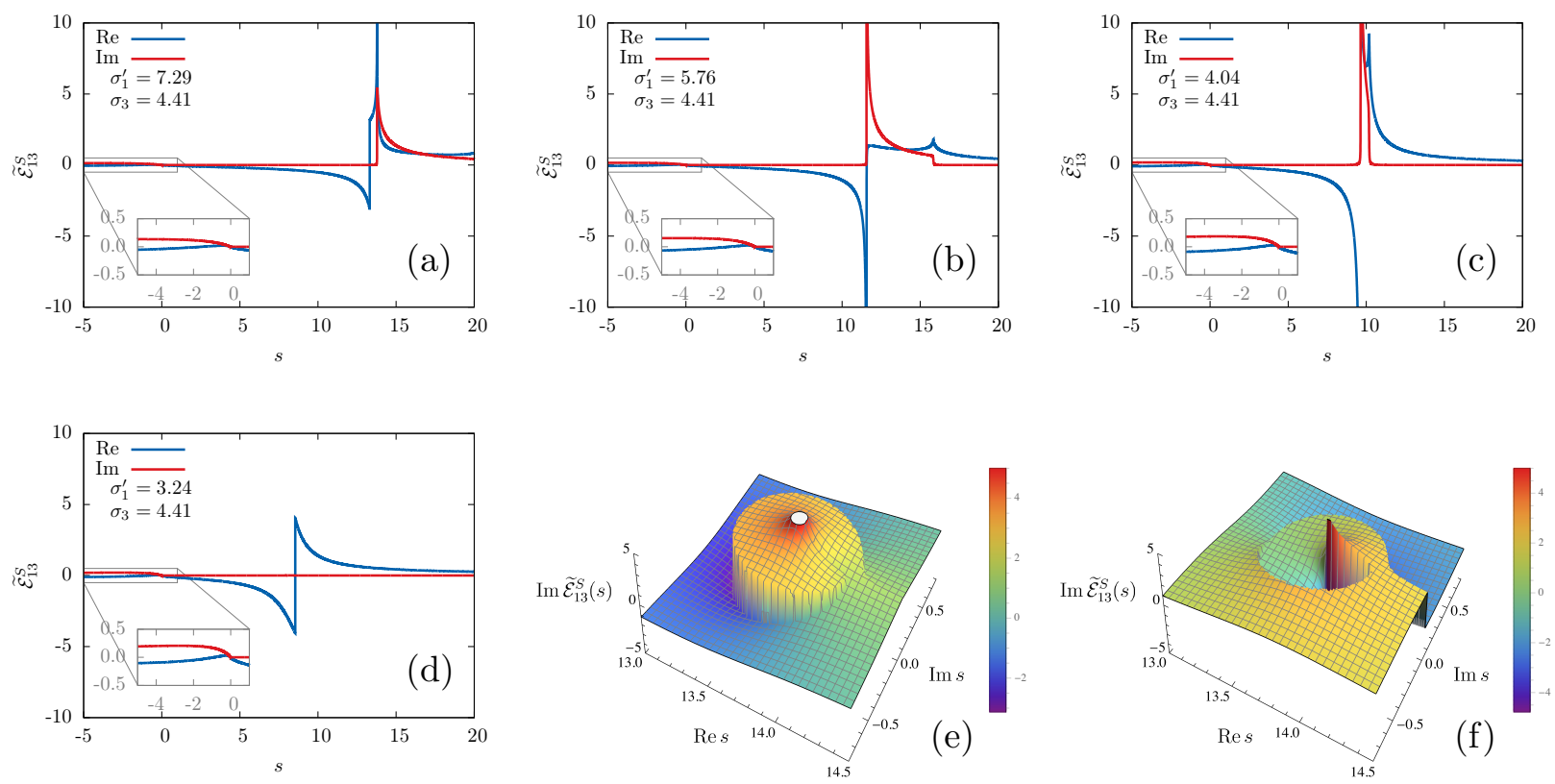

Figure 5.5: OPE amplitudes Eq. (5.17) for external masses $m_{1}=m_{2}=m_{3}=1$ at fixed $\sigma_{3}=4.41$ for the four regions depicted in Fig. 5.3. (a) $\sigma_{1}^{\prime}=3.24$ representing the unphysical region, (b) 4.04, where the RPE cut wraps around the real axis, (c) 4.41, where the RPE branch points are above the real axis, and (d) 7.29 where the RPE cut wraps around the real axis. The insets show the contribution from the VPE cut. For these values, the inflection points are $\sigma_{1}^{(a)}=4.1$ and $\sigma_{1}^{(b)}=5.81$. The real and imaginary parts of the OPE Eq. (5.17) at $\sigma_{1}^{\prime}=7.29$ in the complex $s$-plane are shown in (e) and (f), respectively. The discontinuity at $s=s_{13}^{(0)}=13.31$ occurs when evaluating the OPE across the RPE cut. Figure adapted from Ref. 4]. 
jump in the real part at $s_{13}^{(0)}$ from crossing the RPE cut. The final case is illustrated in Fig. $5.5(\mathrm{~d})$. where the OPE computed in the unphysical region $(\mathrm{d})$. There is an imaginary part due to the VPE cut only, as it is kinematically inaccessible for the exchange of a real particle. The jump in the real part at $s_{13}^{(0)}$ comes from crossing the RPE cut. Figures $5.5(\mathrm{e})$ and $5.5(\mathrm{f})$ shows a 3-dimensional plot of the real and imaginary part of the logarithmic representation of the OPE, Eq. (5.17). The circular cut is clearly visible connecting the RPE branch points. The physical region is taken as the region approaching the real axis, below the RPE cut.

To summarize, the analytic structure of the OPE is given by two branch cuts, the VPE and RPE cuts. The VPE cut is present for $-\infty<s \leq 0$, and is associated with the exchange of an off-shell particle. For physical isobars, the RPE cut is in the physical region. We have shown different scenarios, identified by the isobar masses, in which the RPE branch points can approach the physical region, which impact the structure of the $B$-matrix kernels.

\subsubsection{TRIANGLE DIAGRAMS}

To understand resonance poles of $\mathbf{3} \rightarrow \mathbf{3}$ systems, the analytic structure of the $B$-matrix parameterization, Eq. 5.12 must be understood in the complex $s$-plane. This means understanding the properties of the $B$-matrix kernels. Here, we investigate the triangle diagram, and leave the box diagram for future studies. Let us work with the triangle $\mathcal{T}_{B} \equiv \mathcal{T}_{11}^{(2)}$ introduced in Eq. (5.13c), where all angular momenta are in $S$-wave. For convenience, let $\widetilde{\mathcal{R}}=1$, thus the amplitudes are independent of $\sigma_{1}^{\prime}$, and given by

$$
\mathcal{T}_{B}(s)=\int_{\sigma_{3}^{(\mathrm{th})}}^{\left(\sqrt{s}-m_{3}\right)^{2}} d \sigma_{3}^{\prime \prime} \tau_{3}\left(s, \sigma_{3}^{\prime \prime}\right) \widetilde{\mathcal{E}}_{31}^{S}\left(\sigma_{3}^{\prime \prime}, s, \sigma_{1}\right),
$$

where $\tau_{3}\left(s, \sigma_{3}^{\prime \prime}\right)=\rho_{3}\left(s, \sigma_{3}^{\prime \prime}\right) D_{3}^{-1}\left(\sigma_{3}^{\prime \prime}\right)$, and the dependence of $\mathcal{T}_{B}$ on $\sigma_{1}$ has been understood. To ensure the correct analytic properties of the isobar amplitude, we introduce its dispersive representation

$$
f_{3}\left(\sigma_{3}^{\prime \prime}\right)=\frac{1}{\pi} \int_{\sigma_{3}^{(\text {th })}}^{\infty} d \widehat{\sigma} \frac{\operatorname{Im} f_{3}(\widehat{\sigma})}{\widehat{\sigma}-\sigma_{3}^{\prime \prime}-i \epsilon}
$$


giving the form for $\mathcal{T}_{B}$

$$
\mathcal{T}_{B}(s)=\frac{1}{\pi} \int_{\sigma_{3}^{\text {(th })}}^{\infty} d \widehat{\sigma} \operatorname{Im} f_{3}(\widehat{\sigma}) \int_{\sigma_{3}^{(\text {th })}}^{\left(\sqrt{s}-m_{3}\right)^{2}} d \sigma_{3}^{\prime \prime} \frac{\rho_{3}\left(s, \sigma_{3}^{\prime \prime}\right) \widetilde{\mathcal{E}}_{31}^{S}\left(\sigma_{3}^{\prime \prime}, s, \sigma_{1}\right)}{\widehat{\sigma}-\sigma_{3}^{\prime \prime}-i \epsilon}
$$

We see the $\sigma_{3}^{\prime \prime}$-integral does not depend on $f_{3}(\widehat{\sigma})$, so for simplicity we take the narrow width limit $\operatorname{Im} f_{3}(\widehat{\sigma})=\pi \delta\left(\widehat{\sigma}-M^{2}\right)$, where $M$ is the mass of the isobar. The narrow width limit shifts the unitarity cut in the triangle diagram to begin at the threshold $s=\left(M+m_{3}\right)^{2}$. However, for a general isobar shape, Eq. (5.26) can be used to sum over its distribution, recovering the correct unitarity branch cut starting at $s_{\mathrm{th}}$. Therefore, the triangle diagram has the form

$$
\mathcal{T}_{B}(s)=\int_{\sigma_{3}^{(\text {th })}}^{\left(\sqrt{s}-m_{3}\right)^{2}} d \sigma_{3}^{\prime \prime} \frac{\rho_{3}\left(s, \sigma_{3}^{\prime \prime}\right) \widetilde{\mathcal{E}}_{31}^{S}\left(\sigma_{3}^{\prime \prime}, s, \sigma_{1}\right)}{M^{2}-\sigma_{3}^{\prime \prime}-i \epsilon} .
$$

Figure 5.6 shows the triangle diagram in consideration. The $B$-matrix triangle contains singularities on the physical sheet. These are due to the $s$-singularities in $\rho_{3}$ and $\widetilde{\mathcal{E}}_{13}^{S}$, and to endpoint singularities when the integration limits hit the $\sigma_{3}^{\prime \prime}$-singularities of the integrand. The upper integration limit gives a branch cut for $s<0$. Since $\rho_{3}\left(s, \sigma_{3}^{\prime \prime}\right) \propto\left\{\left[\sigma_{3}^{\prime \prime}-\left(\sqrt{s}+m_{3}\right)^{2}\right]\left[\sigma_{3}^{\prime \prime}-\left(\sqrt{s}-m_{3}\right)^{2}\right]\right\}^{1 / 2} / s$, there is a pole at $s=0$. When the integration variable hits the lower limit $\sigma_{3}^{\prime \prime}=\sigma_{3}^{(\mathrm{th})}$, there are two branch point singularities at $s=\left(\sqrt{\sigma_{3}^{(\mathrm{th})}} \pm m_{3}\right)^{2}=\left(m_{1}+m_{2} \pm m_{3}\right)^{2}$. In the narrow width limit, the unitarity cut opens when the upper limit of the integral hits the pole in the isobar propagator, for $s=\left(M+m_{3}\right)^{2}$. On the real $s$-axis, the OPE has a discontinuity in the real part when $z_{31}=0$, i.e. at $\sigma_{3}^{\prime \prime}=\sigma_{3}^{(\mathrm{th})}$. This discontinuity from crossing the RPE cut is present in $\mathcal{T}_{B}$. Two more singularities occur when $\sigma_{3}^{\prime \prime}$ hits the two inflection points $\sigma_{3}^{(a)}$ and $\sigma_{3}^{(b)}$, which are defined in Eqs. (5.22). The OPE pinches the real axis at $\sigma_{3}^{\prime \prime}=\sigma_{3}^{(a)}$, and generates a singularity in $\mathcal{T}_{B}$ at the initial state threshold $s=\left(\sqrt{\sigma_{1}}+m_{1}\right)^{2}$. This can be understood by realizing that the OPE branch points, Eq. (5.20), can alternatively be written in terms of $\sigma_{3}^{\prime \prime}$ as a function of $s$ for fixed $\sigma_{1}$. The 


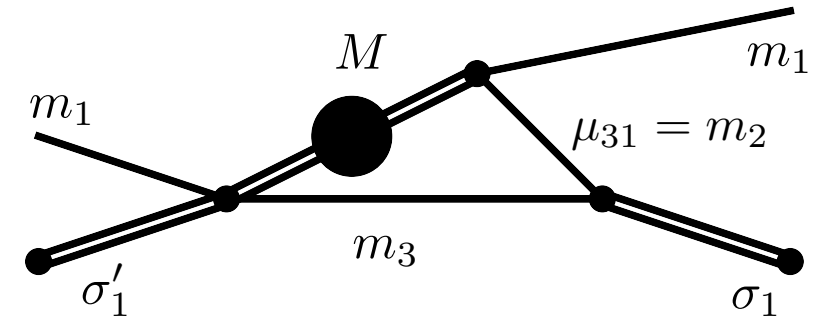

Figure 5.6: The triangle diagram $\mathcal{T}_{B}$ contribution to the kernel $\mathcal{K}_{11}$. We take the isobar to have a narrow width with mass $M$. For numerical evaluations, $m_{1}=m_{3}=\mu_{31}=1$. Figure adapted from Ref. 4.

branch points are then $\sigma_{3}^{\prime \prime( \pm)}$, where

$$
\begin{aligned}
\sigma_{3}^{\prime \prime( \pm)} & =\frac{1}{2 \sigma_{1}}\left[\sigma_{1}\left(s+m_{1}^{2}+m_{3}^{2}+\mu_{13}^{2}\right)\right. \\
& +\left(m_{1}^{2}-s\right)\left(m_{3}^{2}-\mu_{13}^{2}\right)-\sigma_{1}^{2} \\
& \left. \pm \lambda^{1 / 2}\left(s, \sigma_{1}, m_{1}^{2}\right) \lambda^{1 / 2}\left(\sigma_{1}, \mu_{13}^{2}, m_{3}^{2}\right)\right],
\end{aligned}
$$

and $\sigma_{3}^{\prime \prime(-)}$ lies infinitesimally below the real axis in the physical region. Figure 5.7 shows the motion of $\sigma_{3}^{\prime \prime( \pm)}$ in the complex $\sigma_{3}^{\prime \prime}$-plane as a function of $s$ for fixed $\sigma_{1}$. At the three particle threshold, $s=\left(m_{1}+m_{2}+m_{3}\right)^{2}$, the branch points have finite imaginary part and are on opposite sides in the $\sigma_{3}^{\prime \prime}$-plane. As $s$ approaches the initial state threshold $s=\left(\sqrt{\sigma_{1}}+m_{1}\right)^{2}$, the $\sigma_{3}^{\prime \prime( \pm)}$ branch points pinch the real axis at $\sigma_{3}^{\prime \prime}=\sigma_{3}^{(a)}$. Since the $\mathcal{T}_{B}$ integration is on the real axis starting from $\sigma_{3}^{(\text {th })}$, the integration path is pinched, causing a singularity in $\mathcal{T}_{B}$ at $s=\left(\sqrt{\sigma_{1}}+m_{1}\right)$. At $\sigma_{3}^{\prime \prime}=\sigma_{3}^{(b)}$, the branch point migrates back below the real axis at a value greater than the threshold $\sigma_{3}^{(\text {th) }}$ close to the real axis. When $M^{2}>\sigma_{3}^{(b)}$, this effect generates the triangle singularity $86,94,95$. The triangle singularity has been studied as a possible mechanism to explain anomalous structures observed in heavy flavor experiments $30,96,98$. The peak of the triangle singularity coincides with the $s_{31}^{(-)}$branch point, i.e. $s_{\mathrm{tr}}=s_{31}^{(-)}$.

Aside for the unitarity branch cut starting at $s=\left(M+m_{3}\right)^{2}$ and the triangle singularity, these additional singularities in the physical $s$-plane are not allowed by analyticity. The extra 


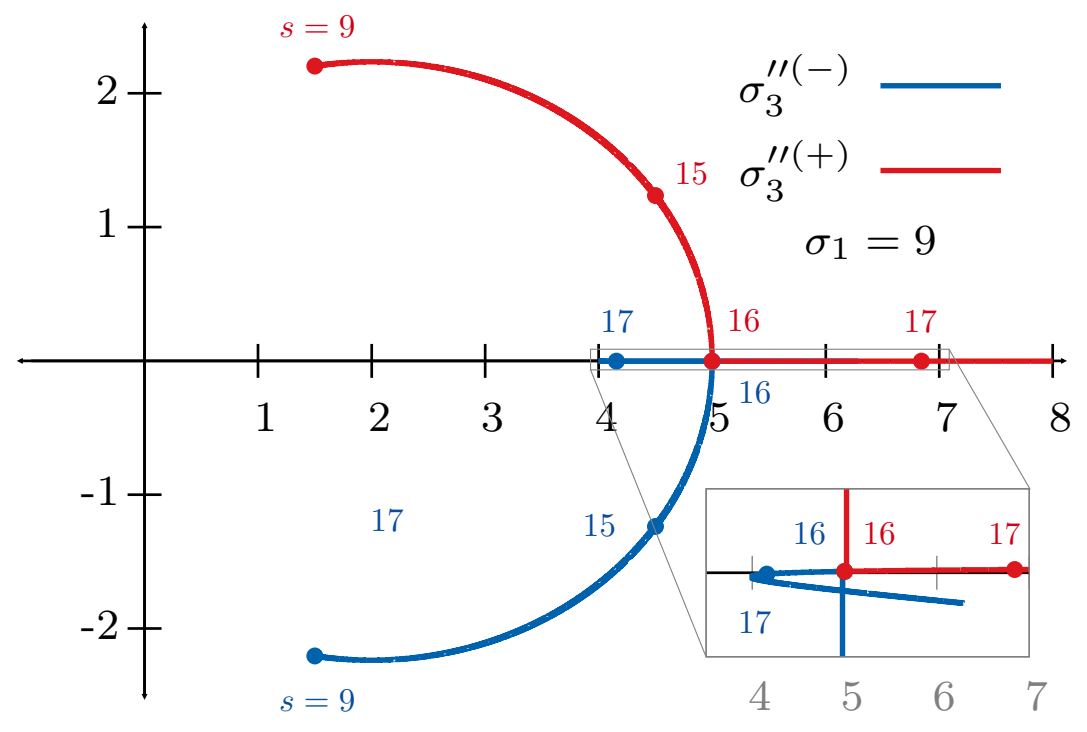

Figure 5.7: Motion of the $\sigma_{3}^{\prime \prime( \pm)}$ in the $\sigma_{3}^{\prime \prime}$-plane as a function of $s$ for fixed $\sigma_{1}$. Shown in red is $\sigma_{3}^{\prime \prime(+)}$ and in blue $\sigma_{3}^{\prime \prime(-)}$. The points indicate various $s$ values starting from the three particle threshold, $s=\left(m_{1}+m_{2}+m_{3}\right)^{2}=9$. The inset shows that the branch points pinch the real $\sigma_{1}^{\prime}$ axis at $s=\left(\sqrt{\sigma_{1}}+m_{1}\right)^{2}=16$, which is responsible for a pinch singularity in $\mathcal{T}_{B}$. Figure adapted from Ref. 4 .

singularities are moved to the second sheet when we consider the integration over the isobar shape, cf. Eq. (5.26), leaving only the unitarity cut starting at $s=\left(m_{1}+m_{2}+m_{3}\right)^{2}$ and the triangle singularity.

We compare the structure of Eq. 5.27) with that of a Feynman diagram triangle in a QFT (see Appendix E for a review of the Feynman triangle), which can be written as

$$
\mathcal{T}_{F}(s)=\int_{\Gamma_{T}} d s^{\prime} \frac{\rho_{3}\left(s^{\prime}, M^{2}\right) \widetilde{\mathcal{E}}_{13}^{S}\left(M^{2}, s^{\prime}, \sigma_{1}\right)}{s^{\prime}-s-i \epsilon},
$$

where $\Gamma_{T}$ is the path from the threshold $\left(M+m_{3}\right)^{2}$ to $\infty$, and the $S$-wave amplitudes are normalized according to Eq. 4.15). Figure 5.8 shows the real and imaginary parts, respectively, of the two triangle diagrams $\mathcal{T}_{F}$ and $\mathcal{T}_{B}$, below the region of the triangle singularity. Notice that the Feynman triangle has only a normal threshold singularity at $s=\left(M+m_{3}\right)^{2}$, and is smooth everywhere else. 

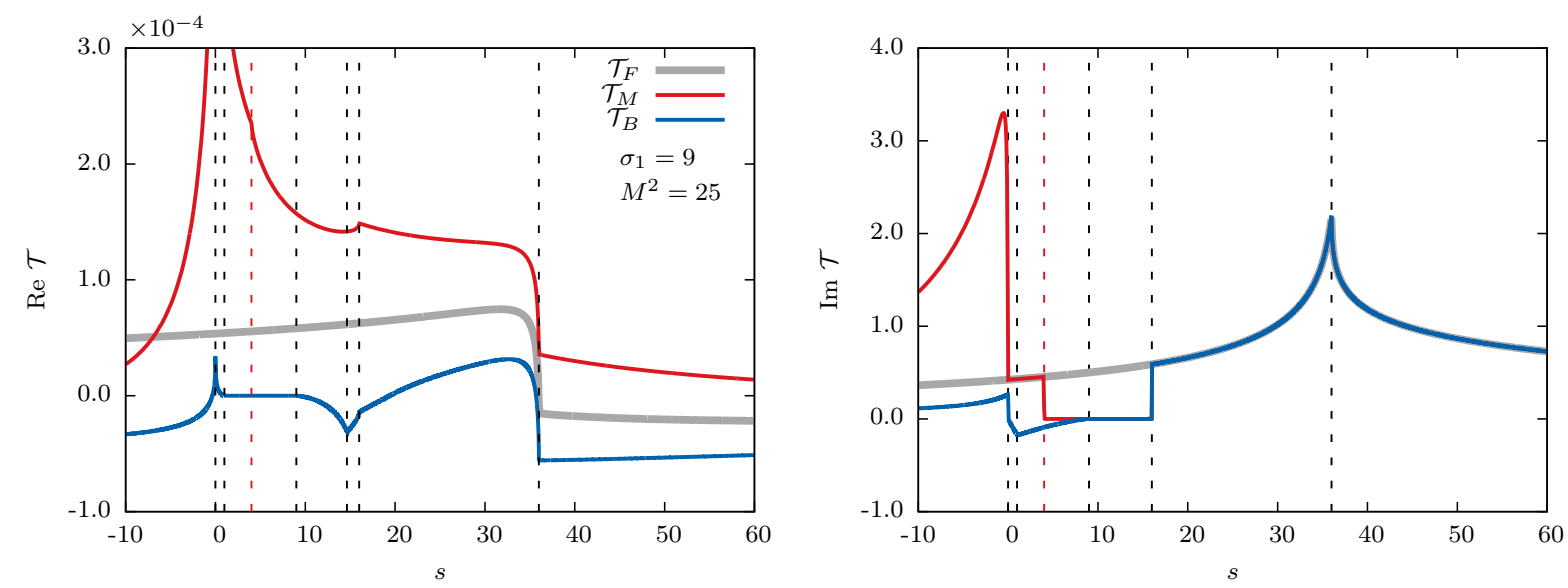

Figure 5.8: Real (left) and imaginary (right) parts of the Feynman triangle diagram, Eq. (5.29) (gray), the $B$-matrix triangle, Eq. (5.27) (blue), and the Mai et al. triangle, Eq. (5.30) (red). The external masses are set to unity, and the external isobar masses are $\sigma_{1}^{\prime}=9$ and $M^{2}=25$. The dashed vertical lines indicate the locations of singularities in the $B$-matrix as described in the text: (from left to right) the $s=0$ singularity (where the explicit $1 / s$ pole in $\rho_{3}\left(s, \sigma_{3}^{\prime \prime}\right)$ makes $\mathcal{T}_{B}$ and $\mathcal{T}_{M}$ diverge $), s=\left(\sqrt{\sigma_{3}^{(\mathrm{th})}}-m_{3}\right)^{2}=1\left(\mathcal{T}_{B}\right.$ is singular and $\mathcal{T}_{M}$ is regular $), s=\left(\sqrt{\sigma_{3}^{(\mathrm{th})}}+m_{3}\right)^{2}=9$, the crossing of the RPE cut at $s=s_{31}^{(0)}=14.64$, the initial state threshold $s=\left(\sqrt{\sigma_{1}}+m_{1}\right)=16$, and the normal threshold singularity at $s=\left(M+m_{3}\right)^{2}=36$. The red dashed line indicates the pinch singularity at $s=\left(\sqrt{\sigma_{1}}-m_{1}\right)^{2}=4$ that occurs only in $\mathcal{T}_{M}$. Figure adapted from Ref. [4].

The imaginary parts of both triangles are identical above threshold, as required by unitarity. The $B$-matrix triangle has noticeable kinks in both the real and imaginary parts below threshold, corresponding to the singularities discussed above. The black dashed lines indicate the location of the singularities. Starting from low energy, the first additional singularity is the $s=0$ singularity from the phase space. The next two singularities occur at $s=\left(\sqrt{\sigma_{3}^{(\mathrm{th})}}-m_{3}\right)^{2}$ and $\left(\sqrt{\sigma_{3}^{(\mathrm{th})}}+m_{3}\right)^{2}$, which are from the phase space evaluated at the lower integration limit. The real part contains a singularity from evaluating the OPE across the RPE cut. Note that the imaginary part does not contain this jump, consistent with the OPE description in the previous section. The next singularity occurs at the initial state threshold $s=\left(\sqrt{\sigma_{1}}+m_{1}\right)^{2}$, which is due to the pinching 


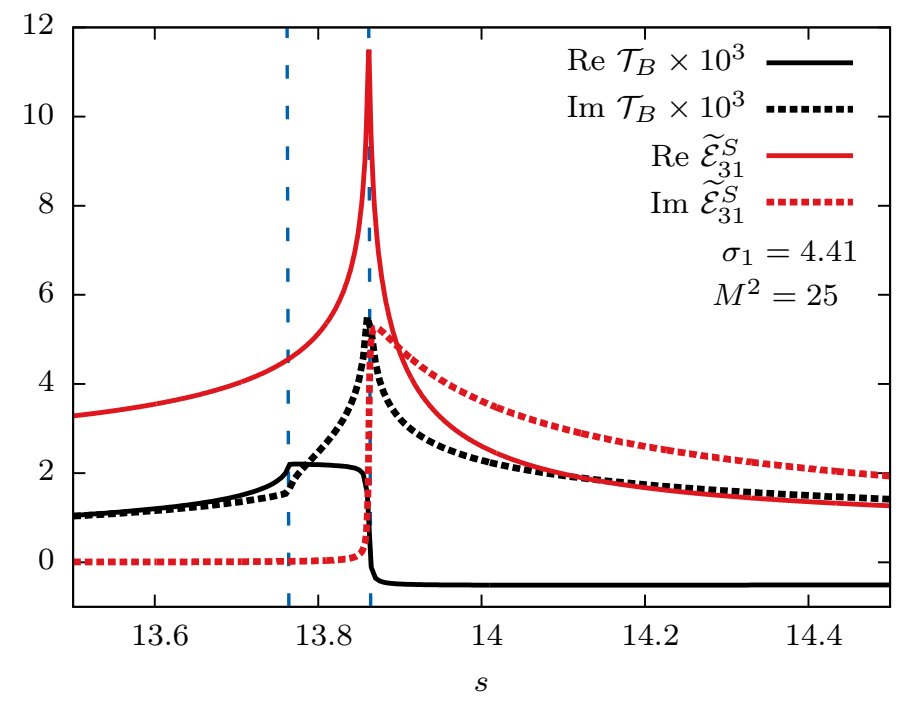

Figure 5.9: The $B$-matrix triangle Eq. (5.27) in with the triangle singularity. Shown in black are the real (solid) and imaginary (dashed) parts evaluated at $\sigma_{1}=4.41$ and $M^{2}=4.41$. Shown in red are the real (solid) and imaginary (dashed) parts of the OPE piece of the triangle, Eq. (5.17), where $\sigma_{3}^{\prime \prime}=M^{2}$. The blue dashed lines indicate the threshold $\left(M+m_{3}\right)^{2}=13.7641$ and the lower RPE branch point at $s_{31}^{(-)}=s_{\mathrm{tr}}=13.8619$. The normal threshold accounts for the first peak in the triangle diagram, while the second peak is caused by the triangle singularity. Note we scaled the triangle diagram to account for the phase space normalization of the triangle. Figure adapted from Ref. 4].

of the $\sigma_{3}^{\prime \prime}$ contours by the OPE branch points. Finally the normal threshold at $s=\left(M+m_{3}\right)^{2}$. Figure 5.9 shows $\mathcal{T}_{B}$ and the OPE in the region where the triangle singularity develops. The line shape shows the production threshold at $\left(M+m_{3}\right)^{2}$, and the peak at $s=s_{\mathrm{tr}}$. The OPE branch point $s_{31}^{(-)}$clearly coincides with the triangle peak.

Figure 5.10 shows the $\mathcal{T}_{B}$ and $\mathcal{T}_{F}$ as a function of $s$ at fixed $\sigma_{1}$ and varying $M^{2}$ in the region below and above $M^{2}=\sigma_{3}^{(b)}$. Figure 5.10(a) shows the real parts, and Fig. 5.10(b) shows the imaginary parts. At $M^{2}=\sigma_{3}^{(b)}$, the triangle singularity develops, corresponding to when $s=$ $s_{31}^{(-)}=s_{\text {tr }}$. One can see a second threshold in the line shape above threshold $\left(M+m_{3}\right)^{2}$. 

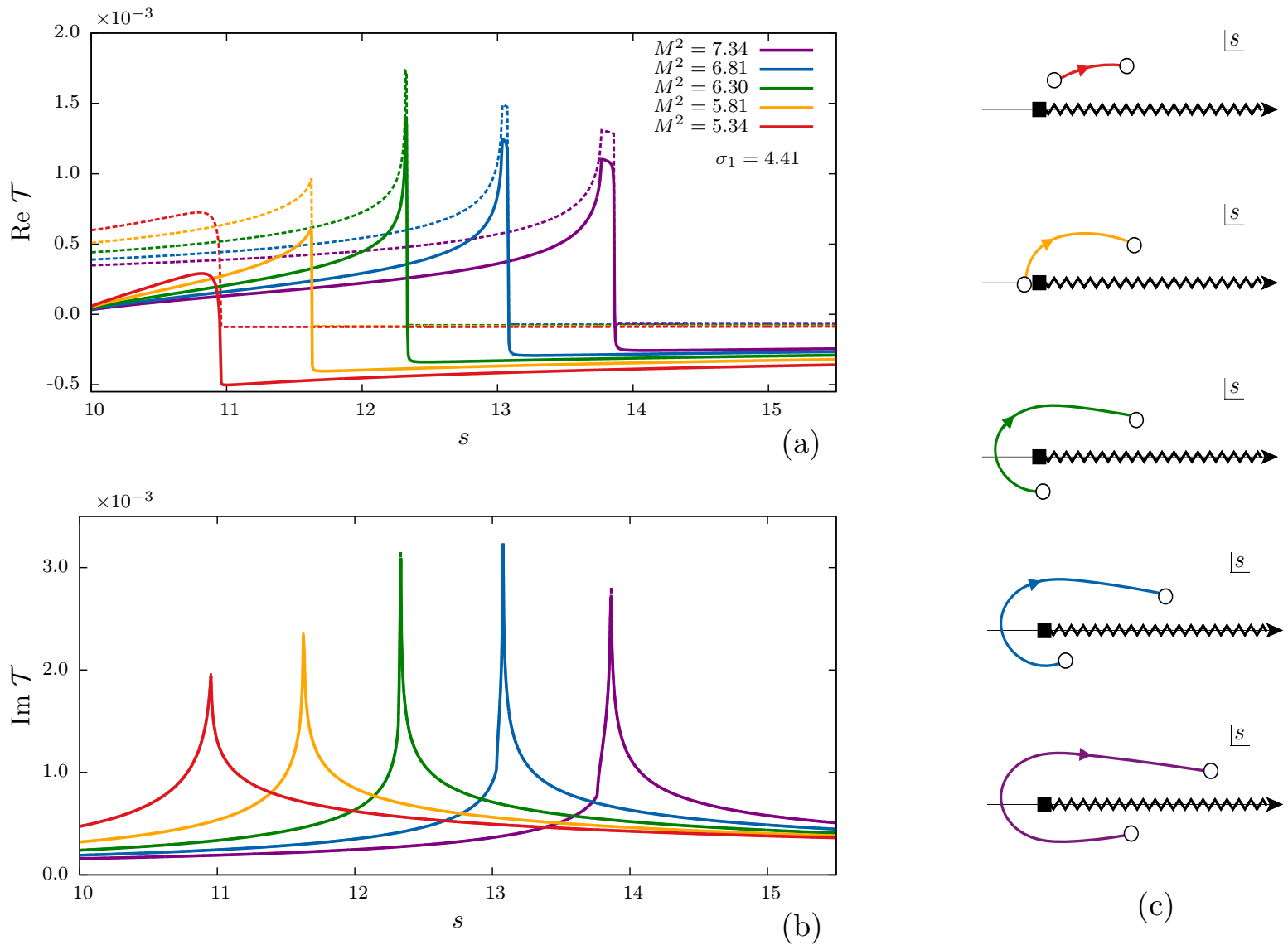

(c)

Figure 5.10: The Feynman triangle Eq. (5.29) and the $B$-matrix triangle Eq. (5.27) in the vicinity of the triangle singularity for fixed $\sigma_{1}=4.41$ and varying $M^{2}$ in the range $5.33 \leq M^{2} \leq 7.34$. $\mathcal{T}_{B}$ is shown with solid lines, $\mathcal{T}_{F}$ is shown with dashed lines. Real parts are shown in (a) and imaginary parts in (b). The imaginary parts for $\mathcal{T}_{F}$ and $\mathcal{T}_{B}$ coincide in this region. For $\sigma_{1}=4.41$, the triangle singularity region begins at $M^{2}=\sigma_{3}^{(b)}=5.81$, which manifest as a second threshold in the line shape beginning at $s=s_{31}^{(-)}=s_{\mathrm{tr}}=11.63$. The corresponding orientation of the OPE branch points are illustrated in (c). Figure adapted from Ref. [4]. 
Figure 5.10(c) shows the RPE cut of the OPE in the $s$-plane at the corresponding values for the triangle amplitude.

We also compare the $B$-matrix triangle with the analogous one from Maiet al. [84], that we denote as $\mathcal{T}_{M}$,

$$
\mathcal{T}_{M}(s)=\int_{-\infty}^{\left(\sqrt{s}-m_{3}\right)^{2}} d \sigma_{3}^{\prime \prime} \frac{\rho_{3}\left(s, \sigma_{3}^{\prime \prime}\right) \widetilde{\mathcal{E}}_{31}^{S}\left(\sigma_{3}^{\prime \prime}, s, \sigma_{1}\right)}{M^{2}-\sigma_{3}^{\prime \prime}-i \epsilon}
$$

where we take their contact term equal to unity, and the lower integration limit in their model accounts for the physics in the unphysical region. As $\sigma_{3}^{\prime \prime} \rightarrow-\infty$, the OPE amplitude goes like $1 / \sigma_{3}^{\prime \prime}$, while the phase space grows as $\sigma_{3}^{\prime \prime}$, thus the integrand goes like $1 / \sigma_{3}^{\prime \prime}$ and the function is logarithmically divergent. Numerically, we choose to cut off the integral at some large value, e.g. -200 to investigate the behavior. For $\mathcal{T}_{M}$, all lower limit endpoint singularities in $s$ from the phase space and OPE are moved toward $-\infty$. The $s=0$ pole from the phase space persist, and the normal threshold singularity at $s=\left(M+m_{3}\right)^{2}$ is present since it is from the upper limit. The pinch singularity at $s=\left(\sqrt{\sigma_{1}}+m_{1}\right)^{2}$ is also present, as well as the pinch singularity at $s=\left(\sqrt{\sigma_{1}}-m_{1}\right)^{2}$, cf. Fig. 5.7. The second pinch singularity occurs when the integration over $\sigma_{3}^{\prime \prime}$ hits $\sigma_{3}^{\prime \prime}=\sigma_{3}^{(c)}$, where

$$
\sigma_{3}^{(c)}=m_{1}^{2}+\mu_{31}^{2}-\frac{m_{1}\left(\sigma_{1}+\mu_{31}^{2}-m_{3}^{2}\right)}{\sqrt{\sigma_{1}}},
$$

is a third inflection point in the unphysical region, occurring at $s$ is at the threshold $s=\left(\sqrt{\sigma_{1}}-m_{1}\right)^{2}$ (when $\operatorname{Im} s_{k j}^{(-)}=0$ ). This pinch singularity is absent in the $B$-matrix triangle, as the integral is only over the physical region. Figure 5.8 compares the line shapes of all three triangles, $\mathcal{T}_{B}, \mathcal{T}_{F}$, and $\mathcal{T}_{M}$. Although $\mathcal{T}_{M}$ has a logarithmic divergence, we fix the lower integration limit to -200 . We see how the line shape below threshold smooths out except at the remaining singularities, shown with the black dashed lines. The red dashed line indicates the second pinch singularity in $\mathcal{T}_{M}$.

The Feynman triangle can be recovered from $\mathcal{T}_{M}$ with the method discussed by Aitchison and Pasquier 81, where the isobar approximation for $\mathbf{1} \rightarrow \mathbf{3}$ decays was studied. Using their inversion technique, it was found that the Feynman triangle can be written as a dispersive integral over the isobar invariant mass as in Ref. [84, plus additional terms. The latter are real in the physical 
region, but cure the below threshold singularities shown in the $B$-matrix. The additional terms also cancel the logarithmic divergence, leaving a finite amplitude. The addition of terms which cancel the unphysical singularities can be traced to what is chosen for $\mathcal{R}$. The $R$-matrix has the freedom to absorb these differences as they modify the structure below threshold, leaving the physical region unchanged.

\subsubsection{REMOVAL OF UNPHYSICAL SINGULARITIES}

As shown, the $B$-matrix parameterization contains additional singularities which do not match the expected analytic behavior of the amplitudes. This happens in both our formulation and Maiet al. [84]. The formalism only considers unitarity, which constrains the singularities in the physical region. Imposing additional constraints from analyticity would in principle remove these extraneous singularities, as in the $\mathbf{2} \rightarrow \mathbf{2}$ case. One possible venue for improving that is to substitute the $B$-matrix kernels, Eq. (5.13), with the Feynman one. This is in the same spirit of the Chew-Mandelstam phase space in the $\mathbf{2} \rightarrow \mathbf{2}$ parameterizations, which removes the unphysical singularities of the phase space. Although the kernels will now have the proper analytic structure (no physical sheet singularities except for the unitarity cut), the resulting amplitude will still contain singularities from iterating the kernel. Consider the solution for $\widetilde{\mathcal{A}}_{33}$ in Eq. 5.12b), where the kernel is replaced by the Feynman one, $\mathcal{K}_{33} \rightarrow \mathcal{K}_{33}^{F}$, where

$$
\mathcal{K}_{33}^{F}(s)=\int_{\text {th }}^{\infty} d s^{\prime} \frac{\widetilde{\mathcal{B}}_{31}\left(s^{\prime}\right) \rho_{3}\left(s^{\prime}\right) \widetilde{\mathcal{B}}_{13}\left(s^{\prime}\right)}{s^{\prime}-s-i \epsilon} .
$$

Now expand the solution Eq. (5.12b) in an infinite series,

$$
\widetilde{\mathcal{A}}_{33}=\mathcal{K}_{33}^{F}(s)+\mathcal{K}_{33}^{F}(s) \tau_{3}(s) \mathcal{K}_{33}^{F}(s)+\cdots
$$

The first term is the kernel, composed of Feynman diagrams which have the correct analytic properties. Let the kernel consist only of the triangle diagram, then the second term is two Feynman triangles joined with a $\tau$-function. The equivalent Feynman diagram would have two exchanges integrated over the four-momenta, which is not equivalent to what is shown in Eq. (5.33) due to the 
$\tau$-function. This diagram, as well as the higher-order ones, contain non-analyticities in a similar manner to what was shown for the triangle diagram. The unintegrated singularities from the phase space are always present. Therefore the simple kernel substitution does not produce the correct analytic behavior in the $B$-matrix solution. However, it can still be advantageous, as it corrects some of the unphysical singularities in the present $B$-matrix solution.

The remaining singularities should disappear if one was to solve the proper Bethe-Salpeter equations of the underlying QFT. The $B$-matrix parameterization is indeed reminiscent of that for $\mathbf{2} \rightarrow \mathbf{2}$ scattering. We examine some differences between these formalisms. The $B$-matrix parameterization is a covariant integral equation for the on-shell isobar-spectator amplitudes. It satisfies unitarity relations and does not have additional imposed constraints from analyticity. Thus, for complex energies on the physical Riemann sheet, the $B$-matrix parameterization contains the unitarity cut, and has additional $s$-singularities from the $\tau$ and OPE.

The Bethe-Salpeter equation is a covariant integral equations that incorporate an infinite number of exchanges for any given QFT [74. Solving it amounts to summation of exchange diagrams, similarly to the $B$-matrix. The resulting amplitudes are analytic functions in the complex $s$-plane, as the QFT amplitudes inherently obey analyticity constraints. The physical sheet thus has only the allowed singularities, such as the unitarity cut and possible bound state poles. LippmannScwhinger equations are nonrelativistic equations for the scattering amplitude in a given potential model. The $B$-matrix has similarities to the Lippmann-Schwinger equations in that both involve in a three-dimensional integral over the momenta 84 . In this work however, we focus on the physical region, and truncate the isobar mass integration appropriately. Conversely, the Bethe-Salpeter equation contains integrations over four-momenta, which results in integrating over the off-shell behavior of the amplitude. Introducing dispersive integrations in the $B$-matrix amounts to the same procedure, and would remove the unphysical singularities. 


\section{Chapter 6}

\section{EQUIVALENCE OF THREE-BODY FORMALISMS}

In finite volume studies for lattice QCD, substantial progress has been made to understand the connection between discrete energy levels and properties of hadron scattering amplitudes [40 50]. In the case of $\mathbf{2} \rightarrow \mathbf{2}$ scattering the two-particle finite volume spectrum constrains the values of the infinite volume partial wave amplitudes via the Lüscher quantization condition [9]. The multi-variable nature of $\mathbf{3} \rightarrow \mathbf{3}$ scattering amplitudes makes the derivation of the finite volume quantization condition much more complicated and different groups have approached the problem from a different angle. For example, in Refs. 40 44 the authors introduce amplitudes labeled by subchannel spins and the spectator 3-momenta. Furthermore, ladder diagrams generated by OPE are considered independently from other interactions. This implies that partial wave projection to total spin, which is necessary if one is interested in extracting properties of three-body resonances, would be performed after resummation of the OPE ladder. On the other hand, in Refs. [45, 46], the quantization conditions are derived starting from a set of amplitudes projected onto the total and subchannel spins from the start, i.e. before OPE resummation, in a spirit close to our work. In Refs. 47,49 the quantization conditions are derived in a nonrelativistic EFT framework, and the direct comparison with our $S$-matrix approach is more complicated. It is more interesting to discuss the differences with Refs. 40 43. Since we do not aim to address the subtleties of the finite volume here, we compare with the infinite volume equations derived in there on the basis of the finite volume formalism. For simplicity we ignore coupling to the 2-body channel. In Refs. [40 43], 
the $\mathbf{3} \rightarrow \mathbf{3}$ connected amplitude is denoted by $\mathcal{M}_{33}\left(\vec{k}, \vec{k}^{\prime}\right)$ (see Eq. (112) in Ref. 43 ). It contains the resummed OPE ladder and the amputated amplitude $\mathcal{T}_{33}\left(\vec{k}, \vec{k}^{\prime}\right)$ that is generated by the kernel $\mathcal{K}_{\mathrm{df}, 33}\left(\vec{k}, \vec{k}^{\prime}\right)$, which is analogous to our driving term $\widetilde{\mathcal{R}}_{\ell_{k}^{\prime} s_{k}^{\prime} ; \ell_{j} s_{j}}^{J}\left(\sigma_{k}^{\prime}, s, \sigma_{j}\right)$. Both the OPE ladder and the amplitude $\mathcal{T}_{33}\left(\vec{k}, \vec{k}^{\prime}\right)$ are solutions of linear integral equations (see Eqs. (87) and (106) in Ref. [43]), which are analogous to our Eq. (5.2).

\subsection{CONNECTING THE $R$-MATRIX TO $\mathcal{K}_{\mathrm{df}}$}

To further illustrate the connection between our amplitudes and those of Ref. [40,43], and consider the case of three identical particles in $S$-waves. The phase space $\rho_{2}$ (Eq. 4.6) of the two particle subsystem gains a factor $1 / 2$ ! to account for their identical nature. Furthermore, we shall consider the infinite sum over all partial wave amplitudes.

The resulting unitarity relations have a similar form as in Eq. 4.19). In matrix notation, one finds

$$
\begin{aligned}
\operatorname{Im} \widetilde{\mathcal{A}} & =\operatorname{Im} \widetilde{\mathcal{E}}+\operatorname{Im} \widetilde{\mathcal{E}} \rho_{3} f \widetilde{\mathcal{A}}+\widetilde{\mathcal{A}}^{*} f^{*} \rho_{3} \operatorname{Im} \widetilde{\mathcal{E}} \\
& +\widetilde{\mathcal{A}}^{*} \rho_{3} \operatorname{Im} f \widetilde{\mathcal{A}}+\widetilde{\mathcal{A}}^{*} f^{*} \rho_{3} \operatorname{Im} \widetilde{\mathcal{E}} \rho_{3} f \widetilde{\mathcal{A}}
\end{aligned}
$$

where the matrices are in the $\sigma^{\prime}, \sigma$ space with $f$ and $\rho_{3}$ diagonal matrices. The $S$-wave projection

of the OPE is given by the symmetric matrix $\widetilde{\mathcal{E}}$, and is found by the inverse relation of Eq. (4.15) on the OPE amplitude in Eq. (5.6). It is straightforward to show that the $B$-matrix parameterization (cf. Eq. $(5.2)), \widetilde{\mathcal{A}}=\widetilde{\mathcal{B}}+\widetilde{\mathcal{B}} \rho_{3} f \widetilde{\mathcal{A}}$, i.e.

$$
\widetilde{\mathcal{A}}=\left[1-(\widetilde{\mathcal{E}}+\widetilde{\mathcal{R}}) \rho_{3} f\right]^{-1}(\widetilde{\mathcal{E}}+\widetilde{\mathcal{R}})
$$

satisfies the unitarity relation, Eq. (6.1). Moreover, after simple manipulations, Eq. (6.2) can be rewritten as

$$
\mathcal{A}=f \widetilde{\mathcal{A}} f=\mathcal{D}+\mathcal{L}\left[1-\widetilde{R} \rho_{3} \mathcal{L}\right]^{-1} \widetilde{R} \mathcal{L}^{\top}
$$

where the $\mathcal{D}$ amplitude is the ladder sum of OPE, given by

$$
\mathcal{D}=f \widetilde{\mathcal{E}} f+f \widetilde{\mathcal{E}} \rho_{3} \mathcal{D}
$$


and $\mathcal{L} \equiv f+\mathcal{D} \rho_{3}$. Finally we introduce the amplitude $\mathcal{T}$ satisfying

$$
\mathcal{T}=\widetilde{\mathcal{R}}+\widetilde{\mathcal{R}} \rho_{3} \mathcal{L} \mathcal{T}
$$

and obtain an expression closely resembling that in Refs. 40, 43,

$$
\mathcal{A}=\mathcal{D}+\mathcal{L} \mathcal{T} \mathcal{L}^{\top}
$$

The difference between Eq. (6.6) and the corresponding expression for $\mathcal{M}_{33}^{(u, u)}$ in Refs. 40,43 is in the definition of $\mathcal{L}$. In our notation, the $\mathcal{L}$ of Refs. 40,43] contains an additional $1 / 3$ constant, and the $f$ and $\mathcal{D}$ matrices contain an extra factor of $\rho_{2}$.

The extra $\rho_{2}$ factor originates from a definition of amputation. We have defined our amputated amplitudes at the level of partial waves, so that $\mathcal{A}=f \widetilde{\mathcal{A}} f$, whereas the equations of Refs. 40,43 define there amputation for the full amplitudes. This can be seen when separating the four-point Green function from the six-point green function, and placing the intermediate states on their mass shell,

$$
\begin{aligned}
\int \frac{d^{3} \mathbf{k}_{k}^{\prime}}{(2 \pi)^{3} 2 \omega_{k}^{\prime}} \int \frac{d^{3} \mathbf{k}_{j}}{(2 \pi)^{3} 2 \omega_{j}}(2 \pi) \delta\left(E_{k}^{\prime}-2 \omega_{k}^{\prime}\right) \mathcal{F}_{k}\left(\hat{\mathbf{k}}^{\prime}\right) \mathcal{A}_{k j}(2 \pi) \delta\left(E_{j}-2 \omega_{j}\right) \mathcal{F}_{j}(\hat{\mathbf{k}}) \\
\longrightarrow f_{k} \rho_{2} \mathcal{A}_{k j} \rho_{2} f_{j}
\end{aligned}
$$

Keeping track of these $\rho_{2}$ factors yields the same structure as that in Refs. 40, 43]. Finally, the $1 / 3$ factor is due to how the equations in Refs. 40, 43 consider a symmeterized $\mathcal{K}_{\mathrm{df}}$, whereas we work in a completely unsymmeterized basis. One can show that the relationship between $\widetilde{\mathcal{R}}$ and $\mathcal{K}_{\mathrm{df}}$ is

$$
\widetilde{\mathcal{R}}=\mathcal{U}\left[1-\mathcal{K}\left(i \rho_{2}-\mathcal{U}^{\top}\right)\left(\frac{1}{3}+\mathcal{L} \rho_{2}\right)\right]^{-1} \mathcal{K} \mathcal{U}^{\top},
$$

where $\mathcal{U}=i \rho_{2}+\frac{1}{3} f^{-1}-\frac{1}{3} \operatorname{Im} \widetilde{\mathcal{E}}$. It is straightforward to show that this equation is real, which is required by unitarity. Eq. 6.8 represents a resummation of diagrams, in which the symmetric treatment of final state interactions are disentangled from the rest of the amplitudes. The choice of using $\widetilde{\mathcal{R}}$ versus $\mathcal{K}_{\mathrm{df}}$ is still not understood, and may impact numerical results, which is currently under study. 
Although these analogies should be verified with care, two main differences appear. One is in the treatment of the OPE dynamics, which in Ref. 43 is resummed before projection onto the total spin and in our case the projection is done first. It is likely that these approaches will ultimately prove to be equivalent, since in practical applications only a finite number of partial waves in total spin or spectator momentum components can be kept. One can also consider our formalism in the finite volume by relating integrals over the isobar invariant mass to discrete sums. This approach would lead to a quantization condition, similarly to what was shown by Ref. [45]. It remains to be seen if a quantization condition derived in this manner is identical to that of Ref. [40]. This is an active area of research, however, outside the scope of this work, and we leave it for future studies. 


\section{Chapter 7}

\section{CONCLUSION}

We have shown that amplitudes constrained by $S$-matrix principles can describe the peripheral production process $\pi p \rightarrow \eta \pi p$ in the $2^{++}$sector. Resonance parameters were extracted and two resonances were found, corresponding to the $a_{2}$ and the first excited state, the $a_{2}^{\prime}$. This analysis serves as a template for further analyses, such as the full analysis of the $\eta^{(\prime)} \pi$ system (including the exotic $P$-wave), the $3 \pi$ system in $\pi^{-} p \rightarrow \pi^{-} \pi^{-} \pi^{+} p$, where the first sector under study is $2^{-+}$[1,93], and resonance production at GlueX 99].

The success of the two-particle resonances naturally leads us to study analytic constraints for three particle scattering processes. We have shown in this thesis a unitary representation for the elastic $\mathbf{3} \rightarrow \mathbf{3}$ scattering amplitude for spinless particles. The $\mathbf{3} \rightarrow \mathbf{3}$ amplitude was described in the isobar representation. We constructed the unitarity relations for the isobar-spectator amplitudes for general partial wave quantum numbers. For a practical use, the infinite sums are truncated, leading to the standard isobar approximation. We parameterize the isobar-spectator partial wave amplitudes with the $B$-matrix formalism, which automatically satisfies the unitarity. The $B$-matrix parameterization explicitly includes the one pion exchange as a long-range contribution required by unitarity. The short-range part is not constrained by unitarity, and it can be incorporated by a specific (model-dependent) choice of the parameterization. This gives to the framework enough freedom to incorporate QCD resonances. The approach here differs from Maiet al. [84 in that the $\mathbf{2} \rightarrow \mathbf{2}$ amplitudes required as input are only needed to be known in the physical energy regions. 
The singularities of the OPE directly impact the analytic structure of the $B$-matrix kernels, and are discussed explicitly for the triangle-like diagram. The singularities in the unphysical region of our solution differ from the Maiet al. ones, and from the Feynman diagram triangle. This results in a different value for the real part of the amplitudes in the physical region. Further studies are needed to understand how to remove unexpected singularities from the $B$-matrix. We also compared our formalism to the most recent ones discussed in the literature to extract three-body scattering amplitudes from lattice QCD. In particular, the main difference with Refs. 4044 consists in the order of how the partial wave expansion and the one particle exchange ladder summation is performed. 


\section{BIBLIOGRAPHY}

[1] A. Jackura, M. Mikhasenko, and A. Szczepaniak, "Amplitude analysis of resonant production in three pions," EPJ Web Conf., vol. 130, p. 05008, 2016.

[2] A. Jackura et al., "New analysis of $\eta \pi$ tensor resonances measured at the COMPASS experiment," Phys. Lett., vol. B779, pp. 464-472, 2017.

[3] A. Jackura, "Tensor resonances in $\eta \pi$ using COMPASS data," PoS, vol. Hadron2017, p. 035, 2018.

[4] A. Jackura, C. Fernández-Ramírez, V. Mathieu, M. Mikhasenko, J. Nys, A. Pilloni, K. Saldaña, N. Sherrill, and A. P. Szczepaniak, "Phenomenology of Relativistic $\mathbf{3} \rightarrow \mathbf{3}$ Reaction Amplitudes within the Isobar Approximation," Eur. Phys. J., vol. C79, no. 1, p. 56, 2019.

[5] A. Rodas et al., "Determination of the pole position of the lightest hybrid meson candidate," Phys. Rev. Lett., vol. 122, no. 4, p. 042002, 2019.

[6] C. Patrignani et al., "Review of Particle Physics," Chin. Phys., vol. C40, no. 10, p. 100001, 2016.

[7] H. Fritzsch, M. Gell-Mann, and H. Leutwyler, "Advantages of the Color Octet Gluon Picture," Phys. Lett., vol. 47B, pp. 365-368, 1973.

[8] S. Durr et al., "Ab-Initio Determination of Light Hadron Masses," Science, vol. 322, pp. 1224$1227,2008$.

[9] M. Lüscher, "Volume Dependence of the Energy Spectrum in Massive Quantum Field Theories. 2. Scattering States," Commun. Math. Phys., vol. 105, pp. 153-188, 1986.

[10] M. Gell-Mann, "A Schematic Model of Baryons and Mesons," Phys.Lett., vol. 8, pp. 214-215, 1964. 
[11] G. Zweig, "Fractionally charged particles and $S U_{6}$," in Symmetries in Elementary Particle Physics: International School of Physics Ettore Majorana, Erice, Italy, Aug 1964, pp. 192$234,1965$.

[12] A. Esposito, A. Pilloni, and A. D. Polosa, "Multiquark Resonances," Phys. Rept., vol. 668, pp. 1-97, 2016.

[13] S. K. Choi et al., "Observation of a narrow charmonium - like state in exclusive $B^{ \pm} \rightarrow$ $K^{ \pm} \pi^{+} \pi^{-} J / \psi$ decays," Phys. Rev. Lett., vol. 91, p. 262001, 2003.

[14] R. Aaij et al., "Determination of the X(3872) meson quantum numbers," Phys. Rev. Lett., vol. 110, p. 222001, 2013.

[15] C. Adolph et al., "Odd and even partial waves of $\eta \pi^{-}$and $\eta^{\prime} \pi^{-}$in $\pi^{-} p \rightarrow \eta^{\left({ }^{\prime}\right)} \pi^{-} p$ at 191 GeV/c," Phys. Lett., vol. B740, pp. 303-311, 2015.

[16] C. Adolph et al., "Observation of a New Narrow Axial-Vector Meson $a_{1}(1420), "$ Phys. Rev. Lett., vol. 115, no. 8, p. 082001, 2015.

[17] C. Adolph et al., "Resonance Production and $\pi \pi$ S-wave in $\pi^{-}+p \rightarrow \pi^{-} \pi^{-} \pi^{+}+p_{\text {recoil }}$ at $190 \mathrm{GeV} / c, "$ Phys. Rev., vol. D95, no. 3, p. 032004, 2017.

[18] R. Akhunzyanov et al., "Light isovector resonances in $\pi^{-} p \rightarrow \pi^{-} \pi^{-} \pi^{+} p$ at $190 \mathrm{GeV} / c, "$ Phys. Rev., vol. D98, no. 9, p. 092003, 2018.

[19] C. Bookwalter, "The Search for Exotic Mesons in $\gamma p \rightarrow \pi^{+} \pi^{+} \pi^{-} n$ with CLAS at Jefferson Lab," 2011.

[20] H. Al Ghoul et al., "Measurement of the beam asymmetry $\Sigma$ for $\pi^{0}$ and $\eta$ photoproduction on the proton at $E_{\gamma}=9 \mathrm{GeV}, "$ Phys. Rev., vol. C95, no. 4, p. 042201, 2017.

[21] R. F. Lebed, R. E. Mitchell, and E. S. Swanson, "Heavy-Quark QCD Exotica," Prog. Part. Nucl. Phys., vol. 93, pp. 143-194, 2017. 
[22] S. L. Olsen, T. Skwarnicki, and D. Zieminska, "Nonstandard heavy mesons and baryons: Experimental evidence," Rev. Mod. Phys., vol. 90, no. 1, p. 015003, 2018.

[23] E. Braaten, H. W. Hammer, and T. Mehen, "Scattering of an Ultrasoft Pion and the X(3872)," Phys. Rev., vol. D82, p. 034018, 2010.

[24] C. E. Thomas and F. E. Close, "Is X(3872) a molecule?," Phys. Rev., vol. D78, p. 034007, 2008.

[25] V. Baru, A. A. Filin, C. Hanhart, Yu. S. Kalashnikova, A. E. Kudryavtsev, and A. V. Nefediev, "Three-body $D \bar{D} \pi$ dynamics for the X(3872)," Phys. Rev., vol. D84, p. 074029, 2011.

[26] Yu. S. Kalashnikova and A. V. Nefediev, "One-pion exchange in the X(3872) revisited," Pisma Zh. Eksp. Teor. Fiz., vol. 97, pp. 76-81, 2013. [JETP Lett.97,70(2013)].

[27] X.-W. Kang and J. A. Oller, "Different pole structures in line shapes of the X(3872)," Eur. Phys. J., vol. C77, no. 6, p. 399, 2017.

[28] F.-K. Guo, C. Hanhart, U.-G. Meißner, Q. Wang, Q. Zhao, and B.-S. Zou, "Hadronic molecules," Rev. Mod. Phys., vol. 90, no. 1, p. 015004, 2018.

[29] J.-L. Basdevant and E. L. Berger, "Peak locations and relative phase of different decay modes of the $a_{1}$ axial vector resonance in diffractive production," Phys. Rev. Lett., vol. 114, no. 19, p. 192001, 2015.

[30] M. Mikhasenko, B. Ketzer, and A. Sarantsev, "Nature of the $a_{1}(1420)$," Phys. Rev., vol. D91, no. 9, p. 094015, 2015.

[31] D. J. Wilson, J. J. Dudek, R. G. Edwards, and C. E. Thomas, "Resonances in coupled $\pi K, \eta K$ scattering from lattice QCD," Phys. Rev., vol. D91, no. 5, p. 054008, 2015. 
[32] C. B. Lang, L. Leskovec, D. Mohler, and S. Prelovsek, "Vector and scalar charmonium resonances with lattice QCD," JHEP, vol. 09, p. 089, 2015.

[33] J. J. Dudek, R. G. Edwards, and D. J. Wilson, "An $a_{0}$ resonance in strongly coupled $\pi \eta$, KK scattering from lattice QCD," Phys. Rev., vol. D93, no. 9, p. 094506, 2016.

[34] R. A. Briceño, J. J. Dudek, R. G. Edwards, and D. J. Wilson, "Isoscalar $\pi \pi$ scattering and the $\sigma$ meson resonance from QCD," Phys. Rev. Lett., vol. 118, no. 2, p. 022002, 2017.

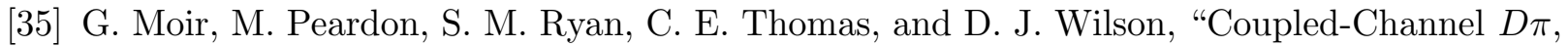
$D \eta$ and $D_{s} \bar{K}$ Scattering from Lattice QCD," JHEP, vol. 10, p. 011, 2016.

[36] R. A. Briceño, J. J. Dudek, R. G. Edwards, and D. J. Wilson, "Isoscalar $\pi \pi, K \bar{K}, \eta \eta$ scattering and the $\sigma, f_{0}, f_{2}$ mesons from QCD," Phys. Rev., vol. D97, no. 5, p. 054513, 2018.

[37] R. A. Briceño, J. J. Dudek, and R. D. Young, "Scattering processes and resonances from lattice QCD," Rev. Mod. Phys., vol. 90, p. 025001, apr 2018.

[38] A. J. Woss, C. E. Thomas, J. J. Dudek, R. G. Edwards, and D. J. Wilson, "Dynamicallycoupled partial-waves in $\rho \pi$ isospin-2 scattering from lattice QCD,” 2018.

[39] R. Brett, J. Bulava, J. Fallica, A. Hanlon, B. Hörz, and C. Morningstar, "Determination of $s$ - and $p$-wave $I=1 / 2 K \pi$ scattering amplitudes in $N_{\mathrm{f}}=2+1$ lattice QCD," Nucl. Phys., vol. B932, pp. 29-51, 2018.

[40] M. T. Hansen and S. R. Sharpe, "Relativistic, model-independent, three-particle quantization condition," Phys. Rev., vol. D90, no. 11, p. 116003, 2014.

[41] M. T. Hansen and S. R. Sharpe, "Expressing the three-particle finite-volume spectrum in terms of the three-to-three scattering amplitude," Phys. Rev., vol. D92, no. 11, p. 114509, 2015. 
[42] M. T. Hansen and S. R. Sharpe, "Applying the relativistic quantization condition to a threeparticle bound state in a periodic box," Phys. Rev., vol. D95, no. 3, p. 034501, 2017.

[43] R. A. Briceño, M. T. Hansen, and S. R. Sharpe, "Relating the finite-volume spectrum and the two-and-three-particle $S$ matrix for relativistic systems of identical scalar particles," Phys. Rev., vol. D95, no. 7, p. 074510, 2017.

[44] R. A. Briceño, M. T. Hansen, and S. R. Sharpe, "Numerical study of the relativistic threebody quantization condition in the isotropic approximation," Phys. Rev., vol. D98, no. 1, p. $014506,2018$.

[45] M. Mai and M. Döring, "Three-body Unitarity in the Finite Volume," Eur. Phys. J., vol. A53, no. 12 , p. $240,2017$.

[46] M. Mai and M. Doring, "Finite-Volume Spectrum of $\pi^{+} \pi^{+}$and $\pi^{+} \pi^{+} \pi^{+}$Systems," Phys. Rev. Lett., vol. 122, no. 6, p. 062503, 2019.

[47] K. Polejaeva and A. Rusetsky, "Three particles in a finite volume," Eur. Phys. J., vol. A48, p. $67,2012$.

[48] H. W. Hammer, J. Y. Pang, and A. Rusetsky, "Three particle quantization condition in a finite volume: 2. general formalism and the analysis of data," JHEP, vol. 10, p. 115, 2017.

[49] H. W. Hammer, J. Y. Pang, and A. Rusetsky, "Three-particle quantization condition in a finite volume: 1. The role of the three-particle force," JHEP, vol. 09, p. 109, 2017.

[50] M. Döring, H. W. Hammer, M. Mai, J. Y. Pang, A. Rusetsky, and J. Wu, "Three-body spectrum in a finite volume: the role of cubic symmetry," Phys. Rev., vol. D97, no. 11, p. $114508,2018$.

[51] L. Castillejo, R. H. Dalitz, and F. J. Dyson, "Low's scattering equation for the charged and neutral scalar theories," Phys. Rev., vol. 101, pp. 453-458, 1956. 
[52] J. D. Bjorken, "Construction of Coupled Scattering and Production Amplitudes Satisfying Analyticity and Unitarity," Phys. Rev. Lett., vol. 4, pp. 473-474, 1960.

[53] C. A. Meyer and E. S. Swanson, "Hybrid Mesons," Prog.Part.Nucl.Phys., vol. 82, pp. 21-58, 2015.

[54] W. H. Press, S. A. Teukolsky, W. T. Vetterling, and B. P. Flannery, Numerical Recipes in FORTRAN: The Art of Scientific Computing. Cambridge University Press, 1992.

[55] C. Fernández-Ramírez, I. V. Danilkin, D. M. Manley, V. Mathieu, and A. P. Szczepaniak, "Coupled-channel model for $\bar{K} N$ scattering in the resonant region," Phys.Rev., vol. D93, no. 3, p. 034029, 2016.

[56] A. N. Hiller Blin, C. Fernández-Ramírez, A. Jackura, V. Mathieu, V. I. Mokeev, A. Pilloni, and A. P. Szczepaniak, "Studying the $\mathrm{P}_{c}(4450)$ resonance in $\mathrm{J} / \psi$ photoproduction off protons," Phys. Rev., vol. D94, p. 034002, Jun 2016.

[57] J. Landay, M. Döring, C. Fernández-Ramírez, B. Hu, and R. Molina, "Model Selection for Pion Photoproduction," Phys.Rev., vol. C95, no. 1, p. 015203, 2017.

[58] K. Gottfried and J. D. Jackson, "On the Connection between production mechanism and decay of resonances at high-energies," Nuovo Cim., vol. 33, pp. 309-330, 1964.

[59] E. Byckling and K. Kajantie, Particle Kinematics. John Wiley \& Sons, 1973.

[60] V. N. Gribov, Strong Interactions of Hadrons at High Energies: Gribov lectures on. Cambridge University Press, 2012.

[61] P. Hoyer and T. L. Trueman, "Unitarity and Crossing in Reggeon-Particle Amplitudes," Phys.Rev., vol. D10, p. 921, 1974.

[62] S. U. Chung and T. L. Trueman, "Positivity Conditions on the Spin Density Matrix: A Simple Parametrization," Phys.Rev., vol. D11, p. 633, 1975. 
[63] A. Kirk et al., "New effects observed in central production by the WA102 experiment at the CERN Omega spectrometer," in 28th International Symposium on Multiparticle Dynamics (ISMD 98) Delphi, Greece, September 6-11, 1998, 1998.

[64] D. Barberis et al., "Experimental evidence for a vector like behavior of Pomeron exchange," Phys.Lett., vol. B467, pp. 165-170, 1999.

[65] D. Barberis et al., "A Study of the centrally produced $\phi \phi$ system in $p p$ interactions at 450 GeV/c," Phys.Lett., vol. B432, pp. 436-442, 1998.

[66] D. Barberis et al., "A Study of pseudoscalar states produced centrally in $p p$ interactions at $450 \mathrm{GeV} / c, "$ Phys.Lett., vol. B427, pp. 398-402, 1998.

[67] F. E. Close and A. Kirk, "A Glueball - qq filter in central hadron production," Phys.Lett., vol. B397, pp. 333-338, 1997.

[68] F. E. Close and G. A. Schuler, "Central production of mesons: Exotic states versus pomeron structure," Phys.Lett., vol. B458, pp. 127-136, 1999.

[69] T. Arens, O. Nachtmann, M. Diehl, and P. V. Landshoff, "Some tests for the helicity structure of the pomeron in ep collisions," Z.Phys., vol. C74, pp. 651-669, 1997.

[70] P. Lebiedowicz, O. Nachtmann, and A. Szczurek, "Exclusive central diffractive production of scalar and pseudoscalar mesons tensorial vs. vectorial pomeron," Annals Phys., vol. 344, pp. 301-339, 2014.

[71] P. Lebiedowicz, O. Nachtmann, and A. Szczurek, "Central exclusive diffractive production of $\pi^{+} \pi^{-}$continuum, scalar and tensor resonances in $p p$ and $p \bar{p}$ scattering within tensor pomeron approach," Phys. Rev., vol. D93, no. 5, p. 054015, 2016.

[72] C. Bromberg et al., "Study of $A_{2}$ production in the reaction $\pi^{-} p \rightarrow K^{0} K^{-} p$ at $50 \mathrm{GeV} / c$, $100 \mathrm{GeV} / c, 175 \mathrm{GeV} / c, "$ Phys.Rev., vol. D27, pp. 1-11, 1983. 
[73] C. Michael, "The production of regge recurrences," Nucl. Phys., vol. B63, pp. 431-444, 1973.

[74] C. Itzykson and J. B. Zuber, Quantum Field Theory. International Series In Pure and Applied Physics, New York: McGraw-Hill, 1980.

[75] See Supplemental material. Also on http://www.indiana.edu/ jpac/etapi-compass.php

[76] P. Lacock, C. Michael, P. Boyle, and P. Rowland, "Hybrid mesons from quenched QCD," Phys.Lett., vol. B401, pp. 308-312, 1997.

[77] C. W. Bernard et al., "Exotic mesons in quenched lattice QCD," Phys. Rev., vol. D56, pp. 7039-7051, 1997.

[78] J. J. Dudek, R. G. Edwards, P. Guo, and C. E. Thomas, "Toward the excited isoscalar meson spectrum from lattice QCD," Phys. Rev., vol. D88, no. 9, p. 094505, 2013.

[79] G. N. Fleming, "Recoupling Effects in the Isobar Model. 1. General Formalism for Three-Pion Scattering," Phys. Rev., vol. 135, pp. B551-B560, 1964.

[80] W. J. Holman, "Modified isobar approximation for $\pi$ - $\rho$ scattering," Physical Review, vol. 138, pp. B1286-B1303, jun 1965.

[81] I. J. R. Aitchison and R. Pasquier, "Three-Body Unitarity and Khuri-Treiman Amplitudes," Phys. Rev., vol. 152, no. 4, p. 1274, 1966.

[82] M. T. Grisaru, "Three-particle contributions to elastic scattering," Physical Review, vol. 146, pp. 1098-1106, jun 1966.

[83] G. Ascoli and H. W. Wyld, "Unitary States for Three Pions," Phys. Rev., vol. D12, p. 43, 1975.

[84] M. Mai, B. Hu, M. Doring, A. Pilloni, and A. Szczepaniak, "Three-body Unitarity with Isobars Revisited," Eur. Phys. J., vol. A53, no. 9, p. 177, 2017. 
[85] D. R. Giebink, "Relativity and spin in one-, two-, and three-body systems," Phys. Rev., vol. C32, pp. 502-515, 1985.

[86] R. J. Eden, P. V. Landshoff, D. I. Olive, and J. C. Polkinghorne, The Analytic S-Matrix. Cambridge: Cambridge Univ. Press, 1966.

[87] M. Tanabashi et al., "Review of Particle Physics," Phys. Rev., vol. D98, no. 3, p. 030001, 2018.

[88] G. Ascoli, L. M. Jones, B. Weinstein, and H. W. Wyld, "Partial-wave analysis of the deck amplitude for $\pi N \rightarrow \pi \pi \pi N$," Phys. Rev., vol. D8, pp. 3894-3919, 1973.

[89] F. Niecknig, B. Kubis, and S. P. Schneider, "Dispersive analysis of $\omega-\& g t ; 3 \pi$ and $\phi-\& g t ; 3 \pi$ decays," Eur. Phys. J., vol. C72, p. 2014, 2012.

[90] I. V. Danilkin, C. Fernández-Ramírez, P. Guo, V. Mathieu, D. Schott, M. Shi, and A. P. Szczepaniak, "Dispersive analysis of $\omega / \phi \rightarrow 3 \pi, \pi \gamma^{*}$," Phys. Rev., vol. D91, no. 9, p. 094029, 2015.

[91] J. L. Basdevant and E. L. Berger, "Unstable Particle Scattering and an Analytic Quasiunitary Isobar Model," Phys. Rev., vol. D19, p. 239, 1979.

[92] R. Bhandari, "QUASI TWO-BODY PHASE SPACE FACTORS IN THE ISOBAR MODEL," Phys. Rev., vol. D25, pp. 1262-1268, 1982.

[93] M. Mikhasenko, A. Jackura, B. Ketzer, and A. Szczepaniak, "Unitarity approach to the mass-dependent fit of $3 \pi$ resonance production data from the COMPASS experiment," EPJ Web Conf., vol. 137, p. 05017, 2017.

[94] R. F. Peierls, "Possible Mechanism for the Pion-Nucleon Second Resonance," Phys. Rev. Lett., vol. 6, pp. 641-643, 1961. 
[95] I. J. R. Aitchison and R. Pasquier, "Three-body unitarity and khuri-treiman amplitudes," Physical Review, vol. 152, pp. 1274-1285, dec 1966.

[96] F.-K. Guo, U.-G. Meißner, W. Wang, and Z. Yang, "How to reveal the exotic nature of the

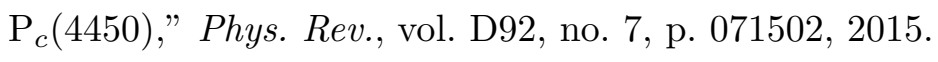

[97] F.-K. Guo, U. G. Meißner, J. Nieves, and Z. Yang, "Remarks on the $P_{c}$ structures and triangle singularities," Eur. Phys. J., vol. A52, no. 10, p. 318, 2016.

[98] A. P. Szczepaniak, "Triangle Singularities and XYZ Quarkonium Peaks," Phys. Lett., vol. B747, pp. 410-416, 2015.

[99] J. Nys, V. Mathieu, C. Fernández-Ramírez, A. Jackura, M. Mikhasenko, A. Pilloni, N. Sherrill, J. Ryckebusch, A. P. Szczepaniak, and G. Fox, "Features of $\pi \Delta$ Photoproduction at High Energies," Phys. Lett., vol. B779, pp. 77-81, 2017.

[100] D. I. Olive, "Unitarity and the evaluation of discontinuities," Il Nuovo Cimento, vol. 26, pp. $73-102$, oct 1962 . 


\section{Appendix A}

\section{KINEMATICS FOR $3 \rightarrow 3$ REACTIONS}

In this Appendix, we discuss some of the technical details of the kinematics for $\mathbf{3} \rightarrow \mathbf{3}$ processes. We first consider the system in the CMF, $\mathbf{P}^{\star}=\mathbf{P}^{\star \star}=\mathbf{0}$. The momenta in terms of invariants are

$$
\left|\mathbf{p}_{j}^{\star}\right|=\frac{\lambda^{1 / 2}\left(s, m_{j}^{2}, \sigma_{j}\right)}{2 \sqrt{s}}, \quad\left|\mathbf{p}_{k}^{\prime \star}\right|=\frac{\lambda^{1 / 2}\left(s, m_{k}^{2}, \sigma_{k}^{\prime}\right)}{2 \sqrt{s}} .
$$

Considering the particles $j$ and $k$ as spectators, then the recoiling two particles has a total momentum $\mathbf{P}_{j}^{\star}=-\mathbf{p}_{j}^{\star}$ and $\mathbf{P}_{k}^{\star \prime}=-\mathbf{p}_{k}^{\prime \star}$, for the initial and final system, respectively. The invariants $t_{j k}$ and $u_{j k}$ are related to the CMF scattering angle between spectators via

$$
\begin{aligned}
t_{j k} & =\left(p_{j}-p_{k}^{\prime}\right)^{2} \\
& =m_{j}^{2}+m_{k}^{2}-\frac{1}{2 s}\left(s+m_{j}^{2}-\sigma_{j}\right)\left(s+m_{k}^{2}-\sigma_{k}^{\prime}\right) \\
& +\frac{1}{2 s} \lambda^{1 / 2}\left(s, \sigma_{j}, m_{j}^{2}\right) \lambda^{1 / 2}\left(s, \sigma_{k}^{\prime}, m_{k}^{2}\right) z_{j k}^{\star}, \\
u_{j k} & =\left(\left(P-p_{j}\right)-p_{k}^{\prime}\right)^{2} \\
& =\sigma_{j}+m_{k}^{2}-\frac{1}{2 s}\left(s+\sigma_{j}-m_{j}^{2}\right)\left(s+m_{k}^{2}-\sigma_{k}^{\prime}\right) \\
& -\frac{1}{2 s} \lambda^{1 / 2}\left(s, \sigma_{j}, m_{j}^{2}\right) \lambda^{1 / 2}\left(s, \sigma_{k}^{\prime}, m_{k}^{2}\right) z_{j k}^{\star},
\end{aligned}
$$

where $z_{j k}^{\star}=\cos \Theta_{j k}^{\star}$. The cosine of the CMF angle between particles $j$ and $k$ is

$$
\cos \theta_{k j}^{\star}=\frac{2 s\left(\sigma_{j}+m_{k}^{2}-\mu_{j k}^{2}\right)-\left(s+\sigma_{j}-m_{j}^{2}\right)\left(s+m_{k}^{2}-\sigma_{k}\right)}{\lambda^{1 / 2}\left(s, \sigma_{j}, m_{j}^{2}\right) \lambda^{1 / 2}\left(s, \sigma_{k}, m_{k}^{2}\right)},
$$

where $\mu_{j k}$ is the mass of the particle that is neither $j$ nor $k$. 
The remaining variables needed to completely describe the $\mathbf{3} \rightarrow \mathbf{3}$ process are found by examining the IRFs. The initial $\mathrm{IRF}_{j}$ and final $\mathrm{IRF}_{k}^{\prime}$ are defined when $\mathbf{P}_{j}=\mathbf{0}$ and $\mathbf{P}_{k}^{\prime}=\mathbf{0}$, respectively. We use the convention that initial and final state variables are evaluated in their own respective IRF. The momentum of the first particle in the initial pair is denoted as $\mathbf{q}_{j}$ in the $\mathrm{IRF}_{j}$. Similarly, the first particle in the final pair is $\mathbf{q}_{k}^{\prime}$ in the $\mathrm{IRF}_{k}^{\prime}$. For example, for the final spectator 3 in the $\mathrm{IRF}_{3}^{\prime}, \mathbf{q}_{3}^{\prime}$ is the final momentum of particle 1 , and in the $\mathrm{IRF}_{1}$ of spectator $1, \mathbf{q}_{1}$ is the initial momentum of particle 3 . In terms of invariants, these momenta are

$$
\left|\mathbf{q}_{3}^{\prime}\right|=\frac{\lambda^{1 / 2}\left(\sigma_{3}^{\prime}, m_{1}^{2}, m_{2}^{2}\right)}{2 \sqrt{\sigma_{3}^{\prime}}},\left|\mathbf{q}_{1}\right|=\frac{\lambda^{1 / 2}\left(\sigma_{1}, m_{3}^{2}, m_{2}^{2}\right)}{2 \sqrt{\sigma_{1}}}
$$

The spectator momenta in these frames are

$$
\left|\mathbf{p}_{3}^{\prime}\right|=\frac{\lambda^{1 / 2}\left(s, \sigma_{3}^{\prime}, m_{3}^{2}\right)}{2 \sqrt{\sigma_{3}^{\prime}}}
$$

for the final state and

$$
\left|\mathbf{p}_{1}\right|=\frac{\lambda^{1 / 2}\left(s, \sigma_{1}, m_{1}^{2}\right)}{2 \sqrt{\sigma_{1}}}
$$

for the initial state. The helicity angles of the first particle in the IRFs are given by $\chi_{j}$ and $\chi_{k}^{\prime}$, for the initial and final states, respectively. The helicity angles are defined w.r.t. the opposite line-of-flight of the spectator. The azimuthal angles for the initial and final state are $\gamma_{j}$ and $\gamma_{k}^{\prime}$, respectively. The azimuthal angles are defined as the angle between the plane of the two particles in the CMF, and the IRFs, cf. Fig. 4.3. Note that the azimuthal angles $\gamma_{j}$ and $\gamma_{k}^{\prime}$ are invariant with respect to the Lorentz boost between the IRFs and CMF, so $\gamma_{j}=\gamma_{j}^{\star}$ and $\gamma_{k}^{\prime}=\gamma_{k}^{\prime \star}$.

The invariant masses of the other two pairs in their respective frames are related to the helicity 
angles. For example, in the $\mathrm{IRF}_{3}^{\prime}$,

$$
\begin{aligned}
\sigma_{1}^{\prime} & =\left(P-p_{1}^{\prime}\right)^{2} \\
& =s+m_{1}^{2}-\frac{1}{2 \sigma_{3}^{\prime}}\left(s+\sigma_{3}^{\prime}-m_{3}^{2}\right)\left(\sigma_{3}^{\prime}+m_{1}^{2}-m_{2}^{2}\right) \\
& +\frac{1}{2 \sigma_{3}^{\prime}} \lambda^{1 / 2}\left(s, \sigma_{3}^{\prime}, m_{3}^{2}\right) \lambda^{1 / 2}\left(\sigma_{3}^{\prime}, m_{1}^{2}, m_{2}^{2}\right) \cos \chi_{3}^{\prime}, \\
\sigma_{2}^{\prime} & =\left(P-p_{2}^{\prime}\right)^{2} \\
& =s+m_{2}^{2}-\frac{1}{2 \sigma_{3}^{\prime}}\left(s+\sigma_{3}^{\prime}-m_{3}^{2}\right)\left(\sigma_{3}^{\prime}+m_{2}^{2}-m_{1}^{2}\right) \\
& -\frac{1}{2 \sigma_{3}^{\prime}} \lambda^{1 / 2}\left(s, \sigma_{3}^{\prime}, m_{3}^{2}\right) \lambda^{1 / 2}\left(\sigma_{3}^{\prime}, m_{1}^{2}, m_{2}^{2}\right) \cos \chi_{3}^{\prime} .
\end{aligned}
$$




\section{Appendix B}

\section{DERIVATION OF $3 \rightarrow 3$ UNITARITY RELATIONS}

In this appendix we derive the general elastic unitarity relations for the $\mathbf{3} \rightarrow \mathbf{3}$ elastic scattering of distinguishable spinless particles $[79,80,86]$. For convenience, in this section we adopt the notation that the normalization of a single particle state is $\left\langle\mathbf{p}_{k}^{\prime} \mid \mathbf{p}_{j}\right\rangle=(2 \pi)^{3} 2 \omega_{j} \delta^{(3)}\left(\mathbf{p}_{k}^{\prime}-\mathbf{p}_{j}\right) \delta_{j k} \equiv$ $\widetilde{\delta}\left(p_{k}^{\prime}-p_{j}\right) \delta_{j k}$, and the invariant measure is $\widetilde{d} p_{j} \equiv d^{3} \mathbf{p}_{j} /(2 \pi)^{3} 2 \omega_{j}$. The $S$-matrix is a unitary operator, $S^{\dagger} S=\mathbf{1}$, which implies that $T-T^{\dagger}=i T^{\dagger} T$, where $S=\mathbf{1}+i T$. We consider the system in an energy range above the three particle threshold, but below the first inelastic threshold, $s_{\text {th }} \leq s<s_{\text {inel }}$. Taking matrix elements of this operator between initial and final states $|\mathbf{p}\rangle$ and $\left|\mathbf{p}^{\prime}\right\rangle$, and inserting the completeness relation $\mathbf{1}=\int \widetilde{d} p_{1}^{\prime \prime} \widetilde{d} p_{2}^{\prime \prime} \widetilde{d} p_{3}^{\prime \prime}\left|\mathbf{p}^{\prime \prime}\right\rangle\left\langle\mathbf{p}^{\prime \prime}\right|$, gives the unitarity relation

$$
\left\langle\mathbf{p}^{\prime}|T| \mathbf{p}\right\rangle-\left\langle\mathbf{p}^{\prime}\left|T^{\dagger}\right| \mathbf{p}\right\rangle=i \int \widetilde{d} p_{1}^{\prime \prime} \widetilde{d} p_{2}^{\prime \prime} \widetilde{d} p_{3}^{\prime \prime}\left\langle\mathbf{p}^{\prime}\left|T^{\dagger}\right| \mathbf{p}^{\prime \prime}\right\rangle\left\langle\mathbf{p}^{\prime \prime}|T| \mathbf{p}\right\rangle
$$

Since $T=T_{d}+T_{c}$, where $T_{d}=\sum_{j} \mathbf{1}_{j} \otimes T^{(j)}$, then the matrix element is

$$
\left\langle\mathbf{p}^{\prime}|T| \mathbf{p}\right\rangle=\left\langle\mathbf{p}^{\prime}\left|T_{c}\right| \mathbf{p}\right\rangle+\sum_{j} \widetilde{\delta}\left(p_{j}^{\prime}-p_{j}\right)\left\langle\mathbf{p}^{\prime}\left|T^{(j)}\right| \mathbf{p}\right\rangle
$$

The matrix elements $\left\langle\mathbf{p}^{\prime}\left|T^{\dagger}\right| \mathbf{p}\right\rangle$ are equal to $\left\langle\mathbf{p}^{\prime}|T| \mathbf{p}\right\rangle^{*}$ by the property of Hermitian analyticity [86, 100]. Thus the left hand side of Eq. (B.1) gives the imaginary part of the matrix element,

$$
\mathrm{LHS}=2 i \operatorname{Im}\left\langle\mathbf{p}^{\prime}\left|T_{c}\right| \mathbf{p}\right\rangle+\sum_{j} \widetilde{\delta}\left(p_{j}^{\prime}-p_{j}\right) 2 i \operatorname{Im}\left\langle\mathbf{p}^{\prime}\left|T^{(j)}\right| \mathbf{p}\right\rangle
$$

The right hand side of Eq. (B.1) is evaluated by substituting Eq. (B.2) and expanding the product into four terms, 


$$
\begin{aligned}
\mathrm{RHS}=i \int \widetilde{d} p_{1}^{\prime \prime} \widetilde{d} p_{2}^{\prime \prime} \widetilde{d p}_{3}^{\prime \prime} & {\left[\left\langle\mathbf{p}^{\prime}\left|T_{c}^{\dagger}\right| \mathbf{p}^{\prime \prime}\right\rangle\left\langle\mathbf{p}^{\prime \prime}\left|T_{c}\right| \mathbf{p}\right\rangle\right.} \\
& +\sum_{k} \widetilde{\delta}\left(p_{k}^{\prime \prime}-p_{k}^{\prime}\right)\left\langle\mathbf{p}^{\prime}\left|T^{(k) \dagger}\right| \mathbf{p}^{\prime \prime}\right\rangle\left\langle\mathbf{p}^{\prime \prime}\left|T_{c}\right| \mathbf{p}\right\rangle \\
& +\sum_{j} \widetilde{\delta}\left(p_{j}^{\prime \prime}-p_{j}\right)\left\langle\mathbf{p}^{\prime}\left|T_{c}^{\dagger}\right| \mathbf{p}^{\prime \prime}\right\rangle\left\langle\mathbf{p}^{\prime \prime}\left|T^{(j)}\right| \mathbf{p}\right\rangle \\
& \left.+\sum_{j, k} \widetilde{\delta}\left(p_{k}^{\prime \prime}-p_{k}^{\prime}\right) \widetilde{\delta}\left(p_{j}^{\prime \prime}-p_{j}\right)\left\langle\mathbf{p}^{\prime}\left|T^{(k) \dagger}\right| \mathbf{p}^{\prime \prime}\right\rangle\left\langle\mathbf{p}^{\prime \prime}\left|T^{(j)}\right| \mathbf{p}\right\rangle\right] .
\end{aligned}
$$

The fourth term contains two cases, one where $j=k$, and one where $j \neq k$, so we split the sum into the two distinct terms

$$
\begin{aligned}
\sum_{j, k} \widetilde{\delta}\left(p_{k}^{\prime \prime}-p_{k}^{\prime}\right) \widetilde{\delta}\left(p_{j}^{\prime \prime}-p_{j}\right)\left\langle\mathbf{p}^{\prime}\left|T^{(k) \dagger}\right| \mathbf{p}^{\prime \prime}\right\rangle\left\langle\mathbf{p}^{\prime \prime}\left|T^{(j)}\right| \mathbf{p}\right\rangle & \\
= & \sum_{j} \widetilde{\delta}\left(p_{j}^{\prime \prime}-p_{j}^{\prime}\right) \widetilde{\delta}\left(p_{j}^{\prime \prime}-p_{j}\right)\left\langle\mathbf{p}^{\prime}\left|T^{(j) \dagger}\right| \mathbf{p}^{\prime \prime}\right\rangle\left\langle\mathbf{p}^{\prime \prime}\left|T^{(j)}\right| \mathbf{p}\right\rangle \\
& +\sum_{\substack{j, k \\
j \neq k}} \widetilde{\delta}\left(p_{k}^{\prime \prime}-p_{k}^{\prime}\right) \widetilde{\delta}\left(p_{j}^{\prime \prime}-p_{j}\right)\left\langle\mathbf{p}^{\prime}\left|T^{(k) \dagger}\right| \mathbf{p}^{\prime \prime}\right\rangle\left\langle\mathbf{p}^{\prime \prime}\left|T^{(j)}\right| \mathbf{p}\right\rangle .
\end{aligned}
$$

We can write $\widetilde{\delta}\left(p_{j}^{\prime \prime}-p_{j}^{\prime}\right) \widetilde{\delta}\left(p_{j}^{\prime \prime}-p_{j}\right)=\widetilde{\delta}\left(p_{j}^{\prime}-p_{j}\right) \widetilde{\delta}\left(p_{j}^{\prime \prime}-p_{j}\right)$ in the first term in Eq. (B.5), thus we can identify the disconnected unitarity relation as being proportional to the spectator singularity $\widetilde{\delta}\left(p_{j}^{\prime}-p_{j}\right)$,

$$
2 \operatorname{Im}\left\langle\mathbf{p}^{\prime}\left|T^{(j)}\right| \mathbf{p}\right\rangle=\int \widetilde{d} p_{j_{1}}^{\prime \prime} \widetilde{d p}_{j_{2}}^{\prime \prime}\left\langle\mathbf{p}^{\prime}\left|T^{(j) \dagger}\right| \mathbf{p}^{\prime \prime}\right\rangle\left\langle\mathbf{p}^{\prime \prime}\left|T^{(j)}\right| \mathbf{p}\right\rangle
$$

and the connected unitarity relation

$$
\begin{aligned}
2 \operatorname{Im}\left\langle\mathbf{p}^{\prime}\left|T_{c}\right| \mathbf{p}\right\rangle & =\int \widetilde{d} p_{1}^{\prime \prime} \widetilde{d} p_{2}^{\prime \prime} \widetilde{d} p_{3}^{\prime \prime}\left[\left\langle\mathbf{p}^{\prime}\left|T_{c}^{\dagger}\right| \mathbf{p}^{\prime \prime}\right\rangle\left\langle\mathbf{p}^{\prime \prime}\left|T_{c}\right| \mathbf{p}\right\rangle\right. \\
& +\sum_{k} \widetilde{\delta}\left(p_{k}^{\prime \prime}-p_{k}^{\prime}\right)\left\langle\mathbf{p}^{\prime}\left|T^{(k) \dagger}\right| \mathbf{p}^{\prime \prime}\right\rangle\left\langle\mathbf{p}^{\prime \prime}\left|T_{c}\right| \mathbf{p}\right\rangle \\
& +\sum_{j} \widetilde{\delta}\left(p_{j}^{\prime \prime}-p_{j}\right)\left\langle\mathbf{p}^{\prime}\left|T_{c}^{\dagger}\right| \mathbf{p}^{\prime \prime}\right\rangle\left\langle\mathbf{p}^{\prime \prime}\left|T^{(j)}\right| \mathbf{p}\right\rangle \\
& \left.+\sum_{\substack{j, k \\
j \neq k}} \widetilde{\delta}\left(p_{k}^{\prime \prime}-p_{k}^{\prime}\right) \widetilde{\delta}\left(p_{j}^{\prime \prime}-p_{j}\right)\left\langle\mathbf{p}^{\prime}\left|T^{(k) \dagger}\right| \mathbf{p}^{\prime \prime}\right\rangle\left\langle\mathbf{p}^{\prime \prime}\left|T^{(j)}\right| \mathbf{p}\right\rangle\right]
\end{aligned}
$$

The momenta with $j_{1}$ and $j_{2}$ in Eq. (B.6) identify the first and second particle in the pair. Substituting Eqs. (4.1) and 4.2) into Eqs. (B.6) and (B.7), and evaluating the phase space integrals 
yield the unitarity relations Eqs. 4.5 and 4.7. 


\section{Appendix C}

\section{DERIVATION OF PWIS UNITARITY RELATIONS}

Using the assumption of the isobar model Eq. (4.14), we derive a set of unitarity relations for the amputated PWIS amplitudes. Inserting Eq. (4.14) into the unitarity relations Eq. (4.7) leads to a unitarity relation for the $\mathcal{A}_{k j}$ isobar-spectator amplitude,

$$
\begin{aligned}
& \operatorname{Im} \mathcal{A}_{k j}\left(\mathbf{p}^{\prime} ; \mathbf{p}\right)=\frac{1}{\pi\left(32 \pi^{2}\right)^{2}} \sum_{n} \int_{\sigma_{n}^{(\mathrm{th})}}^{\left(\sqrt{s}-m_{n}\right)^{2}} d \sigma_{n}^{\prime \prime} \frac{\left|\mathbf{q}_{n}^{\prime \prime}\right|\left|\mathbf{p}_{n}^{\prime \prime \star}\right|}{\sqrt{\sigma_{n}^{\prime \prime} \sqrt{s}}} \int d \widehat{\mathbf{P}}_{n}^{\prime \prime \star} \int d \widehat{\mathbf{q}}_{n}^{\prime \prime} \\
& \times \mathcal{A}_{n k}^{*}\left(\mathbf{p}^{\prime \prime} ; \mathbf{p}^{\prime}\right) \mathcal{A}_{n j}\left(\mathbf{p}^{\prime \prime} ; \mathbf{p}\right) \Theta\left(s-s_{\mathrm{th}}\right) \\
& +\frac{1}{\pi\left(32 \pi^{2}\right)^{2}} \sum_{\substack{n, r \\
n \neq r}} \int_{\sigma_{n}^{(\mathrm{th})}}^{\left(\sqrt{s}-m_{n}\right)^{2}} d \sigma_{n}^{\prime \prime} \frac{\left|\mathbf{q}_{n}^{\prime \prime} \| \mathbf{p}_{n}^{\prime \prime \star}\right|}{\sqrt{\sigma_{n}^{\prime \prime}} \sqrt{s}} \int d \widehat{\mathbf{P}}_{n}^{\prime \prime \star} \int d \widehat{\mathbf{q}}_{n}^{\prime \prime} \\
& \times \mathcal{A}_{r k}^{*}\left(\mathbf{p}^{\prime \prime} ; \mathbf{p}^{\prime}\right) \mathcal{A}_{n j}\left(\mathbf{p}^{\prime \prime} ; \mathbf{p}\right) \Theta\left(s-s_{\mathrm{th}}\right) \\
& +\left.\rho_{2}\left(\sigma_{k}^{\prime}\right) \int d \widehat{\mathbf{q}}_{k}^{\prime \prime} \mathcal{F}_{k}^{*}\left(\mathbf{p}^{\prime \prime} ; \mathbf{p}^{\prime}\right) \mathcal{A}_{k j}\left(\mathbf{p}^{\prime \prime} ; \mathbf{p}\right)\right|_{\mathbf{p}_{k}^{\prime \prime}=\mathbf{p}_{k}^{\prime}} \Theta\left(\sigma_{k}^{\prime}-\sigma_{k}^{(\mathrm{th})}\right) \\
& +\left.\rho_{2}\left(\sigma_{j}\right) \int d \widehat{\mathbf{q}}_{j}^{\prime \prime} \mathcal{A}_{k j}^{*}\left(\mathbf{p}^{\prime \prime} ; \mathbf{p}^{\prime}\right)\right|_{\mathbf{p}_{j}^{\prime \prime}=\mathbf{p}_{j}} \mathcal{F}_{j}\left(\mathbf{p}^{\prime \prime} ; \mathbf{p}\right) \Theta\left(\sigma_{j}-\sigma_{j}^{(\mathrm{th})}\right) \\
& +\left.\rho_{2}\left(\sigma_{k}^{\prime}\right) \sum_{\substack{r \\
k \neq r}} \int d \widehat{\mathbf{q}}_{k}^{\prime \prime} \mathcal{F}_{k}^{*}\left(\mathbf{p}^{\prime \prime} ; \mathbf{p}^{\prime}\right) \mathcal{A}_{r j}\left(\mathbf{p}^{\prime \prime} ; \mathbf{p}\right)\right|_{\mathbf{p}_{k}^{\prime \prime}=\mathbf{p}_{k}^{\prime}} \Theta\left(\sigma_{k}^{\prime}-\sigma_{k}^{(\mathrm{th})}\right) \\
& +\left.\rho_{2}\left(\sigma_{j}\right) \sum_{\substack{n \\
n \neq j}} \int d \widehat{\mathbf{q}}_{j}^{\prime \prime} \mathcal{A}_{k n}^{*}\left(\mathbf{p}^{\prime \prime} ; \mathbf{p}^{\prime}\right)\right|_{\mathbf{p}_{j}^{\prime \prime}=\mathbf{p}_{j}} \mathcal{F}_{j}\left(\mathbf{p}^{\prime \prime} ; \mathbf{p}\right) \Theta\left(\sigma_{j}-\sigma_{j}^{(\mathrm{th})}\right) \\
& +\left.\left.\pi \delta\left(u_{j k}-\mu_{j k}^{2}\right) \mathcal{F}_{k}^{*}\left(\mathbf{p}^{\prime \prime} ; \mathbf{p}^{\prime}\right)\right|_{\mathbf{p}_{j}^{\prime \prime}=\mathbf{p}_{j}} \mathcal{F}_{j}\left(\mathbf{p}^{\prime \prime} ; \mathbf{p}\right)\right|_{\mathbf{p}_{k}^{\prime \prime}=\mathbf{p}_{k}^{\prime}}\left(1-\delta_{j k}\right),
\end{aligned}
$$

where we wrote the three-body phase space factor in the first two terms,

$$
\begin{aligned}
& \frac{1}{2(2 \pi)^{5}} \int \frac{d^{3} \mathbf{p}_{1}^{\prime \prime}}{2 \omega_{1}^{\prime \prime}} \frac{d^{3} \mathbf{p}_{2}^{\prime \prime}}{2 \omega_{2}^{\prime \prime}} \frac{d^{3} \mathbf{p}_{3}^{\prime \prime}}{2 \omega_{3}^{\prime \prime}} \delta^{(4)}\left(P^{\prime \prime}-P\right) \\
& =\frac{1}{\pi\left(32 \pi^{2}\right)^{2}} \int_{\sigma_{n}^{(\mathrm{th})}}^{\left(\sqrt{s}-m_{n}\right)^{2}} d \sigma_{n}^{\prime \prime} \frac{\left|\mathbf{q}_{n}^{\prime \prime}\right|\left|\mathbf{p}_{n}^{\prime \prime}\right|}{\sqrt{\sigma_{n}^{\prime \prime}} \sqrt{s}} \int d \widehat{\mathbf{P}}_{n}^{\prime \prime \star} \int d \widehat{\mathbf{q}}_{n}^{\prime \prime},
\end{aligned}
$$




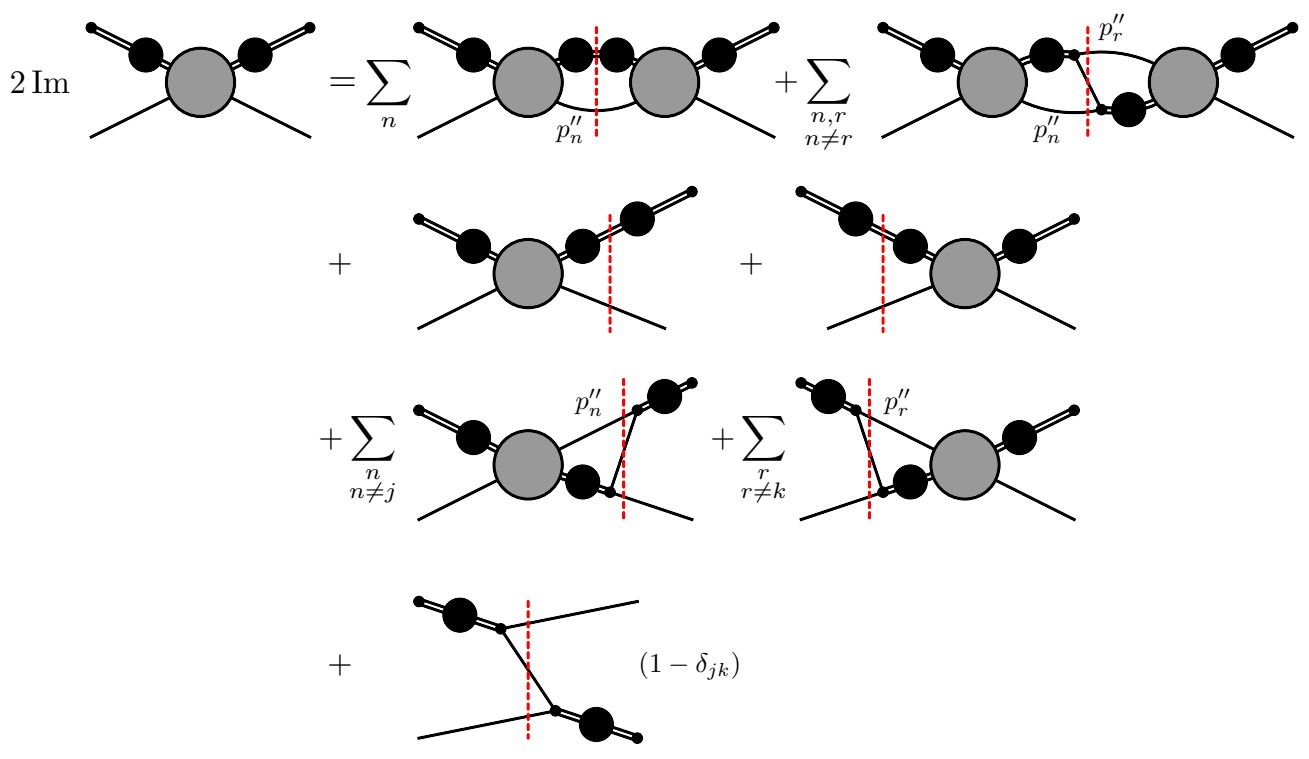

Figure C.1: Diagrammatic representation for the isobar-spectator unitarity relation in Eq. (C.1).

where $\widehat{\mathbf{P}}_{n}^{\prime \prime \star}$ is the orientation of the isobar associated with spectator $n$ in the intermediate state, and $\widehat{\mathbf{q}}_{n}^{\prime \prime}$ is the orientation of the first particle in the intermediate isobar in its rest frame. The terms in the intermediate state have been split up into two groups, diagonal and off-diagonal. The diagonal terms in Eq. (C.1) (first, third, and fourth line) are terms such that the isobar propagates in the intermediate state without breaking up. The off-diagonal terms (second, fifth, and sixth line in Eq. (C.1) ) are ones where the isobar breaks up in the intermediate state, and combines with the spectator to form a new isobar. Figure C.1 shows the diagonal and off-diagonal topologies in the intermediate state.

The partial wave expansion Eq. 4.15) can be derived by considering the expansion in three steps. The first step is to perform the expansion of the isobars into definite spin and helicity,

$$
\mathcal{A}_{k j}=\sum_{s_{k}^{\prime}, \lambda_{k}^{\prime}} \sum_{s_{j}, \lambda_{j}} Y_{\lambda_{k}^{\prime}}^{s_{k}^{\prime}}\left(\widehat{\mathbf{q}}_{k}^{\prime}\right) \mathcal{A}_{s_{k}^{\prime} \lambda_{k}^{\prime} ; s_{j} \lambda_{j}} Y_{\lambda_{j}}^{s_{j} *}\left(\widehat{\mathbf{q}}_{j}\right)
$$

where $\lambda_{j}, \lambda_{k}^{\prime}$ are defined along the direction of the isobar in the CMF. The expansion removes the $\widehat{\mathbf{q}}_{k}^{\prime}$ and $\widehat{\mathbf{q}}_{j}$ dependence in the helicity amplitude $\mathcal{A}_{s_{k}^{\prime} \lambda_{k}^{\prime} ; s_{j} \lambda_{j}}$. Second, the helicity amplitude can be 
expanded in partial waves,

$$
\mathcal{A}_{s_{k}^{\prime} \lambda_{k}^{\prime} ; s_{j} \lambda_{j}}=\sum_{J} \mathcal{N}_{J}^{2} \mathcal{A}_{s_{k}^{\prime} \lambda_{k}^{\prime} ; s_{j} \lambda_{j}}^{J}\left(\sigma_{k}^{\prime}, s, \sigma_{j}\right) \sum_{M} \mathcal{D}_{M \lambda_{k}^{\prime}}^{(J) *}\left(\widehat{\mathbf{P}}_{k}^{\prime \star}\right) \mathcal{D}_{M \lambda_{j}}^{(J)}\left(\widehat{\mathbf{P}}_{j}^{\star}\right)
$$

where $\mathcal{N}_{J}^{2}=(2 J+1) / 4 \pi$. Finally, since parity is not a good quantum number in the helicity basis, we convert the helicity partial wave amplitudes into $L S$ partial wave amplitudes,

$$
\mathcal{A}_{\ell_{k}^{\prime} s_{k}^{\prime} ; \ell_{j} s_{j}}^{J}=\sum_{\lambda_{k}^{\prime}, \lambda_{j}} C_{\ell_{k}^{\prime} s_{k}^{\prime} \lambda_{k}^{\prime}}^{J} C_{\ell_{j} s_{j} \lambda_{j}}^{J} \mathcal{A}_{s_{k}^{\prime} \lambda_{k}^{\prime} ; s_{j} \lambda_{j}}^{J}
$$

where $C_{\ell s \lambda}^{J}=\sqrt{(2 \ell+1) /(2 J+1)}\langle J \lambda \mid \ell 0 s \lambda\rangle$, and has the completeness relation

$$
\sum_{\lambda} C_{\ell s \lambda}^{J} C_{\ell^{\prime} s^{\prime} \lambda}^{J}=\delta_{J J^{\prime}} \delta_{\ell \ell^{\prime}} \delta_{s s^{\prime}}
$$

Combining Eqs. (C.3), (C.4), and (C.5) yields the partial wave expansion Eq. 4.15). We apply the expansion to Eq. (C.1) to obtain the PWIS unitarity relations. The diagonal terms are most directly evaluated using the orthonormality condition, Eq. 4.13). Since $\mathbf{p}_{k}^{\prime \prime}=\mathbf{p}_{k}^{\prime}$ in the third term, and $\mathbf{p}_{j}^{\prime \prime}=\mathbf{p}_{j}$ in the fourth term, then the intermediate isobar orientation is identical to that of the final and initial state isobar, respectively. The integrations over $\widehat{\mathbf{q}}_{k}^{\prime}$ and $\widehat{\mathbf{q}}_{j}$ can be performed by writing Eq. (4.8) using the spherical harmonic addition theorem.

The off-diagonal terms are more challenging, as they involve two different angles in the intermediate state, thus the rotational functions will not directly integrate out. We can make use of the group properties of rotations to simplify the intermediate rotational functions to a recoupling coefficient. The off-diagonal terms on the right-hand side of Eq. (C.1) under the expansion Eq. (4.15) will contain terms of the form

$$
\begin{aligned}
& \mathcal{D}_{\lambda_{n} 0}^{\left(s_{n}\right) *}\left(\widehat{\mathbf{q}}_{n}\right) \mathcal{D}_{M \lambda_{n}}^{(J) *}\left(\widehat{\mathbf{P}}_{n}^{\star}\right) \mathcal{D}_{M \lambda_{r}}^{(J)}\left(\widehat{\mathbf{P}}_{r}^{\star}\right) \mathcal{D}_{\lambda_{r} 0}^{\left(s_{r}\right)}\left(\widehat{\mathbf{q}}_{r}\right) \\
& \quad=d_{\lambda_{n} 0}^{\left(s_{n}\right)}\left(\cos \chi_{n}\right) \mathcal{D}_{M \lambda_{n}}^{(J) *}\left(R_{n}^{\star}\right) \mathcal{D}_{M \lambda_{r}}^{(J)}\left(R_{r}^{\star}\right) d_{\lambda_{r} 0}^{\left(s_{r}\right)}\left(\cos \chi_{r}\right),
\end{aligned}
$$

where $n \neq r$, and we combined the terms with $\gamma_{n}$ and the orientation of the isobar to the set of angles $R_{n}^{\star}=\left(\alpha_{n}^{\star}, \beta_{n}^{\star}, \gamma_{n}^{\star}\right)$, where $\alpha_{n}^{\star}$ is the azimuthal angle of the isobar and $\beta_{n}^{\star}$ is the polar angle, w.r.t. some fixed coordinate system. Note that since we boost along the direction of the isobar to 
go between CMF and IRFs, $\gamma_{n}=\gamma_{n}^{\star}$. The angles $R^{\star}$ are the Euler angles describing the orientation of the three particles in their CMF. Since these two sets of angles describe the same configuration of three particles, with the only difference being which particle is the spectator, the angles $R_{n}^{\star}$ and $R_{r}^{\star}$ must be related by a rotation.

Each set of angles can be found by rotating from some initial standard configuration. We define the standard configuration such that the three particle system lies in the $x z$-plane, where the spectator momenta is along the negative $z$-axis, cf. Fig. C.2. Then, the difference in the Euler angles is a rotation about the $y$-axis,

$$
R_{r}^{\star}=R_{n}^{\star} r_{n r}^{\star},
$$

where $r_{n r}^{\star}$ is the rotation relating the two standard configurations [83, 85]. Here, the rotation is about the $y$-axis, $r_{n r}^{\star}=R_{y}\left(\theta_{n r}^{\star}\right)$, where $\theta_{n r}^{\star}$ is given in Eq. A.3. Thus, the rotation is a function of the invariant masses of the isobars, $\theta_{n r}^{\star}=\theta_{n r}^{\star}\left(\sigma_{n}, s, \sigma_{r}\right)$. Note that the inverse rotation is given by $r_{r n}^{\star}=r_{n r}^{\star}-1$.

Therefore, we can relate the two Wigner $\mathcal{D}$-matrices using the group addition property

$$
\mathcal{D}_{M \lambda_{r}}^{(J)}\left(R_{r}^{\star}\right)=\sum_{\lambda_{n}} \mathcal{D}_{M \lambda_{n}}^{(J)}\left(R_{n}^{\star}\right) \mathcal{D}_{\lambda_{n} \lambda_{r}}^{(J)}\left(r_{n r}^{\star}\right)
$$

The integration over the Euler angles in the intermediate state can be performed, leaving one
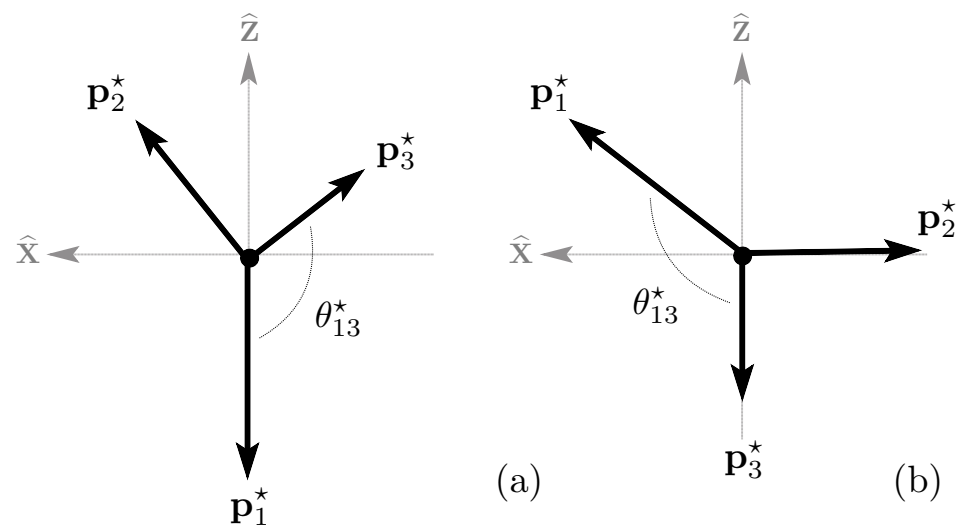

Figure C.2: The standard configurations considering (a) particle 1 as the spectator and (b) particle 3 as the spectator. 
rotation that recouples the isobars,

$$
\begin{aligned}
\int d R_{n}^{\star} & \mathcal{D}_{M \lambda_{n}}^{(J) *}\left(R_{n}^{\star}\right) \mathcal{D}_{M \lambda_{r}}^{(J)}\left(R_{r}^{\star}\right) \\
& =\sum_{\lambda} \int d R_{n}^{\star} \mathcal{D}_{M \lambda_{n}}^{(J) *}\left(R_{n}^{\star}\right) \mathcal{D}_{M \lambda}^{(J)}\left(R_{n}^{\star}\right) \mathcal{D}_{\lambda \lambda_{r}}^{(J)}\left(r_{n r}^{\star}\right) \\
& =\frac{8 \pi^{2}}{2 J+1} d_{\lambda_{n} \lambda_{r}}^{(J)}\left(\cos \theta_{n r}^{\star}\right) .
\end{aligned}
$$

where $d R_{n}^{\star}=d \alpha_{n}^{\star} d \cos \beta_{n}^{\star} d \gamma_{n}^{\star}$.

Since the angle $\chi$ can be written in terms of the invariant masses of the isobars, it is advantages to write the phase space in terms of the Dalitz variables,

$$
\begin{aligned}
& \frac{1}{(2 \pi)^{5}} \int \frac{d^{3} \mathbf{p}_{1}^{\prime \prime}}{2 \omega_{1}^{\prime \prime}} \frac{d^{3} \mathbf{p}_{2}^{\prime \prime}}{2 \omega_{2}^{\prime \prime}} \frac{d^{3} \mathbf{p}_{3}^{\prime \prime}}{2 \omega_{3}^{\prime \prime}} \delta^{(4)}\left(P^{\prime \prime}-P\right) \\
& =\frac{1}{\pi s\left(32 \pi^{2}\right)^{2}} \int_{\sigma_{n}^{(\text {th })}}^{\left(\sqrt{s}-m_{n}^{2}\right)^{2}} d \sigma_{n}^{\prime \prime} \int_{\sigma_{r}^{(-)}}^{\sigma_{r}^{(+)}} d \sigma_{r}^{\prime \prime} \int d R_{n}^{\prime \prime \star},
\end{aligned}
$$

where we used

$$
d \sigma_{r}^{\prime \prime}=2 \frac{\sqrt{s}}{\sqrt{\sigma_{n}^{\prime \prime}}}\left|\mathbf{q}_{n}^{\prime \prime}\right|\left|\mathbf{p}_{n}^{\prime \prime \star}\right| d \cos \chi_{n}^{\prime \prime}
$$

to rewrite Eq. C.2). The Dalitz region is bounded by $\sigma_{n}^{(\text {th })} \leq \sigma_{n}^{\prime \prime} \leq\left(\sqrt{s}-m_{n}\right)^{2}$ and $\sigma_{r}^{(-)} \leq \sigma_{r}^{\prime \prime} \leq$ $\sigma_{r}^{(+)}$, where $\sigma_{r}^{( \pm)}=\sigma_{r}^{( \pm)}\left(\sigma_{n}^{\prime \prime}\right)$ is found by the physical boundary $\cos \chi_{n}^{\prime \prime}= \pm 1$, e.g. for $n=1$ and $r=3$, then

$$
\begin{aligned}
\sigma_{3}^{( \pm)} & =m_{1}^{2}+m_{2}^{2}-\frac{1}{2 \sigma_{1}^{\prime \prime}}\left(\sigma_{1}^{\prime \prime}-s+m_{1}^{2}\right)\left(\sigma_{1}^{\prime \prime}+m_{2}^{2}-m_{3}^{2}\right) \\
& \pm \frac{1}{2 \sigma_{1}^{\prime \prime}} \lambda^{1 / 2}\left(s, \sigma_{1}^{\prime \prime}, m_{1}^{2}\right) \lambda^{1 / 2}\left(\sigma_{1}^{\prime \prime}, m_{2}^{2}, m_{3}^{2}\right)
\end{aligned}
$$

The last piece needed is the partial wave projection of the OPE term. To evaluate the partial wave projection, we write the delta-function as

$$
\delta\left(u_{j k}-\mu_{j k}^{2}\right)=\frac{1}{2\left|\mathbf{p}_{k}^{\prime \star}\right|\left|\mathbf{p}_{j}^{\star}\right|} \delta\left(z_{k j}^{\star}-z_{k j}\right)
$$

where $z_{k j}$ is defined in Eq. (5.18). Then, the completeness relation for the delta-function allows us to write Eq. (C.14) as

$$
\delta\left(z_{k j}^{\star}-z_{k j}\right)=\sum_{J}\left(\frac{2 J+1}{2}\right) d_{\lambda^{\prime} \lambda}^{(J)}\left(z_{k j}^{\star}\right) d_{\lambda^{\prime} \lambda}^{(J)}\left(z_{k j}\right),
$$


where $\lambda$ and $\lambda^{\prime}$ are arbitary, and thus we may choose them to align with $\lambda_{j}$ and $\lambda_{k}^{\prime}$, respectively. Then, $d_{\lambda_{j} \lambda_{k}^{\prime}}^{(J)}\left(z_{k j}^{\star}\right)$ is written in terms of the angles $\widehat{\mathbf{P}}_{j}^{\star}$ and $\widehat{\mathbf{P}}_{k}^{\prime \star}$, via the group addition property,

$$
d_{\lambda^{\prime} \lambda}^{(J)}\left(z_{k j}^{\star}\right)=\sum_{M} \mathcal{D}_{M \lambda^{\prime}}^{(J) *}\left(\widehat{\mathbf{P}}_{k}^{\prime \star}\right) \mathcal{D}_{M \lambda}^{(J)}\left(\widehat{\mathbf{P}}_{j}^{\star}\right)
$$

Finally, the $\mathbf{2} \rightarrow \mathbf{2}$ amplitudes are written using Eq. 4.8

$$
\begin{aligned}
& F_{k}^{*} F_{j}= f_{s_{k}^{\prime}}^{*}\left(\sigma_{k}^{\prime}\right) f_{s_{j}}\left(\sigma_{j}\right) P_{s_{k}^{\prime}}\left(\widehat{\overline{\mathbf{q}}}_{j} \cdot \widehat{\mathbf{q}}_{k}^{\prime}\right) P_{s_{j}}\left(\widehat{\mathbf{q}}_{j} \cdot \widehat{\overline{\mathbf{q}}}_{k}^{\prime}\right) \\
&=f_{s_{k}^{\prime}}^{*}\left(\sigma_{k}^{\prime}\right) f_{s_{j}}\left(\sigma_{j}\right) \sum_{\lambda_{k}^{\prime}} \mathcal{D}_{\lambda_{k}^{\prime} 0}^{\left(s_{k}^{\prime}\right) *}\left(\widehat{\mathbf{q}}_{k}^{\prime}\right) \mathcal{D}_{\lambda_{k}^{\prime} 0}^{\left(s_{k}^{\prime}\right)}\left(\widehat{\overline{\mathbf{q}}}_{j}\right) \\
& \quad \times \sum_{\lambda_{j}} \mathcal{D}_{\lambda_{j} 0}^{\left(s_{j}\right) *}\left(\widehat{\overline{\mathbf{q}}}_{k}^{\prime}\right) \mathcal{D}_{\lambda_{j} 0}^{\left(s_{j}\right)}\left(\widehat{\mathbf{q}}_{j}\right),
\end{aligned}
$$

where $\overline{\mathbf{q}}_{j}$ is the momentum of the first particle in the final state in the $\mathrm{IRF}_{j}$, and $\overline{\mathbf{q}}_{k}^{\prime}$ is the momentum of the first particle in the initial state in the $\mathrm{IRF}_{k}^{\prime}$. Since $\mathbf{p}_{k}^{\prime \prime}=\mathbf{p}_{k}^{\prime}$ and $\mathbf{p}_{j}^{\prime \prime}=\mathbf{p}_{j}$, then the azimuthal angles are identical, $\gamma_{k}^{\prime}=\gamma_{j}$. The helicity angles of the first particle in the opposite frames are defined as $\bar{\chi}_{j}$ and $\bar{\chi}_{k}^{\prime}$, cf. Fig. C.3. However, we can easily identify that $\chi_{k}^{\prime}=\bar{\chi}_{k}^{\prime}$ and $\chi_{j}=\overline{\chi_{k}^{\prime}}$ since the intermediate spectator is aligned for the OPE and the IRFs merely differ by the rotation about $z$.

Combining all of this together yields the PWIS unitarity relations,

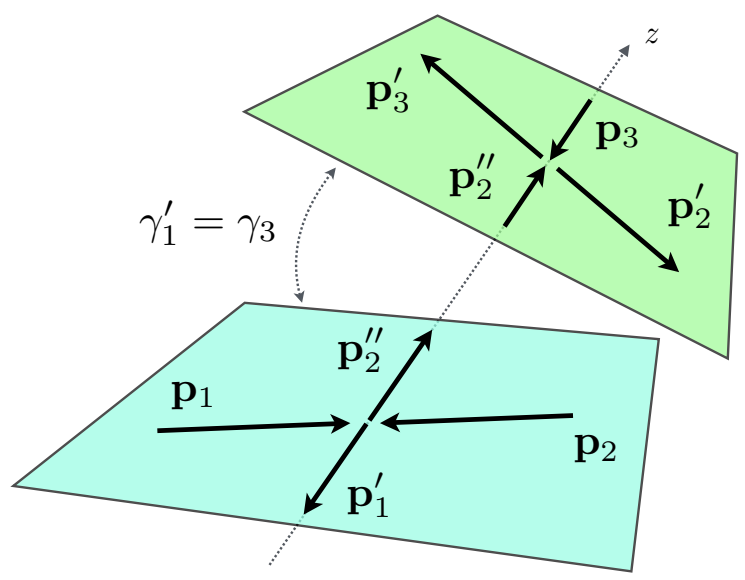

Figure C.3: Relation between the initial and final IRF planes for the OPE amplitude. 


$$
\begin{aligned}
& \operatorname{Im} \mathcal{A}_{\ell_{k}^{\prime} s_{k}^{\prime} ; \ell_{j} s_{j}}^{J}\left(\sigma_{k}^{\prime}, s, \sigma_{j}\right) \\
& =\frac{1}{\pi\left(32 \pi^{2}\right)^{2}} \sum_{n} \sum_{\ell_{n}^{\prime \prime}, s_{n}^{\prime \prime}} \int_{\sigma_{n}^{(\mathrm{th})}}^{\left(\sqrt{s}-m_{n}\right)^{2}} d \sigma_{n}^{\prime \prime} \frac{\left|\mathbf{q}_{n}^{\prime \prime}\right|\left|\mathbf{p}_{n}^{\prime \prime \star}\right|}{\sqrt{\sigma_{n}^{\prime \prime} \sqrt{s}}} \\
& \times \mathcal{A}_{\ell_{n}^{\prime \prime} s_{n}^{\prime \prime} ; \ell_{k}^{\prime} s_{k}^{\prime}}^{J *}\left(\sigma_{n}^{\prime \prime}, s, \sigma_{k}^{\prime}\right) \mathcal{A}_{\ell_{n}^{\prime \prime} s_{n}^{\prime \prime} ; \ell_{j} s_{j}}^{J}\left(\sigma_{n}^{\prime \prime}, s, \sigma_{j}\right) \Theta\left(s-s_{\mathrm{th}}\right) \\
& +\frac{1}{2 \pi s\left(32 \pi^{2}\right)^{2}} \sum_{\substack{n, r \\
n \neq r}} \sum_{\ell_{n}^{\prime \prime}, s_{n}^{\prime \prime}} \sum_{\ell_{r}^{\prime \prime}, s_{r}^{\prime \prime}} \int_{\sigma_{n}^{(\text {th })}}^{\left(\sqrt{s}-m_{n}\right)^{2}} d \sigma_{n}^{\prime \prime} \int_{\sigma_{r}^{(-)}\left(\sigma_{n}^{\prime \prime}\right)}^{\sigma_{r}^{(+)}\left(\sigma_{n}^{\prime \prime}\right)} d \sigma_{r}^{\prime \prime} \\
& \times \mathcal{C}_{\ell_{n}^{\prime \prime} s_{n}^{\prime \prime} ; \ell_{r}^{\prime \prime} s_{r}^{\prime \prime}}^{J}\left(\sigma_{n}^{\prime \prime}, s, \sigma_{r}^{\prime \prime}\right) \mathcal{A}_{\ell_{r}^{\prime \prime} s_{r}^{\prime \prime} ; \ell_{k}^{\prime} s_{k}^{\prime}}^{J *}\left(\sigma_{r}^{\prime \prime}, s, \sigma_{k}^{\prime}\right) \mathcal{A}_{\ell_{n}^{\prime \prime} s_{n}^{\prime \prime} ; \ell_{j} s_{j}}^{J}\left(\sigma_{n}^{\prime \prime}, s, \sigma_{j}\right) \Theta\left(s-s_{\mathrm{th}}\right) \\
& +\rho_{2}\left(\sigma_{k}^{\prime}\right) f_{s_{k}^{\prime}}^{*}\left(\sigma_{k}^{\prime}\right) \mathcal{A}_{\ell_{k}^{\prime} s_{k}^{\prime} ; \ell_{j} s_{j}}^{J}\left(\sigma_{k}^{\prime}, s, \sigma_{j}\right) \\
& +\rho_{2}\left(\sigma_{j}\right) \mathcal{A}_{\ell_{k}^{\prime} s_{k}^{\prime} ; \ell_{j} s_{j}}^{J *}\left(\sigma_{k}^{\prime}, s, \sigma_{j}\right) f_{s_{j}}\left(\sigma_{j}\right) \\
& +\frac{1}{64 \pi^{2} \sqrt{s}} \frac{1}{\left|\mathbf{p}_{k}^{\prime \star}\right|} \sum_{\substack{r \\
r \neq k}} \sum_{\ell_{r}^{\prime \prime}, s_{r}^{\prime \prime}} \int_{\sigma_{r}^{(\mathrm{th})}}^{\left(\sqrt{s}-m_{r}\right)^{2}} d \sigma_{r}^{\prime \prime} \mathcal{C}_{\ell_{k}^{\prime} s_{k}^{\prime} ; \ell_{r}^{\prime \prime} s_{r}^{\prime \prime}}^{J}\left(\sigma_{k}^{\prime}, s, \sigma_{r}^{\prime \prime}\right) \\
& \times f_{s_{k}^{\prime}}^{*}\left(\sigma_{k}^{\prime}\right) \mathcal{A}_{\ell_{r}^{\prime \prime} s_{r}^{\prime \prime} ; \ell_{j} s_{j}}^{J}\left(\sigma_{r}^{\prime \prime}, s, \sigma_{j}\right) \Theta\left(\sigma_{k}^{\prime}-\sigma_{k}^{(\mathrm{th})}\right) \\
& +\frac{1}{64 \pi^{2} \sqrt{s}} \frac{1}{\left|\mathbf{p}_{j}^{\star}\right|} \sum_{\substack{n \neq j \\
n \neq j}} \sum_{\ell_{n}^{\prime \prime}, s_{n}^{\prime \prime}} \int_{\sigma_{n}^{(\mathrm{th})}}^{\left(\sqrt{s}-m_{n}\right)^{2}} d \sigma_{n}^{\prime \prime} \mathcal{C}_{\ell_{n}^{\prime \prime} s_{n}^{\prime \prime} ; \ell_{j} s_{j}}^{J}\left(\sigma_{n}^{\prime \prime}, s, \sigma_{j}\right) \\
& \times f_{s_{j}}\left(\sigma_{j}\right) \mathcal{A}_{\ell_{k}^{\prime} s_{k}^{\prime} ; \ell_{n}^{\prime \prime} s_{n}^{\prime \prime}}^{J}\left(\sigma_{k}^{\prime}, s, \sigma_{n}^{\prime \prime}\right) \Theta\left(\sigma_{j}-\sigma_{j}^{(\text {th })}\right) \\
& +\frac{\pi}{2\left|\mathbf{p}_{j}^{\star}\right|\left|\mathbf{p}_{k}^{\prime \star}\right|} f_{s_{k}^{\prime}}^{*}\left(\sigma_{k}^{\prime}\right) f_{s_{j}}\left(\sigma_{j}\right) \mathcal{C}_{\ell_{k}^{\prime} s_{k}^{\prime} ; \ell_{j} s_{j}}^{J}\left(\sigma_{k}^{\prime}, s, \sigma_{j}\right)\left(1-\delta_{j k}\right) \Theta\left(1-\left|z_{k j}\right|^{2}\right),
\end{aligned}
$$

where the recoupling coefficients are defined in Eq. 4.19. Notice that the first, third, and fourth term involve direct channel exchanges in the intermediate state, while the others involve rescattering between cross channels.

Finally, we introduce the amputated amplitude $\widetilde{\mathcal{A}}_{\ell_{k}^{\prime} s_{k}^{\prime} ; \ell_{j} s_{j}}^{J}$, defined in Eq. 4.18. The amputation eliminates the unitarity cut from the $\mathbf{2} \rightarrow \mathbf{2}$ amplitude in the two particle subsystem in the third and fourth term of Eq. (C.18). Taking the imaginary part of Eq. 4.18,

$$
\begin{aligned}
\operatorname{Im}\left[f_{s_{k}^{\prime}}\left(\sigma_{k}^{\prime}\right) \widetilde{\mathcal{A}}_{\ell_{k}^{\prime} s_{k}^{\prime} ; \ell_{j} s_{j}}\left(\sigma_{k}^{\prime}, s, \sigma_{j}\right) f_{s_{j}}\left(\sigma_{j}\right)\right]= & \operatorname{Im}\left[f_{s_{k}^{\prime}}\left(\sigma_{k}^{\prime}\right)\right] \widetilde{\mathcal{A}}_{\ell_{k}^{\prime} s_{k}^{\prime} ; \ell_{j} s_{j}}\left(\sigma_{k}^{\prime}, s, \sigma_{j}\right) f_{s_{j}}\left(\sigma_{j}\right) \\
& +f_{s_{k}^{\prime}}^{*}\left(\sigma_{k}^{\prime}\right) \operatorname{Im}\left[\widetilde{\mathcal{A}}_{\ell_{k}^{\prime} s_{k}^{\prime} ; \ell_{j} s_{j}}\left(\sigma_{k}^{\prime}, s, \sigma_{j}\right)\right] f_{s_{j}}\left(\sigma_{j}\right) \\
& +f_{s_{k}^{\prime}}^{*}\left(\sigma_{k}^{\prime}\right) \widetilde{\mathcal{A}}_{\ell_{k}^{\prime} s_{k}^{\prime} ; \ell_{j} s_{j}}^{*}\left(\sigma_{k}^{\prime}, s, \sigma_{j}\right) \operatorname{Im}\left[f_{s_{j}}\left(\sigma_{j}\right)\right] .
\end{aligned}
$$


The amputation removes the contribution from the isobar amplitude unitarity cut using Eq. (4.9), leaving only rescattering corrections to the isobar shape. We then arrive at the amputated PWIS unitarity relations Eq. 4.19. 


\section{Appendix D}

\section{THE $B$-MATRIX AND UNITARITY}

In this appendix, we demonstrate that the $B$-matrix parameterization satisfies the unitarity relations Eq. 4.19$)$, specifically for $\widetilde{\mathcal{A}}_{13}$. Recall that the $B$-matrix parameterization for $\widetilde{\mathcal{A}}_{13}$ is

$$
\widetilde{\mathcal{A}}_{13}(s)=\widetilde{\mathcal{B}}_{13}(s)+\widetilde{\mathcal{B}}_{13}(s) \tau_{3}(s) \widetilde{\mathcal{A}}_{33}(s)
$$

which has an imaginary part

$$
\begin{aligned}
\operatorname{Im} \widetilde{\mathcal{A}}_{13}(s) & =\operatorname{Im} \widetilde{\mathcal{B}}_{13}(s) \\
& +\operatorname{Im} \widetilde{\mathcal{B}}_{13}(s) \tau_{3}(s) \widetilde{\mathcal{A}}_{33}(s) \\
& +\widetilde{\mathcal{B}}_{13}^{*}(s) \operatorname{Im} \tau_{3}(s) \widetilde{\mathcal{A}}_{33}(s) \\
& +\widetilde{\mathcal{B}}_{13}^{*}(s) \tau_{3}^{*}(s) \operatorname{Im} \widetilde{\mathcal{A}}_{33}(s) .
\end{aligned}
$$

From Eqs. 5.4 and 4.9,

$$
\operatorname{Im} \tau_{n}\left(s, \sigma_{n}\right)=\rho_{3}\left(s, \sigma_{n}\right) \rho_{2}\left(\sigma_{n}\right)\left|f_{s_{n}}\left(\sigma_{n}\right)\right|^{2},
$$

and since $\widetilde{\mathcal{R}}_{k j}$ is real, $\operatorname{Im} \widetilde{\mathcal{B}}_{k j}=\operatorname{Im} \widetilde{\mathcal{E}}_{k j}$, which is known from projecting Eq. (5.6) into partial waves.

The imaginary part of $\widetilde{\mathcal{A}}_{33}$ is found by using Eq. (5.12b),

$$
\begin{aligned}
\operatorname{Im} \widetilde{\mathcal{A}}_{33}(s) & =\operatorname{Im}\left[\mathbb{1}-\mathcal{K}_{33}(s) \tau_{3}(s)\right]^{-1} \mathcal{K}_{33}(s) \\
& +\left[\mathbb{1}-\mathcal{K}_{33}^{*}(s) \tau_{3}^{*}(s)\right]^{-1} \operatorname{Im} \mathcal{K}_{33}(s)
\end{aligned}
$$

where the kernel $\mathcal{K}_{33}(s)=\widetilde{\mathcal{B}}_{31}(s) \tau_{1}(s) \widetilde{\mathcal{B}}_{13}(s)$. The imaginary part of $\left[\mathbb{1}-\mathcal{K}_{33}(s) \tau_{3}(s)\right]^{-1}$ is found 
by the identity $\operatorname{Im}\left[A^{-1} A\right]=\operatorname{Im} A^{-1} A+A^{*-1} \operatorname{Im} A=0$, giving

$$
\begin{aligned}
\operatorname{Im}\left[\mathbb{1}-\mathcal{K}_{33}(s) \tau_{3}(s)\right]^{-1} & =\left[\mathbb{1}-\mathcal{K}_{33}^{*}(s) \tau_{3}^{*}(s)\right]^{-1} \\
& \times \operatorname{Im}\left[\mathcal{K}_{33}(s) \tau_{3}(s)\right] \\
& \times\left[\mathbb{1}-\mathcal{K}_{33}(s) \tau_{3}(s)\right]^{-1},
\end{aligned}
$$

with $\operatorname{Im}\left[\mathcal{K}_{33}(s) \tau_{3}(s)\right]=\operatorname{Im} \mathcal{K}_{33}(s) \tau_{3}(s)+\mathcal{K}_{33}^{*}(s) \operatorname{Im} \tau_{3}(s)$. Combining Eqs. (D.2), (D.4), and (D.5) give

$$
\begin{aligned}
\operatorname{Im} \widetilde{\mathcal{A}}_{13}(s) & =\operatorname{Im} \widetilde{\mathcal{B}}_{13}(s) \\
& +\operatorname{Im} \widetilde{\mathcal{B}}_{13}(s) \tau_{3}(s) \widetilde{\mathcal{A}}_{33}(s) \\
& +\widetilde{\mathcal{B}}_{13}^{*}(s) \operatorname{Im} \tau_{3}(s) \widetilde{\mathcal{A}}_{33}(s) \\
& +\widetilde{\mathcal{B}}_{13}^{*}(s) \tau_{3}^{*}(s)\left[\mathbb{1}-\mathcal{K}_{33}^{*}(s) \tau_{3}^{*}(s)\right]^{-1} \operatorname{Im} \mathcal{K}_{33}(s) \tau_{3}(s)\left[\mathbb{1}-\mathcal{K}_{33}(s) \tau_{3}(s)\right]^{-1} \mathcal{K}_{33}(s) \\
& +\widetilde{\mathcal{B}}_{13}^{*}(s) \tau_{3}^{*}(s)\left[\mathbb{1}-\mathcal{K}_{33}^{*}(s) \tau_{3}^{*}(s)\right]^{-1} \mathcal{K}_{33}^{*}(s) \operatorname{Im} \tau_{3}(s)\left[\mathbb{1}-\mathcal{K}_{33}(s) \tau_{3}(s)\right]^{-1} \mathcal{K}_{33}(s) \\
& +\widetilde{\mathcal{B}}_{13}^{*}(s) \tau_{3}^{*}\left[\mathbb{1}-\mathcal{K}_{33}^{*}(s) \tau_{3}^{*}(s)\right]^{-1} \operatorname{Im} \mathcal{K}_{33}(s) .
\end{aligned}
$$

The imaginary part of the kernel is

$$
\begin{aligned}
\operatorname{Im} \mathcal{K}_{33}(s) & =\operatorname{Im} \widetilde{\mathcal{B}}_{31}(s) \tau_{1}(s) \widetilde{\mathcal{B}}_{13}(s) \\
& +\widetilde{\mathcal{B}}_{31}^{*}(s) \operatorname{Im} \tau_{1}(s) \widetilde{\mathcal{B}}_{13}(s) \\
& +\widetilde{\mathcal{B}}_{31}^{*}(s) \tau_{1}^{*}(s) \operatorname{Im} \widetilde{\mathcal{B}}_{13}(s) .
\end{aligned}
$$


We use Eq. (5.15) to shift the last three lines of Eq. (D.6) in terms $\mathcal{K}_{11}=\widetilde{\mathcal{B}}_{13}(s) \tau_{3}(s) \widetilde{\mathcal{B}}_{31}(s)$,

$$
\begin{aligned}
\operatorname{Im} \widetilde{\mathcal{A}}_{13}(s) & =\operatorname{Im} \widetilde{\mathcal{B}}_{13}(s) \\
& +\operatorname{Im} \widetilde{\mathcal{B}}_{13}(s) \tau_{3}(s) \widetilde{\mathcal{A}}_{33}(s) \\
& +\widetilde{\mathcal{B}}_{13}^{*}(s) \operatorname{Im} \tau_{3}(s) \widetilde{\mathcal{A}}_{33}(s) \\
& +\left[\mathbb{1}-\mathcal{K}_{11}^{*}(s) \tau_{1}^{*}(s)\right]^{-1} \widetilde{\mathcal{B}}_{13}^{*}(s) \tau_{3}^{*} \operatorname{Im} \widetilde{\mathcal{B}}_{31}(s) \tau_{1}(s) \widetilde{\mathcal{B}}_{13}(s) \tau_{3}(s)\left[\mathbb{1}-\mathcal{K}_{33}(s) \tau_{3}(s)\right]^{-1} \mathcal{K}_{33}(s) \\
& +\left[\mathbb{1}-\mathcal{K}_{11}^{*}(s) \tau_{1}^{*}(s)\right]^{-1} \mathcal{K}_{11}^{*}(s) \operatorname{Im} \tau_{1}(s) \widetilde{\mathcal{B}}_{13}(s) \tau_{3}(s)\left[\mathbb{1}-\mathcal{K}_{33}(s) \tau_{3}(s)\right]^{-1} \mathcal{K}_{33}(s) \\
& +\left[\mathbb{1}-\mathcal{K}_{11}^{*}(s) \tau_{1}^{*}(s)\right]^{-1} \mathcal{K}_{11}^{*}(s) \tau_{1}^{*}(s) \operatorname{Im} \widetilde{\mathcal{B}}_{13}(s) \tau_{3}(s)\left[\mathbb{1}-\mathcal{K}_{33}(s) \tau_{3}(s)\right]^{-1} \mathcal{K}_{33}(s) \\
& +\widetilde{\mathcal{B}}_{13}^{*}(s) \tau_{3}^{*}(s)\left[\mathbb{1}-\mathcal{K}_{33}^{*}(s) \tau_{3}^{*}(s)\right]^{-1} \mathcal{K}_{33}^{*}(s) \operatorname{Im} \tau_{3}(s)\left[\mathbb{1}-\mathcal{K}_{33}(s) \tau_{3}(s)\right]^{-1} \mathcal{K}_{33}(s) \\
& +\left[\mathbb{1}-\mathcal{K}_{11}^{*}(s) \tau_{1}^{*}(s)\right]^{-1} \widetilde{\mathcal{B}}_{13}^{*}(s) \tau_{3}^{*}(s) \operatorname{Im} \widetilde{\mathcal{B}}_{31}(s) \tau_{1}(s) \widetilde{\mathcal{B}}_{13}(s) \\
& +\left[\mathbb{1}-\mathcal{K}_{11}^{*}(s) \tau_{1}^{*}(s)\right]^{-1} \mathcal{K}_{11}^{*}(s) \operatorname{Im} \tau_{1}(s) \widetilde{\mathcal{B}}_{13}(s) \\
& +\left[\mathbb{1}-\mathcal{K}_{11}^{*}(s) \tau_{1}^{*}(s)\right]^{-1} \mathcal{K}_{11}^{*}(s) \tau_{1}^{*}(s) \operatorname{Im} \widetilde{\mathcal{B}}_{13}(s) .
\end{aligned}
$$

Grouping common terms in $\operatorname{Im} \tau_{n}$ and $\operatorname{Im} \widetilde{\mathcal{B}}_{k j}$, and identifying the forms of the amplitudes from Sec. ??, yields

$$
\begin{aligned}
\operatorname{Im} \widetilde{\mathcal{A}}_{13}(s) & =\operatorname{Im} \widetilde{\mathcal{B}}_{13}(s) \\
& +\operatorname{Im} \widetilde{\mathcal{B}}_{13}(s) \tau_{3}(s) \widetilde{\mathcal{A}}_{33}(s) \\
& +\widetilde{\mathcal{A}}_{11}^{*}(s) \tau_{1}^{*}(s) \operatorname{Im} \widetilde{\mathcal{B}}_{13}(s) \\
& +\widetilde{\mathcal{A}}_{13}^{*}(s) \operatorname{Im} \tau_{3}(s) \widetilde{\mathcal{A}}_{33}(s) \\
& +\widetilde{\mathcal{A}}_{11}^{*}(s) \operatorname{Im} \tau_{1}(s) \widetilde{\mathcal{A}}_{13}(s) \\
& +\widetilde{\mathcal{A}}_{11}^{*}(s) \tau_{1}^{*}(s) \operatorname{Im} \widetilde{\mathcal{B}}_{13}(s) \tau_{3}(s) \widetilde{\mathcal{A}}_{33}(s) \\
& +\widetilde{\mathcal{A}}_{13}^{*}(s) \tau_{3}^{*}(s) \operatorname{Im} \widetilde{\mathcal{B}}_{31}(s) \tau_{1}(s) \widetilde{\mathcal{A}}_{13}(s) .
\end{aligned}
$$

Substituting for the imaginary parts of $\tau_{n}$ and $\widetilde{\mathcal{B}}_{k j}$ gives the PWIS unitarity relation for $\widetilde{\mathcal{A}}_{13}$. The unitarity relations for the other amplitudes can be found in a similar manner. 


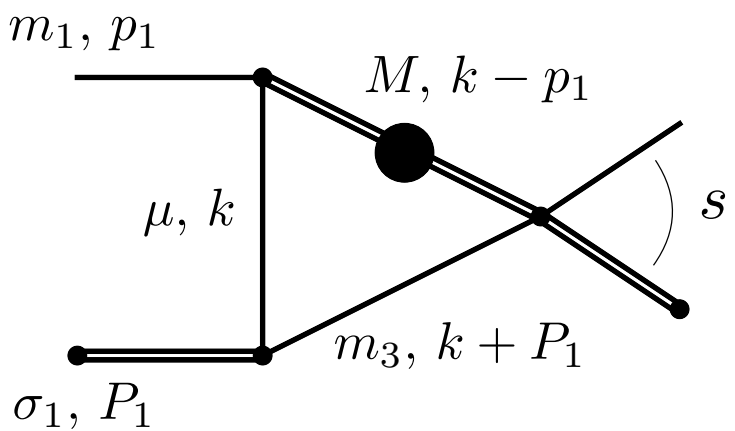

Figure E.1: The triangle diagram with loop momentum labels.

\section{Appendix E}

\section{THE FEYNMAN TRIANGLE DIAGRAM}

For reference, we state the basic formulae for computing the Feynman triangle diagram, cf. Ref. [74. The perturbative Feynman diagram has the form

$$
\mathcal{T}_{F}(s)=i \int \frac{d^{4} k}{(2 \pi)^{4}} \frac{1}{D_{1} D_{2} D_{3}},
$$

shown in Fig. E.1, where the denominator is the product of internal propagators,

$$
\begin{aligned}
D_{1} D_{2} D_{3} & =\left[k^{2}-\mu_{j k}^{2}+i \epsilon\right] \\
& \times\left[\left(k+P_{1}\right)^{2}-m_{3}^{2}+i \epsilon\right] \\
& \times\left[\left(k-p_{1}\right)^{2}-M^{2}+i \epsilon\right] .
\end{aligned}
$$

Using the Feynman parameterization and standard loop integration techniques, the Feynman diagram has the form

$$
\mathcal{T}_{F}(s)=\frac{1}{16 \pi^{2}} \int_{0}^{1} d \alpha_{1} \int_{0}^{1-\alpha_{1}} d \alpha_{2} F\left(s ; \alpha_{1}, \alpha_{2}\right)
$$


where

$$
\begin{aligned}
F^{-1}\left(s ; \alpha_{1}, \alpha_{2}\right) & =M^{2} \alpha_{1}+m_{3}^{2} \alpha_{2}+\mu^{2}\left(1-\alpha_{1}-\alpha_{2}\right) \\
& +m_{1}^{2} \alpha_{1}\left(\alpha_{1}-1\right)+\sigma_{1} \alpha_{2}\left(\alpha_{2}-1\right) \\
& -\left(s-\sigma_{1}-m_{1}^{2}\right) \alpha_{1} \alpha_{2}-i \epsilon .
\end{aligned}
$$

The remaining integrals over the Feynman parameters can be computed either numerically, or by analytically performing the integral over $\alpha_{2}$, then numerically computing the remaining integral over $\alpha_{1}$.

Alternatively, the Feynman triangle can be written with a dispersive representation in $s$ using the Cutkosky rules 74 ,

$$
\mathcal{T}_{F}(s)=\int_{\Gamma_{T}} d s^{\prime} \frac{\rho_{3}\left(s^{\prime}, M^{2}\right) \widetilde{\mathcal{E}}_{13}\left(M^{2}, s^{\prime}, \sigma_{1}\right)}{s^{\prime}-s-i \epsilon},
$$

where $\Gamma_{T}$ is the path from the threshold $\left(M+m_{3}\right)^{2}$ to $\infty, \rho_{3}\left(s, M^{2}\right)$ is given by Eq. (5.5), $\widetilde{\mathcal{E}}_{13}$ is given by Eq. (5.17), and the $S$-wave amplitudes are normalized according to Eq. 4.15). The phase space contributes branch point singularities from the threshold and pseudothreshold, $\left(M \pm m_{3}\right)^{2}$, and a pole at $s=0$. The OPE has branch points $s=s_{31}^{( \pm)}$near the integration region. Following the discussion in Section 5.2.1, the OPE branch points give us the following scenarios:

(a) $M^{2} \geq \sigma_{3}^{(b)}$. The RPE branch points pinch the integration region which starts at $s=\left(M+m_{3}\right)^{2}$. Figure E.2(a) shows the integrand branch cuts and the dispersive contour. The RPE branch point $s_{31}^{(-)}$lie in the unphysical sheet close to threshold, causing the known as the triangle singularity $86,94,95,98$. The triangle singularity produces an extra threshold in the physical region above the threshold $s=\left(M+m_{3}\right)^{2}$, and is associated with real particle exchange in the intermediate state. The location of the triangle singularity occurs at

$$
\begin{aligned}
s_{\mathrm{tri}} & =\frac{1}{2 m_{2}^{2}}\left[\left(m_{3}^{2}-\sigma_{1}\right)\left(m_{1}^{2}-M^{2}\right)-m_{2}^{4}\right. \\
& +m_{2}^{2}\left(m_{3}^{2}+m_{1}^{2}+\sigma_{1}+M^{2}\right) \\
& \left. \pm \lambda^{1 / 2}\left(m_{2}^{2}, m_{3}^{2}, \sigma_{1}\right) \lambda^{1 / 2}\left(m_{2}^{2}, m_{1}^{2}, M^{2}\right)\right] .
\end{aligned}
$$



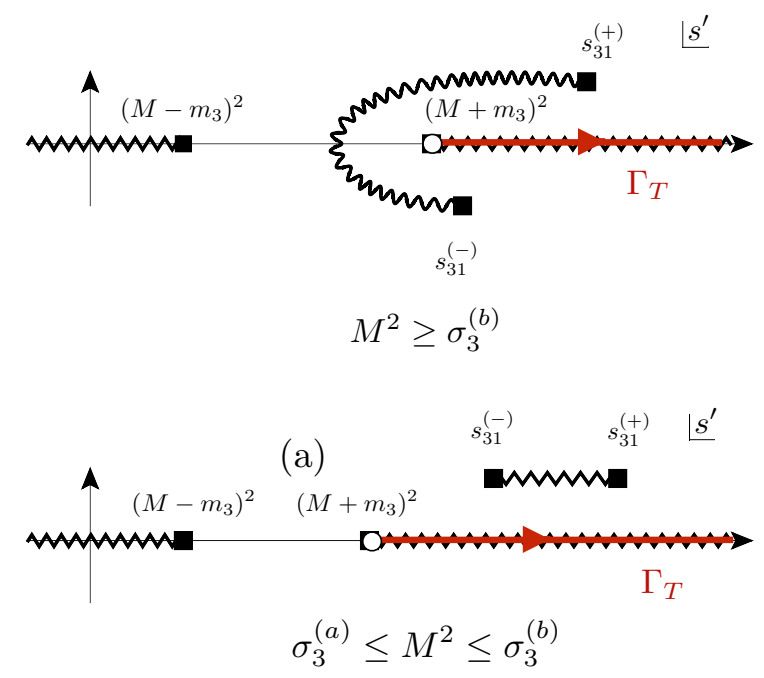

(b)

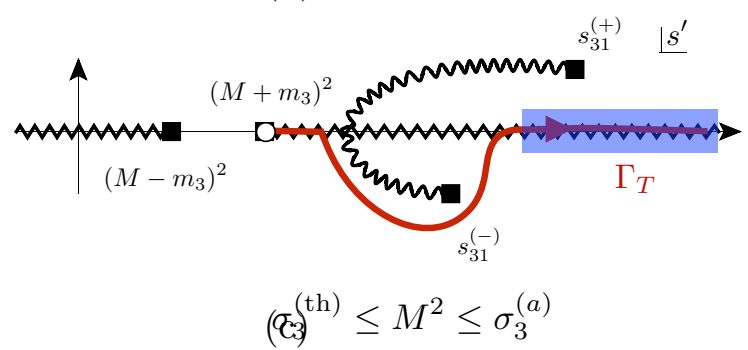

Figure E.2: Contours for dispersive triangle Eq. (5.29) shown in red, and the integrand cuts. The three cases are (a) $M^{2} \geq \sigma_{3}^{(b)}$, (b) $\sigma_{3}^{(a)} \leq M^{2} \leq \sigma_{3}^{(b)}$, and (c) $\sigma_{3}^{(\text {th })} \leq M^{2} \leq \sigma_{3}^{(a)}$. Case (a) corresponds to the usual triangle singularity, which occurs since the OPE branch points pinch the integration region. Case (c) happens when the initial state of the OPE has a higher threshold then the intermediate state. The blue region indicates the physical region from the initial threshold. Note that a triangle singularity does not occur in this case and the integration is not pinched.

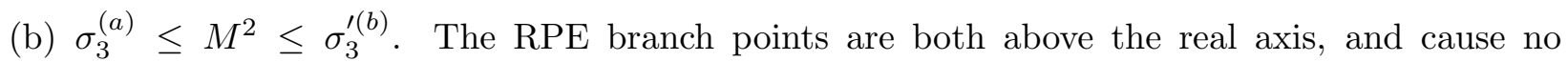
additional singular behavior. Figure E.2(b) illustrates this case.

(c) $\sigma_{3}^{(\text {th })} \leq M^{2} \leq \sigma_{3}^{(a)}$. The RPE branch points are again on opposite sides of the real axis. However, the integration region begins below the location where the RPE cut crosses the real axis. This is due to the fact that the initial state has a higher threshold then the intermediate 
state, so the physical region is above the RPE crossing location. This is illustrated in Fig. E.2 where the blue region indicates the physical region starting at the initial state threshold, and the integration contour is a path around the RPE branch point $s_{31}^{(-)}$. No singularity occurs in this region as the RPE branch points do not pinch the integration region.

Notice that in contrast to the $B$-matrix triangle, Eq. (5.27), the dispersive triangle moves all the singularities from the phase space and OPE to the unphysical sheet. Thus, the only singularity present on the physical sheet is the unitarity cut starting at $s=\left(M+m_{3}\right)^{2}$. 


\title{
Curriculum Vitae
}

\author{
Andrew W. Jackura
}

andrew.jackura@gmail.com

\section{Education}

2013-2019 Ph.D. - Indiana University - Physics

2013-2015 M.S. - Indiana University - Physics

2011-2013 B.S. - Purdue University Calumet - Physics

2007-2011 B.S. - Purdue University Calumet - Mechanical Engineering

\section{Publications}

\section{Papers}

1. A. Rodas et al. [JPAC Collaboration], "Determination of the pole position of the lightest hybrid meson candidate," Phys. Rev. Lett. 122, no. 4, 042002 (2019)

2. M. Mikhasenko et al. [JPAC Collaboration], "Pole position of the $a_{1}(1260)$ from $\tau$-decay," Phys. Rev. D 98, no. 9, 096021 (2018)

3. A. Jackura et al. [JPAC Collaboration], "Phenomenology of Relativistic $3 \rightarrow 3$ Reaction Amplitudes within the Isobar Approximation," Eur. Phys. J. C 79, no. 1, 56 (2019)

4. J. A. Silva-Castro et al. [JPAC Collaboration], "Regge phenomenology of the $N^{*}$ and $\Delta^{*}$ poles," Phys. Rev. D 99, no. 3, 034003 (2019)

5. V. Mathieu et al. [JPAC Collaboration], "Structure of Pion Photoproduction Amplitudes," Phys. Rev. D 98, no. 1, 014041 (2018)

6. J. Nys et al. [JPAC Collaboration], "Global analysis of charge exchange meson production at high energies," Phys. Rev. D 98, no. 3, 034020 (2018) 
7. A. Pilloni et al. [JPAC Collaboration], "What is the right formalism to search for resonances? II. The pentaquark chain," Eur. Phys. J. C 78, no. 9, 727 (2018)

8. M. Albaladejo et al. [JPAC Collaboration], "Khuri-Treiman equations for $\pi \pi$ scattering," Eur. Phys. J. C 78, no. 7, 574 (2018)

9. V. Mathieu et al. [JPAC Collaboration], "Vector Meson Photoproduction with a Linearly Polarized Beam," Phys. Rev. D 97, no. 9, 094003 (2018)

10. M. Mikhasenko et al. [JPAC Collaboration], "What is the right formalism to search for resonances?," Eur. Phys. J. C 78, no. 3, 229 (2018)

11. J. Nys et al. [JPAC Collaboration], "Features of $\pi \Delta$ Photoproduction at High Energies," Phys. Lett. B 779, 77 (2018)

12. V. Mathieu et al., [JPAC Collaboration], "Analyticity Constraints for Hadron Amplitudes: Going High to Heal Low Energy Issues," EPL 122, no. 4, 41001 (2018)

13. A. Jackura et al. [JPAC and COMPASS Collaborations], "New analysis of $\eta \pi$ tensor resonances measured at the COMPASS experiment," Phys. Lett. B 779, 464 (2018)

14. V. Mathieu, J. Nys, C. Fernández-Ramírez, A. Jackura, M. Mikhasenko, A. Pilloni, A. P. Szczepaniak and G. Fox, "On the $\eta$ and $\eta^{\prime}$ Photoproduction Beam Asymmetry at High Energies," Phys. Lett. B 774, 362 (2017)

15. A. Pilloni et al. [JPAC Collaboration], "Amplitude analysis and the nature of the $Z_{c}$ (3900)," Phys. Lett. B 772, 200 (2017)

16. J. Nys et al. [JPAC Collaboration], "Finite-energy sum rules in eta photoproduction off a nucleon," Phys. Rev. D 95, no. 3, 034014 (2017)

17. A. N. Hiller Blin, C. Fernández-Ramírez, A. Jackura, V. Mathieu, V. I. Mokeev, A. Pilloni and A. P. Szczepaniak, "Studying the $P_{c}(4450)$ resonance in $J / \psi$ photoproduction off protons," Phys. Rev. D 94, no. 3, 034002 (2016)

18. R. A. Briceño et al., "Issues and Opportunities in Exotic Hadrons," Chin. Phys. C 40, no. 4, 042001 (2016) 


\section{Proceedings}

19. A. N. Hiller Blin, C. Fernández-Ramírez, A. Jackura, V. Mathieu, V. I. Mokeev, A. Pilloni and A. P. Szczepaniak, "Studying the $P_{c}(4450)$ resonance in $J / \psi$ photoproduction off protons,"

Few Body Syst. 59, no. 5, 104 (2018)

20. A. Jackura [JPAC and COMPASS Collaborations], "Tensor resonances in $\eta \pi$ using COMPASS data," PoS Hadron 2017, 035 (2018)

21. M. Mikhasenko, A. Jackura, B. Ketzer and A. Szczepaniak, "Unitarity approach to the mass-dependent fit of $3 \pi$ resonance production data from the COMPASS experiment,"

EPJ Web Conf. 137, 05017 (2017)

22. A. Jackura, M. Mikhasenko and A. Szczepaniak, "Amplitude analysis of resonant production in three pions," EPJ Web Conf. 130, 05008 (2016) 